\title{
Vehicle to Electric Vehicle Supply Equipment Smart Grid Communications Interface Research and Testing Report
}

Final Report

Kevin Morrow

Dimitri Hochard Jeff Wishart

James Francfort

September 2011

The Idaho National Laboratory is a U.S. Department of Energy National Laboratory Operated by Battelle Energy Alliance 


\title{
Vehicle to Electric Vehicle Supply Equipment Smart Grid Communications Interface Research and Testing Report
}

\author{
Kevin Morrow \\ Dimitri Hochard \\ Jeffrey Wishart \\ James Francfort
}

September 2011

\section{Idaho National Laboratory \\ Idaho Falls, Idaho 83415}

\author{
Prepared for the \\ U.S. Department of Energy \\ Assistant Secretary for Energy Efficiency and Renewable Energy \\ Under DOE Idaho Operations Office \\ Contract DE-AC07-05ID14517
}




DISCLAIMER
This information was prepared as an account of work sponsored by an
agency of the U.S. Government. Neither the U.S. Government nor any
agency thereof, nor any of their employees, makes any warranty, expressed
or implied, or assumes any legal liability or responsibility for the accuracy,
completeness, or usefulness, of any information, apparatus, product, or
process disclosed, or represents that its use would not infringe privately
owned rights. References herein to any specific commercial product,
process, or service by trade name, trade mark, manufacturer, or otherwise,
does not necessarily constitute or imply its endorsement, recommendation,
or favoring by the U.S. Government or any agency thereof. The views and
opinions of authors expressed herein do not necessarily state or reflect
those of the U.S. Government or any agency thereof.




\section{ABSTRACT}

Plug-in electric vehicles (PEVs), including battery electric, plug-in hybrid electric, and extended range electric vehicles, are under evaluation by the U.S. Department of Energy's Advanced Vehicle Testing Activity (AVTA) and other various stakeholders to better understand their capability and potential petroleum reduction benefits. PEVs could allow users to significantly improve fuel economy over a standard hybrid electric vehicles, and in some cases, depending on daily driving requirements and vehicle design, PEVs may have the ability to eliminate petroleum consumption entirely for daily vehicle trips. The AVTA is working jointly with the Society of Automotive Engineers (SAE) to assist in the further development of standards necessary for the advancement of PEVs.

This report analyzes different methods and available hardware for advanced communications between the electric vehicle supply equipment (EVSE) and the PEV; particularly Power Line Devices and their physical layer. Results of this study are not conclusive, but add to the collective knowledge base in this area to help define further testing that will be necessary for the development of the final recommended SAE communications standard.

The Idaho National Laboratory and the Electric Transportation Applications conduct the AVTA for the United States Department of Energy’s Vehicle Technologies Program. 


\section{CONTENTS}

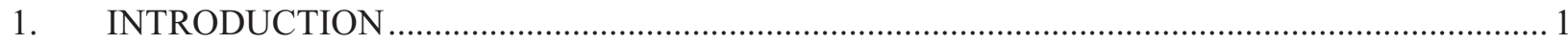

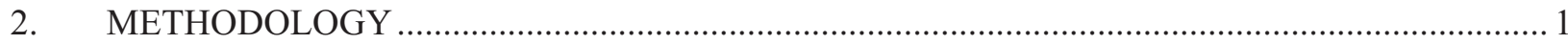

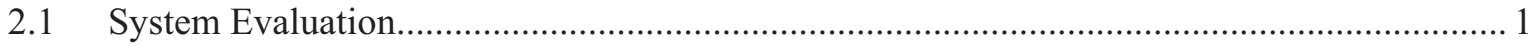

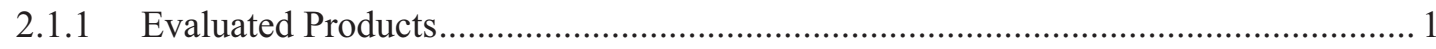

2.2 Functional Testing at the Electric Transportation Applications Engineering Center.............. 3

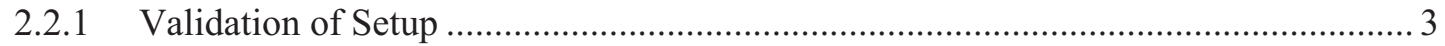

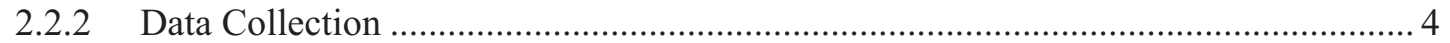

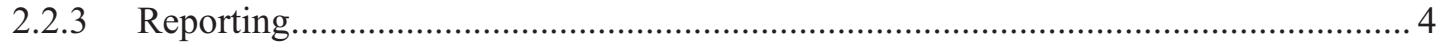

2.3 Electromagnetic Communications Testing at the Electric Transportation

Applications Engineering Center ................................................................................. 4

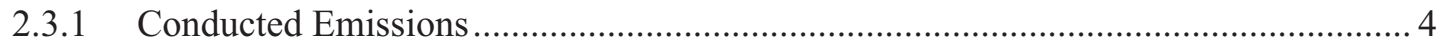

2.4 Electromagnetic Communications Testing at Compliance Testing .................................... 4

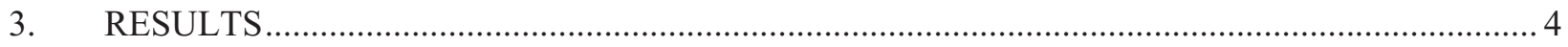

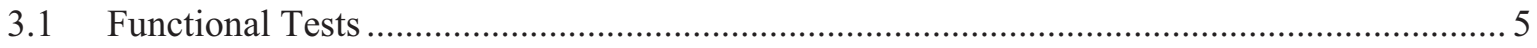

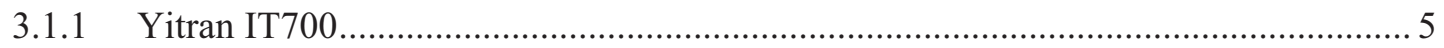

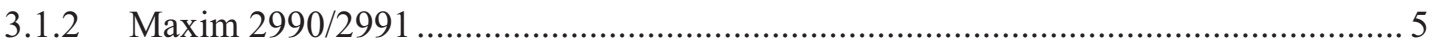

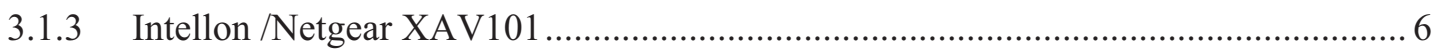

3.2 Baseline Electromagnetic Communications Test Results ................................................... 7

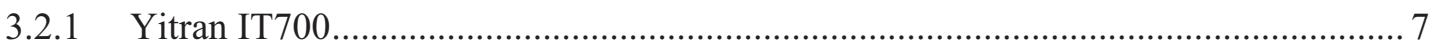

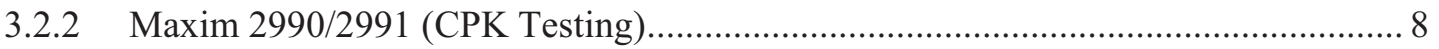

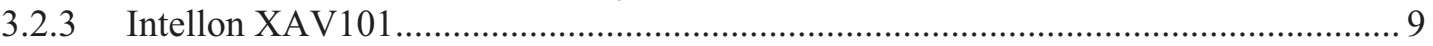

3.3 Formal Electromagnetic Communications Test Results ............................................... 10

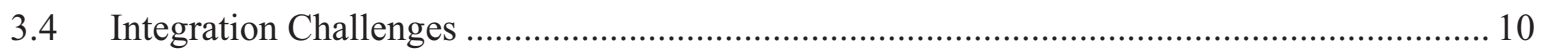

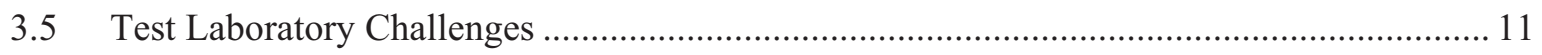

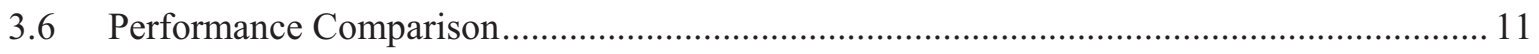

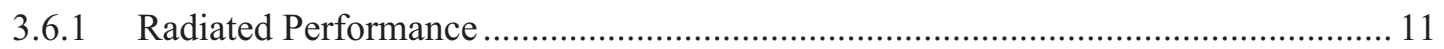

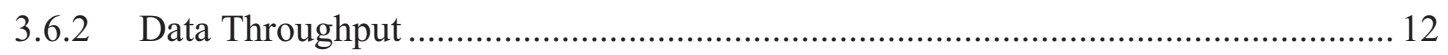

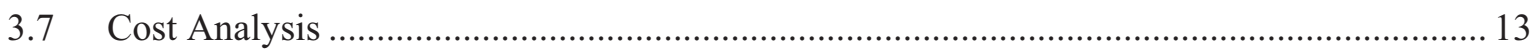

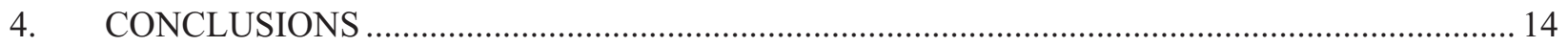




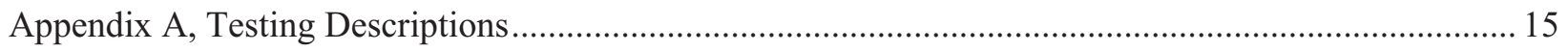

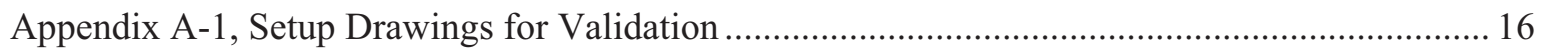

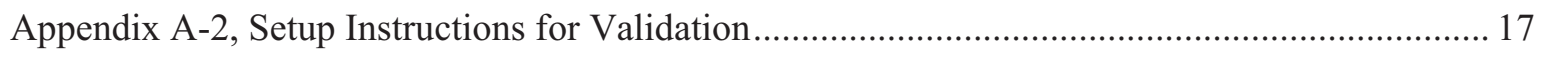

Appendix A-3, Microchip MRF24J40MA Data Sheets ........................................................ 18

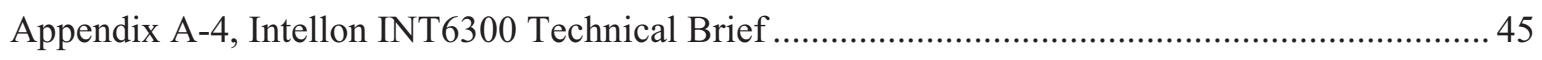

Appendix A-5, Yitran IT700 Technical Brief .......................................................................... 51

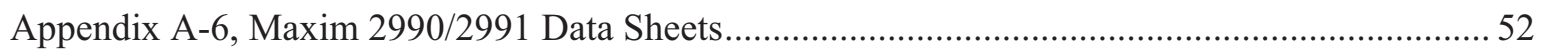

Appendix A-7, Functional Test Setup Drawings.................................................................. 55

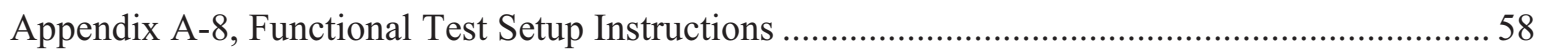

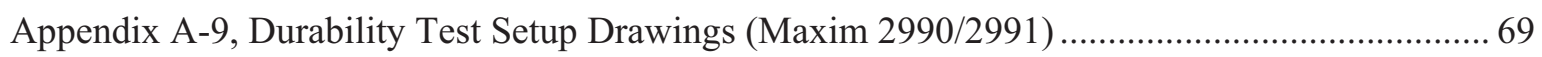

Appendix A-10, Durability Test Setup Instructions (Maxim 2990/2991) .................................... 70

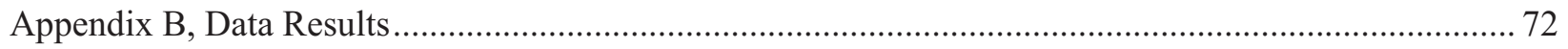

Appendix B-1, Functional Test Results (Yitran IT700) ........................................................... 73

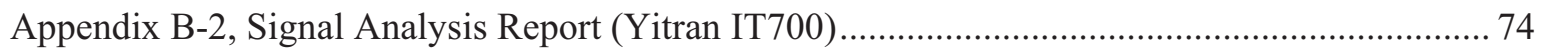

Appendix B-3, Functional Test Results (Maxim 2990/2991) …............................................... 78

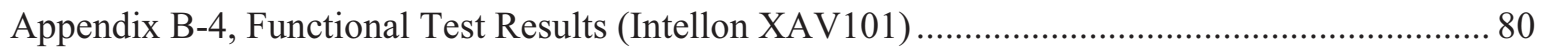

Appendix B-5, Electromagnetic Capability Test Instructions at Electric Transportation

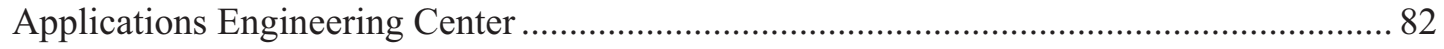

Appendix B-6, Electromagnetic Capability Test Setup Drawings at Electric Transportation Applications Engineering Center .............................................................................. 93

Appendix B-7, Electromagnetic Capability Test Report (CPK - Maxim 2990/2991) ................... 94

Appendix B-8, Electromagnetic Capability Test Report (Compliance Testing) ........................... 97

Appendix B-9, Industrial Application Test Results (Maxim 2990/2991) ................................. 128

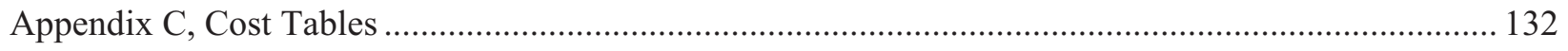

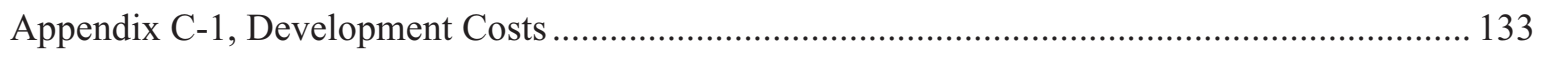

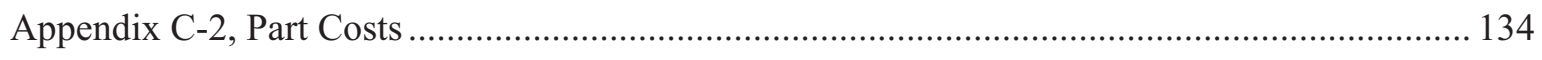

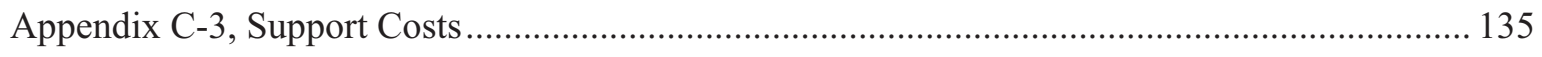




\section{FIGURES}

1. Illustration of the MRF24J40MA microchip from Zigbee ................................................... 2

2. Illustration of the Intellon XAV101 from HomePlug AV ..................................................... 2

3. Illustration of the Yitran IT700 from HomePlug C\&C ........................................................... 3

4. Illustration of the Maxim IC MAX2900/MAX2991 ................................................................

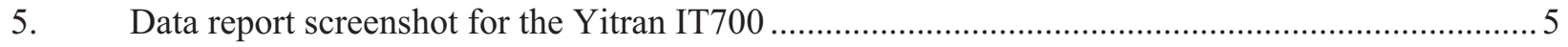

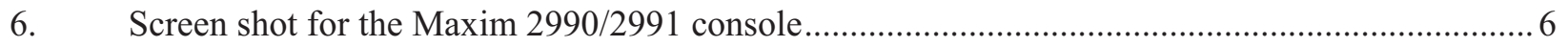

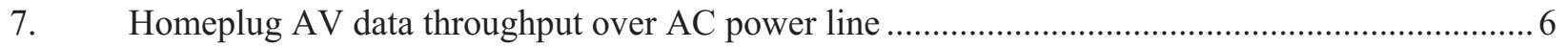

8. Homeplug AV data throughput over DC power line during fast charge .................................... 7

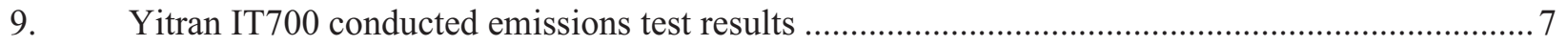

10. Yitran IT700 conducted emissions test results (no transmission) …....................................... 8

11. Radiated emissions test results for the Maxim 2990/2991 device ............................................ 9

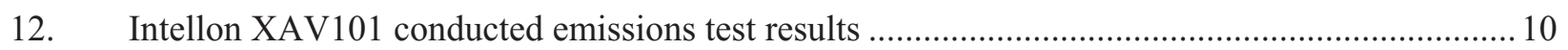

13. Wireless signal traffic results of the Intellon XAV101 product for a separation

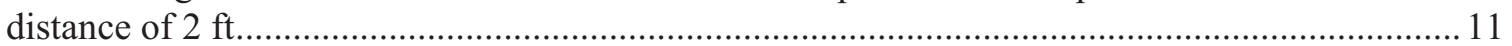

14. Wireless signal traffic results for the Intellon XAV101 product for a separation

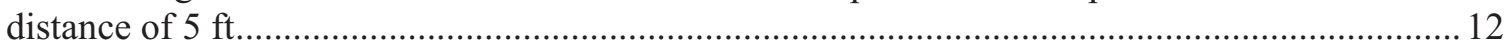

\section{TABLES}

1. Data rate comparison for the three programmable logic controller products 12 


\section{INTRODUCTION}

The U.S Department of Energy's Advanced Vehicle Testing Activity (AVTA) is involved with developing a communication protocol interface between a charger and electric vehicle. The standardization effort consists of a partnership between the U.S Department of Energy (DOE) and the Society of Automotive Engineers (SAE). Committee activities include test reports, evaluation of new and existing communication standards, functional testing of appropriate hardware, coordination between candidate suppliers, specialized testing (e.g., electromagnetic communications testing [EMC]), and a proof of concept validating the interface between the charger and the vehicle. The project will be managed for the committee by the engineering and management team at Electric Transportation Applications (ETA). Project management activities are designed to lead the project using a variety of strategies such as product selection, design of a functional test, selecting an appropriate test center, and supporting all technical aspects of the project in consultation with the SAE committee. Based on a consensus in support of project scope, the following products were selected for evaluation: Yitran IT700, Maxim2990/2991, Intellon XAV101, and Microchip MRF24J40MA. They were selected due to their novelty and potential for meeting the interface requirements listed in Section 2.1 of the scope of work. Other devices using legacy technology (e.g., modulated pilot wire, original SAE J1850 [2-wire], and CAN [2-wire]) were examined in another report and are only referenced in this report. The AVTA activities are managed by the Idaho National Laboratory (INL), and the ETA contract is managed by the National Energy Technology Laboratory (NETL). INL and NETL perform this work for DOE's Vehicle Technologies Program.

\section{METHODOLOGY}

All testing was scheduled and budgeted by ETA engineering and management. Funding was structured in support of completing the system evaluation, functional testing of the candidate system, and EMC testing. Details covering each phase are provided in the following subsections.

\subsection{System Evaluation}

In order to validate a particular candidate solution, a system needed to be designed in a manner specific to the technology and interface requirements of both the electric vehicle charger and the vehicle. Additional criteria, unique to meeting future monitoring needs of the utility, also needed to be considered as stated in Section 2.1.2 of the SAE scope document. This required research involving specific attributes for the candidate technology and interface challenges with the charger and electric vehicle. Collaboration between ETA engineering and SAE committee staff offered possible alternatives in evaluating each candidate system. Based on the collaboration effort, a series of setup drawings and setup instructions for each product under review were created. These items can be found in Appendix A-1 and Appendix A-2, respectively. The evaluation process permitted the team to better examine all possible candidates in a manner that was fair and robust.

\subsubsection{Evaluated Products}

Based on the results of the system evaluation, a series of candidate devices were identified as possible solutions in meeting the needs of the project. With the candidate devices defined, a more rigorous analysis was enabled. Based on consultations with the SAE committee and ETA, a series of detailed functional tests and EMC tests were conducted at the ETA engineering center. Details on the testing are broken down by candidate device in the following subsections.

2.1.1.1 MRF24J40MA - Microchip - (ZigBee). This product is a printed circuit board solution that is Federal Communications Commission (FCC) certified. The product is already in use, requiring no additional testing beyond the functional test. Figure 1 shows the bare device with an arrow showing the antenna hardware. The device must be placed $1 \mathrm{in}$. away from any conducting material, as specified in the datasheet located in Appendix A-3. Multiple tests of similar products have been performed by various 
utility companies. The weaknesses of this solution include having a short range and using a mesh networking device to do point-to-point communication.

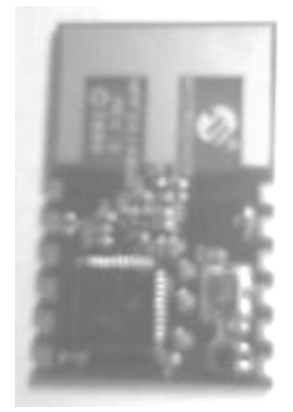

Figure 1. Illustration of the MRF24J40MA microchip from Zigbee.

2.1.1.2 XAV101 - Netgear (Intellon 6300 chipset - HomePlug AV). This product is a system currently in use for delivery of high-speed Internet in consumer electronic products. The device of interest is the chipset, not the device as it is sold in its current form. However, testing the device will illustrate the suitability of the chipset as an integrated solution for high-speed programmable logic controller (PLC) applications in an electrical vehicle charging environment. The XAV101 uses a complex OFDM modulation (using up to 1,155 subcarriers) over the 2 to $28-\mathrm{MHz}$ band. The Netgear XAV101 chip tested acts as a bridge between a network using cat5 cables and a network using a power line as its transport medium. The Netgear device is the only device evaluated that cannot be categorized as an evaluation board. It also is the only device that could not be powered externally, which is a limitation in the sense that it was not possible to isolate the power section from the data bus itself. Figure 2 shows the device in its current form as a Netgear Device. Datasheets detailing specific features of the device can be found in Appendix A-4.

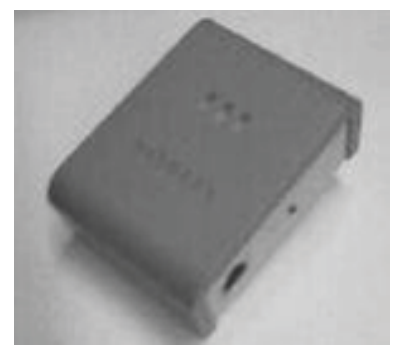

Figure 2. Illustration of the Intellon XAV101 from HomePlug AV.

2.1.1.3 IT700 - Yitran - (HomePlug C\&C). Yitran offers a low-cost, single chip solution. This device uses a patented differential code shift keying PLC operating at a low data rate $(7.5 \mathrm{kbps})$ in the Cenelec OR FCC band. It consists of two modules, one acting as a base station (server) and one acting as a remote station. In its current form, the device pair interfaces with a personal computer hosting the user interface via a USB port. Because this is an evaluation board, additional testing beyond functional is required. Additional testing consists of an EMC validation under FCC and automotive standards. Figure 3 shows a typical Yitran IT700 module. Datasheets for the Yitran IT700 can be found in Appendix A-5. 


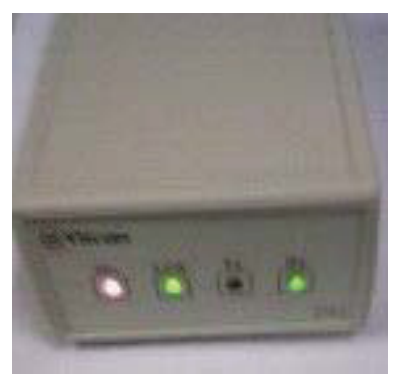

Figure 3. Illustration of the Yitran IT700 from HomePlug C\&C.

2.1.1.4 MAX2990/MAX2991 - Maxim IC. The Maxim modules are a two-chip set solution: one chip acts as the analog front end, while the other supports the orthogonal frequency demodulation process (MAX2990). The orthogonal frequency demodulation is in the Cenelec or FCC band, providing a data throughput of $80 \mathrm{kbps}$ measured. This product was added to the evaluation lineup because Maxim IC had previously done work for smart meters deployed by ERDF (Electricite Reseau Distribution France). Datasheets for the Maxim 2990/2991 devices can be found in Appendix A-6. Figure 4 shows 2990 and 2991 as a stacked pair.

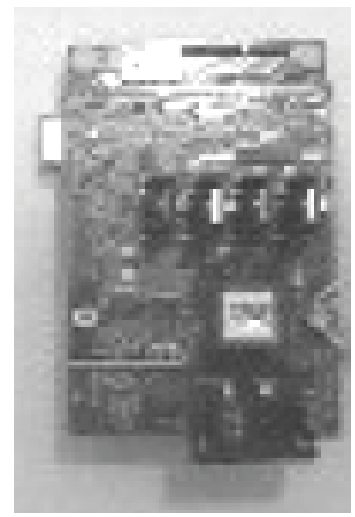

Figure 4. Illustration of the Maxim IC MAX2900/MAX2991.

\subsection{Functional Testing at the Electric Transportation Applications Engineering Center}

\subsubsection{Validation of Setup}

In consultation with SAE and ETA engineering, a validation test plan was established. The goal of the test plan is to secure a method for validating the test setup. Based on summary discussions contained in the monthly progress reports, it was decided the functional test would be designed using a charger-toelectric-vehicle integration model. More specifically, the integration method used by ETA engineering consisted of a Clipper Creek AC Level 2 charger and Mini-E electric vehicle. All four product candidates underwent this phase of initial validation for functional testing. Additionally, testing was done using Energy Technology Engineering Center's industrial chargers and different sizes of battery packs with all three PLC devices validating communication over the direct current (DC) power line when voltage was applied. The modules were tested with different chargers switching at different frequencies to test the effect of the power section on the data throughput of the device signal. Appendix A-7 lists the functional test setup and operation instructions for all four modules under evaluation. Appendix A-8 lists all associated installation drawings for each module undergoing functional testing. Appendix A-9 lists all drawings associated with Maxim2990/2991 in support of the DC charger test. Appendix A-10 lists the 
setup instructions and drawings for the Maxim2990/2991 DC charger test. Similar tests were performed with the Intellon and the Yitran products.

\subsubsection{Data Collection}

Data capture involved using both automated and manual approaches. The automated method was secured by using the device control software provided by the candidate product manufacturer and included using test equipment at the ETA engineering center. Manual data capture was collected in log format, using observation as it pertains to operation of the device.

\subsubsection{Reporting}

This operation organized both manual data and automated data gathered during functional testing. This process also was done for preliminary EMC testing at the ETA engineering center. EMC testing is discussed in Section 2.3.

\subsection{Electromagnetic Communications Testing at the Electric Transportation Applications Engineering Center}

Because of the novelty of PLC communication technology, a baseline average was needed to properly examine the performance features of the devices. Testing at the ETA engineering center consisted only of the conducted emissions test. Specific details concerning this test are discussed in the following subsection. All of this was done in accordance with Section 2.1.4 of the SAE scope document.

\subsubsection{Conducted Emissions}

The purpose for this test was to measure power line conductivity between all three devices: Intellon XAV101, Yitran IT700, and Maxim 2990/2991. This was done under stand-alone 120-Vac and 220-Vac voltages with a slave device acting as a load to the device under test.

The baseline is supposed to establish an average threshold between all three devices because it concerns the performance of isolation between the signal line and the power line for all three devices under test. Test setup instructions and drawings for the EMC tests conducted at the ETA engineering center are listed in Appendix B-5.

\subsection{Electromagnetic Communications Testing at Compliance Testing}

Compliance Testing is a local test center in Chandler, Arizona with experience in automotive and FCC EMC testing. The laboratory offered a test schedule that was favorable in adhering to the project timeline. The laboratory also demonstrated flexibility in setting up the needed tests and reporting the results. The staff at Compliance Testing demonstrated technical competence in operation of various test equipment and test procedures. The engineering team offered several suggestions in terms of procedure and the appropriate methods for obtaining correct results. The management team at Compliance Testing was forthcoming about the limitations of the laboratory's testing capability.

\section{RESULTS}

This section includes performance results for both the functional test and the EMC tests, respectively. Functional test results are categorized by product and by mode of testing (e.g., DC or alternating current $[\mathrm{AC}]$ testing). Specific details for each product and test mode are listed in the following subsections. 


\subsection{Functional Tests}

\subsubsection{Yitran IT700}

This product underwent two levels of functional AC testing. The primary level verified operability of the device, while the secondary level verified integration capability to a plug-in hybrid electric vehicle charger. The first test consisted of the product being tested using two laptops: one for the transmit module and the other for the receive module. The laptops had Yitran IT700 software to monitor and control the communication behavior of the devices. The modules were tested using the 120-Vac line and the Yitran power supply as well as using an external $12 \mathrm{~V}$ battery to separate the communication line from the provided power line used by the module. Data were successfully transferred at $7.5 \mathrm{kbps}$ over the power line. This was validated by an examination of the data report, as shown on the control interface in Figure 5. In preparation for EMC testing, Yitran engineering provided signal analysis reports. These are found in Appendix B-2. The display in Appendix B-1 shows device performance under 120 Vac with packet data being sent under FCC configuration. Functional test results for Yitran IT700 undergoing the secondary test are located in Appendix B-1. Data traffic shows a 50\% capacity at $7.5 \mathrm{kbps}$. The module showed virtually no performance difference between the first and the second test, which involved a $120-\mathrm{V}$ onboard charger.

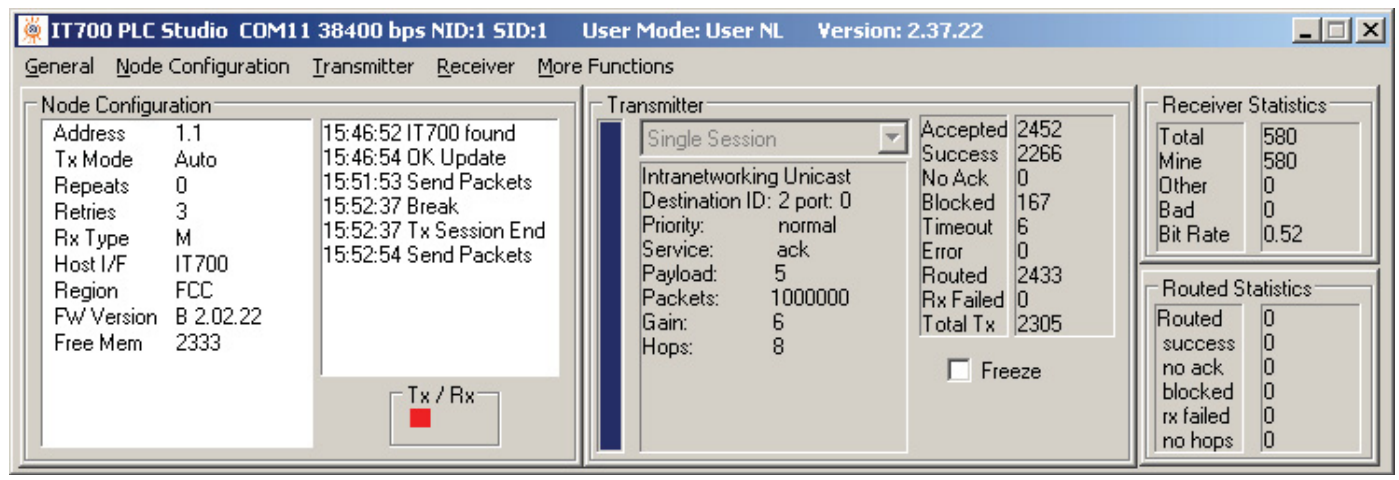

Figure 5. Data report screenshot for the Yitran IT700.

This product also underwent functional DC testing. Powered up with an external 12-V battery, the Yitran module was capable of communicating over the DC power line up to $250 \mathrm{~V}$ in its current configuration. However, as the current started to rise in those lines to charge the vehicle battery, the communication became completely blocked (see Appendix B-5 for details).

\subsubsection{Maxim 2990/2991}

For the functional AC testing, the Maxim 2990/2991, like the Yitran IT700, was tested using two laptops with vendor-supplied software to monitor communication. The modules were configured to be tested in either Cenelec A with Robo mode ON or Cenelec A with Robo mode OFF. Robo mode is a robust mode designed for configuration in harsh environments. The modules also can be configured in FCC mode with the option of having Robo mode ON or OFF. The Maxim 2990/2991 EV kit was tested under $120 \mathrm{Vac}, 220 \mathrm{Vac}$, with and without load, and using a 12-Vdc car battery to power up the modules. Data communication over the power line was validated using a console vendor-supplied application and an oscilloscope. A sample screen shot illustrating the console application is displayed in Figure 6. The screenshot illustrates, among others things, transmit data in bits per second and the success rate as indicated by ACK and ReTx indicators. The arrow indicates the packet count, based on a 7-BCD text string. A series of graphs illustrating data rates for both $120-\mathrm{Vac}$ and $220-\mathrm{Vac}$ load/no load conditions are displayed in Appendix B-3 (Maxim 2990/2991). All graphs represent FCC configuration with Robo mode ON. Robo mode illustrates the best case for device communication performance. 


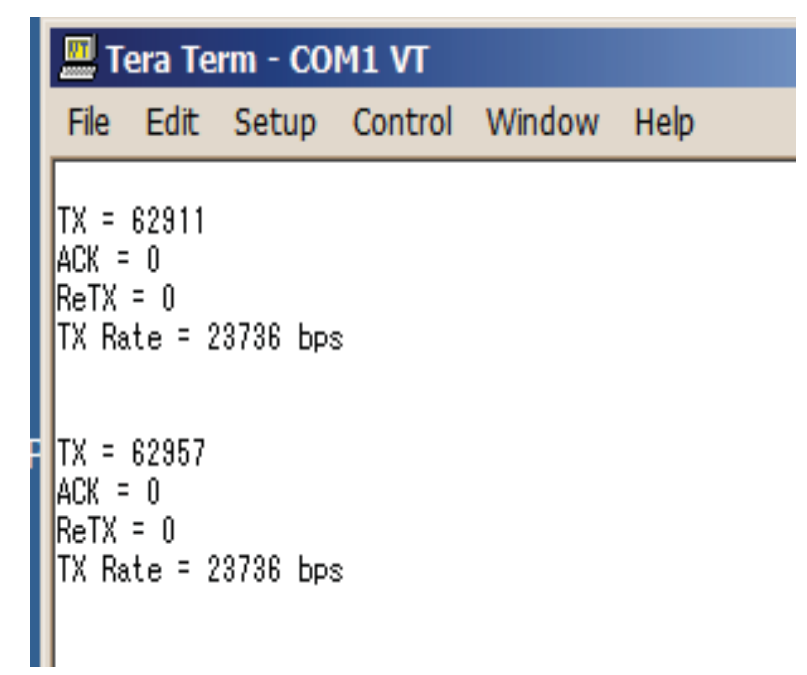

Figure 6. Screen shot for the Maxim 2990/2991 console.

For DC line testing, the Maxim EV kit was powered with an external $12-\mathrm{V}$ power supply and the product was able to communicate over the DC power line up to $250 \mathrm{~V}$ in its current configuration. As the current to charge the vehicle battery started to rise in those lines to charge the vehicle battery, the communication got completely blocked (see Appendix A-9 for details). With intervention of the Maxim engineer, it became possible to communicate on the high band of the FCC spectrum 300 to $450 \mathrm{kHz}$ (see Appendix B-9 for details).

\subsubsection{Intellon /Netgear XAV101}

The Intellon/Netgear XAV101 product differs from the Yitran and Maxim 2990/2991 in that it is sold as an active, approved consumer electronic device by meeting FCC Section 15, Part B. It is packaged as a Netgear product using TCP/IP communication over power line protocol. Testing the device requires two laptops with one module connected per laptop. The Netgear XAV101 runs under 135 to 400 -Vdc bus or 100 to $240 \mathrm{Vac}$. The robustness of this capability was tested using a large file measuring $942 \mathrm{MB}$ between two laptops and using a 180-Vdc bus, as well as a regular $110 \mathrm{Vac}$ and a $220 \mathrm{Vac}$ bus.

For the test over the AC line, the Homeplug AV device experienced no difficulty in communicating with a data rate of up to $50 \mathrm{Mbps}$. Typical data throughput was more around $35 \mathrm{Mbps}$ (Figure 7).

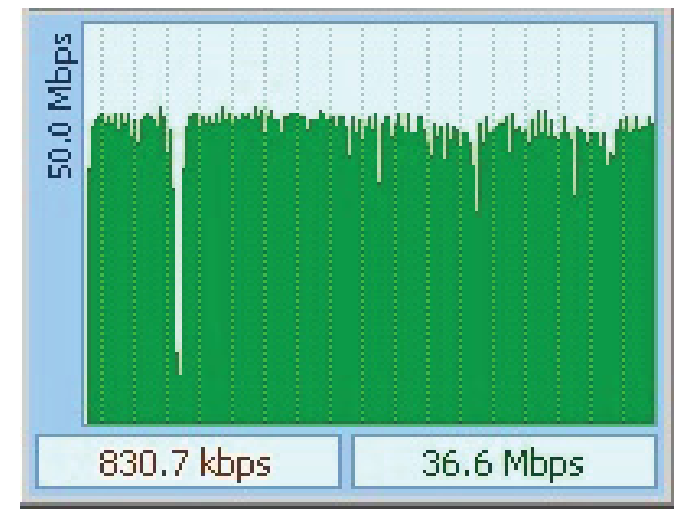

Figure 7. Homeplug AV data throughput over AC power line.

For the test over the DC line, because of the design of the module, it was not possible to test the XAV101 module with the same setup as the other two products. The device was communicating the majority of the time, while simultaneously charging at up $40 \mathrm{~A}$. The throughput was highly reduced (from 
tens of Mbps down to $80 \mathrm{kbps}$ ), Communication would occasionally be impossible during a full 10 -second period. Figure 8 shows the communication data throughput when the modules were installed on the output of the 25 analog front end Minit-Charger and the 200-V battery being charged at $25 \mathrm{~A}$.

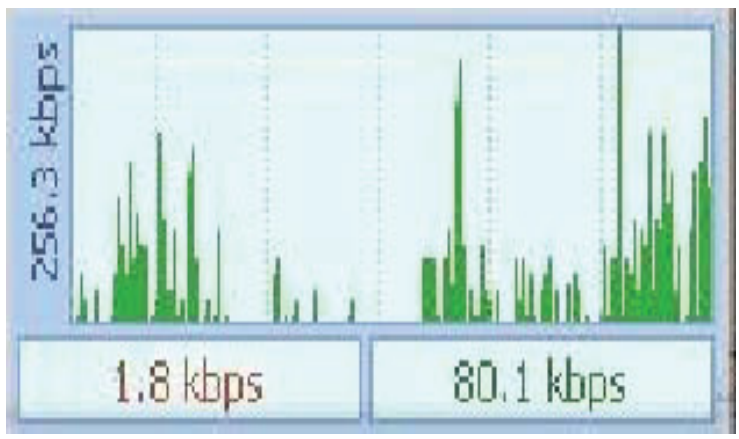

Figure 8. Homeplug AV data throughput over DC power line during fast charge.

\subsection{Baseline Electromagnetic Communications Test Results}

The EMC test results are separated by product family and test type. The different tests (such as AC or DC testing) were conducted separately. Before any testing of the modules could begin, a verification of the test setup needed to be carried out. Once this step was complete, the first module Yitran IT700 was tested, followed by Maxim 2990/2991, and finally the Intellon XAV101. The main series of tests performed were the conducted emissions tests, which were reported in graphical form for each product. Radiated emissions measurements also were performed but without an anechoic chamber; the results are of little value.

\subsubsection{Yitran IT700}

Testing began with a simple calibration of the test equipment in accordance with standard industry practices. Once the system was operational, testing began in the $120-\mathrm{Vac}$ mode followed by $220-\mathrm{Vac}$ mode. The performance of the Yitran IT700 under the conducted emissions baseline test with the device transmitting data is shown in Figure 9.

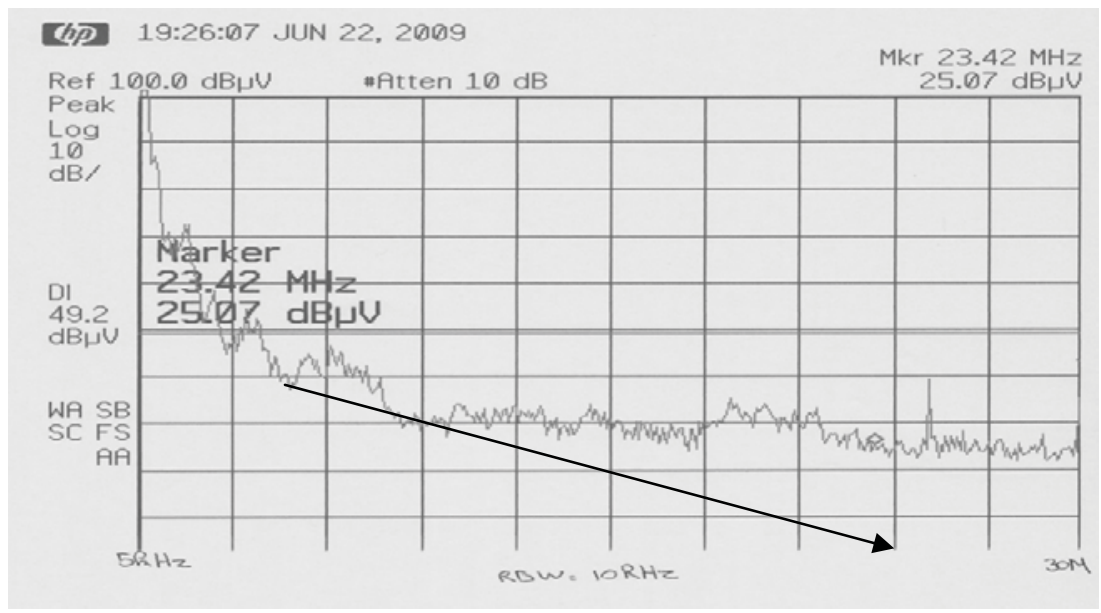

Figure 9. Yitran IT700 conducted emissions test results.

Figure 10 shows the conducted emissions measured when the device is not transmitting. Additionally, EMC testing results were performed in another laboratory under Yitran supervision to meet the current J551-5 EMC limitsError! Reference source not found. 


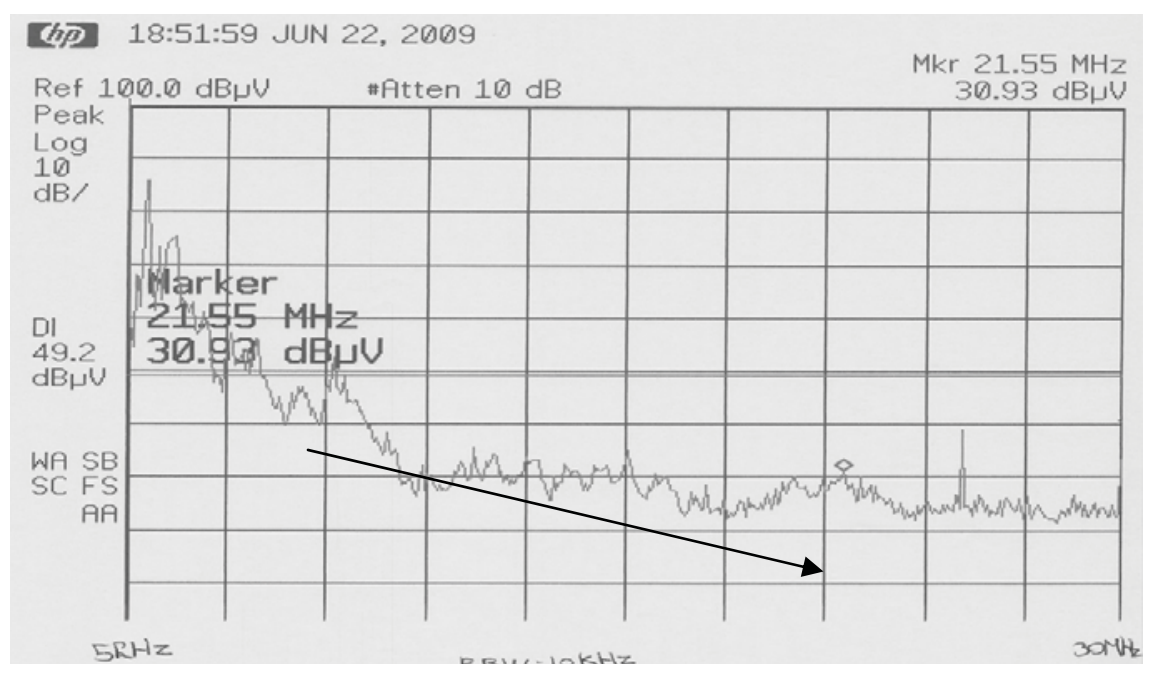

Figure 10. Yitran IT700 conducted emissions test results (no transmission).

\subsubsection{Maxim 2990/2991 (CPK Testing)}

Because the Maxim product entered the study later, ETA did not have the opportunity to test the conducted and radiated emission on the evaluation kit for this product. A supporting preparation for EMC testing was furnished by the engineering team at CPK testing. This was conducted under the direction of Maxim management, with feedback from ETA engineering. The results of the test cover radiated emissions in a graphical form and are listed in Appendix B-7. For the purposes of this report, the mode of operation under consideration is the FCC frequency (10 to $490 \mathrm{kHz}$ ) with Robo mode on. Figure 11 illustrates the radiated emissions test under the following frequency band from 30 to $242.4 \mathrm{MHz}$ ). 


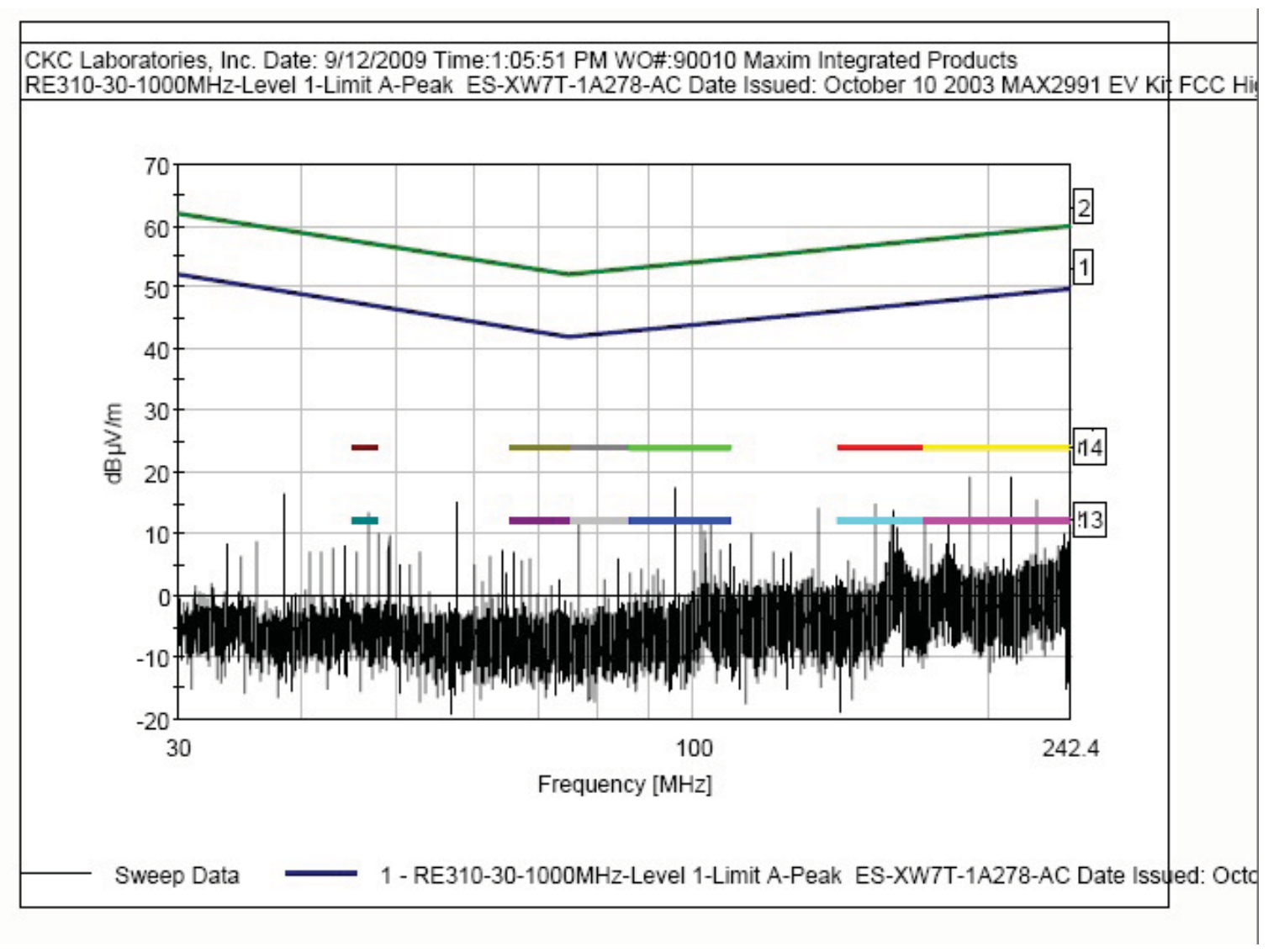

Figure 11. Radiated emissions test results for the Maxim 2990/2991 device.

The results provided by Maxim IC show that the device does not meet some of the Level 2 radiated emission requirements. However, it did not show any issue in meeting the Level 1 requirements, which is the level tested in this study.

\subsubsection{Intellon XAV101}

In a similar fashion to the Yitran IT700, the XAV101 was tested as a baseline for formal EMC testing. Because of the need for examining the similarities and differences with the Yitran IT700 product, conducted emission tests were performed at the ETA engineering center. Because the Intellon XAV101 operates over a higher and much wider band ( 2 to $28 \mathrm{MHz}$ ), graphical results will be different than those for the other products in the study. Figure 12 shows performance of the Intellon XAV101. 


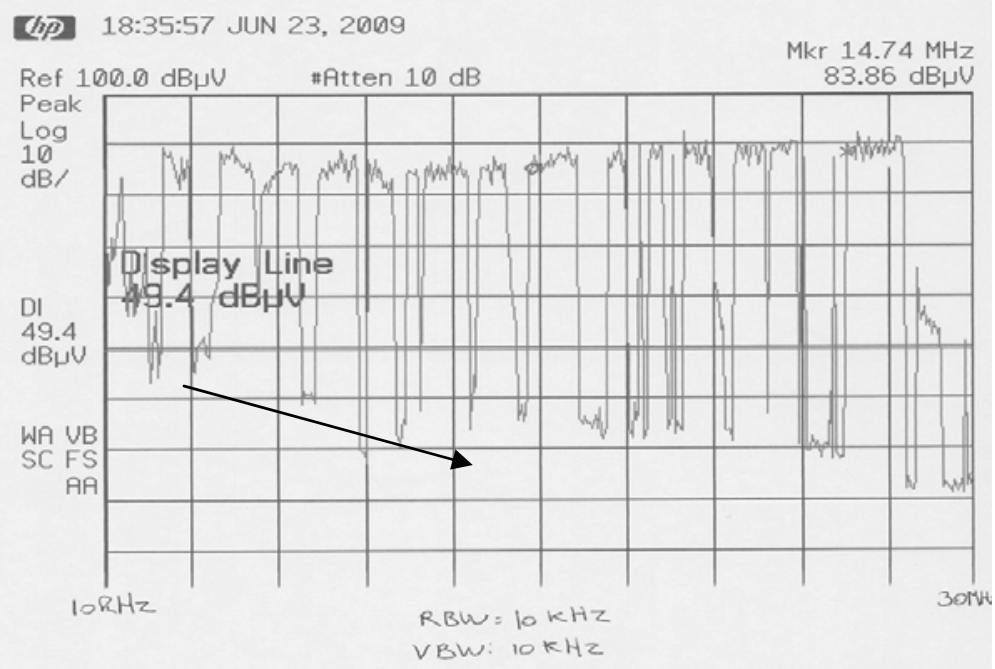

Figure 12. Intellon XAV101 conducted emissions test results.

A close examination of Figure 12 shows a higher rate of peaks covering the range of $10 \mathrm{kHz}$ to $30 \mathrm{Mhz}$ compared to the narrowband device from Yitran and Maxim. The signal characteristic represents a large file transferred between the two devices with a transmission control protocol/internet protocol. Conducted emissions of a Homeplug device are much higher than those recorded for the Yitran device on the 1.7 to $30-\mathrm{MHz}$ band. Note that this band is not being measured by the Ford CE420 test that was selected to test conducted emission; therefore, it might not be an issue for the Intellon device to meet the Ford EMC specifications.

\subsection{Formal Electromagnetic Communications Test Results}

All three devices underwent formal EMC testing at Compliance Testing. Compliance Testing is a licensed test facility specializing in FCC testing services. There were four tests that were conducted at Compliance Testing, starting with radiated emissions tests and followed by conducted emissions, bulk current injection, and radiated immunity tests.

The test center furnished a report covering specific results on all four test items. The test report is located in Appendix B-8. For the purposes of this study, the following sections will be directed at identifying the limitations of existing resources at the test laboratory and design challenges from the existing selection of products.

\subsection{Integration Challenges}

All three devices pose unique challenges because they apply to integration with the EV charger application. The EMC tests at Compliance Testing showed particular challenges with communication over the power line under a moderate frequency range of $150 \mathrm{kHz}$ to $30 \mathrm{MHz}$. All three devices exhibited a conducted signal gain in this region no matter the data rate or communication protocol. Of particular concern was the Intellon XAV101, which failed to meet FCC Part 15.107 Class B (a) in the 150-kHz to 30-MHz range, transmitting transmission control protocol/internet protocol packets at 1,500 bytes MTU, and also failed FCC Part 15.109, Table 1. Because the Intellon XAV101 product is already licensed under FCC Part $15 \mathrm{~B}$, its EMC performance was expected to show characteristics of a passing unit under typical operating conditions. However, given the general performance of all three devices, a general pattern of signal activity at various ranges on the frequency scale can be observed. The Maxim 2990/2991 and Yitran IT700 exhibited a signal gain below the accepted range as specified by the testing standard. The formal EMC report is located in Appendix B-8. 


\subsection{Test Laboratory Challenges}

The test laboratory was able to perform all four tests fairly well. Reporting of results was conducted in a professional manner under guidance of an experienced team of engineering professionals. Of particular concern was the limitation of not having a fully operational anechoic chamber. The FCC and Ford standards do not require it; they merely suggest it as a best practice. The alternative method is to use what is called an outside air testing chamber instead. The devices are placed in a tent structure while a high-gain antenna is placed outside. In order to capture accurate measurements, references are made to an average noise floor, which results in a test consisting of five points in place of a graphical display of results at various frequencies. The consequences of this result include the following:

- Repeatability - because the reporting of results is based on a noise floor, a repeat of the same test will produce different results due to the noise floor constantly changing.

- No graphical representation - because only five data points get captured, graphical representation becomes impractical. Using only five data points creates a false impression of the test performance over the entire test range. This omits important information regarding noise emissions under specific frequency bands within the test range.

\subsection{Performance Comparison}

All three products were tested under the same conditions and using the same methodology. Exceptions to this occurred regarding EMC testing at the ETA engineering center. The exception concerns the Maxim 2990/2991 product and is due to a late arrival and acceptance of prior EMC tests performed by the vendor's test center. The following items were observed in meeting comparative criteria as defined by Sections 2.1.2 and 2.1.5 of the SAE scope document.

\subsubsection{Radiated Performance}

The Intellon XAV101 has the highest data rate (up to $50 \mathrm{Mbps}$ ) compared to the Yitran IT700 (in the 3 to $7.5 \mathrm{kbps}$, depending what band is used) and Maxim 2990/2991 (in the 30 to $80 \mathrm{kbps}$, depending what band is used). The wireless behavior of the former at short range also was non-negligible. By connecting one Intellon device to a battery and the other to outlet power, the expectation would be that there would be no communication between the devices because no physical connection is made between the two devices. However, the results of this test showed that the two devices communicate at various lengths of separation. Figure 13 illustrates signal traffic between the Intellon devices at a separation distance of $2 \mathrm{ft}$. This was repeated on three occasions at this distance, with the devices facing each other, away from each other, and with one located at a height.

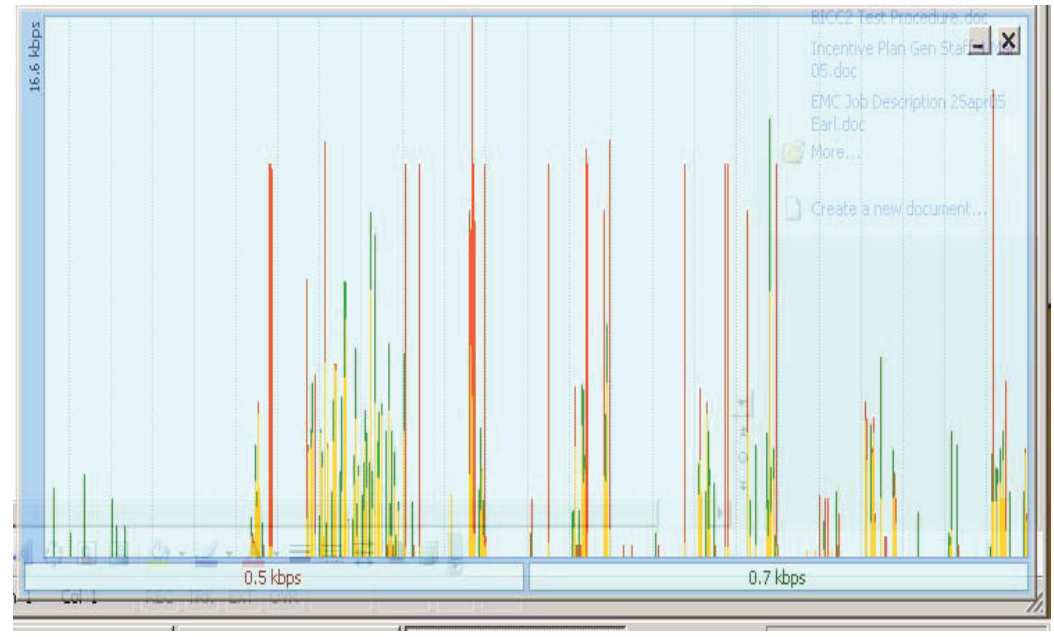

Figure 13. Wireless signal traffic results of the Intellon XAV101 product for a separation distance of $2 \mathrm{ft}$. 
In order to better characterize the performance of the radiating behavior, a repeat of the test was performed at 5-ft separation. The results are illustrated in Figure 14; the graph shows less traffic than what is shown in Figure 13, but still enough to warrant an observation. File transfer between the two test laptops was not successful, even though they were able to see each other. Perhaps, lack of communication between the two devices is due to the signal noise attributes of the apparent over-the-air communication. Details on functional test results for Intellon XAV101 can be found in Appendix B-4. Similar radio tests were performed with the Maxim and Yitran devices; however, those devices were not capable of transmitting over the air like the Intellon device.

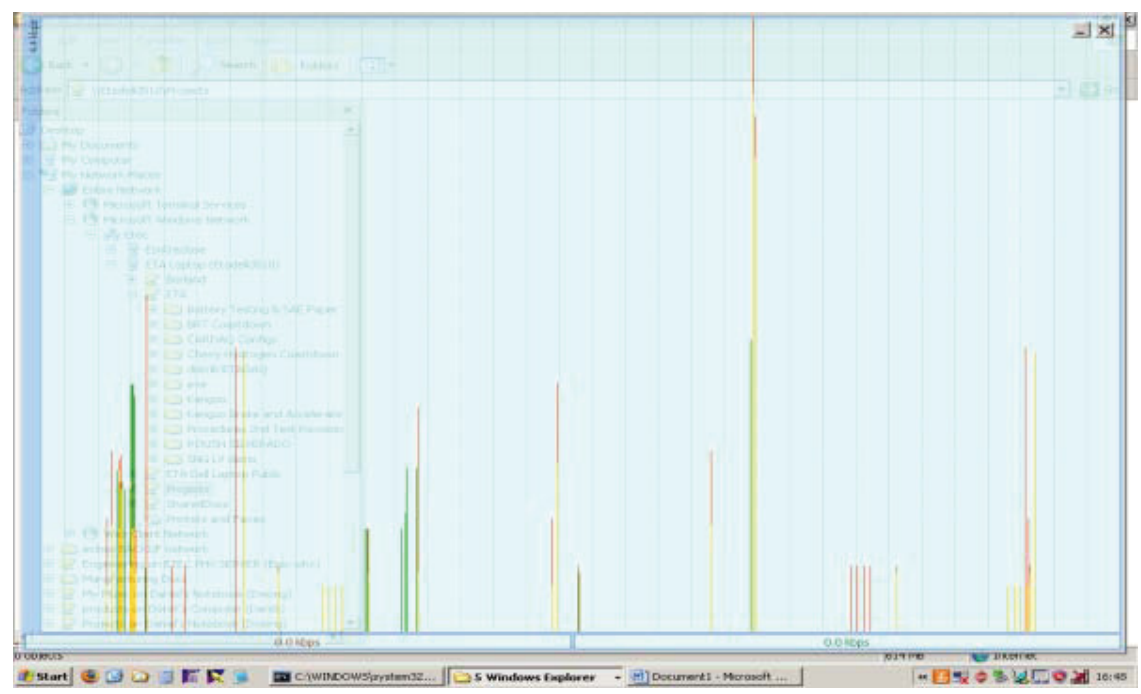

Figure 14. Wireless signal traffic results for the Intellon XAV101 product for a separation distance of $5 \mathrm{ft}$.

\subsubsection{Data Throughput}

The Yitran IT700 operated at the slowest speed $(7.5 \mathrm{kbps})$. Data transmission was slow but robust, although it failed to communicate over a DC power line when charging at $250 \mathrm{~A}$ with the Energy Technology Engineering Center industrial SC unit (see Appendix B-8 for details).

The Maxim 2990/2991 demonstrated an intermediate speed of $80 \mathrm{kbps}$ in the FCC range and $25 \mathrm{kbps}$ in the Cenelec A band. The analog front end module failed over DC. After some adjustments, the Maxim team successfully communicated over the DC power line when charging with the Energy Technology Engineering Center SC unit at $250 \mathrm{~A}$ at approximately $11 \mathrm{kbps}$ (see Appendix B-9 for details). Note that Maxim was the only candidate that had a chance to adapt their solution during field trials. Table 1 shows the different communication speeds observed for different test conditions with the three PLC solutions.

Table 1. Data rate comparison for the three programmable logic controller products.

\begin{tabular}{|l|c|c|c|c|}
\hline & $\begin{array}{c}\text { AC } \\
\text { (no load) }\end{array}$ & $\begin{array}{c}\text { AC } \\
\text { (32-A load) }\end{array}$ & $\begin{array}{c}\text { DC } \\
\text { (no load) }\end{array}$ & $\begin{array}{c}\text { DC } \\
\text { (250-A load)* }\end{array}$ \\
\hline IT700 (FCC) & $7.5 \mathrm{kbps}$ & $7.5 \mathrm{kbps}$ & $7.5 \mathrm{kbps}$ & 0 \\
\hline Maxim (ARIB) & $80 \mathrm{kbps}$ & $80 \mathrm{kbps}$ & $80 \mathrm{kbps}$ & $11 \mathrm{kbps} * *$ \\
\hline Intellon & $50 \mathrm{Mbps}$ & $50 \mathrm{Mbps}$ & $45 \mathrm{Mbps}$ & $* * *$ \\
\hline
\end{tabular}

* The PLC modules were connected over the DC lines of a 24-V industrial traction battery and an Energy Technology Engineering Center industrial SC charger.

** The Maxim evaluation kit was capable of communicating by tweaking the software and communicating on the high portion of the ARIB band only (see Appendix B-9 for details).

*** Because of the Netgear design, it was not possible to test the Intellon product with the Energy Technology Engineering Center SC charger. The Intellon product was tested with a smaller battery having a voltage of $180-\mathrm{V}$ nominal. It was clearly demonstrated that the data throughput was proportional to the current flowing in the line. Different tests with power sections led to very different data results, sometimes completely blocking the communication. 
- 60-ft AC line test - All three pairs of modules were individually set up on an isolated 60-ft line at $220-\mathrm{V}, 60-\mathrm{Hz}$ AC generated from a 110 to $220-\mathrm{V}$ step-up transformer. One unit was used as the transmitter while its opposite was used as the receiver. All three devices demonstrated good communication with no loss of signal.

- PLC communication while providing AC to an onboard charger - The Maxim 2990/2991 product was tested with a 30-A, 220-V draw that was charging an electric Mini Cooper. The engineering team from Maxim USA provided a report covering the performance of this test. The document can be found in Appendix B-9. The Yitran IT 700 and the Intellon XAV101 were tested earlier with a 12-A, $110-\mathrm{V}$ draw charging a converted plug-in hybrid Toyota Prius from Hymotion. All three technologies showed good signal transmission during charge events.

- PLC communication on industrial charger and off-board DC charger - Every solution had difficulty communicating over DC lines when high current was sent to the battery. Maxim was able to modify their product to enable it to run under one charger; however, this is charger-specific and in the real world with multiple charger vendors and changing technology, it might be undesirable. One alternative is to use the earth ground line as one of the communication lines. ETA has had good results using the battery positive line and earth ground wire to communicate with the Yitran and Maxim modules. The test could not be performed with the Intellon device because the communication and input power of the module cannot be separated.

\subsection{Cost Analysis}

A comparative cost analysis was done in support of Section 2.0 in the SAE scope document. The cost analyses consist of examining the integration capability of the technology under evaluation to the charger. Readiness of the product under evaluation to be integrated into a charger control system is examined in terms of hardware fit. The hardware fit must accommodate the basic function of the charger control system. However, there is no requirement that all features of the target product integrate if these features can be disabled without compromising the charger control system. Specific hardware criteria for each product under evaluation are discussed as follows:

- Development costs - Development costs consist of activities such as selecting a chip set, creating an engineering model, signal analysis studies, and PCB board layout. Items under this category include fabrication of prototypes, test validation plans, and manufacturability. For the purposes of this study, development costs will be limited to selection of a chipset and engineering model. The development costs for all four products under evaluation are listed in Appendix C-1.

- Part costs - The components needed for product manufacturing will be listed as the minimum required for integrating into the charger control system. All of this was done according to the chipset associated with each product. The Maxim and Yitran products operate exclusively under intended design criteria. The Intellon XAV101 operates as a Netgear product using a transmission control protocol/internet protocol. This will not be the protocol feature used in integrating this device to the charger control system. Instead, the part count will refer to the chipset features that better meet the needs of the current charger control systems most likely to be available on the market. The Zigbee product is a drop-in-place module requiring no component integration or redesign. It is for this reason there is no list of part costs involving this option. The part lists for the products are located in Appendix C-2.

- Support costs - In selecting a protocol technology, associated features will require additional support. This may consist of high-level software development for data capture, transmission, and storage. Support of point-of-sale service bundling, licensing, and other elements in support of media access also may be required. Given the wide array of features between all products under evaluation, a table is provided that lists rough costs for each product in Appendix C-3. 
For the purposes of this study, the cost analysis excludes time-to-market because this requires knowing details such as lead times on all components, scheduling, and manufacturing contracts. The cost analysis does offer a solid examination of feasibility in selecting one technology over another. The benefits of selecting a technology must include a readiness for deployment into an existing charger design. To select a technology without this consideration will result in added costs in designing a charger to accommodate the limits of the candidate technology.

Deployment costs will be lower if the most advantageous technology is selected. This was due to software integration, which was not part of the study, but was a contributing factor in development and deployment of the charging system. Deployment costs are assumed to encompass operability of the charger. Operability entails functional items such as frequency of charging, current draw, temperature, electrolyte levels, and state of charge for all available batteries. Optimization of operability will depend on market conditions, needs of the end user, and utility participation.

\section{CONCLUSIONS}

This study demonstrated some good results with PLC solutions in an AC-charging application where only one vehicle is being charged. No tests have been performed when multiple vehicles are being charged simultaneously. With multiple devices sharing the communication line (i.e., the same power line), the data throughput is expected to be divided by at least the number of additional devices added on the line. For example, the data rate of the Yitran solution would be reduced from 7.5 to $3.75 \mathrm{kbps}$ if two vehicles are being charged nearby and the traffic on the line is close to $100 \%$.

However, when DC charging, it was apparent that there was some difficulty in using a PLC; it is recommended that an earth ground be used as one of the signal lines for DC PLC communication in the specific context of battery charging.

The EMC testing showed no real problem in using any of the tested products in the United States; however, the SAE recently agreed on reaching a similar communication solution with the International Standards Organization and International Electrotechnical Commission. Requirements for Europe and Japan also need to be met; they were not part of this investigation. In fact, for example, current regulations in Japan forbid the use of a Homeplug AV device outside the home.

Cost implications in selecting candidate devices were measured by examining the manufacturability, communication front end support, and operability of the charger system. Manufacturability consists of what would be needed for full integration of the system. This includes an estimated bill of material, requirements of software design, and the potential impact of accommodating a smart meter. Front end support entails communication using front-end software development in accommodating the candidate protocol. Operability of the charger system consists of battery charging and monitoring while using a candidate device and its associated communication platform. The cost measure per candidate device for this category varied considerably, principally based on bandwidth features and communication speed. Currently, the chargers used by ETA do not require high-speed communication. If this were added on as an ancillary feature, it would impact the cost of the charger because additional hardware and software support would be required. The degree of front end support costs depend on the interface technology and chip set configuration. 
Appendix A

\section{Testing Descriptions}




\section{Appendix A-1 Setup Drawings for Validation}

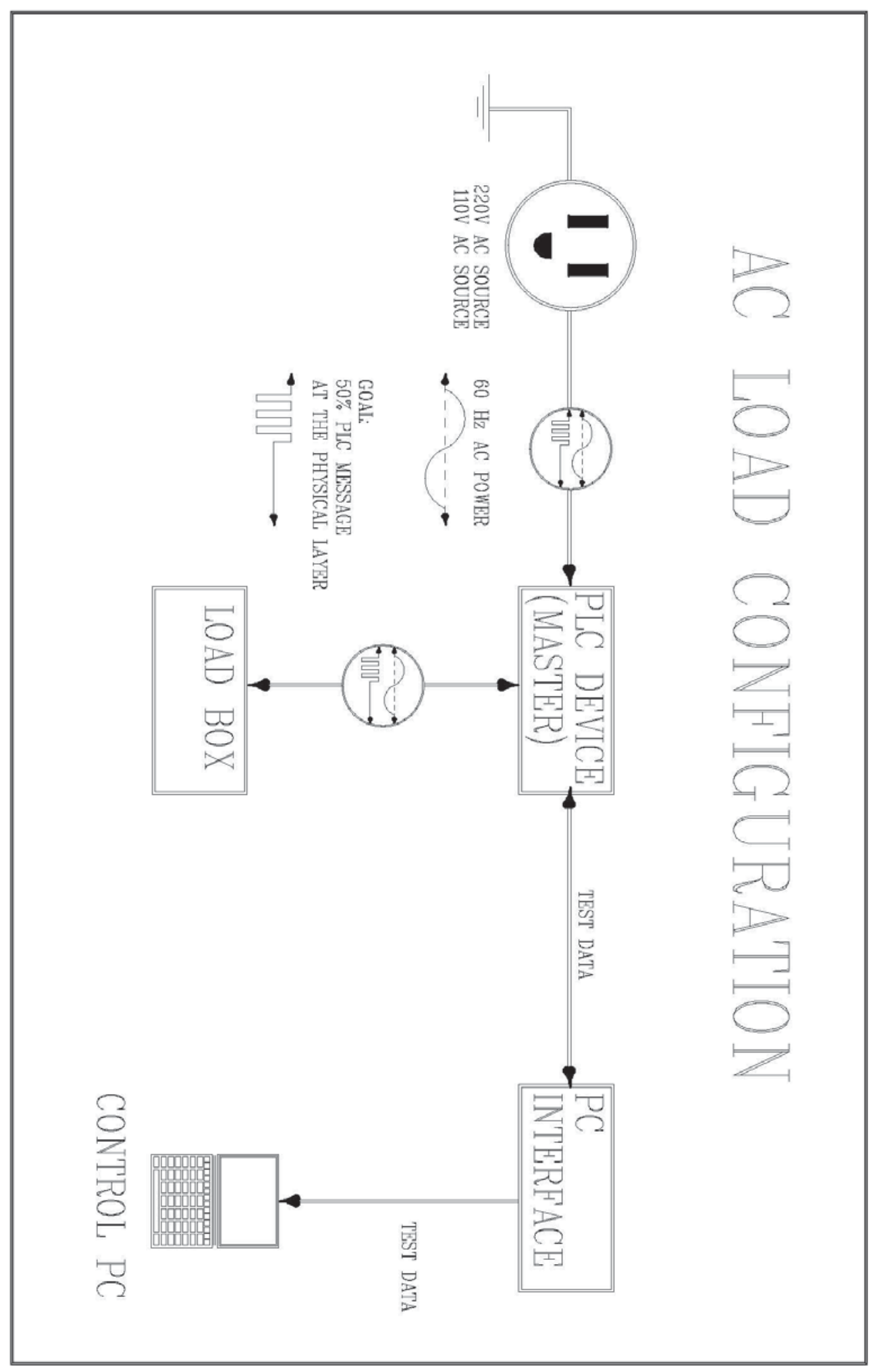




\section{Appendix A-2 Setup Instructions for Validation}

Preliminary: The purpose of this procedure is to define a process for measuring integration applicability of select communication devices, namely PLC solutions. The process must be unbiased in nature and yet show flexibility in meeting select requirements for a variety of charger applications.

\section{Mode of Operation}

- Suitable 110-V, 220-V, AC power

- $12-\mathrm{V}$ battery to power up the PLC device if possible

- Use paired device as load for the device under test

- Monitoring equipment appropriate for measuring the device under test

- Data collection procedures for transmitting and receiving data

- Record keeping procedures.

\section{Test Operation and Validation of Results}

- Develop procedures and methods for conducting various tests for repeatability.

- Examine and modify test procedures based on results and desired application.

- Validate and record for official record keeping purposes only those results that are repeatable under several test evolutions covering a wide time span.

- Be ready to terminate any test evolution if safety issues arise. Collect all data in preparation of a report. 


\section{Appendix A-3 \\ Microchip MRF24J40MA Data Sheets}

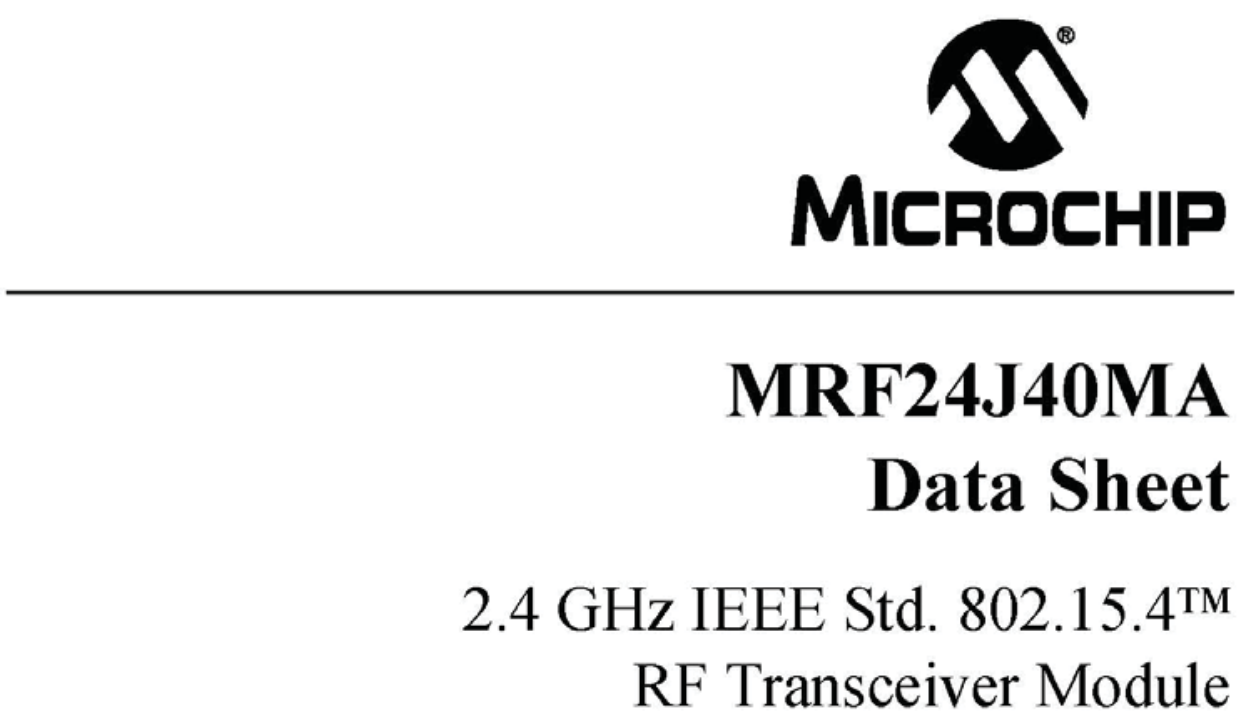


Note the following details of the code protection feature on Microchip devices:

- Microchip products meet the specification contained in their particular Microchip Data Sheet

- Microchip believes that its family of products is one of the most secure families of its kind on the market today, when used in the intended manner and under normal conditions.

- There are dishonest and possibly illegal methods used to breach the code protection feature. All of these methods, to our knowledge, require using the Microchip products in a manner outside the operating specifications contained in Microchip's Data Sheets. Most likely, the person doing so is engaged in theft of intellectual property.

- Microchip is willing to work with the customer who is concerned about the integrity of their code

- Neither Microchip nor any other semiconductor manufacturer can guarantee the security of their code. Code protection does not mean that we are guaranteeing the product as "unbreakable."

Code protection is constantly evolving. We at Microchip are committed to continuously improving the code protection features of our products. Attempts to break Microchip's code protection feature may be a violation of the Digital Millennium Copyright Act. If such acts allow unauthorized access to your software or other copyrighted work, you may have a right to sue for relief under that Act.

Information contained in this publication regarding device applications and the like is provided only for your convenience and may be superseded by updates. It is your responsibility to ensure that your application meets with your specifications MICROCHIP MAKES NO REPRESENTATIONS OR WARRANTIES OF ANY KIND WHETHER EXPRESS OR IMPLIED, WRITTEN OR ORAL, STATUTORY OR OTHERWISE, RELATED TO THE INFORMATION, OTHERISE BUT NOT LIMITED TO ITS CO INCLUDING BUT NOT LIMITED TO ITS CONDITION HANTABILITY OR FITNESS FOR PURPOSE. Microchip disclaims all liability arising from this information and its use. Use of Microchip devices in life support and/or safety applications is entirely at the buyer's risk, and the buyer agrees to defend, indemnify and hold harmless Microchip from any and all damages, claims, hold har or exs conv, or expences resuling intellectual property rights.

\section{Trademarks}

The Microchip name and logo, the Microchip logo, Accuron, dSPIC, KEELOQ, KEELOQ logo, MPLAB, PIC, PICmicro, PICSTART, IfPIC, SmartShunt and UNI/O are registered trademarks of Microchip Technology Incorporated in the USA and other countries.

FilterLab, Linear Active Thermistor, MXDEV, MXLAB SEEVAL, SmartSensor and The Embedded Control Solutions Company are registered trademarks of Microchip Technology Incorporated in the U.S.A.

Analog-for-the-Digital Age, Application Maestro, CodeGuard dsPICDEM, dsPICDEM net, dsPICworks, dsSPEAK, ECAN ECONOMONITOR, FanSense, In-Circuit Serial

Programming, ICSP, ICEPIC, Mindi, MiWi, MPASM, MPLAB Certified logo, MPLIB, MPLINK, mTouch, PICKit, PICDEM, PICDEM net, PICtail, PIC ${ }^{32}$ logo, PowerCal, Powerinfo PowerMate, PowerTool, REAL ICE, rfLAB, Select Mode, Total Endurance, WiperLock and ZENA are trademarks of Microchip Technology Incorporated in the U.S.A. and other countries.

SQTP is a service mark of Microchip Technology Incorporated in the U.S.A.

All other trademarks mentioned herein are property of their respective companies

(C) 2008. Microchip Technology Incorporated, Printed in the U.S.A., All Rights Reserved.

E Printed on recycled paper

\section{QUALITY MANAGEMENT SYSTEM CERTIFIED BY DNV =ISO/TS 16949:2002}

Microchip receivediso/TS-16949:2002 certification for its woridwide headquarters, design and wafer fabrication facilities in Chandiler and and india. The Company's quality system processes and procedure are for its PIC MCUS and dSPIC OSCS, KEELOQ⿻ COde hopping devices, Serial EEPROMS, microperipherals, nonvolatile memory and analog products. In addition, Microchip's quality system for the design 


\subsection{GHz IEEE Std. 802.15.4 ${ }^{\mathrm{TM}}$ RF Transceiver Module}

\section{Features:}

- IEEE Std. 802.15.4 $4^{\mathrm{m}}$ Compliant RF Transceiver

- Supports ZigBee ${ }^{\oplus}, M_{i W i}{ }^{m}, M_{1} W_{i}{ }^{m}$ P2P and Proprietary Wireless Networking Protocols

- Small Size: $0.7 "$ x 1.1" (17.8 mm x $27.9 \mathrm{~mm})$, Surface Mountable

- Integrated Crystal, Internal Voltage Regulator. Matching Circuitry and PCB Antenna

- Easy Integration into Final Product - Minimize Product Development, Quicker Time to Market

- Radio Regulation Certification for United States (FCC), Canada (IC) and Europe (ETSI)

- Compatible with Microchip Microcontroller Families (PIC16F, PIC18F, PIC24F/H, dsPIC33 and PIC32)

- Up to $400 \mathrm{ft}$. Range

Operational:

- Operating Voltage: $2.4-3.6 \mathrm{~V}$ (3.3V typical)

- Temperature Range: $-40^{\circ} \mathrm{C}$ to $+85^{\circ} \mathrm{C}$ Industrial

- Simple, Four-Wire SPI Interface

- Low-Current Consumption:

- RX mode: 19 mA (typical)

- TX mode: 23 mA (typical)

- Sleep: $2 \mu \mathrm{A}$ (typical)
RF/Analog Features:

- ISM Band 2.405-2.48 GHz Operation

- Data Rate: 250 kbps

- $-94 \mathrm{dBm}$ Typical Sensitivity with $+5 \mathrm{dBm}$ Maximum Input Level

- +0 dBm Typical Output Power with

36 dB TX Power Control Range

- Integrated Low Phase Noise VCO, Frequency

Synthesizer and PLL Loop Filter

- Digital VCO and Filter Calibration

- Integrated RSSI ADC and I/Q DACs

- Integrated LDO

- High Receiver and RSSI Dynamic Range

\section{MAC/Baseband Features:}

- Hardware CSMA-CA Mechanism, Automatic ACK Response and FCS Check

- Independent Beacon, Transmit and GTS FIFO

- Supports all CCA modes and RSS/LQI

- Automatic Packet Retransmit Capable

- Hardware Security Engine (AES-128) with CTR, $\mathrm{CCM}$ and $\mathrm{CBC}-\mathrm{MAC}$ modes

- Supports Encryption and Decryption for MAC

Sublayer and Upper Layer

FIGURE 1: $\quad$ PIN DIAGRAM 


\section{MRF24J40MA}

\section{Table of Contents}

1.0 Device Overview

2.0 Circuit Description.

3.0 Regulatory Approval..

4.0 Electrical Characteristics

Appendix A: Revision History

index

The Microchip Web Site

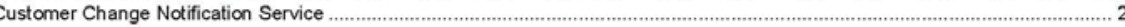

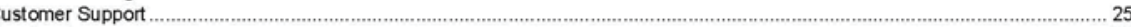

Reader Response

Product Identification System.

\section{TO OUR VALUED CUSTOMERS}

It is our intention to provide our valued customers with the best documentation possible to ensure successful use of your Microchip products. To this end, we will continue to improve our publications to better suit your needs. Our publications will be refined and enhanced as new volumes and updates are introduced.

If you have any questions or comments regarding this publication. please contact the Marketing Communications Department via E-mail at docerrors@microchip.com or fax the Reader Response Form in the back of this data sheet to (480) $792-4150$. We welcome your feedback.

\section{Most Current Data Sheet}

To obtain the most up-to-date version of this data sheet. please register at our Worldwide Web site at: http://www.microchip.com

You can determine the version of a data sheet by examining its literature number found on the bottom outside corner of any page. The last character of the literature number is the version number. (e.g.. DS30000A is version A of document DS30000).

\section{Errata}

An errata sheet, describing minor operational differences from the data sheet and recommended workarounds. may exist for current devices. As device/documentation issues become known to us, we will publish an errata sheet. The errata will specify the revision of silicon and revision of document to which it applies.

To determine if an errata sheet exists for a particular device, please check with one of the following:

- Microchip's Worldwide Web site: http:INwww.microchip.com

- Your local Microchip sales office (see last page)

When contacting a sales office, please specify which device, revision of silicon and data sheet (include literature number) you are using.

Customer Notification System

Register on our web site at www.microchip.com to receive the most current information on all of our products. 


\subsection{DEVICE OVERVIEW}

The MRF24J4OMA is a $2.4 \mathrm{GHz}$ IEEE Std. $802.15 .4^{\mathrm{TM}}$ compliant, surface mount module with integrated crystal, internal voltage regulator, matching circuitry and PCB antenna. The MRF24J40MA module operates in the non-licensed $2.4 \mathrm{GHz}$ frequency band and is FCC, IC and ETSI compliant. The integrated module design frees the integrator from extensive RF and antenna design, and regulatory compliance testing, allowing quicker time to market.

The MRF24J40MA module is compatible with Microchip's ZigBee ${ }^{\circledR}, M_{i} W_{i}{ }^{m}$ and MiWi P2P software stacks. Each software stack is available as a free download, including source code, from the Microchip web site http:///Www. microchip.com/wireless.

The MRF24J40MA module has received regulatory approvals for modular devices in the United States (FCC), Canada (IC) and Europe (ETSI). Modular approval removes the need for expensive RF and antenna design and allows the end user to place the
MRF24J40MA module inside a finished product and not require regulatory testing for an intentional radiator (RF transmitter). See Section 3.0 "Regulatory Approval" for specific requirements to be followed by the integrator

\subsection{Interface Description}

Figure 1-1 shows a simplified block diagram of the MRF24J40MA module. The module is based on the Microchip Technology MRF24J40 IEEE $802.15 .4^{\mathrm{m} M}$ $2.4 \mathrm{GHz}$ RF Transceiver IC. The module interfaces to many popular Microchip $\mathrm{PIC}^{\oplus}$ microcontrollers via a 4-wire serial SPI interface, interrupt, wake, Reset, power and ground, as shown in Figure 1-2. Table 1-1 provides the pin descriptions.

Data communications with the MRF24J40MA module are documented in the "MRF24J40 IEEE 802.15.4" 2.4 GHz RF Transceiver Data Sheet" (DS39776). Refer to the MRF24J40 Data Sheet for specific serial interface protocol and register definitions.

\section{FIGURE 1-1: $\quad$ MRF24J4OMA BLOCK DIAGRAM}

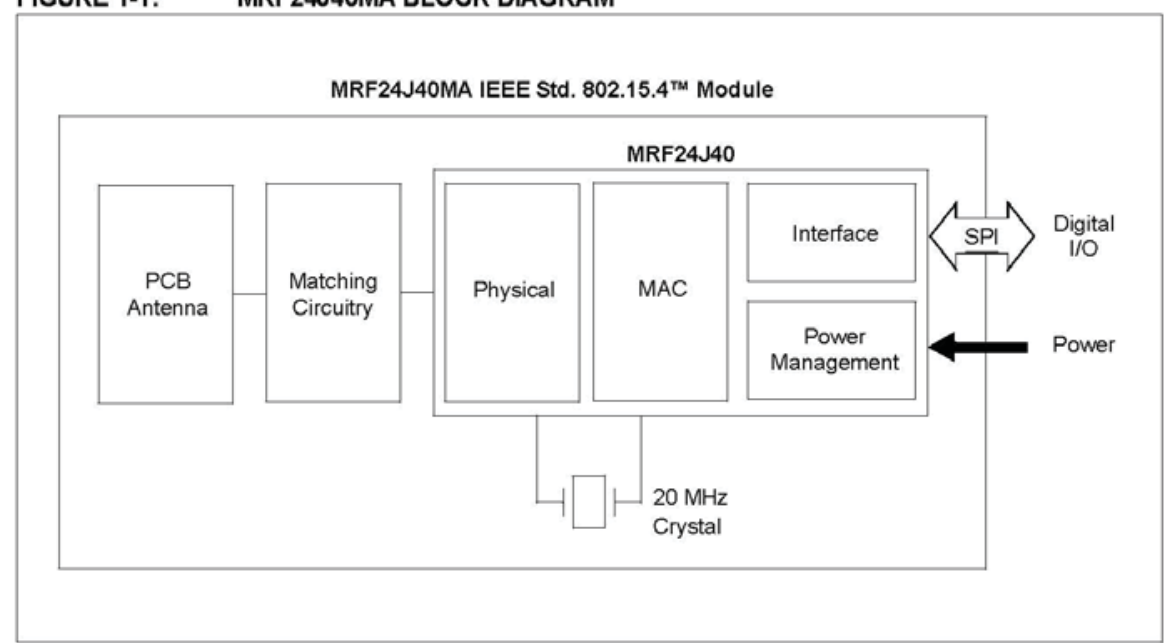


MRF24J40MA

TABLE 1-1: PIN DESCRIPTION

\begin{tabular}{|c|c|c|l|}
\hline Pin & Symbol & Type & \\
\hline \hline 1 & GND & Power & Ground \\
\hline 2 & $\overline{\text { RESET }}$ & $\mathrm{DI}$ & Global hardware Reset pin \\
\hline 3 & WAKE & $\mathrm{DI}$ & External wake-up trigger \\
\hline 4 & INT & DO & Interrupt pin to microcontroller \\
\hline 5 & SDI & DI & Serial interface data input \\
\hline 6 & SCK & DI & Serial interface clock \\
\hline 7 & SDO & DO & Serial interface data output from MRF24J40 \\
\hline 8 & $\overline{\text { CS }}$ & DI & Serial interface enable \\
\hline 9 & NC & - & No connection (allow pin to float; do not connect signal) \\
\hline 10 & VIN & Power & Power supply \\
\hline 11 & GND & Ground & Ground \\
\hline 12 & GND & Ground & Ground \\
\hline
\end{tabular}

Legend: Pin type abbreviation: $\mathrm{D}=$ Digital, $\mathrm{I}=$ Input, $\mathrm{O}=$ Output

FIGURE 1-2: $\quad$ MICROCONTROLLER TO MRF24J4OMA INTERFACE

$\mathrm{PIC}^{3} \mathrm{MCU} \quad$ MRF24J40MA

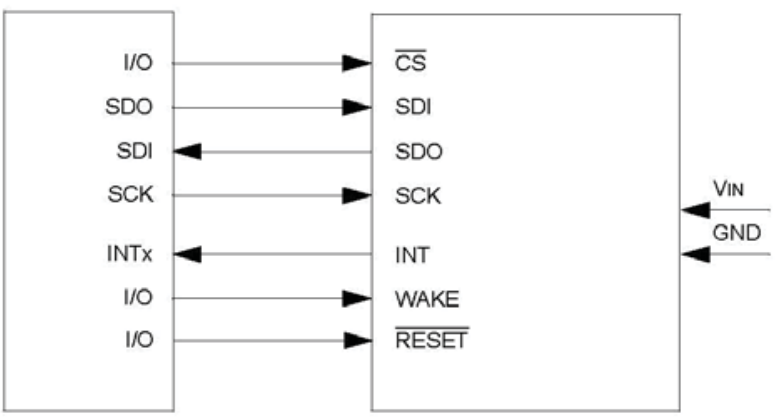


MRF24J40MA

\subsection{Mounting Details}

The MRF24J40MA is a surface mountable module. Module dimensions are shown in Figure 1-3. The module Printed Circuit Board (PCB) is 0.032 " thick with castellated mounting points on the edge. Figure 1-4 is a recommended host PCB footprint for the MRF24J40MA.

The MRF24J40MA has an integrated PCB antenna. For the best performance, follow the mounting details shown in Figure 1-5. It is recommended that the module be mounted on the edge of the host PCB, and an area around the antenna, approximately $1.2^{\prime \prime}$, be kept clear of metal objects. A host PCB ground plane around the MRF24J40MA acts as a counterpoise to the $\mathrm{PCB}$ antenna. It is recommended to extend the ground plane at least 0.4 " around the module.

\section{FIGURE 1-3: MODULE DETAILS}

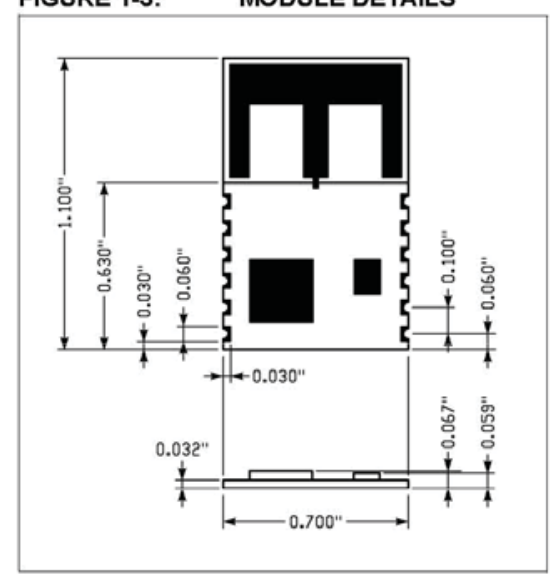

\section{FIGURE 1-4: RECOMMENDED PCB} FOOTPRINT

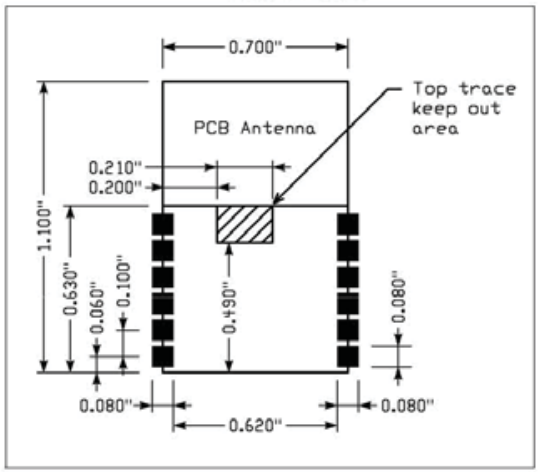


MRF24J40MA

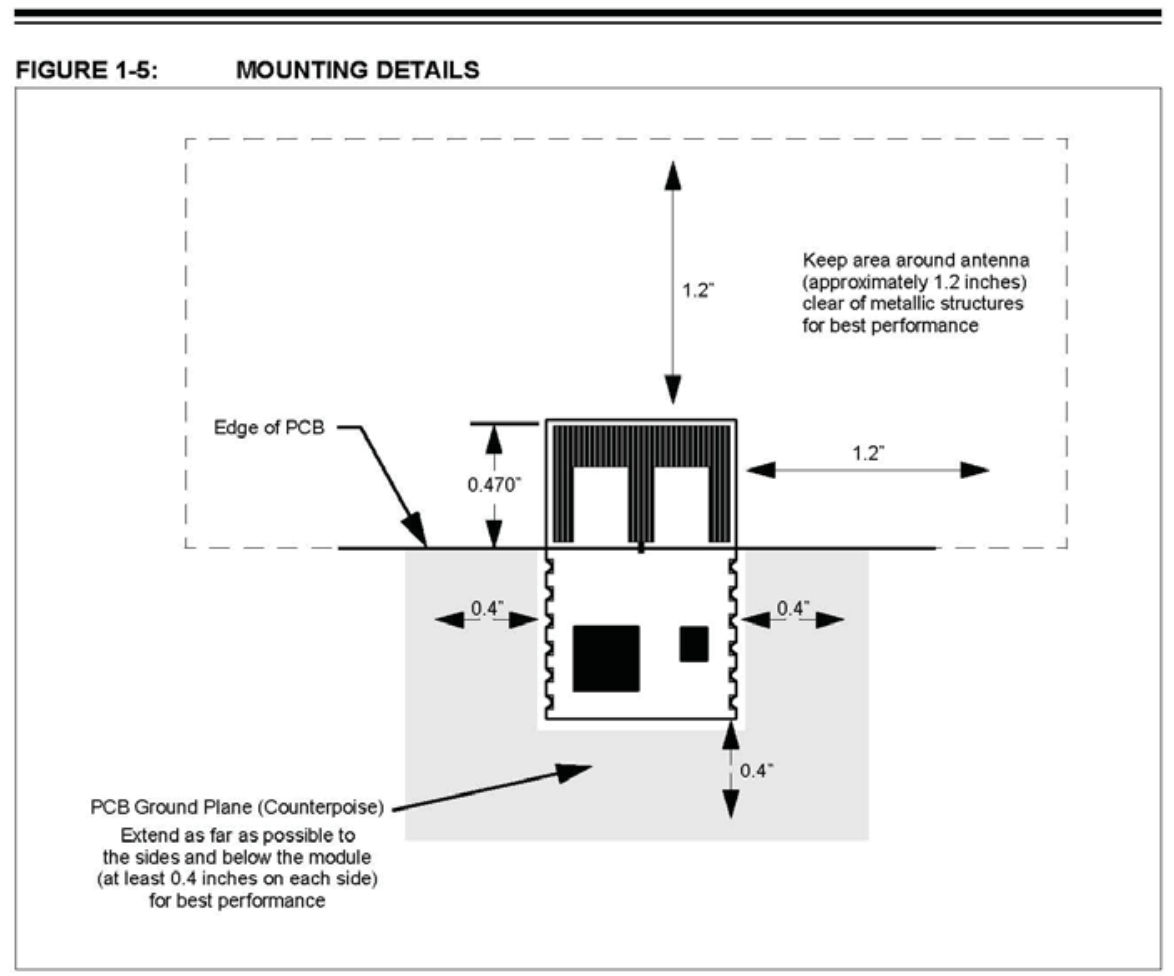

DS70329B-page 6

2008 Microchip Technology Inc. 


\section{MRF24J40MA}

\subsection{CIRCUIT DESCRIPTION}

The MRF24J40MA is a complete $2.4 \mathrm{GHz}$ IEEE Std. 802.15.4 $4^{\mathrm{TM}}$ compliant surface mount module with integrated crystal, internal voltage regulator, matching circuitry and PCB antenna. The MRF24J40MA module interfaces to many popular Microchip PIC microcontrollers via a 4-wire serial SPI interface, interrupt, wake, Reset, power and ground. Data communications with the MRF24J40MA module are documented in the "MRF24J40 IEEE 802.15.4" $2.4 \mathrm{GHz}$ RF Transceiver Data Sheef' (DS39776). Refer to the MRF24J40 Data Sheet for specific serial interface protocol and register definitions.

\subsection{Schematic}

A schematic diagram of the module is shown in Figure 2-1 and the Bill of Materials (BOM) is shown in Table 2-1.

The MRF24J40MA module is based on the Microchip Technology MRF24J40 IEEE $802.15 .4^{\mathrm{TM}} 2.4 \mathrm{GHz}$ RF Transceiver IC. The serial I/O (SCK, SDI, SDO and $\overline{\mathrm{CS}}), \overline{\mathrm{RESET}}$, WAKE and INT pins are brought out to the module pins. The SDO signal is tri-state buffered by IC2 to solve a silicon errata, where the SDO signal does not release to a high-impedance state, after the $\overline{\mathrm{CS}}$ pin returns to its inactive state. Crystal, $\mathrm{X} 1$, is a $20 \mathrm{MHz}$ crystal with a frequency tolerance of $\pm 10 \mathrm{ppm} @ 25^{\circ} \mathrm{C}$ to meet the IEEE Std. 802.15 .4 symbol rate tolerance of $\pm 40 \mathrm{ppm}$. A balun is formed by components: L1, L3, C2 and C14. L2 is an RF choke and pull-up for the RFP and RFN pins on the MRF24J40. C15 is a DC block capacitor. A low-pass filter is formed by components: $L 4, C 16$ and $C 17$. The remaining capacitors provide RF and digital bypass. 
MRF24J40MA

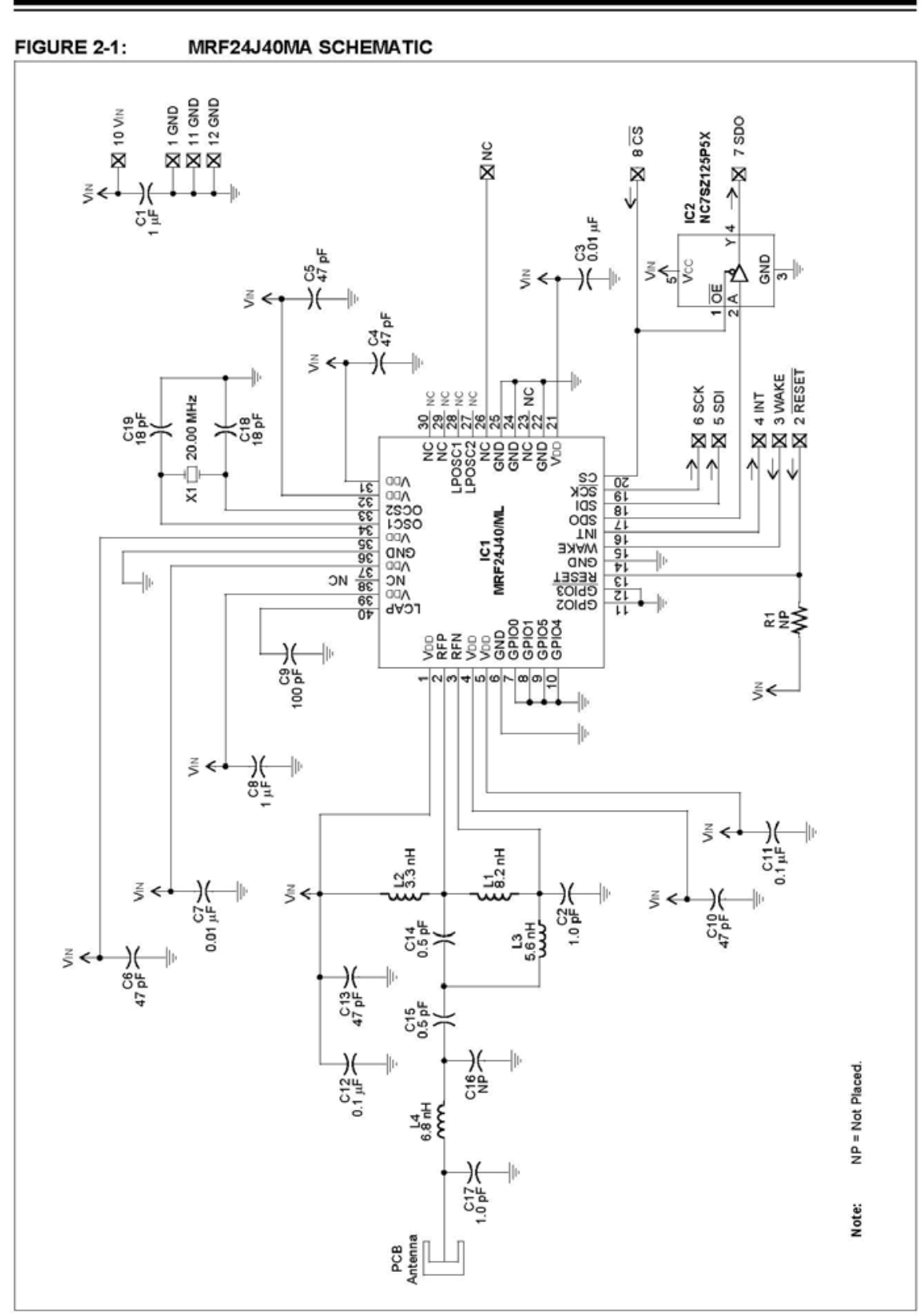

DS70329B-page 8

Advance Information

\2008 Microchip Technology Inc 
MRF24J40MA

TABLE 2-1: MRJ24J4OMA BILL OF MATERIALS

\begin{tabular}{|c|c|c|c|}
\hline Designator & Description & Manufacturer & Part Number \\
\hline C1 & Chip Capacitor 0402 X5R $1 U$ & Murata & GRM155R60J105ME19D \\
\hline $\mathrm{C} 2$ & Chip Capacitor 0402 COG 1.0P & Murata & GRM1555C1H1R0CZ01D \\
\hline $\mathrm{C} 3$ & Chip Capacitor 0402 X7R 10N & Murata & GRM155R71E103KA01D \\
\hline $\mathrm{C} 4$ & Chip Capacitor 0402 COG 47P & Murata & GRM1555C1H470JZ01D \\
\hline C5 & Chip Capacitor 0402 COG 47P & Murata & GRM1555C1H470JZ01D \\
\hline $\mathrm{C} 6$ & Chip Capacitor 0402 COG 47P & Murata & GRM1555C1H470JZ01D \\
\hline C7 & Chip Capacitor 0402 X7R 10N & Murata & GRM155R71E103KA01D \\
\hline $\mathrm{C} 8$ & Chip Capacitor 0402 X5R $1 U$ & Murata & GRM155R60J105ME19D \\
\hline $\mathrm{C9}$ & Chip Capacitor 0402 COG 100P & Murata & GRM1555C1H101JZ01D \\
\hline C10 & Chip Capacitor 0402 COG 47P & Murata & GRM1555C1H470JZ01D \\
\hline C11 & Chip Capacitor 0402 X5R $100 \mathrm{~N}$ & Murata & GRM155R61A104KA01D \\
\hline $\mathrm{C} 12$ & Chip Capacitor 0402 X5R $100 \mathrm{~N}$ & Murata & GRM155R61A104KA01D \\
\hline $\mathrm{C} 13$ & Chip Capacitor 0402 COG 47P & Murata & GRM1555C1H470JZ01D \\
\hline $\mathrm{C} 14$ & Chip Capacitor 0402 COG 0.5P & Murata & GRM1555C1HR50CZ01D \\
\hline C15 & Chip Capacitor 0402 COG 0.5P & Murata & GRM1555C1HR50CZ01D \\
\hline C16 & Not Placed & & \\
\hline C17 & Chip Capacitor 0402 COG 1.0P & Murata & GRM1555C1H1R0CZ01D \\
\hline C18 & Chip Capacitor 0402 COG 18P & Murata & GRM1555C1H180JZ01D \\
\hline C19 & Chip Capacitor 0402 COG 18P & Murata & GRM1555C1H180JZ01D \\
\hline IC1 & IEEE $802.15 .4^{\mathrm{mM}}$ RF Transceiver & Microchip & MRF24J40-I/ML \\
\hline IC2 & Buffer, SC70 Package & Fairchild & NC7SZ125P5X \\
\hline L1 & Chip Inductor $04028.2 \mathrm{~N}$ & Panasonic & ELJ-RF8N2JFB \\
\hline L2 & Chip Inductor $04023.3 \mathrm{~N}$ & Panasonic & ELJ-RF3N3DFB \\
\hline L3 & Chip Inductor $04025.6 \mathrm{~N}$ & Panasonic & ELJ-RF5N6DFB \\
\hline L4 & Chip Inductor $04026.8 \mathrm{~N}$ & Panasonic & ELJ-RF6N8JFB \\
\hline R1 & Not Placed & & \\
\hline $\mathrm{X} 1$ & $20 \mathrm{MHz}$ Crystal & Abracon & ABM8-156-20.0000MHZ-T \\
\hline
\end{tabular}




\section{MRF24J40MA}

\subsection{Printed Circuit Board}

The MRF24J40MA module printed circuit board is constructed with FR4 material, four layers and

0.032 inches thick. The layers are shown in Figure 2-2

through Figure 2-6. The stack up of the PCB is shown in Figure 2-7.

FIGURE 2-2: TOP SILK SCREEN

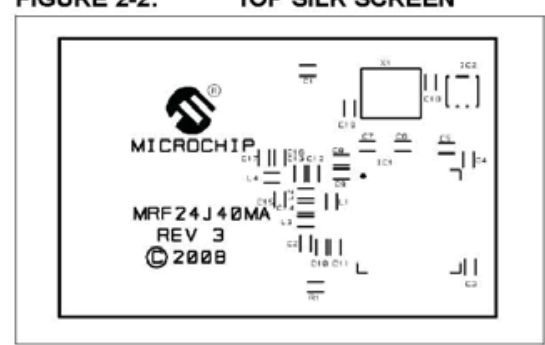

\section{FIGURE 2-3: $\quad$ TOP COPPER}

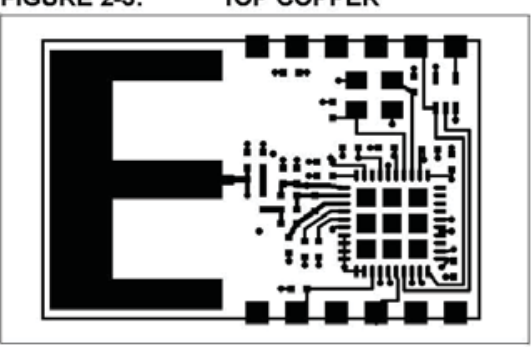

FIGURE 2-4: $\quad$ LAYER 2 - GROUND PLANE

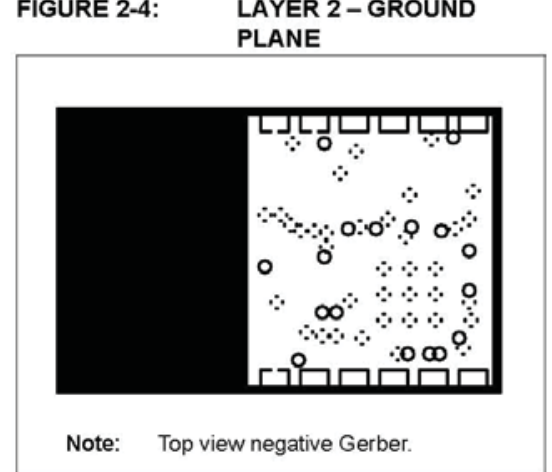

Note: Top view negative Gerber

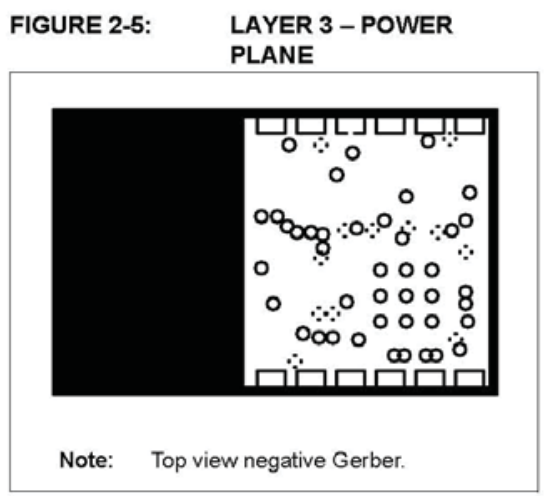

FIGURE 2-6: $\quad$ BOTTOM COPPER

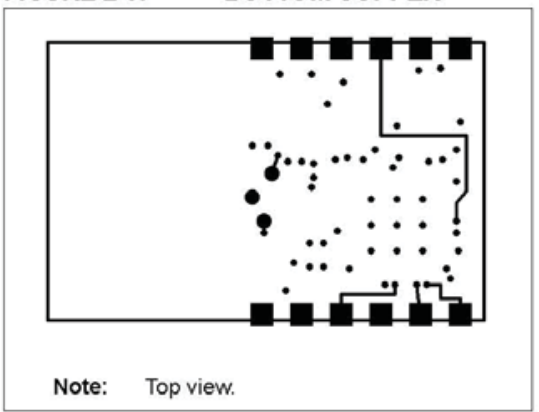


MRF24J40MA

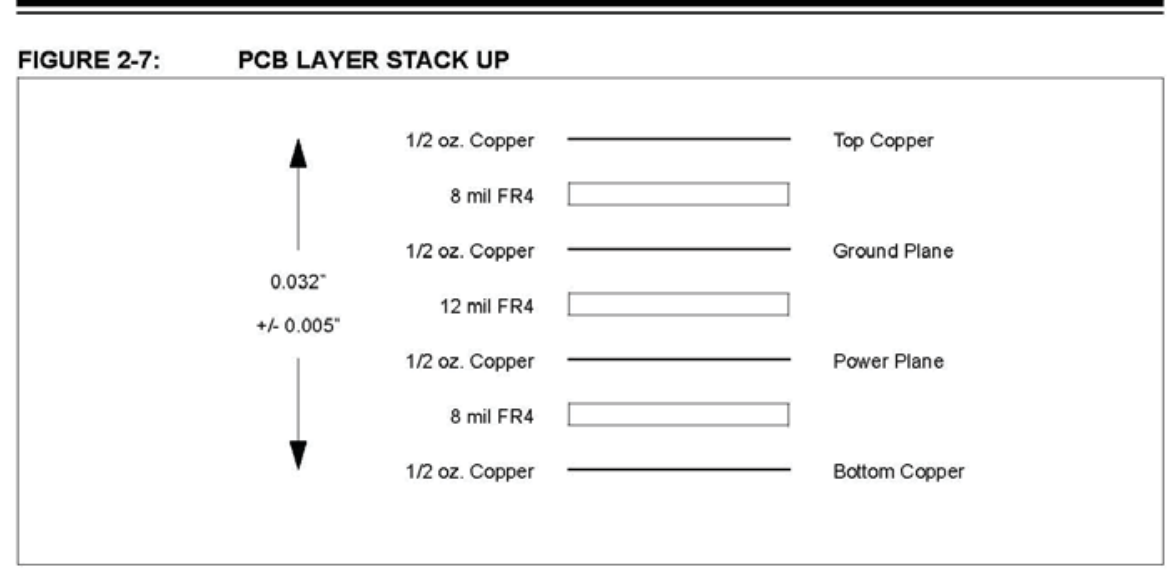




\section{MRF24J40MA}

\subsection{PCB Antenna}

The PCB antenna is fabricated on the top copper trace. Figure 2-8 shows the trace dimensions. The layers below the antenna have no copper traces. The ground and power planes under the components serve as a counterpoise to the PCB antenna. Additional ground plane on the host PCB will substantially enhance the performance of the module. For best performance, place the module on the host PCB following the recommendations in Section 1.2 "Mounting Details". The Printed Circuit Board (PCB) antenna was designed and simulated using Ansoft Designer ${ }^{(8)}$ and HFSS ${ }^{\mathrm{TM}}$ 3D full-wave solver software by Ansoft Corporation (mwwansoftcom). The design goal was to create a compact, low-cost antenna with the best radiation pattern. Figure 2-9 shows the simulation drawing and Figure 2-10 and Figure 2-11 show the $2 D$ and $3 D$ radiation patterns, respectively. As shown by the radiation patterns, the performance of the antenna is dependant upon the orientation of the module Figure 2-12 shows the impedance simulation and Figure $2-13$ shows the actual impedance measurement. The discrete matching circuitry matches the impedance of the antenna with the MRF24J40 transceiver IC.

\section{FIGURE 2-9: $\quad$ PCB ANTENNA SIMULATION DRAWING}

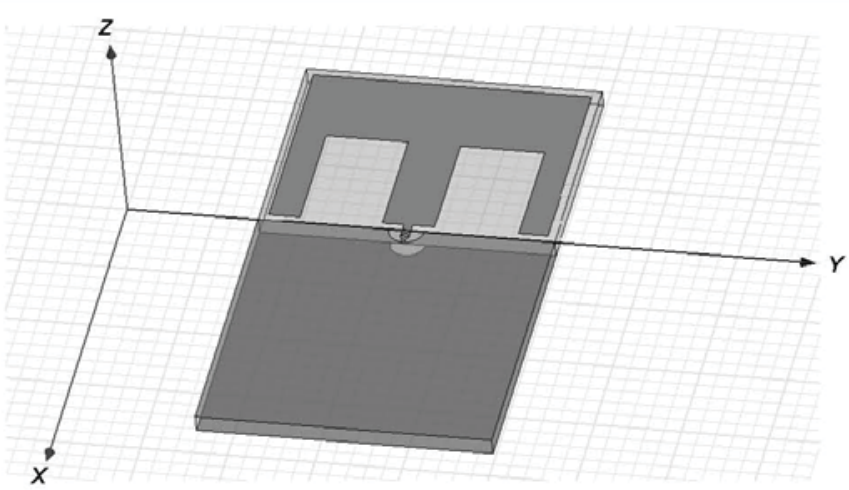


MRF24J40MA

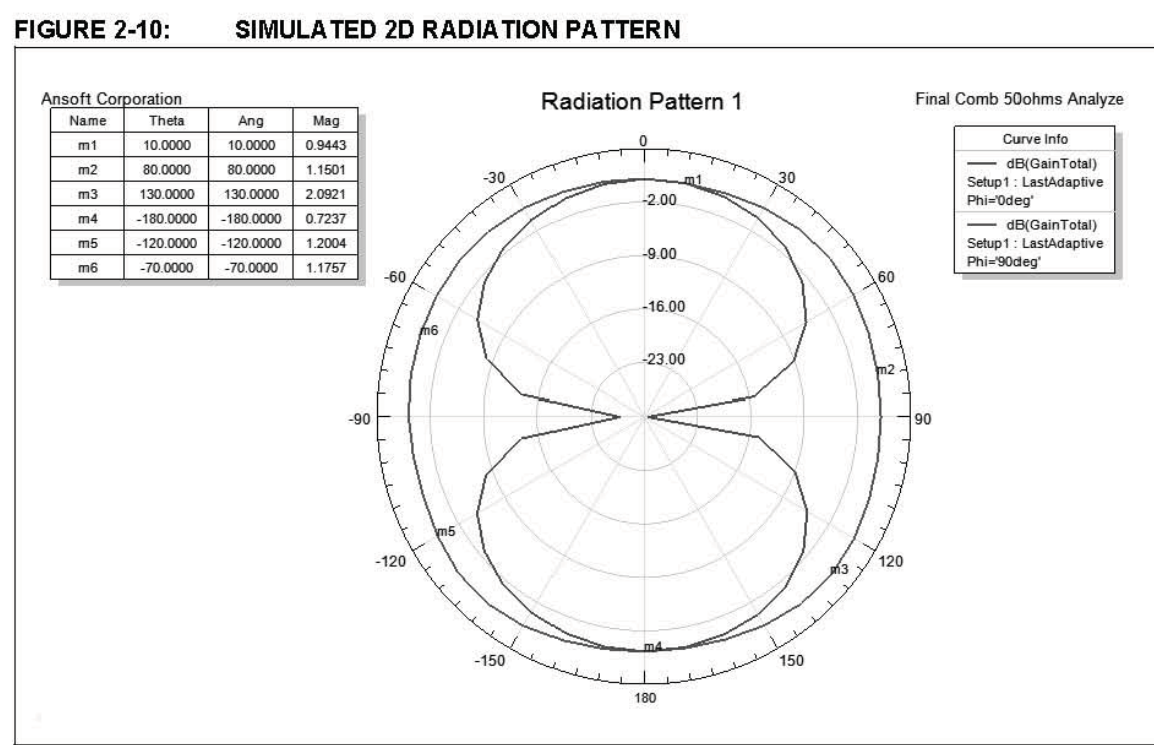

\section{FIGURE 2-11: SIMULATED 3D RADIATION PATTERN}

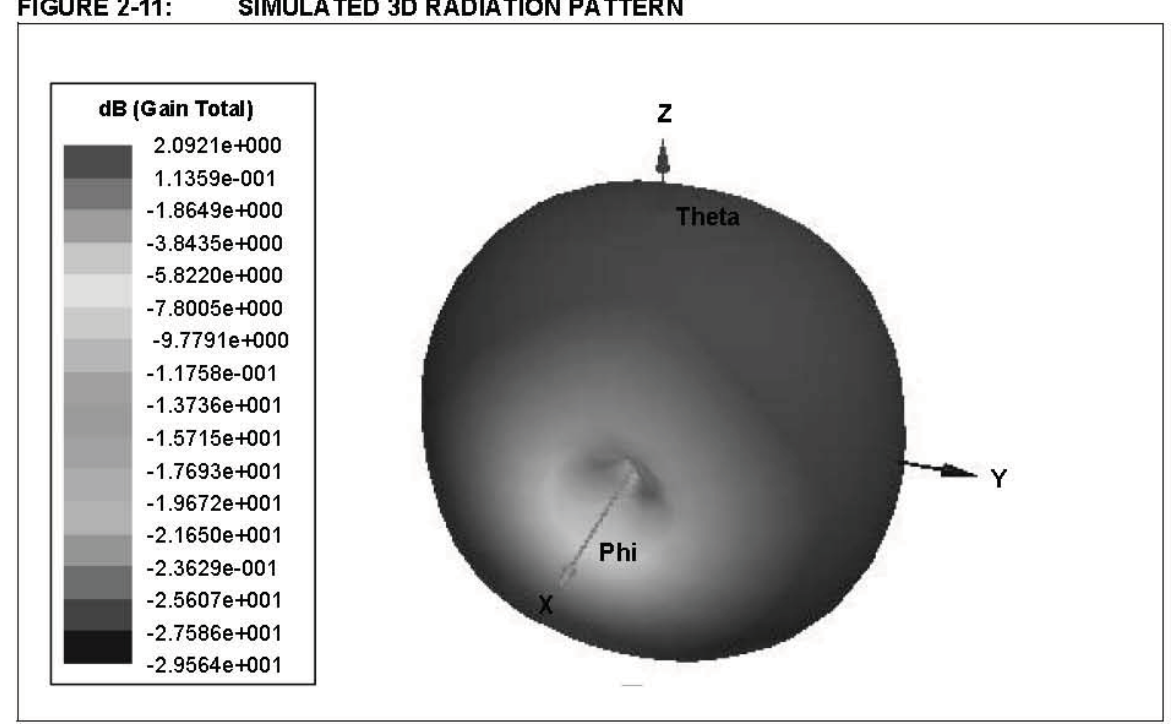


MRF24J40MA

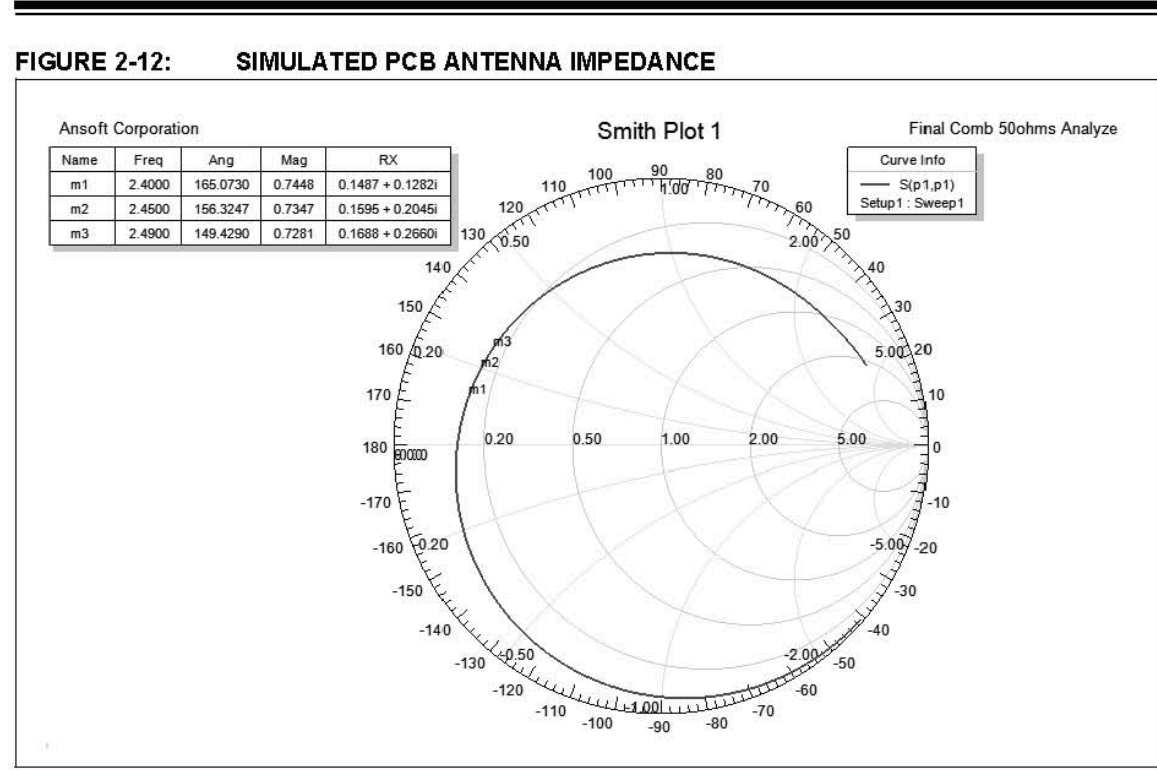

FIGURE 2-13: MEASURED PCB ANTENNA IMPEDANCE 


\subsection{REGULATORY APPROVAL}

The MRF24J40MA module has received regulatory approvals for modular devices in the United States Canada and European countries. Modular approva allows the end user to place the MRF24J40MA module inside a finished product and not require regulatory testing for an intentional radiator (RF transmitter), provided no changes or modifications are made to the module circuitry. Changes or modifications could void the user's authority to operate the equipment. The end user must comply with all of the instructions provided by the Grantee, which indicate installation and/or operating conditions necessary for compliance.

The integrator may still be responsible for testing the end product for any additional compliance requirements required with this module installed (for example: digital device emission, PC peripheral requirements, etc.) in the specific country that the end device will be marketed.

Annex F of the IEEE Std. 802.15.4 document has a good summary of regulatory requirements in various countries concerning IEEE Std. 802.15.4 devices. The standard can be downloaded from the IEEE Standards web page: http://standards. ieee. org/getieee802/802.15. htm

Refer to the specific country radio regulations for details on regulatory compliance.

\subsection{United States}

The MRF24J40MA has received Federal Communications Commission (FCC) CFR47 Telecommunications Part 15 Subpart C "Intentional Radiators" 15.247 and modular approval in accordance with FCC Public Notice DA 00-1407 Released: June 26, 2000, Part 15 Unlicensed Modular Transmitter Approval. The MRF24J40MA module can be integrated into a finished product without obtaining subsequent and separate FCC approvals.

The MRF24J40MA module has been labeled with its own FCC ID number, and if the FCC ID is not visible when the module is installed inside another device. then the outside of the finished product into which the module is installed must also display a label referring to the enclosed module. This exterior label can use wording such as the following:

\section{Contains Transmitter Module FCC ID OA3MRF24J40MA \\ -or- \\ Contains FCC ID: OA3MRF24J40MA}

This device complies with Part 15 of the FCC Rules. Operation is subject to the following two conditions: (1) this device may not cause harmful interference, and (2) this device must accept any interference received, including interference that may cause undesired operation.
The user's manual should include the following statement:

This equipment has been tested and found to comply with the limits for a Class B digital device, pursuant to part 15 of the FCC Rules. These limits are designed to provide reasonable protection against harmful interference in a residential installation. This equipment generates, uses and can radiate radio frequency energy, and if not installed and used in accordance with the instructions, may cause harmful interference to radio communications. However, there is no guarantee that interference will not occur in a particular installation. If this equipment does cause harmful interference to radio or television reception, which can be determined by turning the equipment of and on, the user is encouraged to try to correct the interference by one or more of the following measures:

- Reorient or relocate the receiving antenna.

- Increase the separation between the equipment and receiver

- Connect the equipment into an outlet on a circuit different from that to which the receiver is connected.

- Consult the dealer or an experienced radio/TV technician for help.

\subsubsection{RF EXPOSURE}

All transmitters regulated by FCC must comply with RF exposure requirements. OET Bulletin 65 "Evaluating Compliance with FCC Guidelines for Human Exposure to Radio Frequency Electromagnetic Fields" provides assistance in determining whether proposed or existing transmitting facilities, operations or devices comply with limits for human exposure to Radio Frequency (RF) fields adopted by the Federal Communications Commission (FCC). The bulletin offers guidelines and suggestions for evaluating compliance.

If appropriate, compliance with exposure guidelines for mobile and unlicensed devices can be accomplished by the use of warning labels and by providing users with information concerning minimum separation distances from transmitting structures and proper installation of antennas. 


\section{MRF24J40MA}

The following statement must be included as a CAUTION statement in manuals and OEM products to alert users of FCC RF Exposure compliance:

To satisfy FCC RF Exposure requirements for mobile and base station transmission devices, a separation distance of $20 \mathrm{~cm}$ or more should be maintained between the antenna of this device and persons during operation. To ensure compliance, operation at closer than this distance is not recommended.

The antenna(s) used for this transmitter must not be co-located or operating in conjunction with any other antenna or transmitter.

If the MRF24J40MA module is used in a portable application (antenna is less than $20 \mathrm{~cm}$ from persons during operation), the integrator is responsible for performing Specific Absorption Rate (SAR) testing in accordance with FCC rules 2.1091

\subsubsection{HELPFUL WEB SITES}

Federal Communications Commission (FCC) http://Wunw.foc.gov

\subsection{Canada}

The MRF24J40MA module has been certified for use in Canada under Industry Canada (IC) Radio Standards Specification (RSS) RSS-210 and RSS-Gen.

From Section 7.1.1, RSS-Gen, Issue 2, June 2007, Modular Transmitter Approval

Host devices which contain separately certified modules do not need to be recertified, provided that they meet the following conditions

a) The host device, as a stand-alone unit without any separately certified modules complies with all applicable Radio Standards Specifications.

b) The host device and all the separately certified modules it contains jointly meet the RF exposure compliance requirements of RSS-102, if applicable

c) The host device complies with the certification labeling requirements of each of the modules it contains.

Note: Compliance of a module in its final configuration is the responsibility of the applicant. A host device will not be considered certified if the instructions regarding antenna configuration provided in the original description, of one or more separately certified modules it contains, were not followed
From Section 5.2, RSS-Gen, Issue 2, June 2007, Equipment Labels:

All Category I radio equipment intended for use in Canada shall permanently display on each transmitter, receiver or inseparable combination thereof, the applicant's name (i.e., manufacturer's name, trade name or brand name), model number and certification number. This information shall be affixed in such a manner as to not be removable except by destruction or defacement. The size of the lettering shall be legible without the aid of magnification, but is not required to be larger than 8-point font size. If the device is too small to meet this condition, the information can be included in the user manual upon agreement with Industry Canada.

Label:

Contains IC: 7693A-24J40MA

From Section 7.1.6, RSS-Gen, Issue 2, June 2007, Digital Circuits:

If the device contains digital circuitry that is not directly associated with the radio transmitter, the device shall also have to comply with ICES-003, Class A or B as appropriate, except for ICES-003 labeling requirements. The test data obtained (for the ICES-003 tests) shall be kept by the manufacturer or importer whose name appears on the equipment label, and made available to Industry Canada on request, for as long as the model is being marketed in Canada.

\subsubsection{HELPFUL WEB SITES}

Industry Canada: http://umw.ic.gc.ca/ 
MRF24J40MA

\subsection{Europe}

The MRF24J40MA module has been certified for use in European countries. The following testing has been completed:

Test standard ETSI EN 300328 V1.7.1 (2006-10):

- Maximum Transmit Power

- Maximum EIRP Spectral Density

- Frequency Range

- Radiated Emissions

Test standards ETSI EN 301 489-1:2008 and ETSI EN 301 489-17:2008:

- Radiated Emissions

- Electro-Static Discharge

- Radiated RF Susceptibility

A helpful document that can be used as a starting point in understanding the use of Short Range Devices (SRD) in Europe is the European Radio Communications Committee (ERC) Recommendation 70-03 E, downloadable from the European Radio Communications Office (ERO): http://mww.ero.dk.
The end user is responsible for ensuring compliance with harmonized frequencies and labeling requirements for each country the end device is marketed and sold.

3.3.1

HELPFUL WEB SITES

Radio and Telecommunications Terminal Equipment (R\&TTE):

http://ec.europa.eu/enterprise/rtte/index_en.htm

European Conference of Postal and Telecommunications Administrations (CEPT): http://umw.cept.org/

European Telecommunications Standards Institute (ETSI): http://umw.etsi.org/

European Radio Communications Office (ERO) http://wnww.ero.dk 
MRF24J40MA

NOTES: 
MRF24J40MA

\subsection{ELECTRICAL CHARACTERISTICS}

TABLE 4-1: RECOMMENDED OPERATING CONDITIONS

\begin{tabular}{|l|c|c|c|c|}
\hline \multicolumn{1}{|c|}{ Parameters } & Min & Typ & Max & Units \\
\hline \hline Ambient Operating Temperature & -40 & - & +85 & ${ }^{\circ} \mathrm{C}$ \\
\hline $\begin{array}{l}\text { Supply Voltage for RF, Analog and } \\
\text { Digital Circuits }\end{array}$ & 2.4 & - & 3.6 & $\mathrm{~V}$ \\
\hline Supply Voltage for Digital I/O & 2.4 & 3.3 & 3.6 & $\mathrm{~V}$ \\
\hline Input High Voltage (VIH) & $0.5 \times$ VDD & - & VDD + 0.3 & V \\
\hline Input Low Voltage (VIL) & -0.3 & - & $0.2 \times$ VDD & V \\
\hline
\end{tabular}

TABLE 4-2: CURRENT CONSUMPTION

$\left(T A=25^{\circ} \mathrm{C}, V D D=3.3 \mathrm{~V}\right)$

\begin{tabular}{|l|l|c|c|c|c|}
\hline \multicolumn{1}{|c|}{ Chip Mode } & \multicolumn{1}{c|}{ Condition } & Min & Typ & Max & Units \\
\hline \hline Sleep & Sleep Clock Disabled & - & 2 & - & $\mu \mathrm{A}$ \\
\hline TX & At Maximum Output Power & - & 23 & - & $\mathrm{mA}$ \\
\hline RX & & - & 19 & - & $\mathrm{mA}$ \\
\hline
\end{tabular}

TABLE 4-3: RECEIVER AC CHARACTERISTICS

Typical values are at $T A=25^{\circ} \mathrm{C}, V D D=3.3 \mathrm{~V}$, LO Frequency $=2.445 \mathrm{GHz}$

\begin{tabular}{|l|l|c|c|c|c|}
\hline \multicolumn{1}{|c|}{ Parameters } & \multicolumn{1}{|c|}{ Condition } & Min & Typ & Max & Units \\
\hline \hline RF Input Frequency & $\begin{array}{l}\text { Compatible to } \\
\text { IEEE Std. 802.15.4 }{ }^{\mathrm{TM}}, 2003\end{array}$ & 2.405 & - & 2.480 & $\mathrm{GHz}$ \\
\hline RF Sensitivity & & - & -94 & - & $\mathrm{dBm}$ \\
\hline Maximum RF Input & & +5 & - & - & $\mathrm{dBm}$ \\
\hline LO Leakage & $\begin{array}{l}\text { Measured at Balun Matching } \\
\text { Network Input at } \\
\text { Frequency, 2.405-2.48 GHz }\end{array}$ & - & -60 & - & $\mathrm{dBm}$ \\
\hline $\begin{array}{l}\text { Input Return Loss } \\
\text { Externally Matched to 50 ohm Source } \\
\text { by a Balun Matching Network }\end{array}$ & -8 & -12 & - & $\mathrm{dB}$ \\
\hline $\begin{array}{l}\text { Noise Figure } \\
\text { (including matching) }\end{array}$ & & - & 8 & - & $\mathrm{dB}$ \\
\hline $\begin{array}{l}\text { Adjacent Channel } \\
\text { Rejection }\end{array}$ & @ +l-5 MHz & 30 & - & - & $\mathrm{dB}$ \\
\hline $\begin{array}{l}\text { Alternate Channel } \\
\text { Rejection }\end{array}$ & $@+/-10 \mathrm{MHz}$ & 40 & - & - & $\mathrm{dB}$ \\
\hline RSSI Range & & - & 50 & - & $\mathrm{dB}$ \\
\hline RSSI Error & & -5 & - & 5 & $\mathrm{~dB}$ \\
\hline
\end{tabular}


MRF24J40MA

TABLE 4-4: TRANSMITTER AC CHARACTERISTICS

Typical values are at $T A=25^{\circ} \mathrm{C}, V D D=3.3 \mathrm{~V}$, LO Frequency $=2.445 \mathrm{GHz}$

\begin{tabular}{|c|c|c|c|c|c|}
\hline Parameters & Condition & Min & Typ & $\operatorname{Max}$ & Units \\
\hline RF Carrier Frequency & & 2.405 & - & 2.480 & $\mathrm{GHz}$ \\
\hline $\begin{array}{l}\text { Maximum RF Output } \\
\text { Power }\end{array}$ & & - & 0 & - & $\mathrm{dBm}$ \\
\hline $\begin{array}{l}\text { RF Output Power } \\
\text { Control Range }\end{array}$ & & - & 36 & - & $\mathrm{dB}$ \\
\hline $\begin{array}{l}\text { TX Gain Control } \\
\text { Resolution }\end{array}$ & Programmed by Register & - & 1.25 & - & $\mathrm{dB}$ \\
\hline Carrier Suppression & & - & -30 & - & $\mathrm{dBc}$ \\
\hline $\begin{array}{l}\text { TX Spectrum Mask for } \\
\text { O-QPSK Signal }\end{array}$ & $\begin{array}{l}\text { Offset Frequency }>3.5 \mathrm{MHz} \text {, } \\
\text { at } 0 \mathrm{dBm} \text { Output Power }\end{array}$ & -33 & - & - & $\mathrm{dBm}$ \\
\hline TXEVM & & - & 15 & - & $\%$ \\
\hline
\end{tabular}


APPENDIX A: REVISION HISTORY

Revision A (June 2008)

Original data sheet for the MRF24J40MA device.

Revision B (November 2008)

Changed C17 to $1.0 \mathrm{pF}$ and removed CLKOUT signal. 
MRF24J40MA

NOTES: 
MRF24J40MA

\section{INDEX}

A

AC Characteristics
Receiver
Transmitter
Antenna Impedance
Measured PCB
Simulated PCB

B

Block Diagrams Microcontroller to MRF24J40MA Interface $\ldots . . . \ldots \ldots \ldots . . . . . .4$
MRF24J40MA

C

Circuit Description .

Customer Change Notification Service .................................24

Customer Notification Service $\ldots$

Customer Notification System .................................................2

Customer Support ............................................................24

E

Electrical Characteristics ..................................................19 Current Consumption Recommended Operating Conditions

Errata

European Radio Communications (ERC)

$\mathbf{F}$

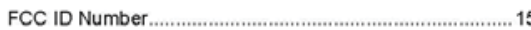

FCC RF Exposure Compliance

H

Helpful Web Sites

..16. 17

I

Interface Description .........................................................

Internet Address

M

MAC/Baseband Features.

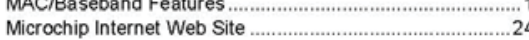

MiWi P2P.

MiWi Protocol

More Information

Customer Notification System....

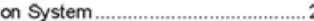

Errata

Mounting Details

MRF24J40 Data Sheet

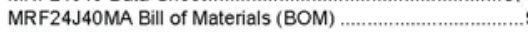

MRF24J40MA Schematic.

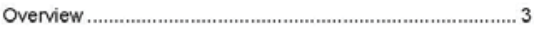

$\mathbf{P}$

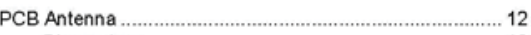
Dimensions ...................................................... 12

PCB Layers

Bottom Copper .................................................... 10

Layer 2 - Ground Plane ....................................... 10

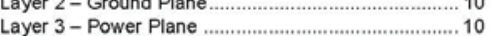

Layer 3 - Power Plane

Top Copper

Top Silk Screen ....................................................... 10

(PCB)

10

Radiation Pattern

$$
\text { 2D }
$$

3D

Reader Response

Regulatory Approva

Canada

United States

Revision History

RF Exposure ..............
RF/Analog Feature

$\mathbf{S}$

Serial I/O

SCK, SDI, SDO, $\overline{C S}$

Short Range Devices (SRD)

Specific Absorption Rate (SAR)

W

WuW Address...

WWW. On-Line Support

Z

ZigBee Protocol.

DS70329B-page 23

๑ 2008 Microchip Technology Inc 
MRF24J40MA

NOTES: 
MRF24J40MA

\section{THE MICROCHIP WEB SITE}

Microchip provides online support via our WMW site at unw.microchip.com. This web site is used as a means to make files and information easily available to customers. Accessible by using your favorite Internet browser, the web site contains the following information:

- Product Support - Data sheets and errata, application notes and sample programs, design resources, user's guides and hardware support documents, latest software releases and archived software

- General Technical Support - Frequently Asked Questions (FAQ), technical support requests. online discussion groups, Microchip consultant program member listing

- Business of Microchip - Product selector and ordering guides, latest Microchip press releases listing of seminars and events, listings of

Microchip sales offices, distributors and factory representatives

\section{CUSTOMER CHANGE NOTIFICATION} SERVICE

Microchip's customer notification service helps keep customers current on Microchip products. Subscribers will receive e-mail notification whenever there are changes, updates, revisions or errata related to a specified product family or development tool of interest

To register, access the Microchip web site at wnw.microchip.com, click on Customer Change Notification and follow the registration instructions.

\section{CUSTOMER SUPPORT}

Users of Microchip products can receive assistance through several channels:

- Distributor or Representative

- Local Sales Office

- Field Application Engineer (FAE)

- Technical Support

- Development Systems Information Line

Customers should contact their distributor, representative or field application engineer (FAE) for support. Local sales offices are also available to help customers. A listing of sales offices and locations is included in the back of this document

Technical support is available through the web site at: http://support.microchip.com 


\section{Appendix A-4 \\ Intellon INT6300 Technical Brief}

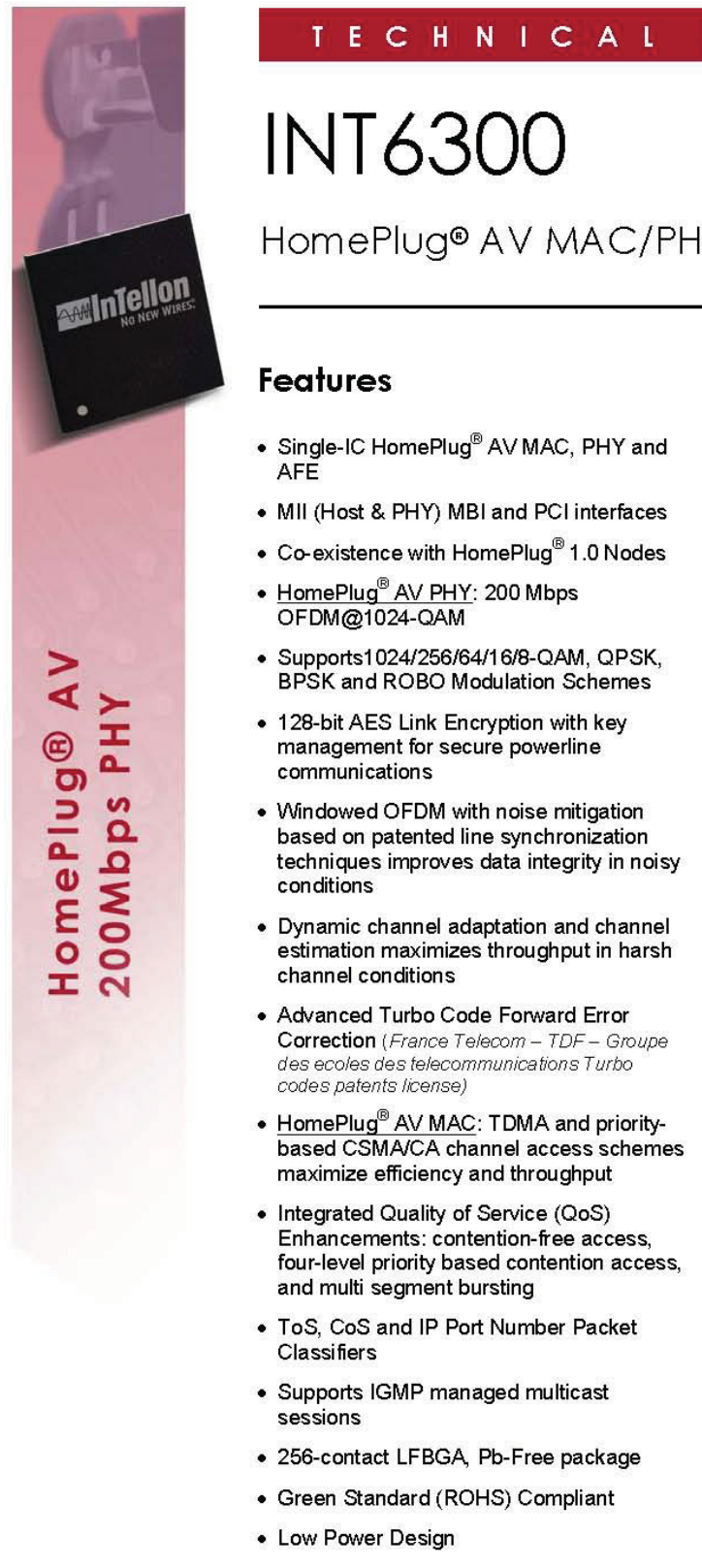

R O D U C T B R I E F

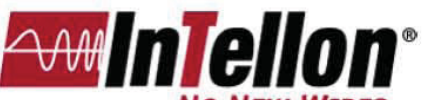

NO NEW WIRES。

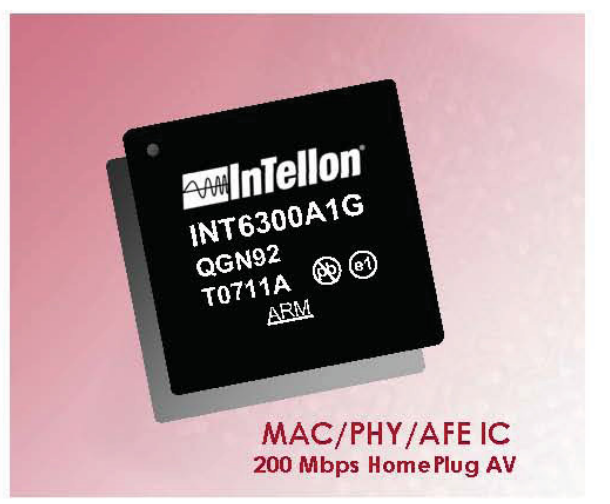

\section{Overview}

The INT6300 is Intellon's $2^{\text {nd }}$ generation HomePlug AV IC, offering higher performance and lower costs. Along with the AV MAC and PHY, the INT6300 includes the AFE. The integration of these blocks into one IC reduces total costs by reducing parts count, board space and assembly costs. Other internal improvements to the processing sections speed data flow and reduce latency.

The INT6300 IC is HomePlug AV standard-compliant delivering a $200 \mathrm{Mbps}$ physical layer bit rate, which supports multi-channel SD and HD digital video distribution, along with other data traffic, throughout the home.

The INT6300 provides Ethernet 802.3 network connectivity with extensions to support audio, video and data streams. The HPAV MAC manages segmentation and reassembly, network admission, and Quality of Service (QoS). The HPAV PHY transmits and receives Orthogonal Frequency Division Multiplexing (OFDM) symbols on the power line. 


\section{N T 63000 TECHN I CAL PRODUCT BR I EF}

\section{INT6300 Characteristics}

Physical

\begin{tabular}{|c|c|l|}
\hline Parameter & Value & \multicolumn{1}{c|}{ Description } \\
\hline Package Type & LFBGA-256. Pb-Free & $\begin{array}{l}256 \text { contact Low Profile and Fine Pitch Ball Grid Array, 16 X } \\
16 \text { array. 1mm ball pitch. MCM }\end{array}$ \\
\hline Dimensions & $17 \mathrm{~mm} \times 17 \mathrm{~mm}$ & Chip package dimensions \\
\hline Height & $1.61 \mathrm{~mm}$ & Maximum height above seating plane \\
\hline Operating Temp. Range & $0^{\circ} \mathrm{C}$ to $70^{\circ} \mathrm{C}$ & Safe package operating temperature range \\
\hline
\end{tabular}

Electrical

\begin{tabular}{|c|c|c|}
\hline Parameter & Value & Description \\
\hline Core Voltage $\left(V_{C \subset C E}\right)$ and PLL & 1.0 VDC. $-5 \%$ to $+10 \%$ & DC Core and PLL Voltage \\
\hline //O Voltage (VDDio) & 3.3 VDC, $+/-10 \%$ & DC I/O Voltage \\
\hline Analog Voltage $\left(V^{\prime} D_{A}\right)$ & $3.3 \mathrm{VDC},+/-10 \%$ & DC Analog Voltage \\
\hline ESD & $\begin{array}{c}1000 \mathrm{~V}(\mathrm{HBM}) \\
100 \mathrm{~V}(\mathrm{MM}) \\
250 \mathrm{~V}(\mathrm{CDM})\end{array}$ & ESD Immunity \\
\hline
\end{tabular}

Interfaces

\begin{tabular}{|c|l|}
\hline Type & \multicolumn{1}{c|}{ Description } \\
\hline MII (Ethernet) & Host or PHY. IEEE 802.3u Media Independent Interface \\
\hline PCI 2.2 Local Bus & Industry standard PCI 2.2 controller for video, voice, data and control \\
\hline Memory Bus Interface (MBI) & $\begin{array}{l}\text { 16-bit Memory Peripheral; compatible with Motorola MC68000 Slave Memory/Peripheral } \\
\text { Bus, ST Microelectronics Flash Memory/Peripheral Interface Bus (FMI) and Broadcom } \\
\text { External Bus Interface (EBI) }\end{array}$ \\
\hline SPI & $\begin{array}{l}\text { Serial Peripheral Interface to read/write INT6300 configuration and firmware image stored in } \\
\text { external flash memory and to configure the Analog Front End (AFE) analog transceiver }\end{array}$ \\
\hline SDRAM & $\begin{array}{l}\text { SDRAM memory controller operates at } 75 \mathrm{MHz}, 100 \mathrm{MHz}, 112.5 \mathrm{MHz} \text { or 150 MHz with 16- } \\
\text { bit or 32-bit data bus }\end{array}$ \\
\hline
\end{tabular}

Technologies

\begin{tabular}{|c|l|}
\hline Type & \multicolumn{1}{|c|}{ Description } \\
\hline Windowed OFDM & $\begin{array}{l}\text { Windowed Orthogonal Frequency Division Multiplexing - provides over 1.000 narrow-band } \\
\text { carriers each of which can be independently turned off (30-dB notch) or modulated up to } \\
\text { 1024-QAM for optimal performance }\end{array}$ \\
\hline TDMA Channel Access & $\begin{array}{l}\text { Time Division Multiple Access - a means of accommodating more than one service on the } \\
\text { channel by assigning time slots - useful for ensuring paramerterized Quality of Service } \\
\text { (QoS) for video streams }\end{array}$ \\
\hline CSMAVCA Channel Access & $\begin{array}{l}\text { Carrier-Sense Multiple Access/Collision Avoidance - a means of sensing the presence of a } \\
\text { carrier before transmission is attempted to avoid network collisions or contention }\end{array}$ \\
\hline
\end{tabular}

\section{Performance}

\begin{tabular}{|c|c|l|}
\hline Parameter & Value & \multicolumn{1}{c|}{ Description } \\
\hline Maximum PHY Rate & $200 \mathrm{Mbps}$ & Maximum PHY rate including payload and overhead \\
\hline Modulation & $1024 / 256 / 64 / 16 / 8$ QAM. & $\begin{array}{l}\text { Each OFDM sub carrier can be independently modulated } \\
\text { to optimize throughput for operating conditions }\end{array}$ \\
\hline Encryption & QPSK. BPSK, ROBO & Industry standard Advanced Encryption System \\
\hline
\end{tabular}




\section{N T 63000 TECH N I CAL PRODUCT B R I E F}

\section{INT6300 Architecture}

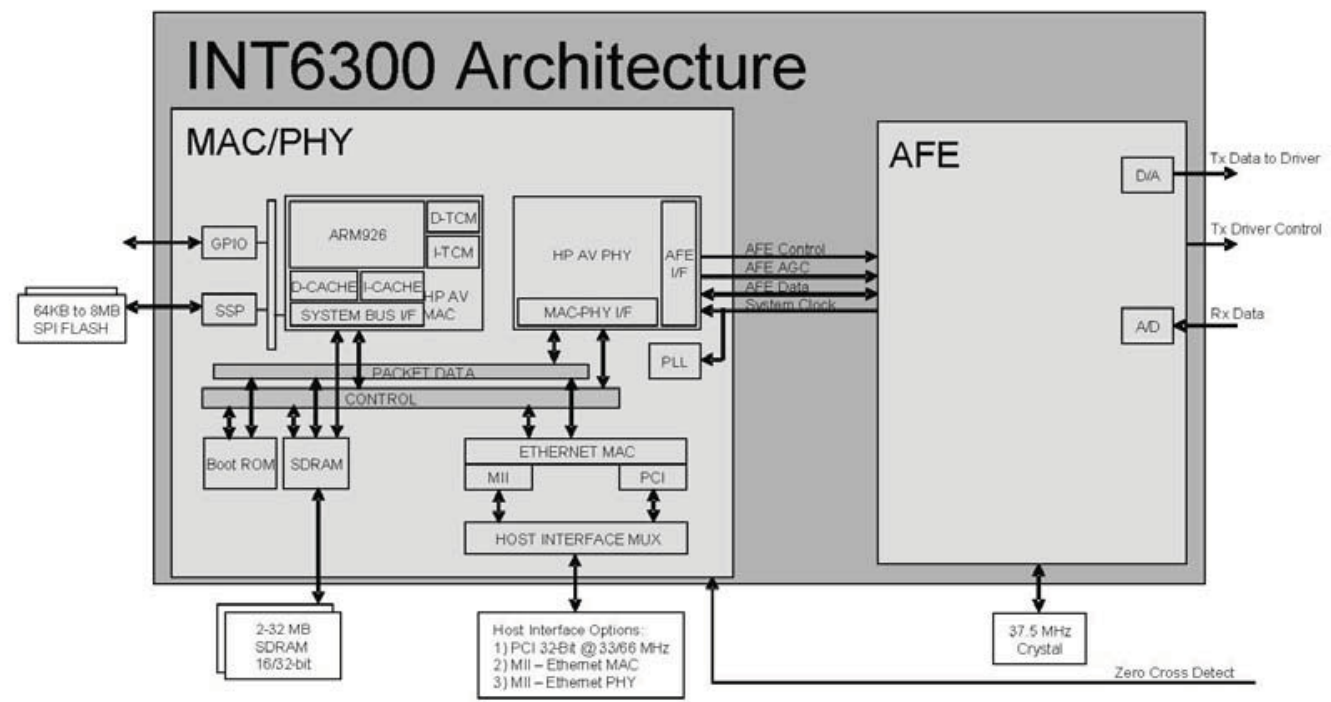

The INT6300 includes the HomePlug AV AFE - only a power amplifier and some external passive components are required to complete the analog interface. The AFE performs the required D/A, AVD, analog buffering and gain control functions. An external powerline coupler passes HomePlug AV OFDM signals to and from the powerline network while providing isolation from hazardous $A C$ power voltages.

Within the INT6300, the HomePlug-AV MAC function is carried out by firmware running on an embedded ARM926EJ-S CPU, supported by DMA hardware, two dedicated CRC engines, and a large off-chip SDRAM data-store. The HomePlugAV MAC firmware, running on the CPU, oversees operation of the integrated HomePlug AV PHY through an interface that carries control and status information in addition to transmit and receive data packets. General-purpose I/O pins are able to drive LEDs directly to indicate link status. An on-board PLL and built-in crystal oscillator driver permit generation of system clocks from a single external $37.5-\mathrm{MHz}$ crystal.

\section{Host Interface Applications}

The following sections describe various application scenarios in which the INT6300 is used, illustrating the use of the different interfaces. 


\section{N T 63000 TECHN I CAL PRODUCT BRIEF}

$\mathrm{PCl}$

The INT6300 PCl local bus controller, in the application shown in Figure 1, is used for video, voice and data streaming and for control and status exchange locally and over the power line. The PCI controller is a bridge between the INT6300 and an external $\mathrm{PCl}$ host.
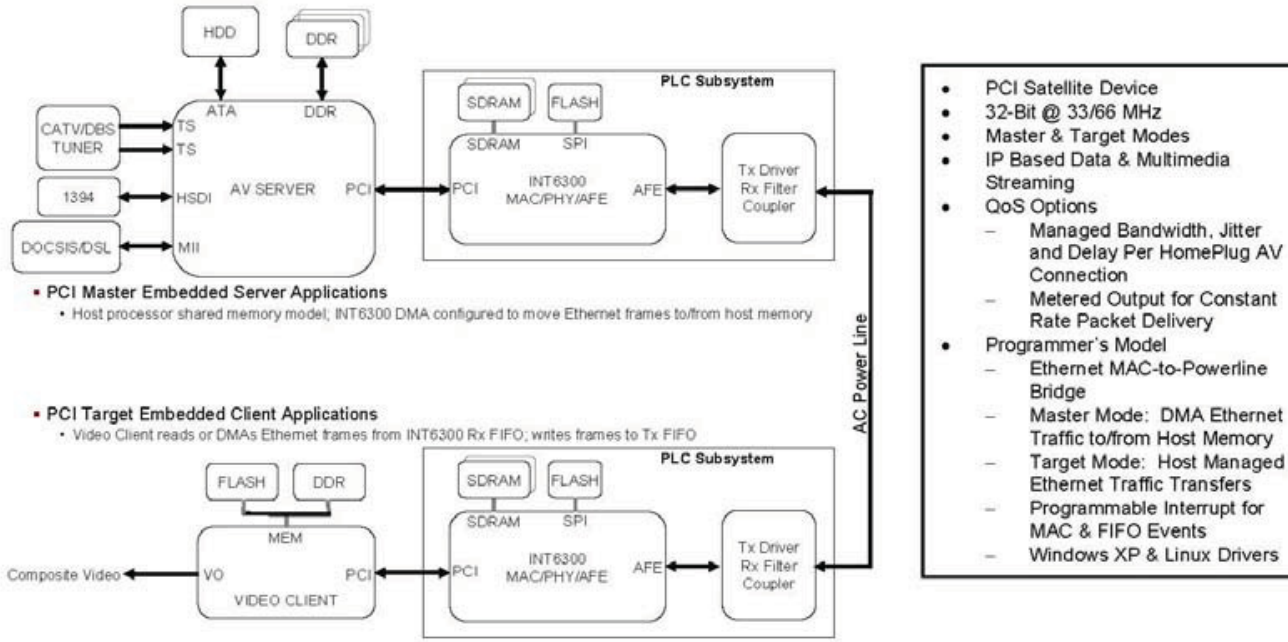

Figure 1 - PCI Local Bus Application

The PCl controller supports satellite operation only and may be configured as either a master or target single-function device. As a master device, it includes DMA channels for transmit and receive bus transactions. The PCI controller supports external bus master arbitration and bus parking.

\section{$\mathrm{MBI}$}

The Memory Bus Interface (MBI), illustrated in Figure 2, is used for video, voice and data streaming and for control and status exchange locally and over the power line. The MBI is designed to operate with three types of host devices:

1) Motorola M68000 Peripheral and Memory Bus compliant host devices configured for either asynchronous or synchronous operation

2) ST Microelectronics Flash Memory and Peripheral Bus (FMI) compliant host devices configured for either asynchronous or synchronous operation

3) Broadcom Corporation Enhanced Bus Interface (EBI) compliant host devices configured for M68000 compatible mode and either asynchronous or synchronous operation

The INT6300 MBI is a memory mapped slave controller and, therefore, does not require any bus control arbitration logic. The MBI slave controller operates as a networking peripheral with an embedded Ethernet MAC (EMAC) to emulate NIC functionality and to provide a common interface to application stacks designed for IP connectivity. 


\section{N T 63000 TECHN I CAL PRODUCT B R I E F}

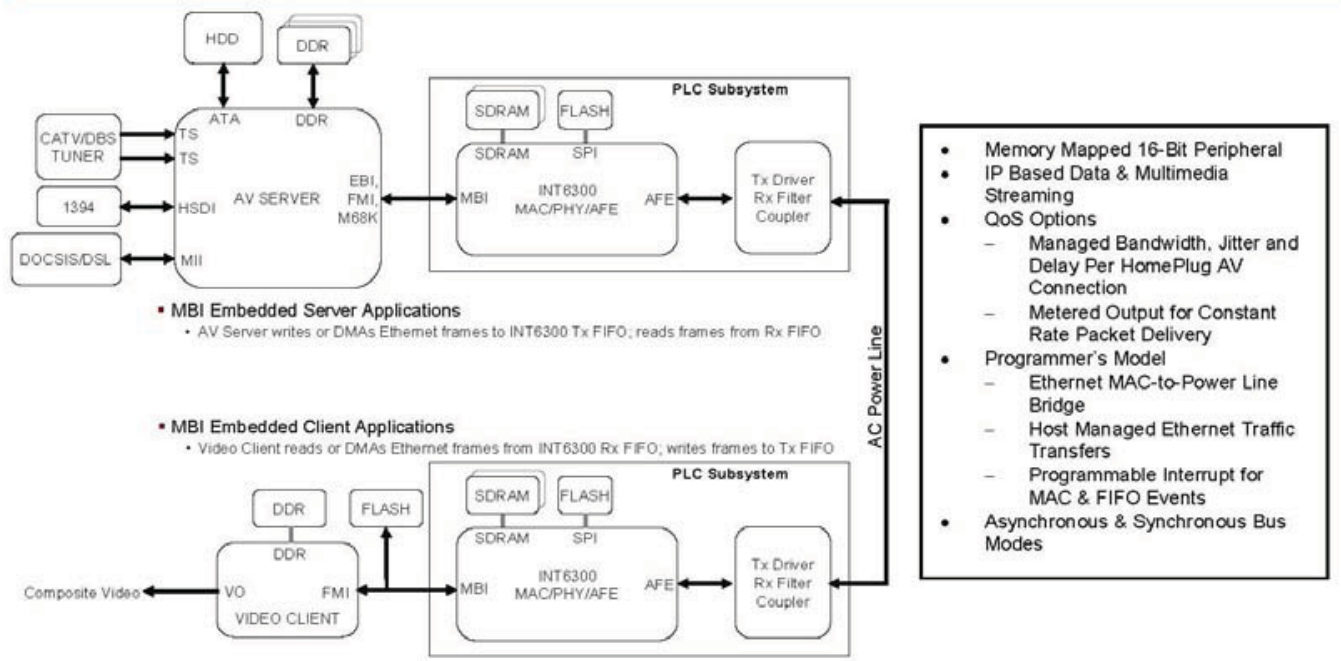

Figure 2 -Memory Bus Interface Application

\section{MII (Ethernet)}

The MII interface, illustrated in Figure 3, can be configured as either an Ethernet Host/DTE Controller or a Physical Medium Dependent (PMD or PHY) controller. MII is an industry standard, multi-vendor, interoperable interface between the Ethernet MAC and PHY sub-layers. It provides a simple connection between Ethernet PHY controllers and IEEE802.3 Ethernet MACs from a variety of sources.

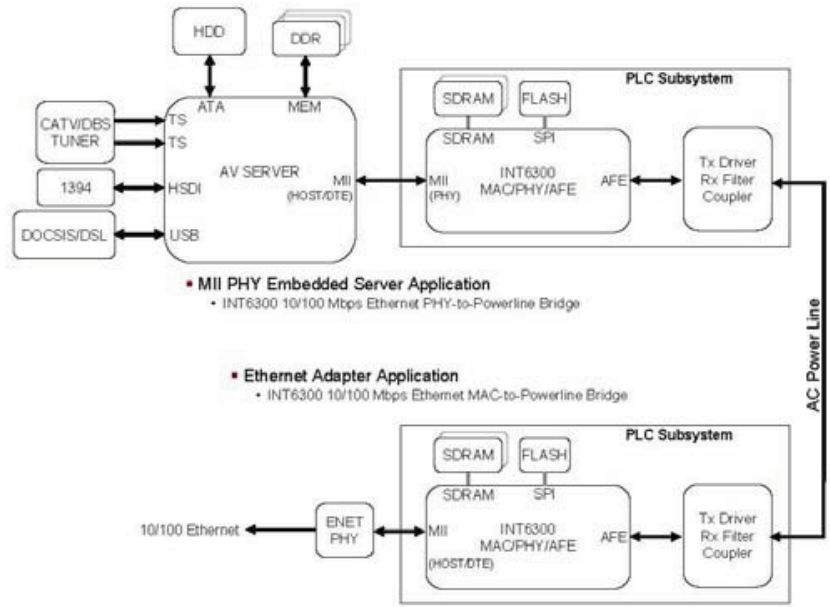

$$
\begin{aligned}
& \text { - IP Based Data \& Multimedia } \\
& \text { Streaming } \\
& \text { QoS Options } \\
& \text { - Managed Bandwidth. Jitter and } \\
& \text { Delay Per HomePlug AV } \\
& \text { Connection } \\
& \text { - Metered Output for Constant } \\
& \text { Rate Packet Delivery } \\
& \text { - Ethernet Host/DTE Mode } \\
& \text { 10/100 Ethernet MAC to Power } \\
& \text { Line Bridge } \\
& \text { - Full \& Half Duplex Flow Control } \\
& \text { Options } \\
& \text { Ethernet PHY Mode } \\
& \text { 10/100 Ethernet PHY to Power } \\
& \text { Line Bridge } \\
& \text { PHY Configuration Options } \\
& \text { - Auto-negotiation - On/Off } \\
& \text { - Speed - 10/100 } \\
& \text { - Duplex - Full/Half } \\
& \text { MD[4:3]-MD PHY Base } \\
& \text { Address } \\
& \text { Isolate - Disconnects MII } \\
& \text { Bus: MDIO \& MDC Active } \\
& \text { Status Bit Outputs: Link. } \\
& \text { Activity. Collision \& Speed }
\end{aligned}
$$

Figure 3 - MII Ethernet Application 


\section{NT 6300 TECHNICAL PRODUCT BRIEF}

Note that the AV Server, in Figure 3, utilizes the MII Host/DTE mode interface and is connected to the MII PHY mode interface of the INT6300. For the Ethernet adapter shown in Figure 3, the INT6300 uses MII Host/DTE to interface with the MII PHY (ENET PHY) on the Ethernet adapter. MII consists of separate 4-bit data paths for transmit and receive data along with carrier sense and collision detection. The Mll interface also provides a two-wire bi-directional serial

Management Data Interface (MDI). This interface provides access to the status and control registers in the Ethernet PHY logic. The MII and MDI pins are shared between the INT6300 MII Host/DTE and MII PHY interfaces.

Ordering Information: Part Number INT6300TR (lead-free on tape and reel) Lead-free is the only package option.

\begin{tabular}{|c|c|c|c|c|}
\hline 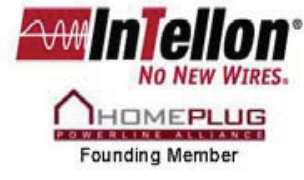 & $\begin{array}{l}\text { Corporate } \\
\text { Headquarters } \\
5955 T \text {.G. Lee Blvd. } \\
\text { Silte 600 } \\
\text { Orlando, FL } 32822 \\
(407) 428-2800 \\
(407) 428-2850 \text { (Fax) }\end{array}$ & $\begin{array}{l}\text { Ocala Office } \\
5100 \text { West Silver Springs } \\
\text { Blvd. } \\
\text { Ocala, FL } 34482 \\
\text { (352) 237-7416 } \\
\text { (352) 237-7616 (Fax) }\end{array}$ & $\begin{array}{l}\text { San Jose Office } \\
1731 \text { Technology Drive } \\
\text { Suite 560 } \\
\text { San Jose, CA } 95110 \\
\text { (408) 501-0320 } \\
\text { (408) 501-0323 (Fax) }\end{array}$ & $\begin{array}{l}\text { Toronto Office } \\
\text { 144 Front Street West, } \\
\text { Suite } 385 \\
\text { Toronto, Ontario M5J 2L7 } \\
\text { (416) 217-0451 } \\
\text { (416) 217-0459 (Fax) }\end{array}$ \\
\hline \multicolumn{5}{|c|}{ 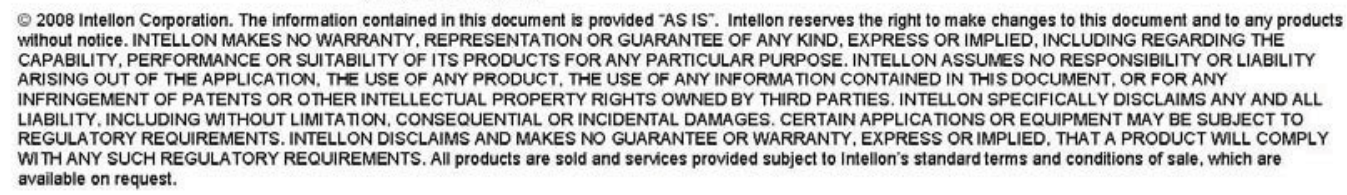 } \\
\hline alon and No New Wires are registere & & & & . \\
\hline
\end{tabular}




\section{Appendix A-5 Yitran IT700 Technical Brief}

IT700 IC - FEATURES and BENEFITS

The IT700 is a highly integrated System-on-a-Chip (SoC) Powerline Communication (PLC) IC that incorporates Yitran's extremely robust Physical Layer ( $\underline{\mathrm{PHY}})$, high-performance Data Link Layer ( $\underline{\mathrm{DLL}})$ and Network (Y-Net) protocol. IT700 also features a 8051 Micro with 256 KB Flash for protocol stack and application implementation as well as 24 general purpose I/Os.

The IT700 PLC modem core uses Yitran's patented Differential Code Shift Keying (DCSK) advanced spread spectrum modulation technique for extremely robust communication with data rates up to $7.5 \mathrm{Kbps}$. The device also utilizes several other mechanisms for enhanced communication robustness, such as a patented forward short-block soft-decoding error-correction algorithm and special synchronization algorithms.

The IT700 IC complies with worldwide regulations (FCC part 15, ARIB and CENELEC bands), is designed for HomePlug Command \& Control. IT700 is an ideal solution for a variety of command and control PLC applications.

The IT700 is available in two versions:

- The Protocol Controller Architecture version includes Yitran's Y-Net network protocol stack. A UART interface and simple command language provide seamless connection to an external Host controller and simplify application development. In this version, no access to the microcontroller's resources is provided.

- The Open Solution Architecture version allows utilization of the IT700 microcontroller's peripheral functions such as timers, interrupts, communication interfaces, A/D, spare memory resources and general-purpose I/Os to implement the application code, thereby eliminating the requirement for an external host controller. An Application Programming Interface (API) will enable easy integration of the application code with Yitran's code.

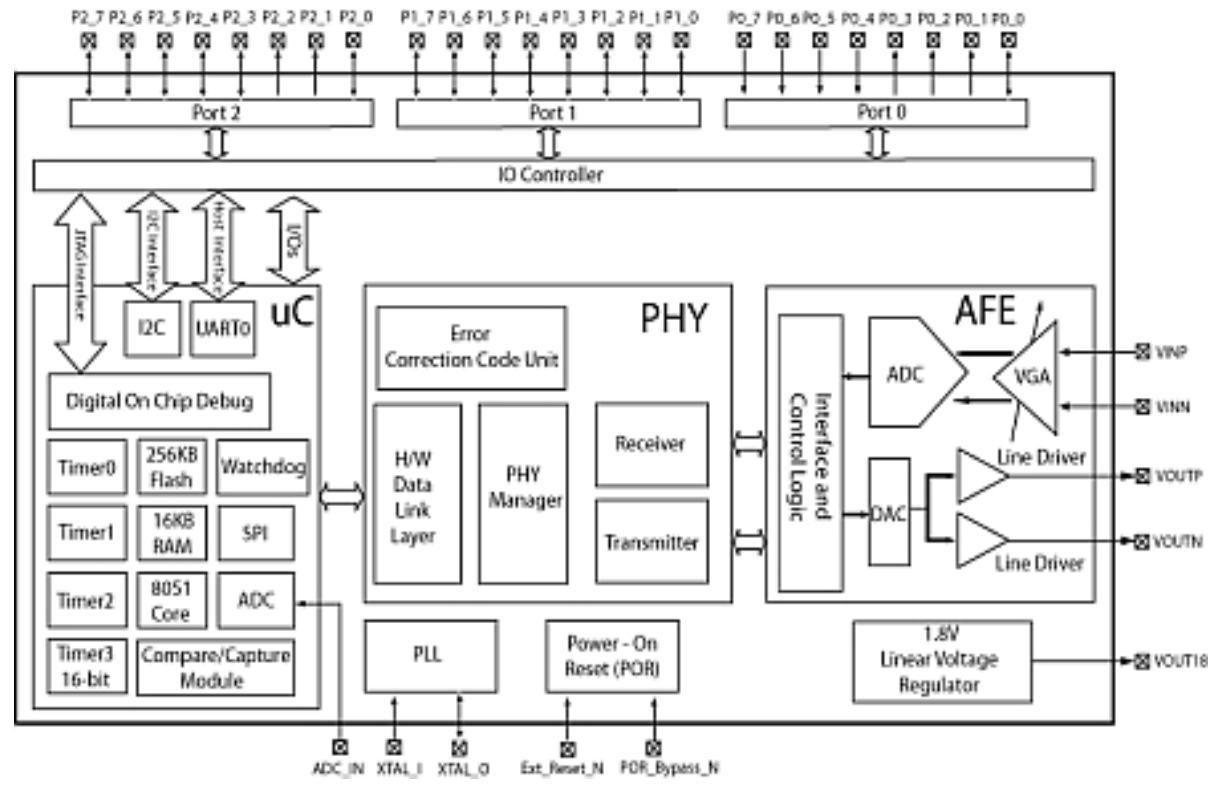




\section{Appendix A-6 Maxim 2990/2991 Data Sheets}

\section{General Description}

The MAX2990 power line communication (PLC) baseband modem delivers a cost-effective, reliable, halfduplex asynchronous data communication over $A C$ power lines at speeds up to $100 \mathrm{kbps}$. The MAX2990 is a highly integrated system-on-chip (SoC) that combines the physical (PHY) and media access control (MAC) layers using Maxim's 16-bit MAXQ microcontroller core. The MAX2990 utilizes OFDM modulation techniques to enable robust data communication using the same electrical network that supplies power to all other devices on the network.

The MAX2990 includes the MAXQ microcontroller core. The MAXQ is a 16-bit RISC microcontroller with $32 \mathrm{kB}$ flash memory, $5.12 \mathrm{kB}$ of ROM and 8kB SRAM, of which $4 \mathrm{kB}$ that can be simultaneously accessed by the MCU and the PHY. The MAX2990 is integrated with modules for serial communication (SPITM, I2C, UART) and a real-time clock (RTC) for time stamping, in addition to standard blocks such as timers, GPIO, and external interrupts.

The MAX2990 transceiver is based on an orthogonal frequency division multiplexing (OFDM) technique that allows robust data transmission over poor channel conditions specifically for environments with impulsive noise. OFDM with binary phase shift key (BPSK) and forward error correcting (FEC) blocks are used because of their inherent adaptability in the presence of frequency selective channels without the use of equalizers, resilience to jammer signals, robust communications in the presence of group delay spread, and robustness to impulsive noise. The MAX2990 features jammer cancellation that removes constant sinusoida interference signals for FCC and ARIB bands. Privacy is provided by DES encryption.

The MAX2990 is available in a 64-pin LQFP package and is specified over the $-40^{\circ} \mathrm{C}$ to $+85^{\circ} \mathrm{C}$ extended temperature range.

\section{Applications}

Automatic Meter Reading Sensor Control and Data Home Automation Acquisition

Heating Ventilation and Air Conditioning (HVAC) Building Automation Industrial Automation Lighting Control Remote Monitoring and Control

Voice-Over-Powerline Security Systems/Keyless Entry
Features

- Combines the Physical Layer (PHY) and Media Access Controller (MAC)

- Integrated Microcontroller with 32kB Password Protected Flash Memory and 8kB SRAM

- Maximum Effective Data Rate in Normal Mode $32 \mathrm{kbps}$ at $10 \mathrm{kHz}$ to $95 \mathrm{kHz}$ and $100 \mathrm{kbps}$ at $10 \mathrm{kHz}$ to $490 \mathrm{kHz}$

- Complies with CENELEC A (10kHz to $95 \mathrm{kHz})$ CENELEC B $(95 \mathrm{kHz}$ to $120 \mathrm{kHz})$ CENELEC C (120kHz to $140 \mathrm{kHz})$ FCC $(10 \mathrm{kHz}$ to $490 \mathrm{kHz})$ ARIB (10kHz to $450 \mathrm{kHz}$ )

- Includes Forward Error Correction (FEC) Mechanism and CRC16

- Includes Fast DES Engine as the Encryption/Decryption Coprocessor and CRC32

- Jammer Cancellation for FCC and ARIB

- User-Configured Start and End Operating Frequency

- Carrier Sense Multiple Access/Collision Avoidance (CSMA/CA) Channel Access Arbitration

- Automatic Repeat Request (ARQ) to Enhance Error Detection and Improve Data Reliability

- Supports SPI, I2C, and UART Interfaces

- Real-Time Clock (RTC)

- PWM Counters

- Built-In Test Mode Engine for Identifying Channel Conditions
Ordering Information

\begin{tabular}{|c|c|c|}
\hline PART & TEMP RANGE & PIN-PACKAGE \\
\hline MAX2990ECB+ & $-40^{\circ} \mathrm{C}$ to $+85^{\circ} \mathrm{C}$ & 64 LQFP \\
\hline
\end{tabular}

Pin Configuration appears at end of data sheet.

Maxia Maxim Integrated Products 1

For pricing, delivery, and ordering information, please contact Maxim Direct at 1-888-629-4642, or visit Maxim's website at www.maxim-ic.com. 


\section{ABRIDGED DATA SHEET}

\section{0kHz to 490kHz OFDM-Based}

Power Line Communication Modem

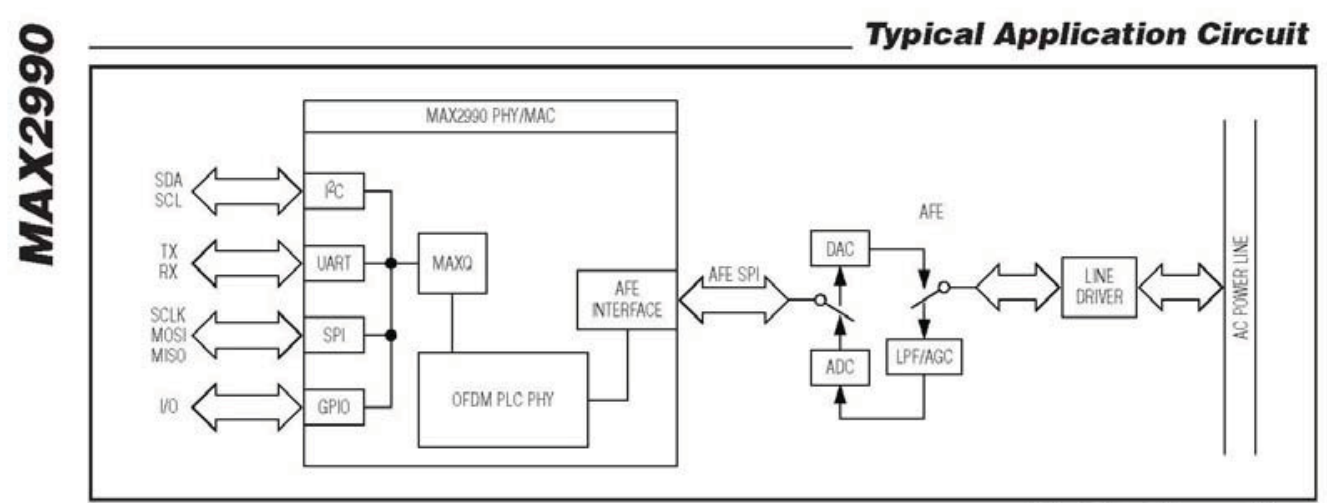

Pin Configuration

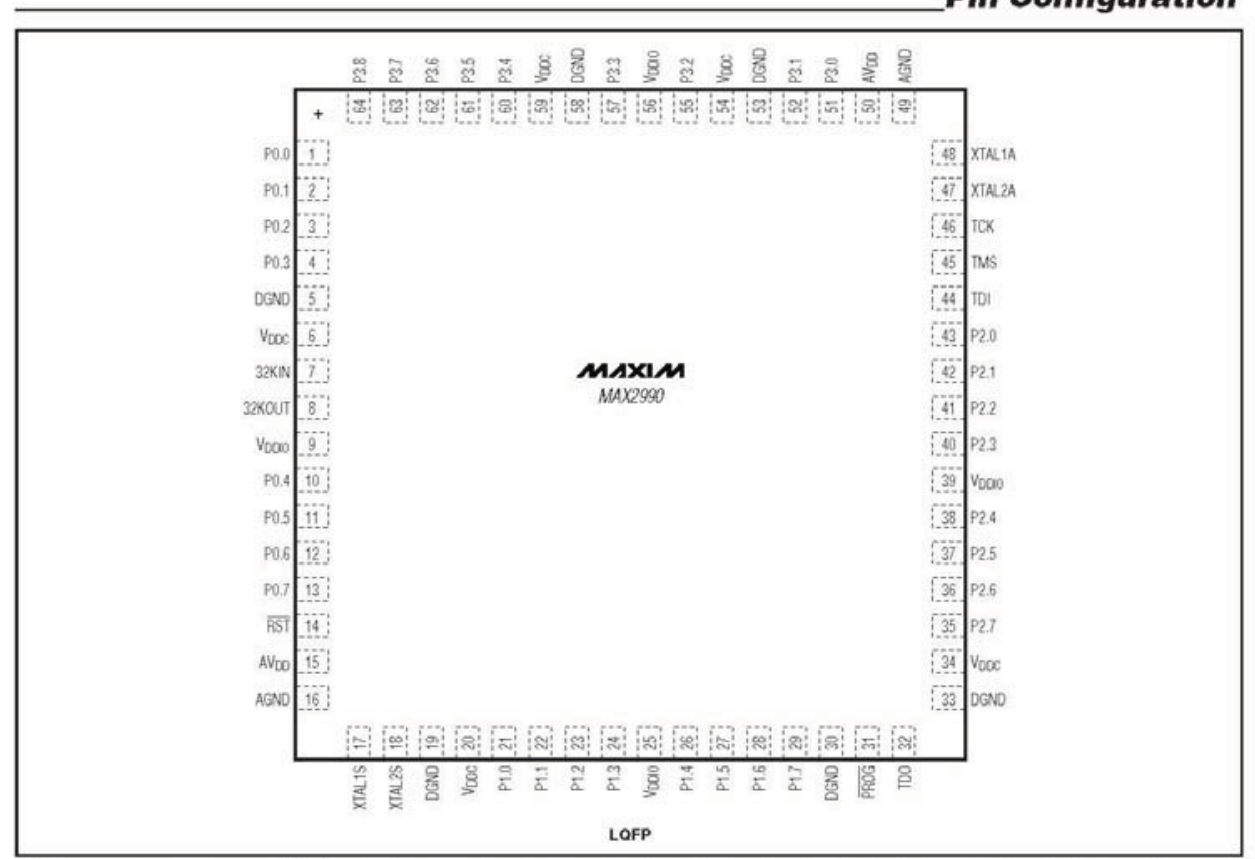

Maxim cannot assume responsibility for use of any circuitry other than circuitry entirely embodied in a Maxim product. No circult patent licenses are implied. Maxim reserves the right to change the circuitry and specifications without notice at any time.

24

Maxim Integrated Products, 120 San Gabriel Drive, Sunnyvale, CA 94086 408-737.7600

(6) 2008 Maxim Integrated Products

MAXIM is a registered trademark of Maxim Integrated Products, Inc. 
General Descriptlon

The MAX2901 powerline communication anabg frontend (AFE) is a state-of-the-art integrated circuit that delivers high integration and superb performance, whils reducing the total system cost. The MAX2991 is the first AFE specifically designed for OFDM (orthogonal frequency division multiplexing) modulated signal transmission over power lines. Operating in the $10 \mathrm{HHz}$ to $490 \mathrm{kHz}$ band, the programmable filters allow compliance with CENELEC, FOC, and ARIB standards using the same device.

The MAX2991 transceiver provides two main paths: transmit $(\mathrm{Tx})$ peth and receive $(\mathrm{Rx})$ path. The transmit path injects an OFDM modulated signal into the $A C$ or $\mathrm{DC}$ line. The transmit path is composed of a digital IIR filter, DAC (digital-to-analog converter), followed by a lowpass filter, and a pre-line diver. The receiver path is for the signal enhancement, filtering, and digitization of the received signal. The receiver is composed of a lowpass and a highpess filter, a wo-stage automatic gain control (AGC), and an ADC (analog-to-digital corverter). The integrated AGC maximizes the dynamic range of the signal up to $60 \mathrm{~dB}$, whils the lowpess filter removes any out-of band noise, and selects the desired frequency band. The ADC corverts the enhanced and amplified input signal to a digital format An integrated offeet cancellation locp minimizes the $\mathrm{DC}$ off set.

The MAX2991, abng with the MAX2990 PLC beseband modem, delivers the most cost-effective data communication solution over power line networks in the market. The MAX2991 is specified over the $-40^{\circ} \mathrm{C}$ to $+85^{\circ} \mathrm{C}$ temperature range and is available in a 48-pin LQFP package.

Features

- Optimized to Operate with the MAX2990 PLC Bareband

- Integrated Band Seleot Filter, AGG, and a 10-Bit ADC for Rx Path

- Integrated Wave-shaping Filter, Programmable Pre-Driver Gain, and a 10-Bit DAC for Tx Path

- Variable sampling Rate Up to 1.2Msps

- Built in 60dB Dynamio Range AGC and DC Offset Cancellation

- Programmable Filters Operate in the CENELEC, $F C C$, and ARAB Frequenoy Bands

- Single 3.3V Power Supply

+ 70mA Typioal Supply Current (Half-Duplex Moda)

- Extended Operating Temperature Range

Appllcations

Automatic Meter Reading

Home Automation

Hseting Ventilation and A r Conditioning (HVAC)

Building Automation

Industrial Automation

Lighting Control

Sensor Control and Deta Acquisition (SCADA)

Remote Monitoring and Control

Security Systems/Keyless Enty

Smart Grid

Ordering Information

\begin{tabular}{|ccc|}
\hline PART & TEMP RANGE & PIN-PACKAGE \\
\hline MAX2991ECM+ & $-40^{\circ} \mathrm{C}$ to $+65^{\circ} \mathrm{C}$ & 48 LQFP \\
\hline
\end{tabular}

+Denotes a lead(Pb)-traesRoHs-compliant package.

Typlcal Appllcation CIrcult

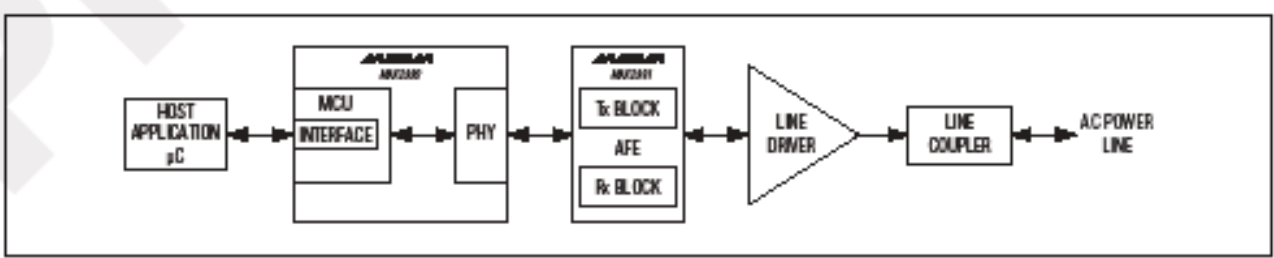

Maxim

Maxim Integr ated Products 1

For pricing, delivery, and ordering information, please contact Maxim Direct at 1-888-629-4642, or visit Maxim's website at www.maxim-ic.com. 


\section{Appendix A-7 Functional Test Setup Drawings}

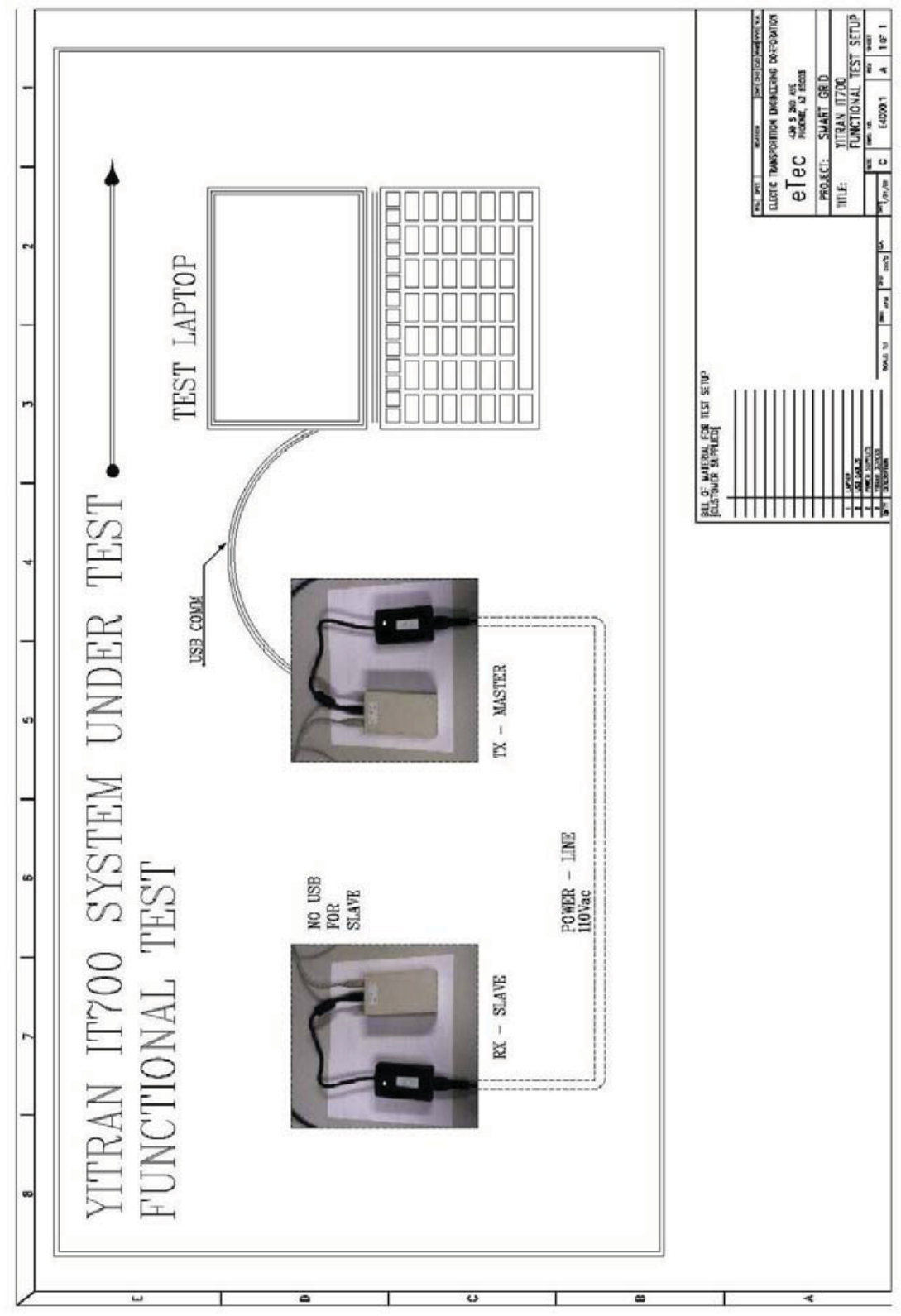




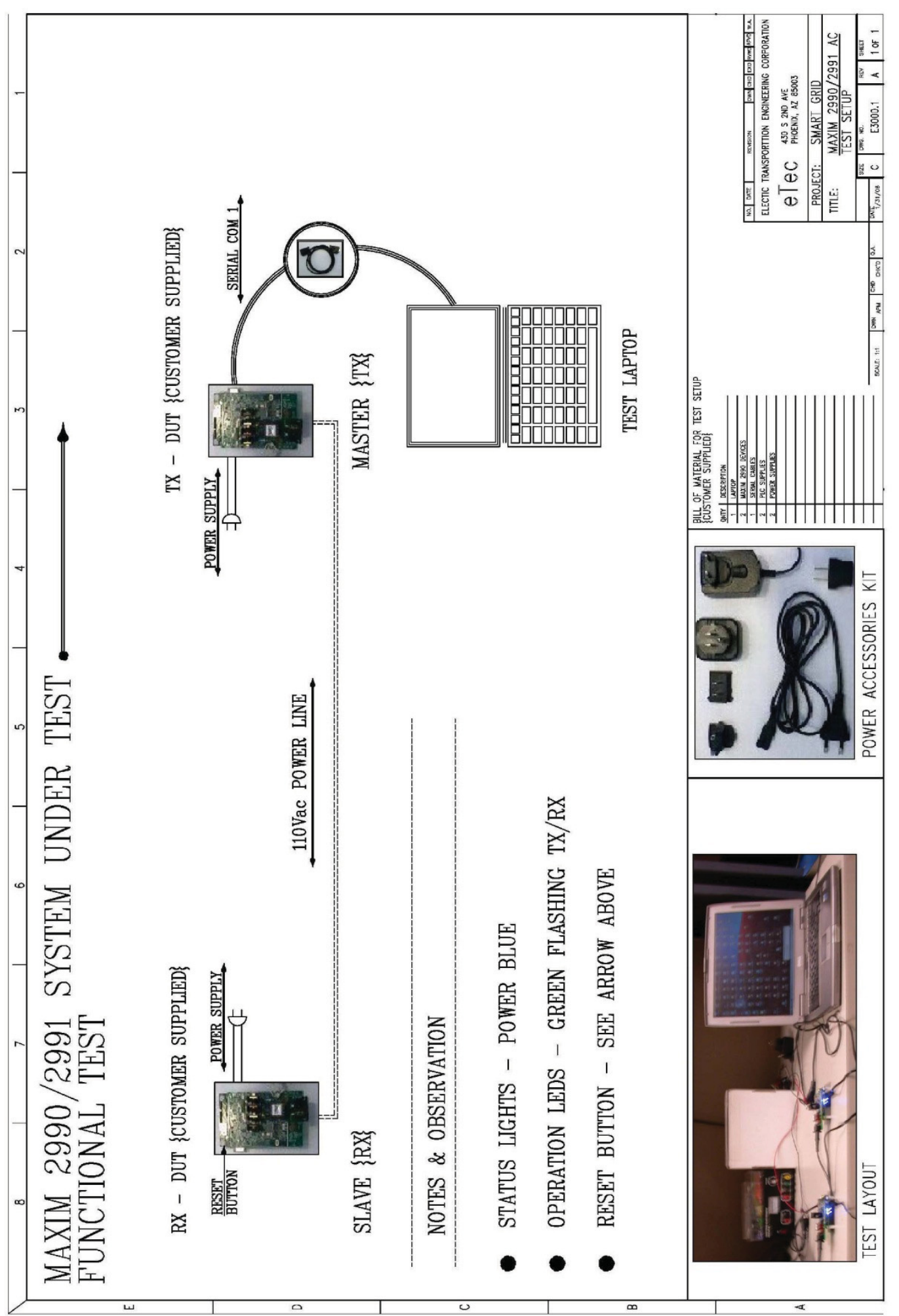




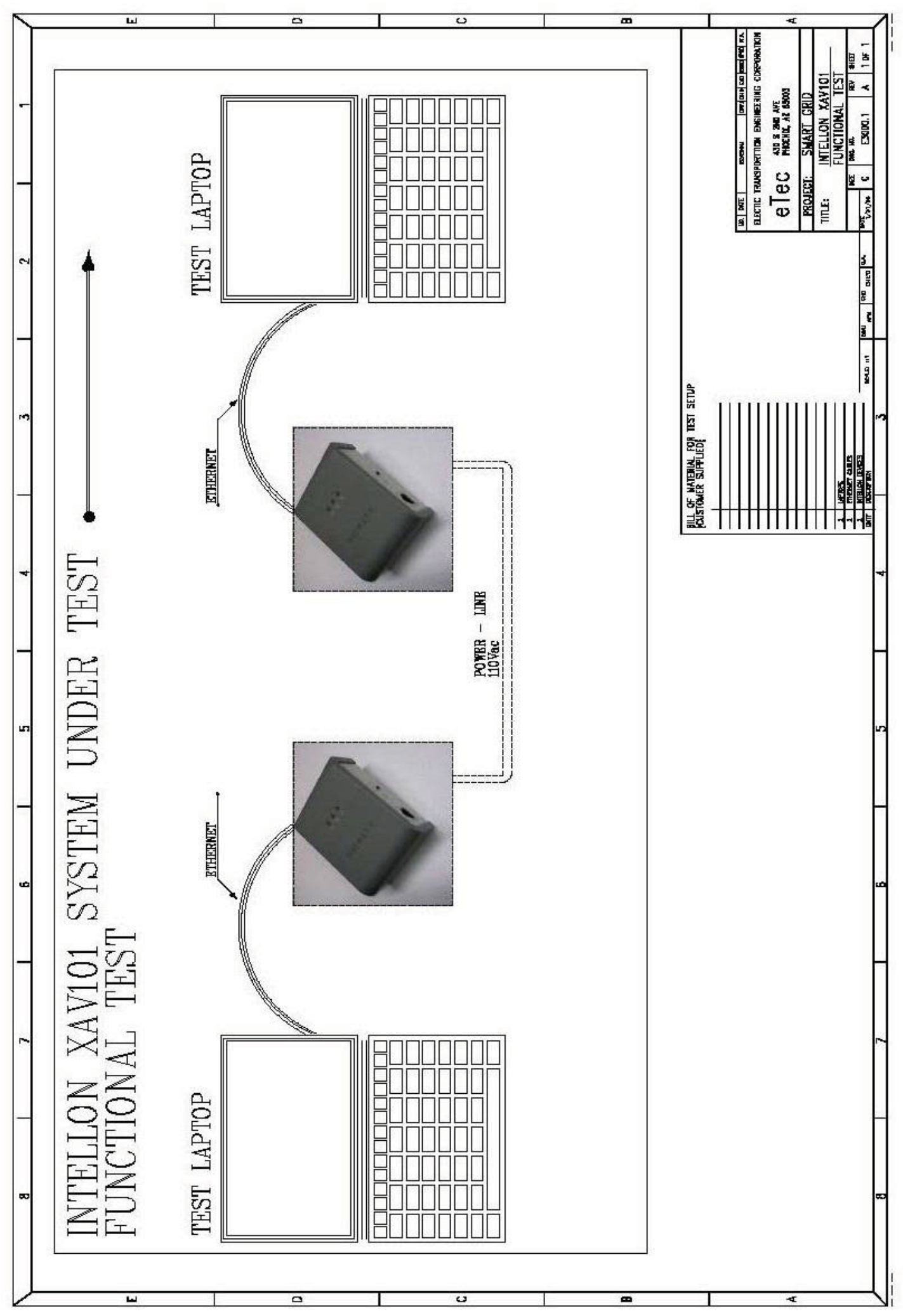




\title{
Appendix A-8 Functional Test Setup Instructions
}

\author{
EMC Test Setup \& Operation for PLC Communication \\ Released $7-28-2009$ \\ Document Rev 1.0
}

Preliminary: The EMC Test setup and operation consists of four test configurations, and a test operation for each one. Test configurations are broken down as follows:

- Vitron AC Setup \& Operation Phase I:

- Vitron DC Setup \& Operation Phase II:

- Interlon: Ethernet over Powerline Phase III:

- MAXIM PLC 2990 AC 110 \& 240 Hot Line:

- MAXIM PLC 2990 12V DC Setup \& Operation Phase V:

Instruction: Each phase consists of instructions and references to drawings, manuals and screen shots as they apply to the test operation and setup.

Phase I: Vitron AC Setup and Test:

Required Material: The following materials will be needed for setting up Phase I

- Two Vitron Units labeled 1,2

- One USB Cable

- Two AC Power Supplies for the Vitron Units.

- One Laptop Computer with Vitron Software

Procedure: Apply the following steps in setting up and operating the Vitron units in AC mode using the following steps and accompanying diagrams and drawings.

\section{Hardware Setup}

Step 1: Using Figure 1.0 located on page 1 of 2 on the drawing titled, "EMC_Test Layout" as a reference connect Vitron 1 to the test laptop using a USB cable.

Step 2: Connect Vitron 1 to its power adapter and plug the power adapter into a receptacle.

Step 3: Connect Vitron 2 to its power adapter and plug it into a separate receptacle away from where Vitron 1 is connected. Do not plug both Vitron 1 and Vitron 2 into the same receptacle. 
EMC Test Setup \& Operation for PLC Communication

Released $7-28$ - 2009

Document Rev 1.0

Observes Vitron 2 does not have a USB cable connected to the laptop. This is a deliberate action given the interest in measuring PLC communication exclusively.

This completes setting up the hardware for this Phase.

Phase I: Vitron AC Setup and Test Continued:

Step 4: Turn on the test laptop and turn on Vitron 1. Select Vitron Icon on the desktop. This starts the Test software specially made to communicate with Vitron. The following screen shot should display.

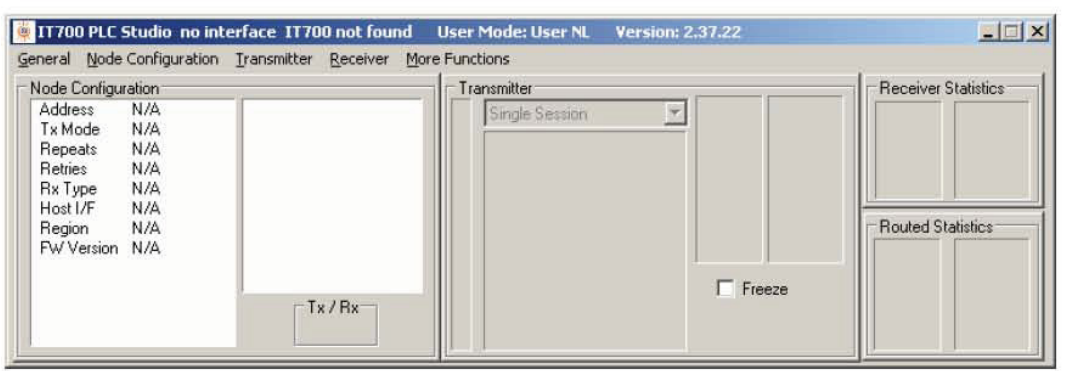

Step 5: Under the Title Transmitter select the option Connect. The following screen shot displays.

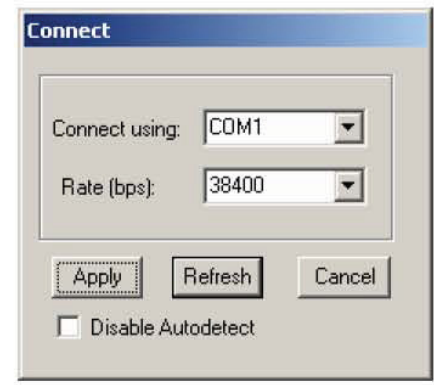

Step 6: Change the COM1 setting to COM11 keep the baud rate at 38400 . The following screen shot should display with all appropriate fields enabled. 
EMC Test Setup \& Operation for PLC Communication

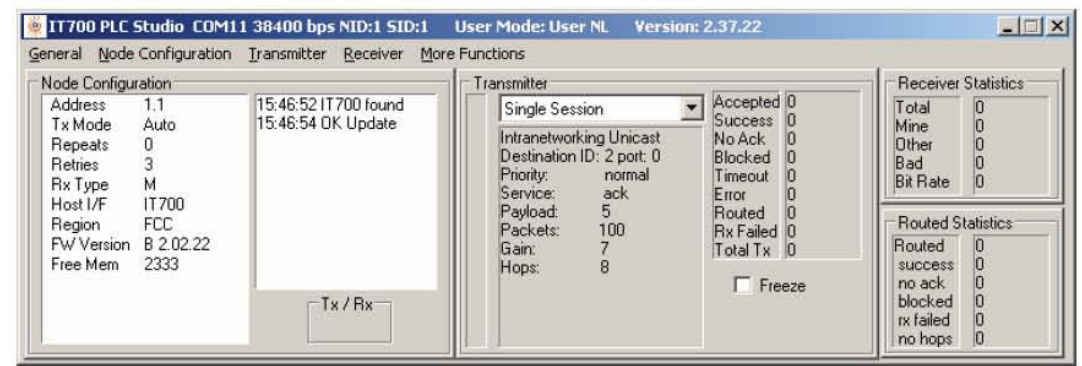

Phase I: Vitron AC Setup and Test Continued:

Step 7: Select The Transmitter option again the following screen shot displays.

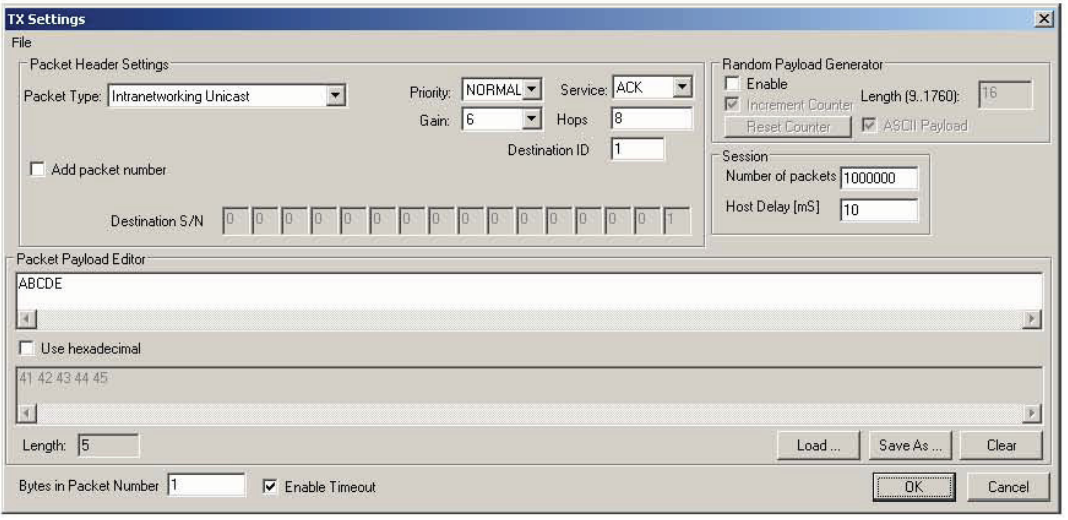

Step 8: Under the field Number of packets enter 100000 and under Host Delay enter 10. Fill in ABCDE under the Packet payload Editor. Click OK and the following screen shot should display. Observe the Light flashing RED under the Tx/Rx box.

\begin{tabular}{|c|c|c|c|c|c|c|}
\hline \multicolumn{3}{|c|}{ II 700 PLC Studio COM11 38400 bps NID;1 SID:1 } & User Mode: User NL. & Version: $\mathbf{2 . 3 7 . 2 2}$ & \multicolumn{2}{|r|}{$-|\square| x \mid$} \\
\hline \multicolumn{3}{|c|}{ General Node Configuration Iransmitter Receiver } & re Functions & & & \\
\hline \multicolumn{3}{|c|}{ Node Configuration- } & \multicolumn{2}{|l|}{ Transmitter } & \multicolumn{2}{|c|}{ Receiver Statistics } \\
\hline \multirow{8}{*}{$\begin{array}{l}\text { Address } \\
\text { Tx Mode } \\
\text { Repeats } \\
\text { Retries } \\
\text { Rx Type } \\
\text { Host I/F } \\
\text { Region } \\
\text { Fw Version } \\
\text { Free Mem }\end{array}$} & \multirow{8}{*}{$\begin{array}{l}1.1 \\
\text { Auto } \\
0 \\
3 \\
\text { M } \\
\text { IT700 } \\
\text { FCC } \\
\text { B 2.02.22 } \\
2333\end{array}$} & \multirow{7}{*}{$\begin{array}{l}\text { 15:46:52 I T700 found } \\
15.46 .54 \text { OK Update } \\
\text { 15:51:53 Send Packets } \\
\text { 15:52:37 Break } \\
15: 52.37 \text { Tr Session End } \\
\text { 15:52:54 Send Packets }\end{array}$} & \multirow{8}{*}{ 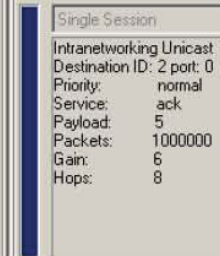 } & \multirow{6}{*}{$\begin{array}{ll}\text { Accepted } & 2452 \\
\text { Success } & 2266 \\
\text { No Ack } & 0 \\
\text { Blocked } & 167 \\
\text { Timeout } & 6 \\
\text { Entor } & 0 \\
\text { Routed } & 2433 \\
\text { Rx Failed } & 0 \\
\text { Total Tx } & 2305 \\
\end{array}$} & \multirow{4}{*}{$\begin{array}{l}\text { Total } \\
\text { Mine } \\
\text { Other } \\
\text { Bad } \\
\text { Bit Rate }\end{array}$} & \multirow{4}{*}{$\begin{array}{l}580 \\
580 \\
0 \\
0 \\
0.52 \\
\end{array}$} \\
\hline & & & & & & \\
\hline & & & & & & \\
\hline & & & & & & \\
\hline & & & & & \multicolumn{2}{|c|}{ Routed Statistics } \\
\hline & & & & & Routed & 0 \\
\hline & & & & & $\begin{array}{l}\text { success } \\
\text { no ack }\end{array}$ & $0_{0}^{0}$ \\
\hline & & $\mathrm{T}_{\mathrm{E}} / \mathrm{Rx}$ & & & $\begin{array}{l}\text { blocked } \\
\text { rx faled } \\
\text { no hops }\end{array}$ & $\begin{array}{l}0 \\
0 \\
0\end{array}$ \\
\hline
\end{tabular}


EMC Test Setup \& Operation for PLC Communication

Released $7-28$ - 2009

Document Rev 1.0

Phase I: Vitron AC Setup and Test Continued:

Step 9 Observation: Observe activity occurs on both Vitron Unit $1 \& 2$. See photos below for an illustration of transmit and receive activity.

Vitron \#1

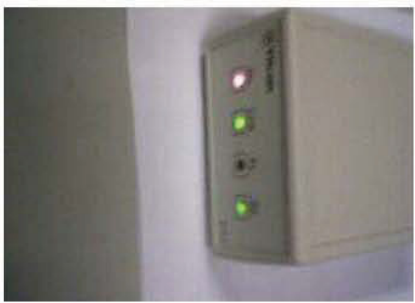

Vitron \#2

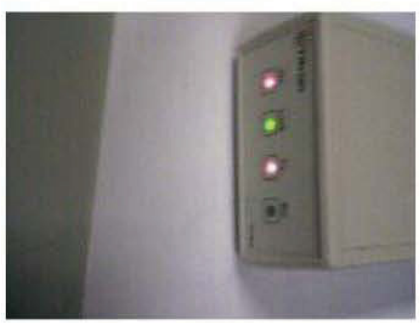

Summary: From this point on the devices are operating, EMC test measurements can now take place as called for on the test specification in the PO. When measurements are done, tear down the test setup by clicking exit under the General menu heading. Turn off the laptop, Vitron devices and then remove and place in a box ready for shipment back to eTec.

This Concludes Phase I 
EMC Test Setup \& Operation for PLC Communication

Released $7-28$ - 2009

Document Rev 1.0

Phase II: Vitron DC Setup and Test Continued:

Required Material: The following materials will be needed for setting up Phase II

- Two Vitron Units labeled 1,2

- One USB Cable

- Two DC Power Supplies for the Vitron Units.

- One Laptop Computer with Vitron Software

Procedure: Apply the following steps in setting up and operating the Vitron units in DC mode using the following steps and accompanying diagrams and drawings.

\section{Hardware Setup}

Step 1: Using Figure 2.0 located on page 1 of 2 on the drawing titled, "EMC Test Layout" as a reference connect Vitron 1 to the test laptop using a USB cable.

Step 2: Connect Vitron 1 to its power adapter and plug the power adapter into a DC Power Supply check the LED light illuminates before preceeding further, if it fails to light up, the battery needs to be recharged and testing will need to take place the following day.

Step 3: Connect Vitron 2 to its power adapter and plug it into another DC power supply. Again if the LED fails to illuminate, the DC power supply needs to recharge.

Observes Vitron 2 does not have a USB cable connected to the laptop. This is a deliberate action given the interest in measuring PLC communication exclusively.

This completes setting up the hardware for this Phase.

Test Operation Section: Continue with steps $4-9$ as described in Phase I.

\section{This Concludes Phase II}


EMC Test Setup \& Operation for PLC Communication

Phase III: Ethernet Intellon Device

Required Material: The following materials will be needed for setting up Phase III

- Two Intellon AV Ethernet devices

- Two Ethernet Cables

- Two Laptops.

Procedure: Apply the following steps in setting up and operating the Homeplug AV Ethernet devices.

\section{Hardware Setup}

Step 1: Using Figure 3.0 located on page 2 of 2 on the drawing titled, "EMC_Test Layout". Plug in each device into two separate receptacles.

Step 2: Connect an Ethernet cable from laptop 1 to Intellon Ethernet device \#1.

Step 3: Repeat Step 2 for Laptop 2 and Intellon AV Ethernet device \#2.

This Completes setting up the hardware for this phase.

\section{Software Setup}

Step 4: Turn on test laptop $1 \& 2$ and assign the following

$$
\begin{array}{r}
\text { Laptop \#1 IP Address }(\text { static })=192.168 .0 .100 \\
\text { Default Gateway }=192.168 .0 .100 \\
\text { Subnet Mask }=255.255 .255 .0 \\
\text { Laptop \#2 IP Address(static) }=192.168 .0 .101 \\
\text { Default Gateway }=192.168 .0 .101 \\
\text { Subnet Mask }=255.255 .255 .0 \\
\{\text { Keep the workgroup. }\}
\end{array}
$$

Step 5: Transfer a large file from Laptop 1 to Laptop 2. Conduct EMC measurements during the file transfer. Use the CAD Video File.

\section{This Concludes Phase III}


EMC Test Setup \& Operation for PLC Communication

Phase IV: Maxim 2990 Test setup \& Operation:

Required Material: The following materials will be needed for setting up Phase IV

- Two Maxim 2990 devices

- Two Serial Cables

- Two Laptops.

- Power Accessories Kit

- Serial to Fiber converters

Procedure: Apply the following steps in setting up and operating the Maxim 2990 devices for 110 Vac test operation.

\section{Hardware Setup}

Step 1: Examine Figure 6.0 located on page 1 of 2 on the drawing titled, "EMC Maxim 2990". Using modules contained the power accessories kit, create two $110 \mathrm{Vac}$ plugs.

Step 2: Connect the cold wires to DUT\#1 and DUT $\# 2$ on as illustrated in Figure 6.0. Use the overall layout snapshot located below figure 6.0 as a guide.

Step 3: Connect the cold wires by matching the correct polarity with jumper wires colored as red $=+$ and Black $=-$.

Step 4: Examine the LED flash rate between DUT\#1 and Dut $\# 2$. There should be two flashing lights operating in an alternating sequence. This indicates there is packet data being transferred between DUT\#1 and DUT\#2 .

Step 5: Connect both laptops\#1\&2 to DUT\#1\&2 respectively, using a serial cable as See figure 6.0 on the "EMC Maxim 2990" drawing. 
EMC Test Setup \& Operation for PLC Communication

Released 7-28 - 2009

Document Rev 1.0

\section{Software Setup}

Step 6: Turn on laptop 1 \& 2 and select the program icon " $t 134$ mpro" on both laptops see illustration below:

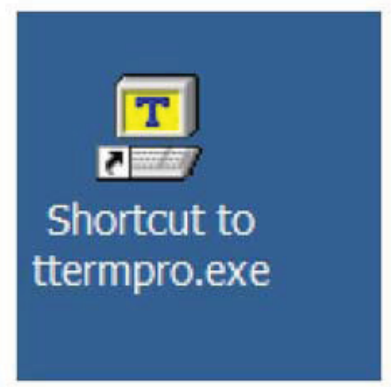

Step 7: After selecting the icon, the following dialogue box appears illustrating packet data transfer between DUT \#1 and DUT\#2

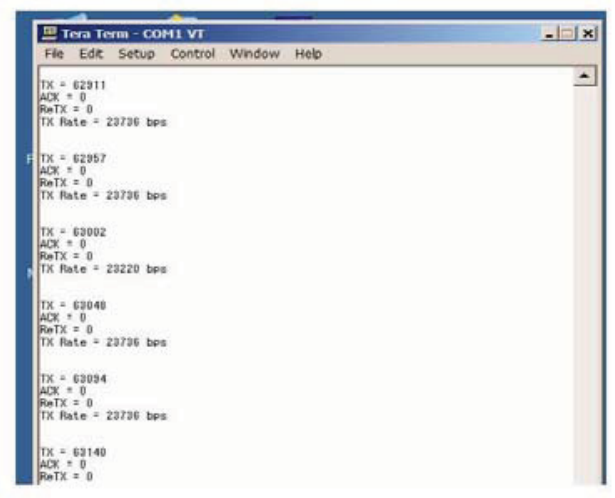

Step 8: Verify traffic data is real by disconnecting one of the cold wires and observing all traffic activity STOPS between both laptops. 
EMC Test Setup \& Operation for PLC Communication

\section{This Concludes Phase IV}

Phase V: Maxim 2990 Test setup \& Operation 12V dc:

Required Material: The following materials will be needed for setting up Phase V

- Two Maxim 2990 devices

- Two Serial Cables

- Two Laptops.

- Power Accessories Kit

- Serial to Fiber converters

- $12 \mathrm{~V}$ de Power Supply

Procedure: Apply the following steps in setting up and operating the Maxim 2990 devices for 12 Vdc test operation.

\section{Hardware Setup}

Step 1: Examine Figure 6.0 located on page 1 of 2 on the drawing titled, "EMC Maxim 2990". Using modules contained the power accessories kit, select the 100 to $200 \mathrm{Vac}$ input to $12 \mathrm{Vdc}$ output power adapter, create the plug for either $110 \mathrm{Vac}$ or a $220 \mathrm{Vac}$ receptacle.

Step 2: Connect the other end into the $12 \mathrm{Vdc}$ receptacle on each DUT.

Step 3: Connect the cold wires to DUT\#1 and DUT\#2 on as illustrated in Figure 6.0. Use the overall layout snapshot located below figure 6.0 as a guide.

Step 4: Connect the cold wires by matching the correct polarity with jumper wires colored as red $=+$ and Black $=-$.

Step 5: Examine the LED flash rate between DUT\#1 and Dut $\# 2$. There should be two flashing lights operating in an alternating sequence. This indicates there is packet data being transferred between DUT\#1 and DUT\#2.

Step 6: Connect both laptops\#1\&2 to DUT\#1\&2 respectively, using a serial cable as See figure 6.0 on the "EMC Maxim 2990" drawing. 
EMC Test Setup \& Operation for PLC Communication

Released $7-28$ - 2009

Document Rev 1.0

\section{Software Setup}

Step 6: Turn on laptop $1 \& 2$ and select the program icon "tt34mpro" on both laptops see illustration below:

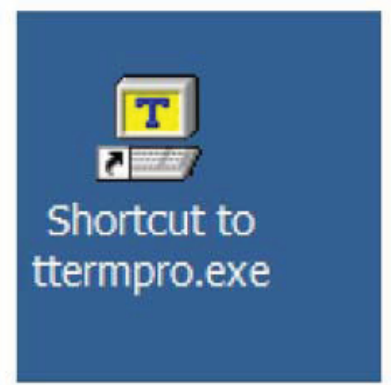

Step 7: After selecting the icon, the following dialogue box appears illustrating packet data transfer between DUT \#1 and DUT\#2

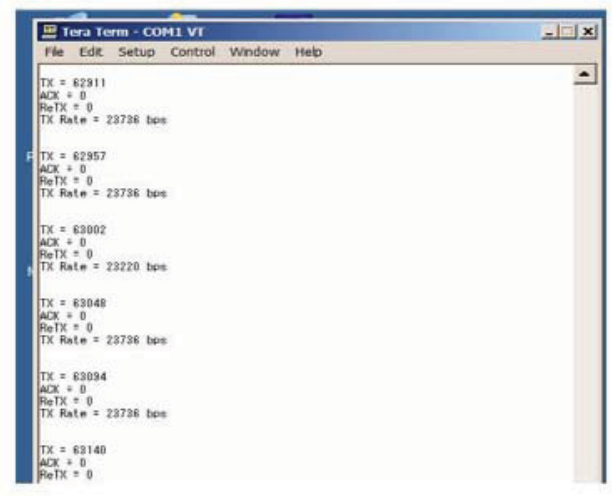

Step 8: Verify traffic data is real by disconnecting one of the cold wires and observing all traffic activity STOPS between both laptops.

\section{This Concludes Phase V}


EMC Test Setup \& Operation for PLC Communication

Released $7-28-2009$

Document Rev 1.0

This Concludes Test Setup and Operation Procedure for PLC Communications. 


\section{Appendix A-9 Durability Test Setup Drawings (Maxim 2990/2991)}

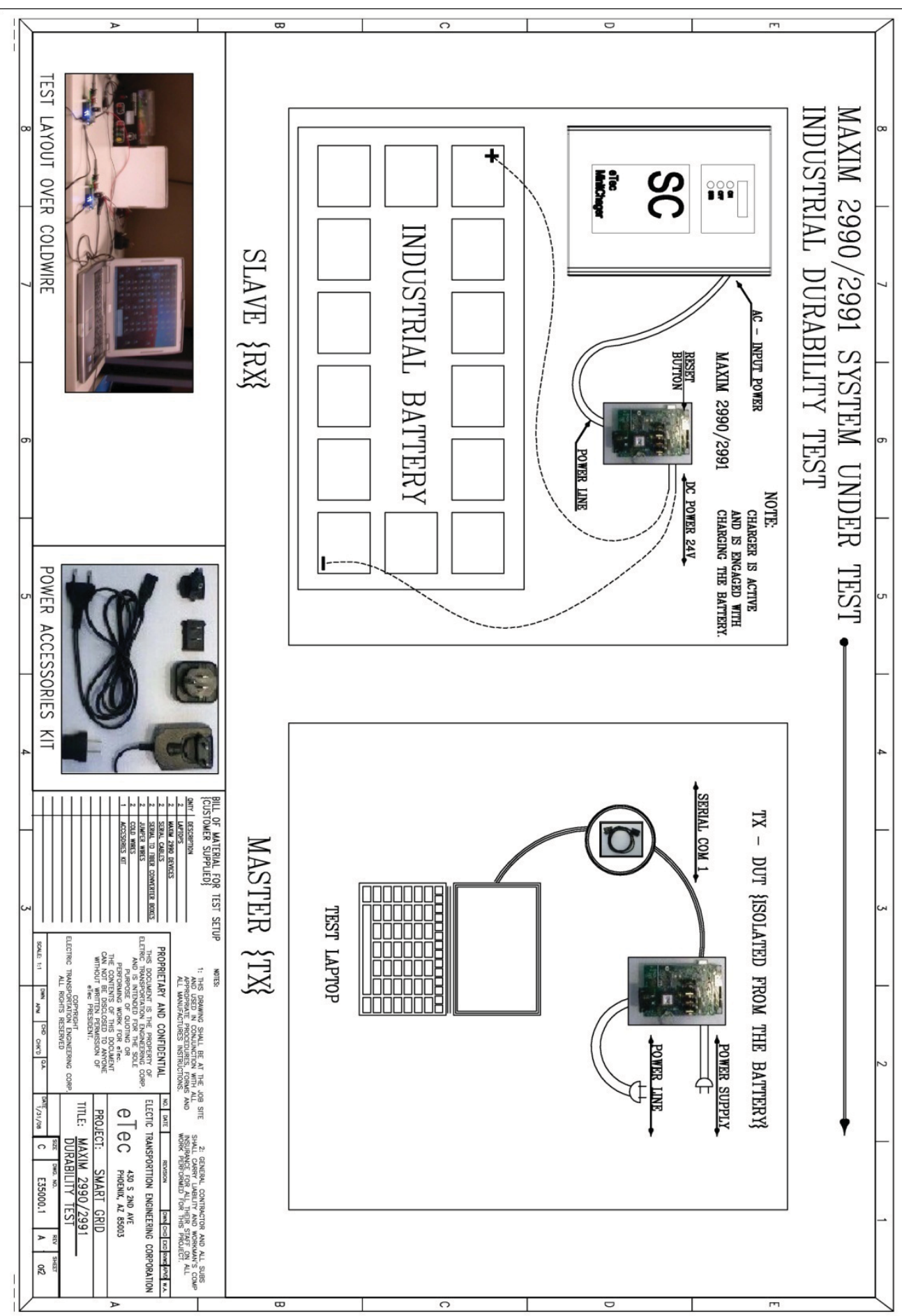




\title{
Appendix A-10 Durability Test Setup Instructions (Maxim 2990/2991)
}

\author{
***Durability Functional Test Instructions for Maxim 2990/2991 EV kit*** \\ Released 8-1 - 2009 \\ Document Rev 1.0
}

Preliminary: The purpose of this test is to measure Maxim 2990/2991 perform under typical off road charging conditions. The setup shall comprise of Minit Charger Fast Charging Systems and associated battery hardware. Equipment list used for testing is as follows:

- eTec Minit Fast SC Charger

- 24V DC Hawker battery

- Associated charging cables

- Battery Device Controller BDC

- Maxim 2990/2991 EV kit

- Two test laptops

Physical Setup: Consists of two hardware setups once for the EV kit validation and the other for Charger setup validation. Verify safety by examining the area for potential safety hazards, such as broken wires, damaged equipment, excess debris, battery condition, and other related items. If all safety items are met proceed to the next series steps observing each in great detail.

\section{Maxim 2990/2991 EV Kit (Slave)}

- Charger Setup Validation: With the power off, remove the SC charger case and locate the $\mathrm{AC}$ input lines.

- Using a prefabricated $\mathrm{AC}$ power line with ring terminals, connect each ring to the correct node on the input terminal.

- Since the SC charger was used on a $24 \mathrm{~V}$ battery, an adaptor cable was required between the battery cable and the charger cables. Mate the cables by match their respective $\mathrm{EBC}$ connectors.

- Connect the Maxim EV modified power line with ring terminal to the respective $+/$ - terminals. (This is the DC power used in supporting rail to rail voltage not the AC power line.)

- Connect $\mathrm{AC}$ Power from the charger $\mathrm{AC}$ input line to the slave (RX) of the Maxim 2990/2991 EV kit. 
***Durability Functional Test Instructions for Maxim 2990/2991 EV kit***

Released 8-1 - 2009

Document Rev 1.0

Physical Setup Continued

\section{Maxim 2990/2991 EV Kit (Master)}

- Connect the Maxim 2990/2991 EV kit (Master) by first selecting an AC power line that draws the same power as the eTec Minit Fast charger.

- Connect rail to rail power using a $9 \mathrm{~V}-12 \mathrm{~V}$ battery source, in like wise fashion to the Maxim 2990/2991 EV kit (Slave).

- Connect a Test laptop to the Maxim 2990/2991 EV (Master) kit using a serial cable connection.

\section{Observation of Physical Connectivity}

- Observe both modules illuminate with blue LED's flashing in an alternating sequence.

- After five minutes observe an alternating illumination of green LED's with no RED color in the sequence.

- On the Test laptop, open the Termpro console application and observe the data traffic.

- Connect an Oscilloscope to the Transmit pin on the Slave device and observe signal packet data. Adjust the frequency and time division accordingly until a clear visual is displayed on the screen.

\section{Operational Execution of Durability Test.}

- With the physical test validated and the charger ready turn on the charger by pressing the start button. The screen on the charger should illuminate with no errors, or messages saying "need service".

- Using a current probe, monitory the current draw on the charger display matches what is being measured by the current probe. The current probe should be measuring the DC charge cable connected to the battery.

- Observe the behavior on the Test laptop paying close attention to the error rate. Also, examine the LED behavior of the status LED's on the master and slave devices. Finally, as a backup observe the signal behavior on the oscilloscope for noise, especially as the charger begins to execute switching power in ramping up current to the battery.

- Be ready to terminate the test if any safety issues should arise. Collect the data in reparation of a report. 
Appendix B

\section{Data Results}




\section{Appendix B-1 \\ Functional Test Results (Yitran IT700)}

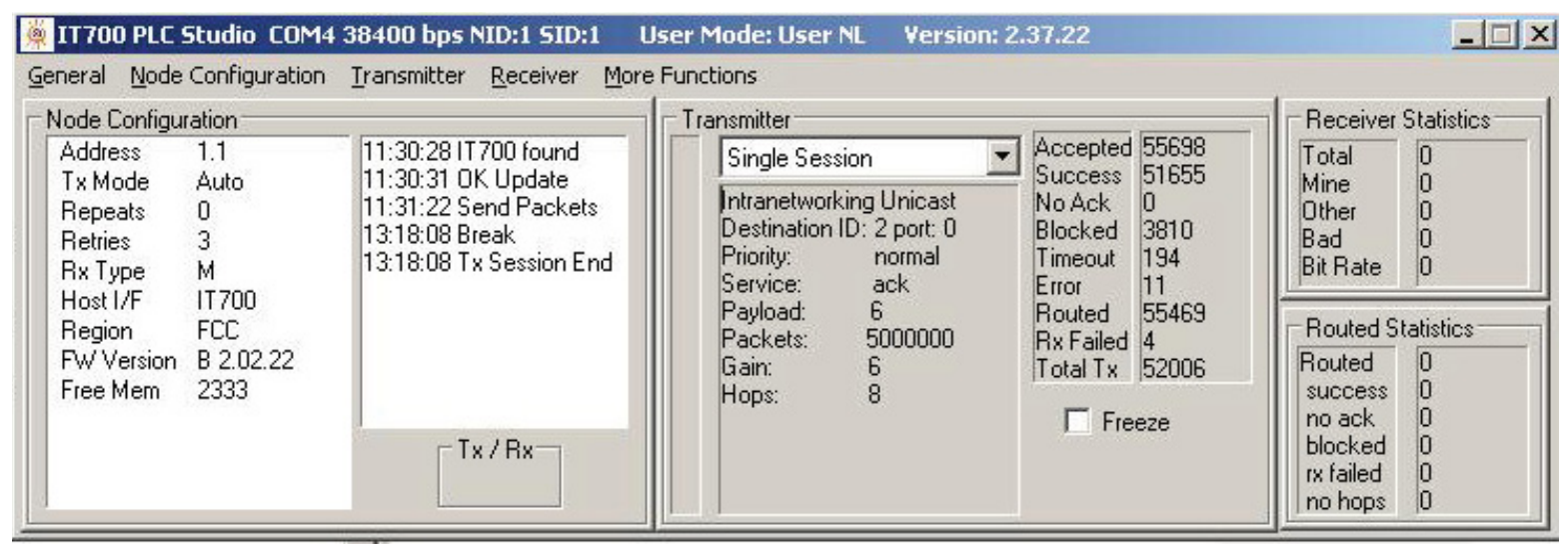

Yitran IT700 Functional Test Data Report connected to PHEV Hymotion Prius. 


\section{Appendix B-2 \\ Signal Analysis Report (Yitran IT700)}

\section{Test Setup}

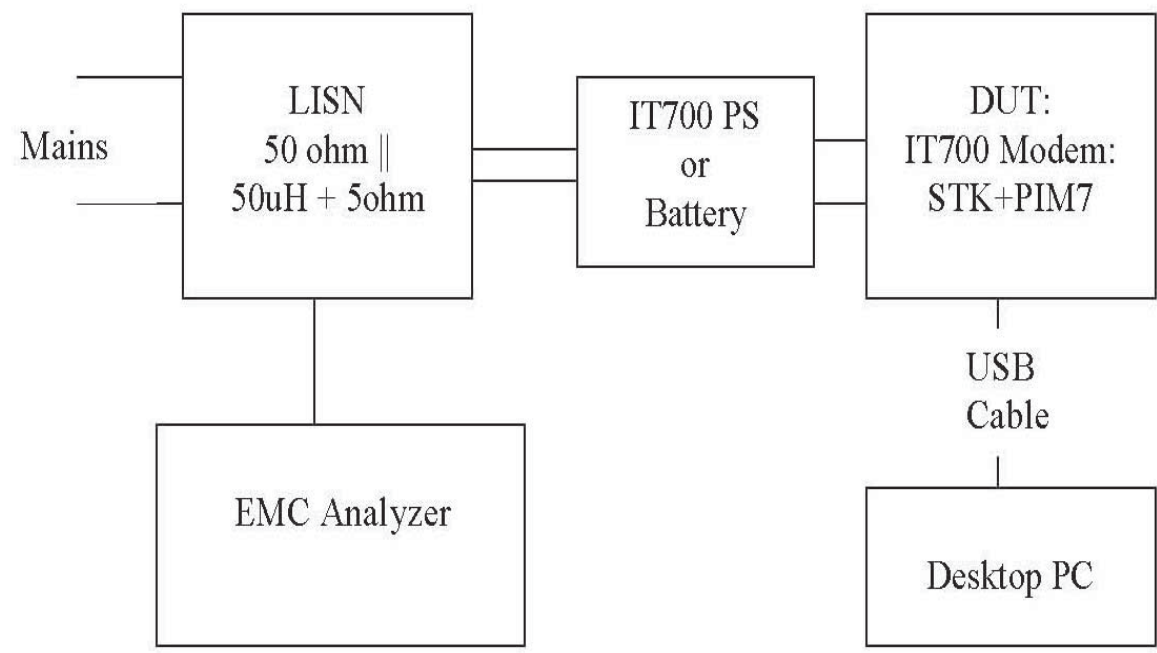

Notes:

- Modem was tested with both Yitran standard universal power supply and a special set that included a battery for powering modem and IT700 power line coupler for PLC signal transmission.

- Modem transmission mode (set with Host application):

- ARA disabled

- Internetworking Broadcast

- Power Level $=6$

- Transmission duty cycle was set to max possible value:

- Payload $=100$

- Host Delay $=0$

- $\mathrm{TTL}=15000 \mathrm{~ms}$

- All equipment was properly earthed through wall sockets

- Two units (STK + Modem) were tested 


\section{TX Spectrum Measurements}

\section{Typical TX Spectrums with Switching Mode Power Supply}

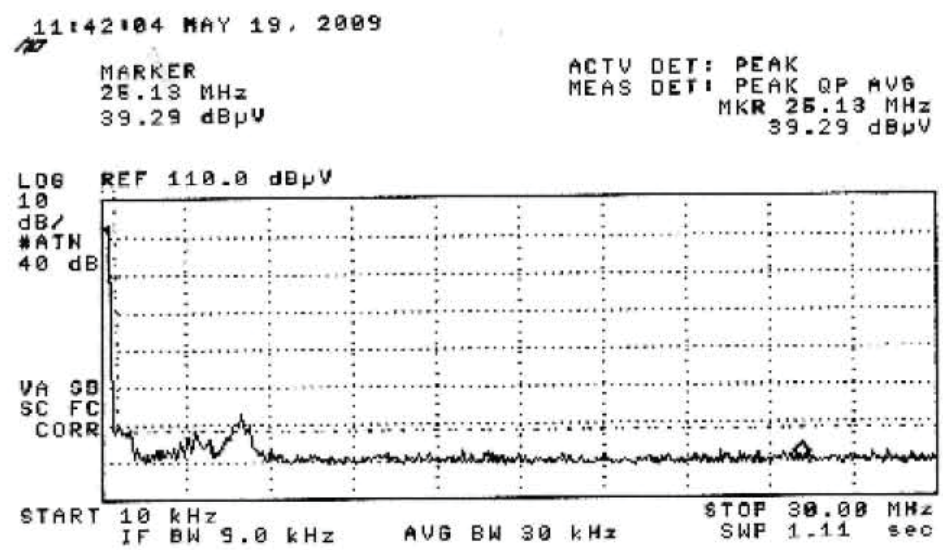

The picture above shows typical spectrum recorded through SMPS unit with frequency sweep from $10 \mathrm{kHz}$ to $30 \mathrm{MHz}$. Limit $(250 \mathrm{uV}=48 \mathrm{dBuV})$ is set by the dashed line. One can easily see a noise "hump" at $5 \mathrm{MHz}$ neighborhood that violates $48 \mathrm{dBuV}$ limit. This "violation" is caused by USB connection as we saw earlier. Therefore it was excluded from further consideration.

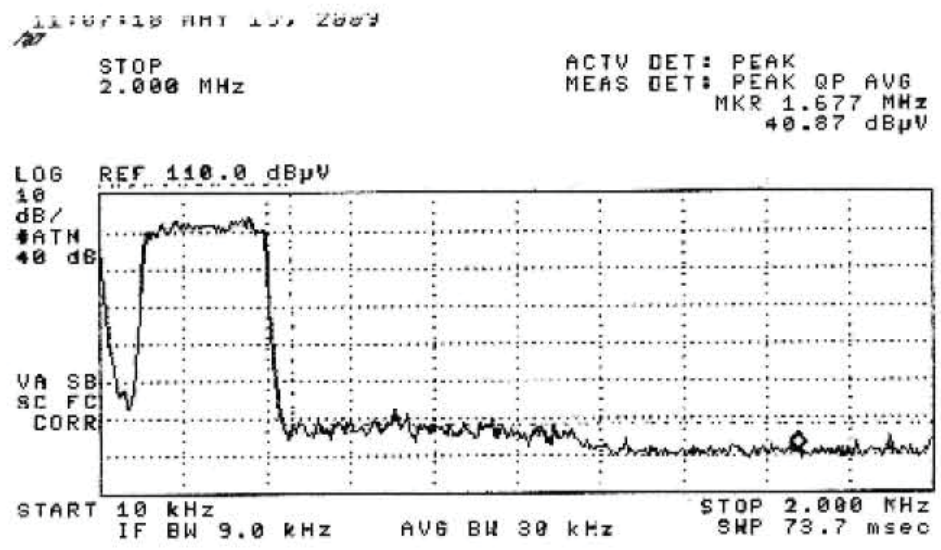

The picture above shows typical spectrum recorded through SMPS with frequency sweep from $10 \mathrm{kHz}$ to $2 \mathrm{MHz}$. Limit $(250 \mathrm{uV}=48 \mathrm{dBuV})$ is set by the dashed line. One can see that some parts of the spectrum at frequencies between $500 \mathrm{kHz}$ and $1 \mathrm{MHz}$ cross the limit line. Those frequencies are considered as "suspicious" and measured precisely with quasi peak and average detectors. The measurements results are shown in the picture below: 

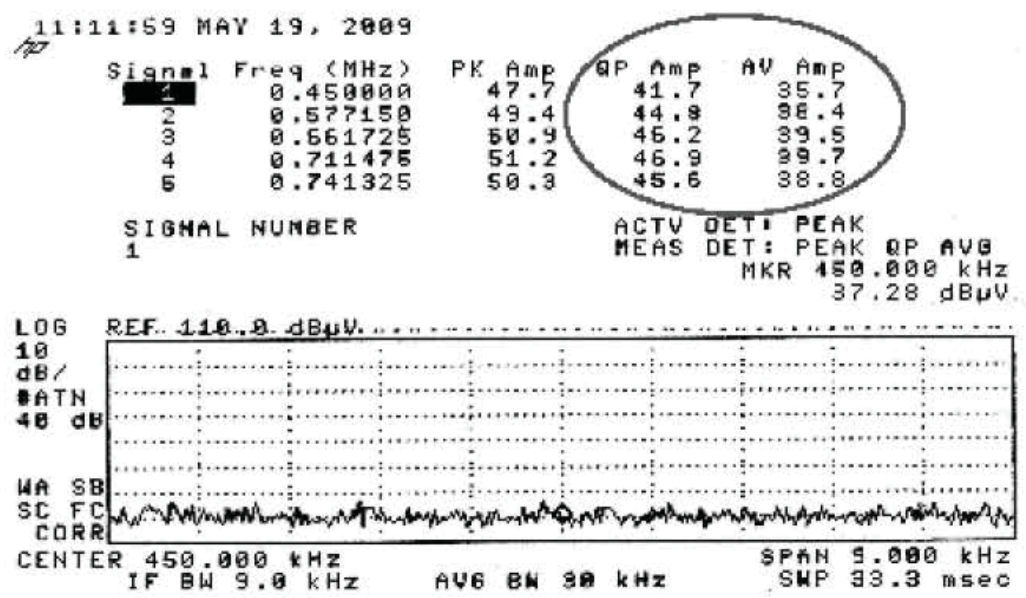

Quasi peak measurements do not show $48 \mathrm{dBuV}$ limit violation.

\section{Typical TX Spectrums with Battery Unit}

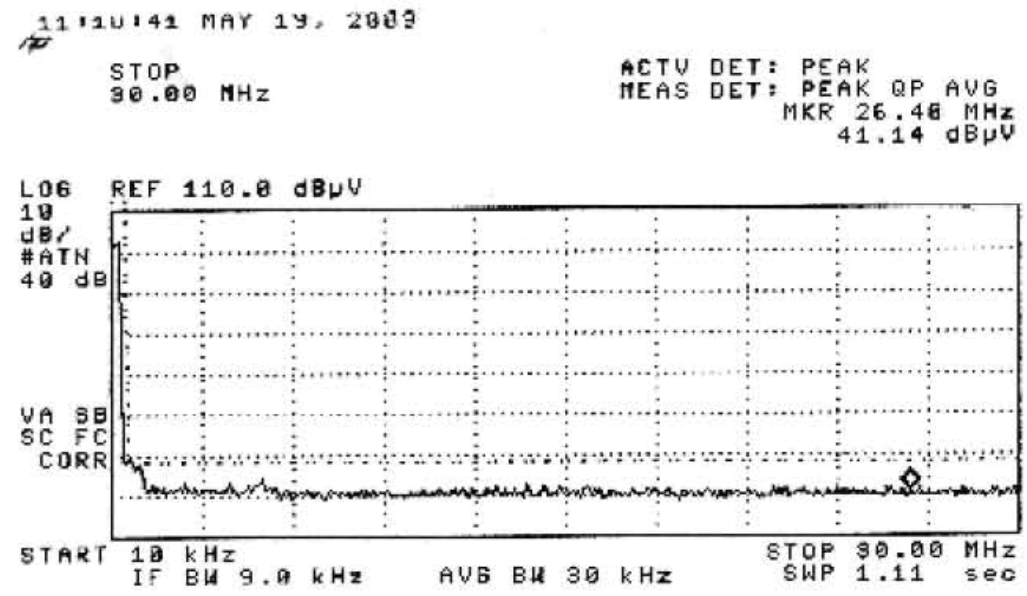

The picture above shows typical spectrum recorded through battery unit with freq sweep from $10 \mathrm{kHz}$ to $30 \mathrm{MHz}$. Limit $(250 \mathrm{uV}=48 \mathrm{dBuV})$ is shown by the dashed One can see that no part of spectrum crosses limit at frequencies above 1-2 MHz. Moreover this part of spectrum has good spare of $5-8 \mathrm{dBuV}$. 


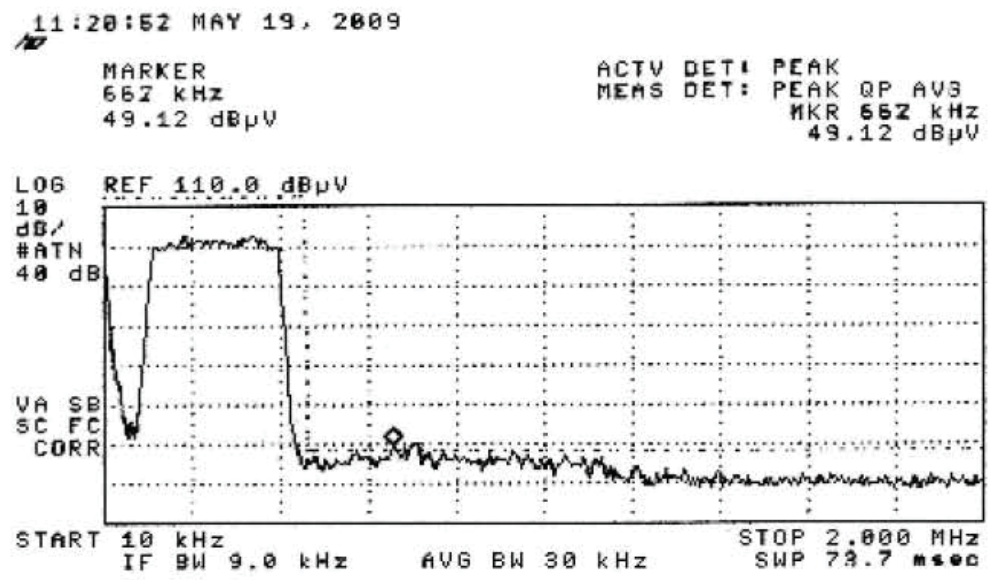

The picture above shows typical spectrum recorded through battery unit with frequency sweep from $10 \mathrm{kHz}$ to $2 \mathrm{MHz}$. Limit $(250 \mathrm{uV}=48 \mathrm{dBuV})$ is shown by the dashed line. One can see in general TX spectrum looks "cleaner" than in previous case with SMPS but some parts of the spectrum still cross the limit line. Those frequencies are considered as "suspicious" and measured precisely with quasi peak and average detectors. The measurements results are shown in the picture below:

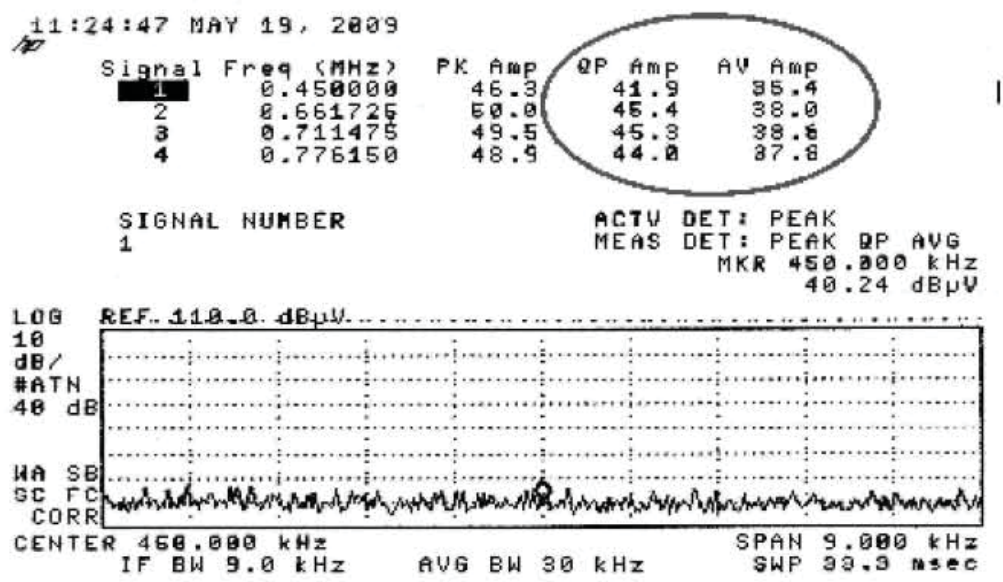

Quasi peak measurements do not violate limit of $48 \mathrm{dBuV}$ and have better spares.

\section{Conclusion}

- IT700 modem is able to pass SAE551-5 requirements at TX Power Level $=6$ with spare of $2.5-3 \mathrm{~dB}$ in the worst case.

- Yitran SMPS when used together with USB connection to PC (or opposite, USB connection paired with Yitran SMPS) causes very specific noise in $5 \mathrm{MHz}$ neighborhood.

- Battery unit has much lower background noise and almost does not create specific "USB-related" one. 


\section{Appendix B-3 \\ Functional Test Results (Maxim 2990/2991)}
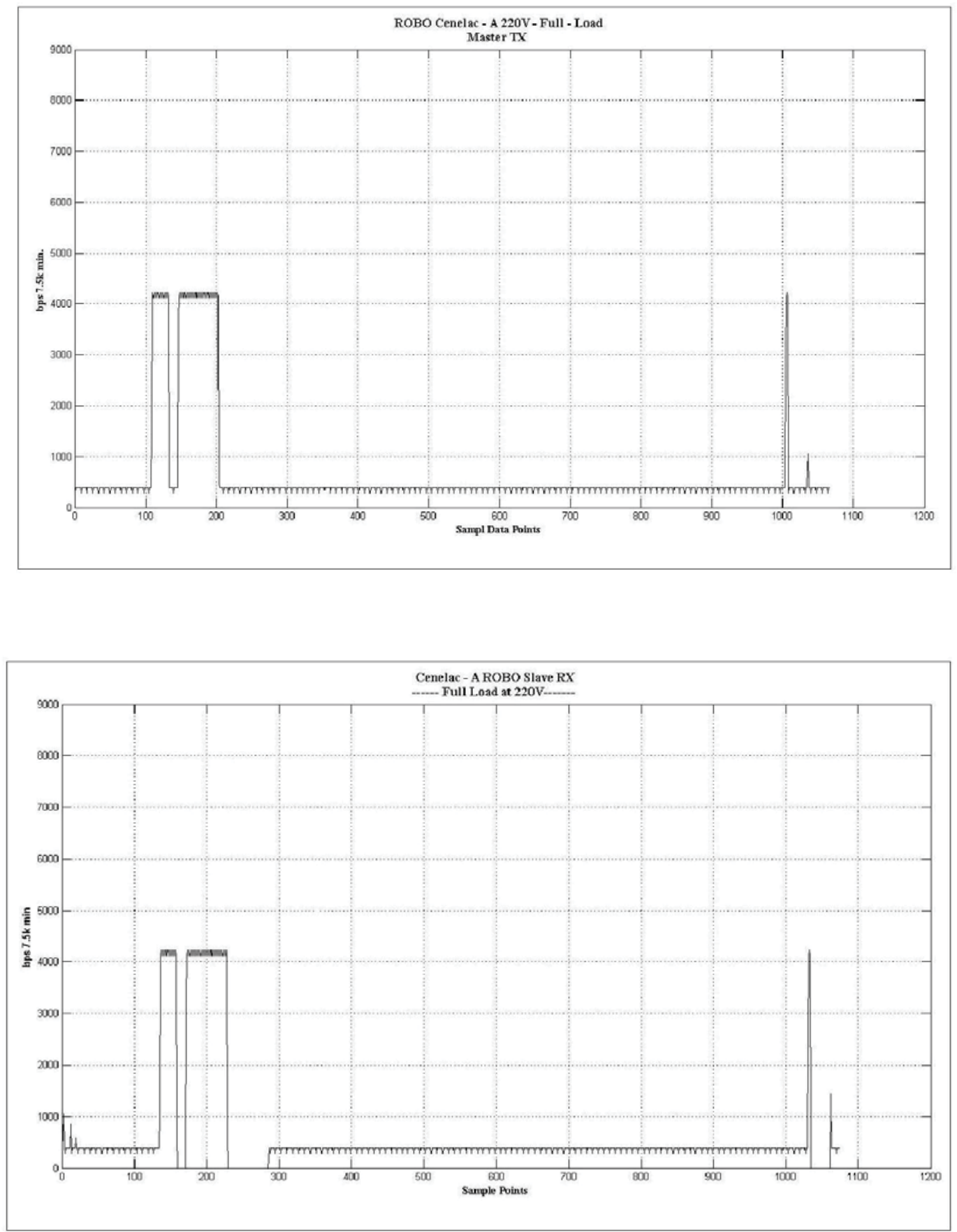


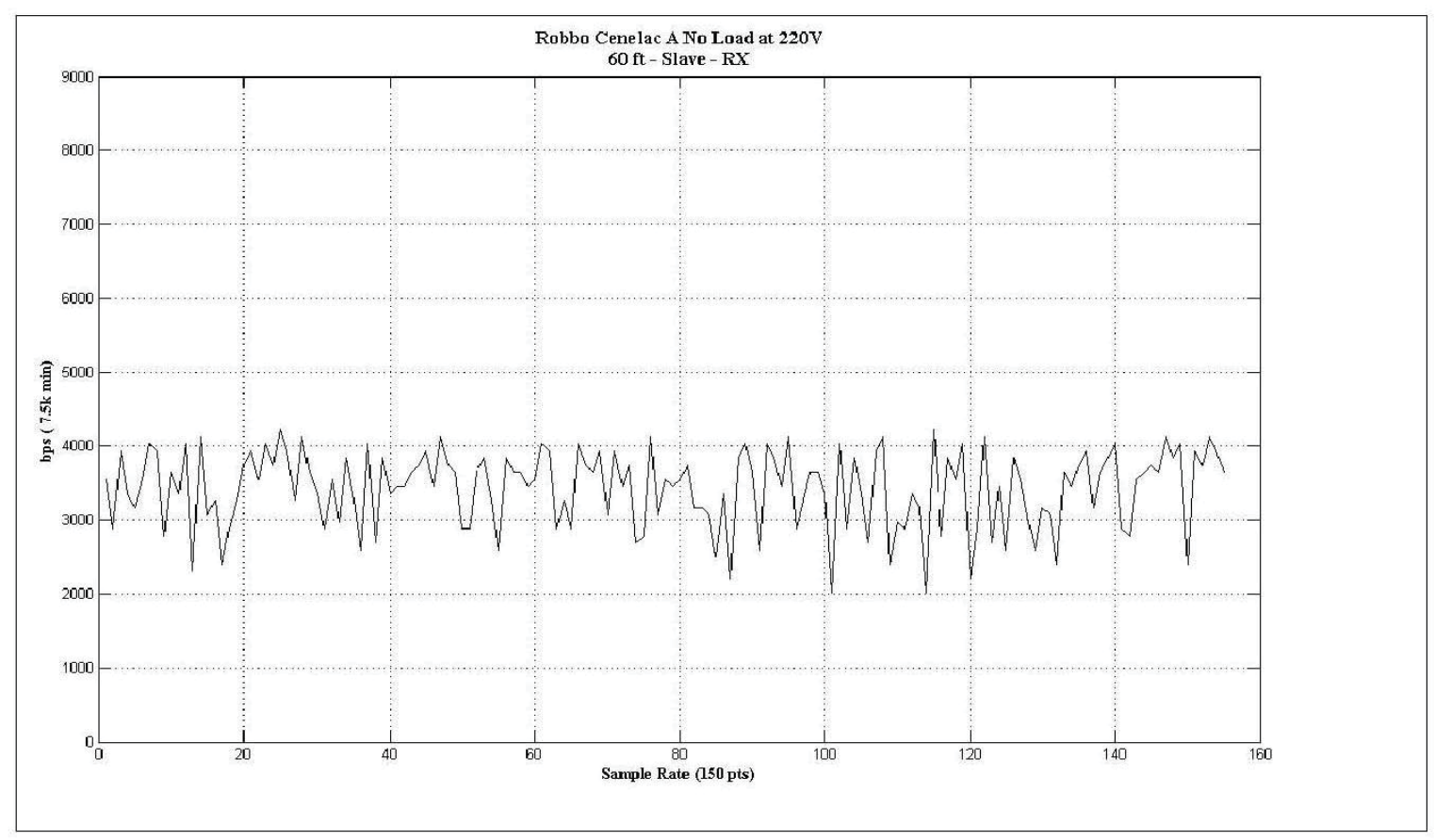




\section{Appendix B-4 \\ Functional Test Results (Intellon XAV101)}
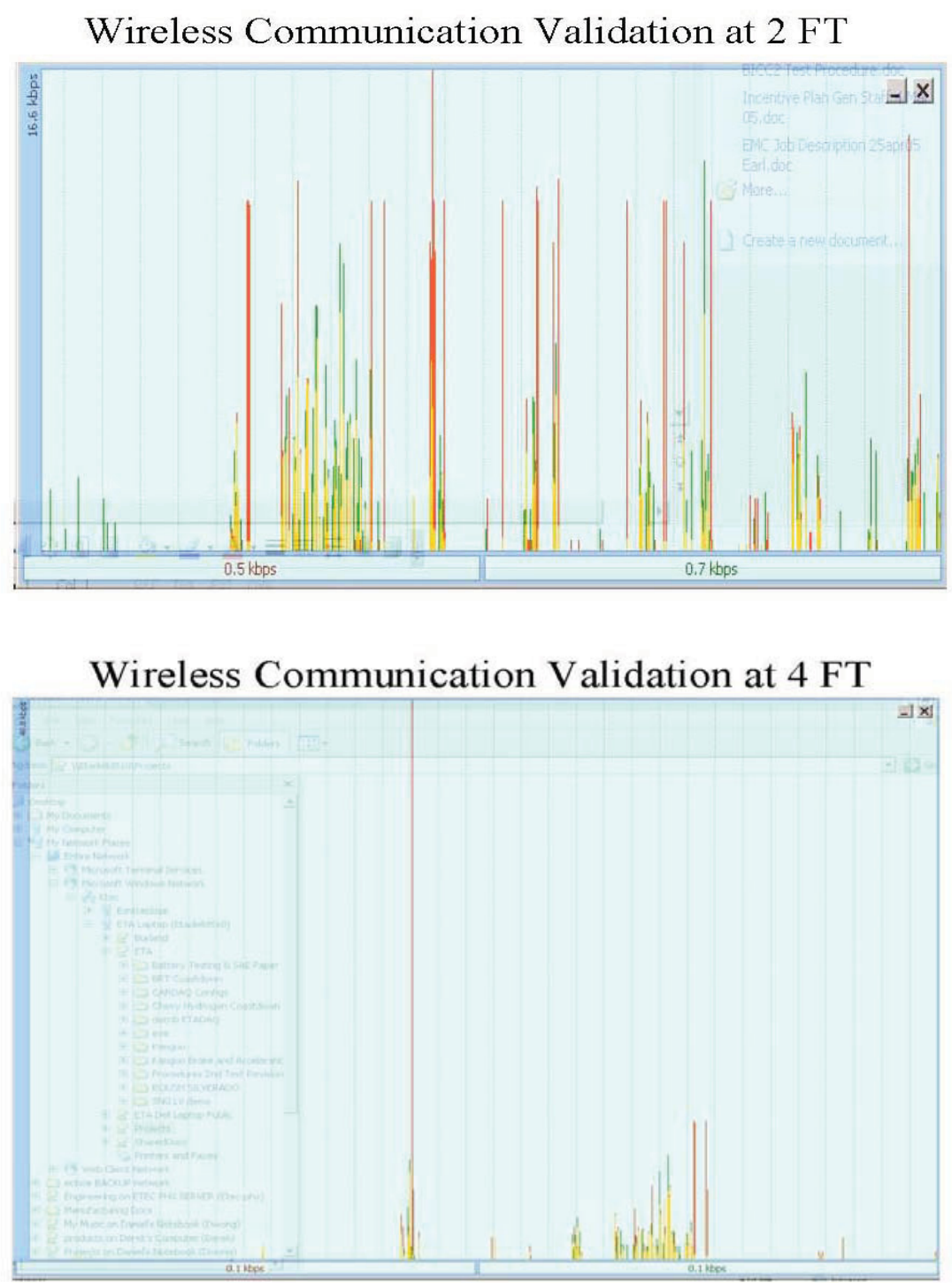
Wireless Communication Validation at 6 FT

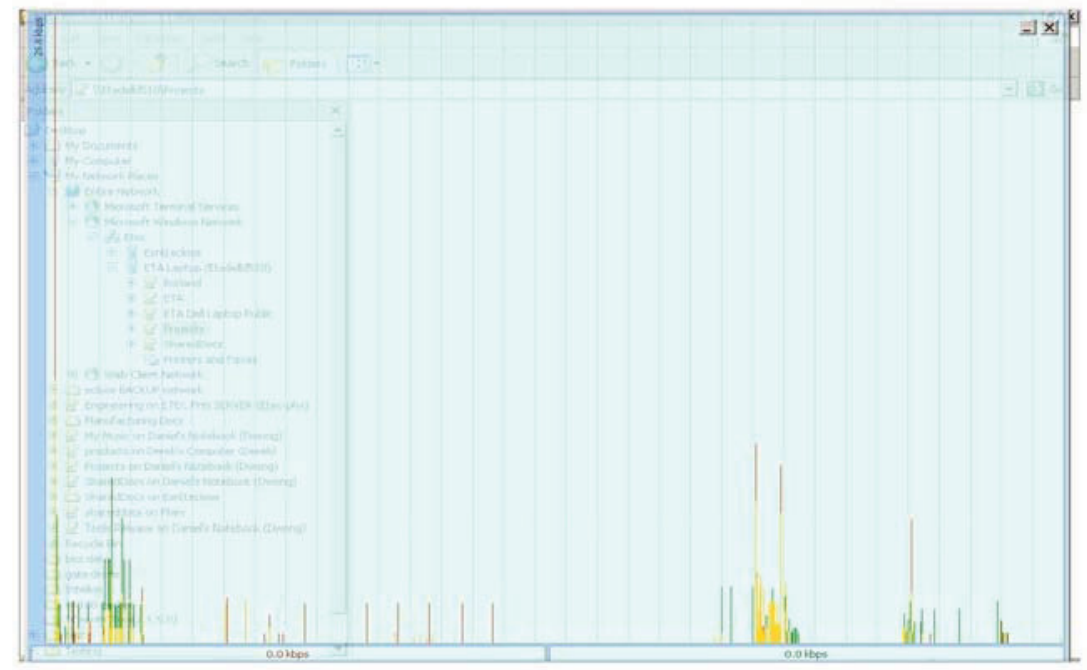




\title{
Appendix B-5 \\ Electromagnetic Capability Test Instructions at Electric Transportation Applications Engineering Center
}

\author{
EMC Test Setup \& Operation for PLC Communication \\ Released $7-28-2009$ \\ Document Rev 1.0
}

Preliminary: The EMC Test setup and operation consists of four test configurations, and a test operation for each one. Test configurations are broken down as follows:

- Vitron AC Setup \& Operation Phase I:

- Vitron DC Setup \& Operation Phase II:

- Interlon: Ethernet over Powerline Phase III:

- MAXIM PLC 2990 AC 110 \& 240 Hot Line:

- MAXIM PLC 2990 12V DC Setup \& Operation Phase V:

Instruction: Each phase consists of instructions and references to drawings, manuals and screen shots as they apply to the test operation and setup.

Phase I: Vitron AC Setup and Test:

Required Material: The following materials will be needed for setting up Phase I

- Two Vitron Units labeled 1,2

- One USB Cable

- Two AC Power Supplies for the Vitron Units.

- One Laptop Computer with Vitron Software

Procedure: Apply the following steps in setting up and operating the Vitron units in AC mode using the following steps and accompanying diagrams and drawings.

\section{Hardware Setup}

Step 1: Using Figure 1.0 located on page 1 of 2 on the drawing titled, "EMC_Test Layout" as a reference connect Vitron 1 to the test laptop using a USB cable.

Step 2: Connect Vitron 1 to its power adapter and plug the power adapter into a receptacle.

Step 3: Connect Vitron 2 to its power adapter and plug it into a separate receptacle away from where Vitron 1 is connected. Do not plug both Vitron 1 and Vitron 2 into the same receptacle. 
EMC Test Setup \& Operation for PLC Communication

Released $7-28$ - 2009

Document Rev 1.0

Observes Vitron 2 does not have a USB cable connected to the laptop. This is a deliberate action given the interest in measuring PLC communication exclusively.

This completes setting up the hardware for this Phase.

Phase I: Vitron AC Setup and Test Continued:

Step 4: Turn on the test laptop and turn on Vitron 1. Select Vitron Icon on the desktop. This starts the Test software specially made to communicate with Vitron. The following screen shot should display.

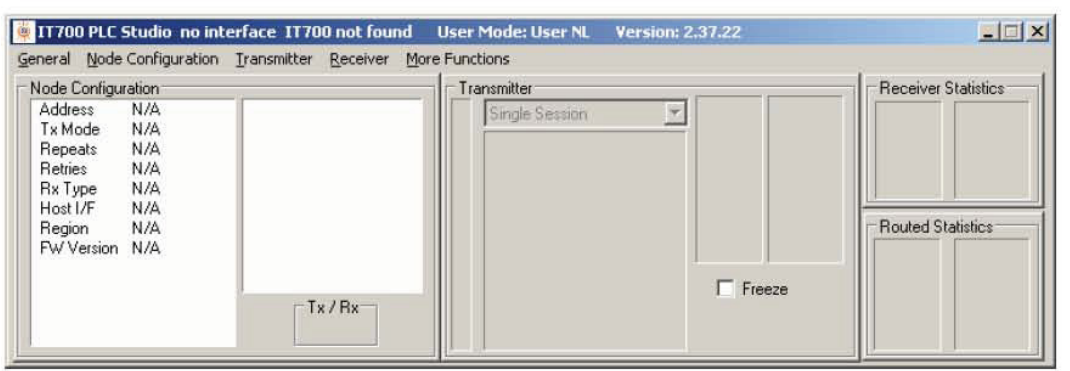

Step 5: Under the Title Transmitter select the option Connect. The following screen shot displays.

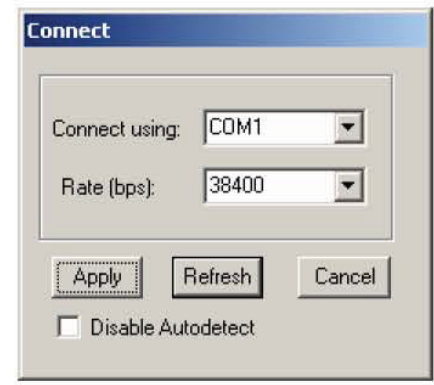

Step 6: Change the COM1 setting to COM11 keep the baud rate at 38400 . The following screen shot should display with all appropriate fields enabled. 
EMC Test Setup \& Operation for PLC Communication

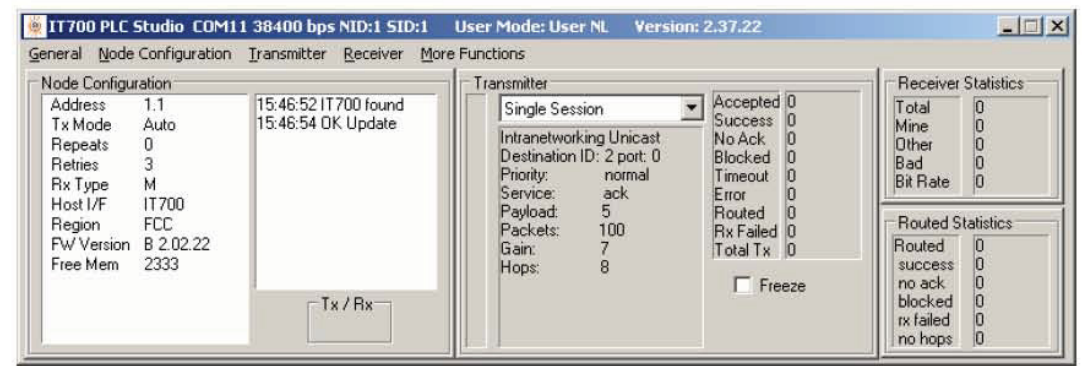

Phase I: Vitron AC Setup and Test Continued:

Step 7: Select The Transmitter option again the following screen shot displays.

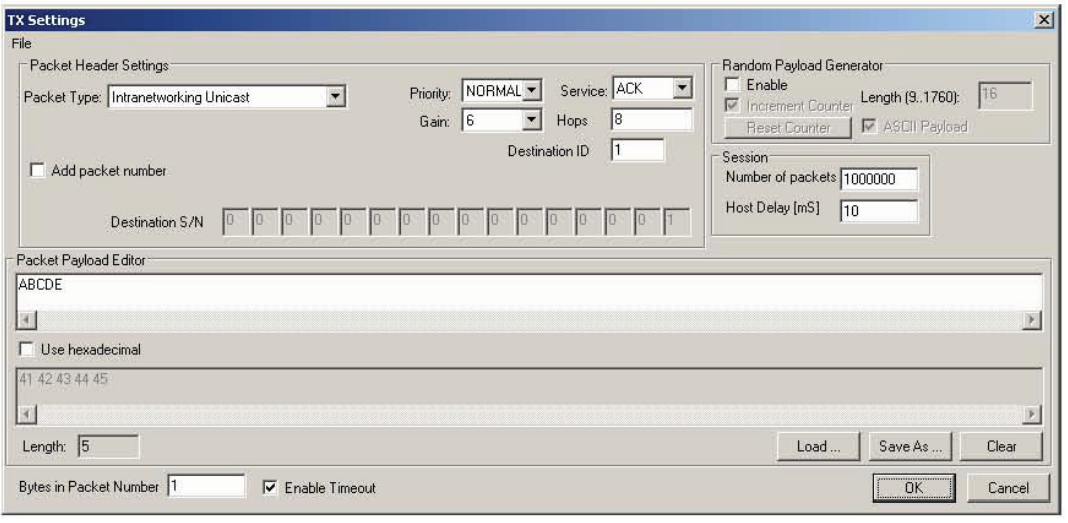

Step 8: Under the field Number of packets enter 100000 and under Host Delay enter 10. Fill in ABCDE under the Packet payload Editor. Click OK and the following screen shot should display. Observe the Light flashing RED under the Tx/Rx box.

\begin{tabular}{|c|c|c|c|c|c|c|}
\hline \multicolumn{3}{|c|}{ II 700 PLC Studio COM11 38400 bps NID;1 SID:1 } & User Mode: User NL. & Version: $\mathbf{2 . 3 7 . 2 2}$ & \multicolumn{2}{|r|}{$-|\square| x \mid$} \\
\hline \multicolumn{3}{|c|}{ General Node Configuration Iransmitter Receiver } & re Functions & & & \\
\hline \multicolumn{3}{|c|}{ Node Configuration- } & \multicolumn{2}{|l|}{ Transmitter } & \multicolumn{2}{|c|}{ Receiver Statistics } \\
\hline \multirow{8}{*}{$\begin{array}{l}\text { Address } \\
\text { Tx Mode } \\
\text { Repeats } \\
\text { Retries } \\
\text { Rx Type } \\
\text { Host I/F } \\
\text { Region } \\
\text { Fw Version } \\
\text { Free Mem }\end{array}$} & \multirow{8}{*}{$\begin{array}{l}1.1 \\
\text { Auto } \\
0 \\
3 \\
\text { M } \\
\text { IT700 } \\
\text { FCC } \\
\text { B 2.02.22 } \\
2333\end{array}$} & \multirow{7}{*}{$\begin{array}{l}\text { 15:46:52 I T700 found } \\
15.46 .54 \text { OK Update } \\
\text { 15:51:53 Send Packets } \\
\text { 15:52:37 Break } \\
15: 52.37 \text { Tr Session End } \\
\text { 15:52:54 Send Packets }\end{array}$} & \multirow{8}{*}{ 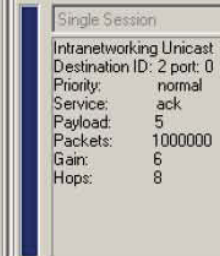 } & \multirow{6}{*}{$\begin{array}{ll}\text { Accepted } & 2452 \\
\text { Success } & 2266 \\
\text { No Ack } & 0 \\
\text { Blocked } & 167 \\
\text { Timeout } & 6 \\
\text { Entor } & 0 \\
\text { Routed } & 2433 \\
\text { Rx Failed } & 0 \\
\text { Total Tx } & 2305 \\
\end{array}$} & \multirow{4}{*}{$\begin{array}{l}\text { Total } \\
\text { Mine } \\
\text { Other } \\
\text { Bad } \\
\text { Bit Rate }\end{array}$} & \multirow{4}{*}{$\begin{array}{l}580 \\
580 \\
0 \\
0 \\
0.52 \\
\end{array}$} \\
\hline & & & & & & \\
\hline & & & & & & \\
\hline & & & & & & \\
\hline & & & & & \multicolumn{2}{|c|}{ Routed Statistics } \\
\hline & & & & & Routed & 0 \\
\hline & & & & & $\begin{array}{l}\text { success } \\
\text { no ack }\end{array}$ & $0_{0}^{0}$ \\
\hline & & $\mathrm{T}_{\mathrm{E}} / \mathrm{Rx}$ & & & $\begin{array}{l}\text { blocked } \\
\text { rx faled } \\
\text { no hops }\end{array}$ & $\begin{array}{l}0 \\
0 \\
0\end{array}$ \\
\hline
\end{tabular}


EMC Test Setup \& Operation for PLC Communication

Released $7-28$ - 2009

Document Rev 1.0

Phase I: Vitron AC Setup and Test Continued:

Step 9 Observation: Observe activity occurs on both Vitron Unit $1 \& 2$. See photos below for an illustration of transmit and receive activity.

Vitron \#1

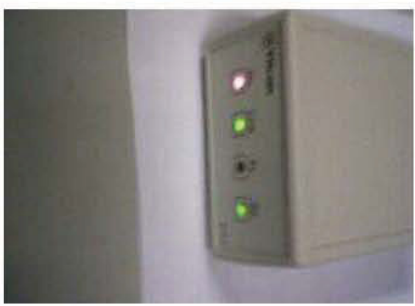

Vitron \#2

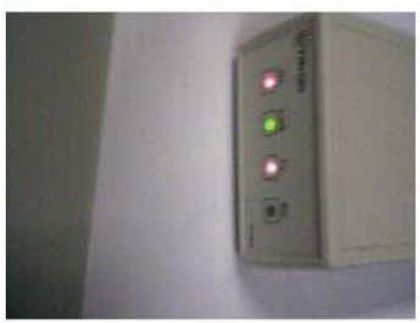

Summary: From this point on the devices are operating, EMC test measurements can now take place as called for on the test specification in the PO. When measurements are done, tear down the test setup by clicking exit under the General menu heading. Turn off the laptop, Vitron devices and then remove and place in a box ready for shipment back to eTec.

This Concludes Phase I 
EMC Test Setup \& Operation for PLC Communication

Released $7-28$ - 2009

Document Rev 1.0

Phase II: Vitron DC Setup and Test Continued:

Required Material: The following materials will be needed for setting up Phase II

- Two Vitron Units labeled 1,2

- One USB Cable

- Two DC Power Supplies for the Vitron Units.

- One Laptop Computer with Vitron Software

Procedure: Apply the following steps in setting up and operating the Vitron units in DC mode using the following steps and accompanying diagrams and drawings.

\section{Hardware Setup}

Step 1: Using Figure 2.0 located on page 1 of 2 on the drawing titled, "EMC Test Layout" as a reference connect Vitron 1 to the test laptop using a USB cable.

Step 2: Connect Vitron 1 to its power adapter and plug the power adapter into a DC Power Supply check the LED light illuminates before preceeding further, if it fails to light up, the battery needs to be recharged and testing will need to take place the following day.

Step 3: Connect Vitron 2 to its power adapter and plug it into another DC power supply. Again if the LED fails to illuminate, the DC power supply needs to recharge.

Observes Vitron 2 does not have a USB cable connected to the laptop. This is a deliberate action given the interest in measuring PLC communication exclusively.

This completes setting up the hardware for this Phase.

Test Operation Section: Continue with steps $4-9$ as described in Phase I.

\section{This Concludes Phase II}


EMC Test Setup \& Operation for PLC Communication

Phase III: Ethernet Intellon Device

Required Material: The following materials will be needed for setting up Phase III

- Two Intellon AV Ethernet devices

- Two Ethernet Cables

- Two Laptops.

Procedure: Apply the following steps in setting up and operating the Homeplug AV Ethernet devices.

\section{Hardware Setup}

Step 1: Using Figure 3.0 located on page 2 of 2 on the drawing titled, "EMC_Test Layout". Plug in each device into two separate receptacles.

Step 2: Connect an Ethernet cable from laptop 1 to Intellon Ethernet device \#1.

Step 3: Repeat Step 2 for Laptop 2 and Intellon AV Ethernet device \#2.

This Completes setting up the hardware for this phase.

\section{Software Setup}

Step 4: Turn on test laptop $1 \& 2$ and assign the following

$$
\begin{array}{r}
\text { Laptop \#1 IP Address }(\text { static })=192.168 .0 .100 \\
\text { Default Gateway }=192.168 .0 .100 \\
\text { Subnet Mask }=255.255 .255 .0 \\
\text { Laptop \#2 IP Address(static) }=192.168 .0 .101 \\
\text { Default Gateway }=192.168 .0 .101 \\
\text { Subnet Mask }=255.255 .255 .0 \\
\{\text { Keep the workgroup. }\}
\end{array}
$$

Step 5: Transfer a large file from Laptop 1 to Laptop 2. Conduct EMC measurements during the file transfer. Use the CAD Video File.

\section{This Concludes Phase III}


EMC Test Setup \& Operation for PLC Communication

Phase IV: Maxim 2990 Test setup \& Operation:

Required Material: The following materials will be needed for setting up Phase IV

- Two Maxim 2990 devices

- Two Serial Cables

- Two Laptops.

- Power Accessories Kit

- Serial to Fiber converters

Procedure: Apply the following steps in setting up and operating the Maxim 2990 devices for 110 Vac test operation.

\section{Hardware Setup}

Step 1: Examine Figure 6.0 located on page 1 of 2 on the drawing titled, "EMC Maxim 2990". Using modules contained the power accessories kit, create two $110 \mathrm{Vac}$ plugs.

Step 2: Connect the cold wires to DUT \#1 and DUT $\# 2$ on as illustrated in Figure 6.0. Use the overall layout snapshot located below figure 6.0 as a guide.

Step 3: Connect the cold wires by matching the correct polarity with jumper wires colored as red $=+$ and Black $=-$.

Step 4: Examine the LED flash rate between DUT\#1 and Dut $\# 2$. There should be two flashing lights operating in an alternating sequence. This indicates there is packet data being transferred between DUT\#1 and DUT\#2 .

Step 5: Connect both laptops\#1\&2 to DUT\#1\&2 respectively, using a serial cable as See figure 6.0 on the "EMC Maxim 2990" drawing. 
EMC Test Setup \& Operation for PLC Communication

Released 7-28 - 2009

Document Rev 1.0

\section{Software Setup}

Step 6: Turn on laptop 1 \& 2 and select the program icon " $t 134$ mpro" on both laptops see illustration below:

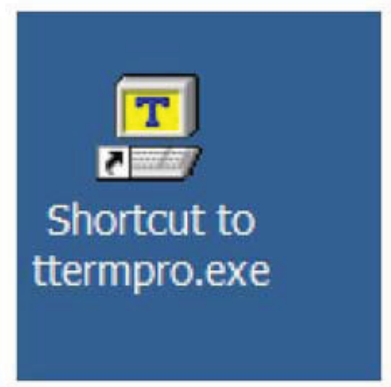

Step 7: After selecting the icon, the following dialogue box appears illustrating packet data transfer between DUT \#1 and DUT\#2

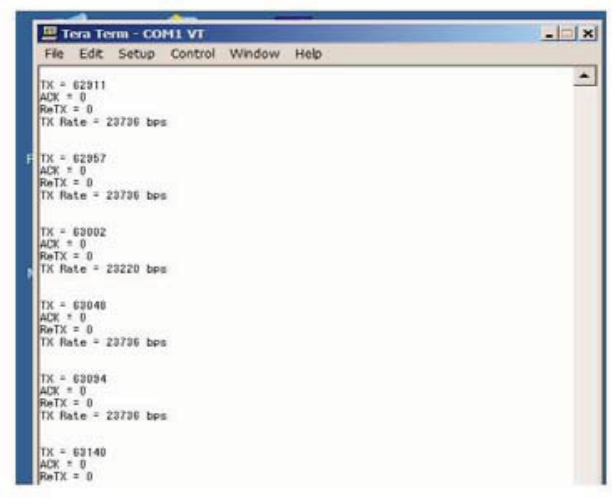

Step 8: Verify traffic data is real by disconnecting one of the cold wires and observing all traffic activity STOPS between both laptops. 
EMC Test Setup \& Operation for PLC Communication

\section{This Concludes Phase IV}

Phase V: Maxim 2990 Test setup \& Operation 12V dc:

Required Material: The following materials will be needed for setting up Phase V

- Two Maxim 2990 devices

- Two Serial Cables

- Two Laptops.

- Power Accessories Kit

- Serial to Fiber converters

- $12 \mathrm{~V}$ de Power Supply

Procedure: Apply the following steps in setting up and operating the Maxim 2990 devices for 12 Vdc test operation.

\section{Hardware Setup}

Step 1: Examine Figure 6.0 located on page 1 of 2 on the drawing titled, "EMC Maxim 2990". Using modules contained the power accessories kit, select the 100 to $200 \mathrm{Vac}$ input to $12 \mathrm{Vdc}$ output power adapter, create the plug for either $110 \mathrm{Vac}$ or a $220 \mathrm{Vac}$ receptacle.

Step 2: Connect the other end into the $12 \mathrm{Vdc}$ receptacle on each DUT.

Step 3: Connect the cold wires to DUT\#1 and DUT\#2 on as illustrated in Figure 6.0. Use the overall layout snapshot located below figure 6.0 as a guide.

Step 4: Connect the cold wires by matching the correct polarity with jumper wires colored as red $=+$ and Black $=-$.

Step 5: Examine the LED flash rate between DUT\#1 and Dut $\# 2$. There should be two flashing lights operating in an alternating sequence. This indicates there is packet data being transferred between DUT\#1 and DUT\#2.

Step 6: Connect both laptops\#1\&2 to DUT\#1\&2 respectively, using a serial cable as See figure 6.0 on the "EMC Maxim 2990" drawing. 
EMC Test Setup \& Operation for PLC Communication

Released $7-28$ - 2009

Document Rev 1.0

\section{Software Setup}

Step 6: Turn on laptop $1 \& 2$ and select the program icon "tt34mpro" on both laptops see illustration below:

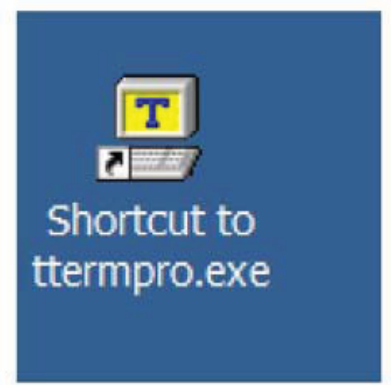

Step 7: After selecting the icon, the following dialogue box appears illustrating packet data transfer between DUT \#1 and DUT\#2

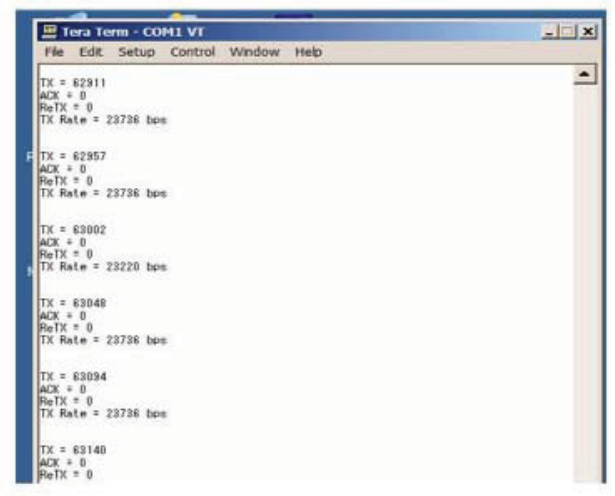

Step 8: Verify traffic data is real by disconnecting one of the cold wires and observing all traffic activity STOPS between both laptops.

\section{This Concludes Phase V}


EMC Test Setup \& Operation for PLC Communication

Released $7-28-2009$

Document Rev 1.0

This Concludes Test Setup and Operation Procedure for PLC Communications. 


\section{Appendix B-6 \\ Electromagnetic Capability Test Setup Drawings at Electric Transportation Applications Engineering Center}
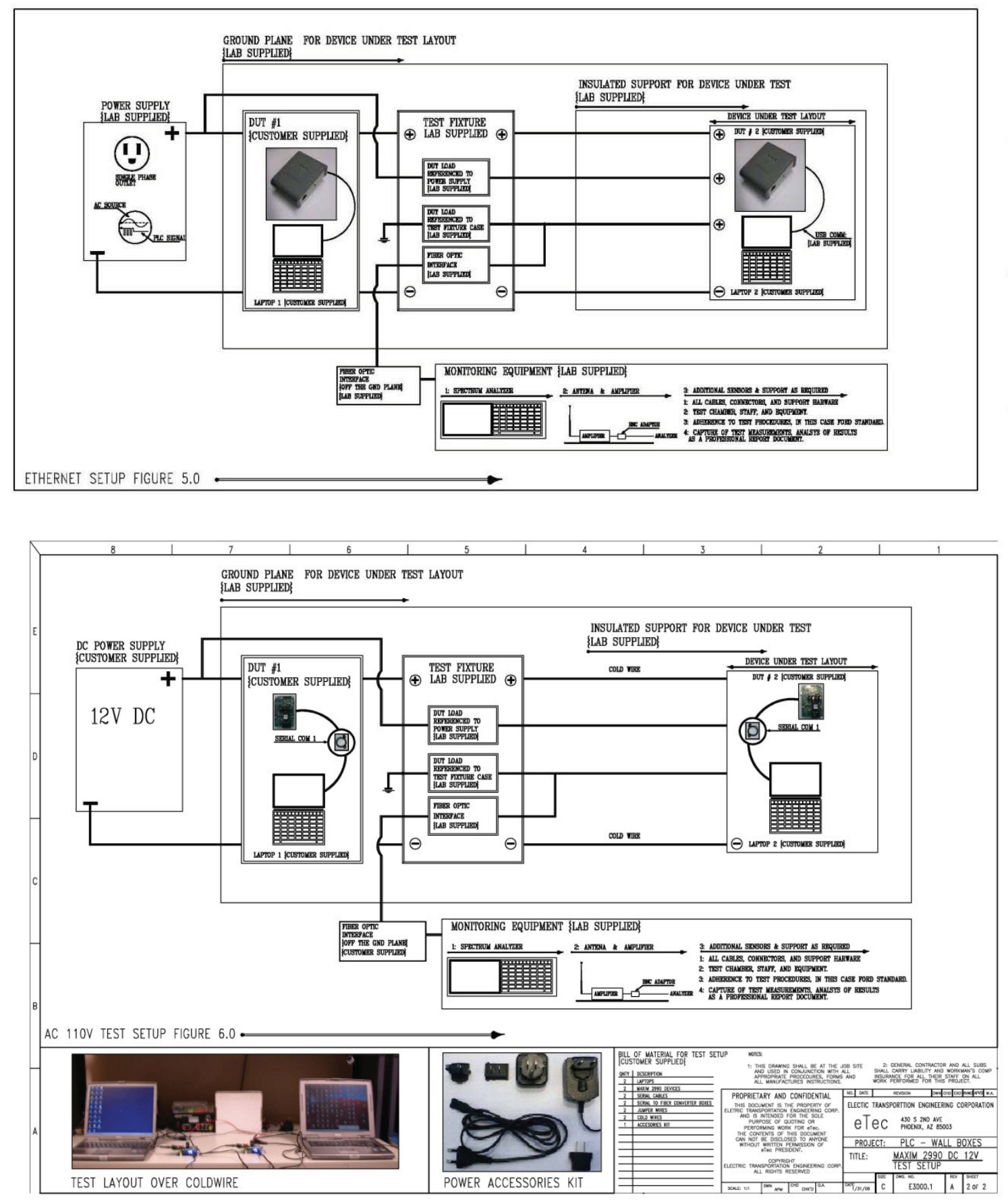


\section{Appendix B-7 \\ Electromagnetic Capability Test Report (CPK - Maxim 2990/2991)}

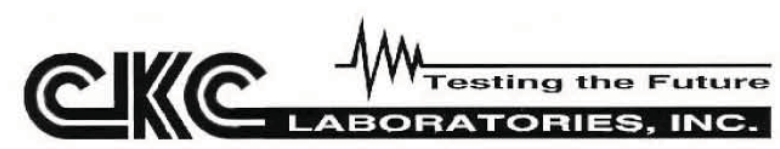

CKC Laboratories, Inc

$\begin{aligned} & \text { Customer: } \\
& \text { Specification: } \\
& \text { RE310-30-1000MHz-Level 1-Limit A-Peak ES-XW7T-1A278-AC Date Issued: October } 102003\end{aligned}$
\begin{tabular}{|l|l|l|l|}
\hline Work Order \#: & 90010 & Date: & Sat Sep-12-2009 \\
\hline Test Type: & Radiated Scan & Time: & $1: 05.51$ PM \\
\hline Equipment: & $\begin{array}{l}\text { Power Line Communications Devices With } \\
\text { G3 AFE }\end{array}$ & Sequence: & 9 \\
\hline Manufacturer: & Maxim Integrated Products & Tested By: & A. Brar \\
\hline Model: & MAX2991 EV Kit FCC High Power Rev 1 & & \\
\hline S/N: & None & File Name: & $\begin{array}{l}\text { RE SEQ9 30-242.4MHZ-HORIZ-FCC } \\
\text { HP.DAT }\end{array}$ \\
\hline
\end{tabular}

\begin{tabular}{|c|c|c|c|c|}
\hline Equipment & $S / N$ & Calibrated Date & Cal Due Date & Asset\# \\
\hline Cable-12' Pasternack & None & $12 / 11 / 2007$ & $12 / 11 / 2009$ & P05438 \\
\hline $\begin{array}{l}\text { LISN 5uH - 9117-5-TS-50- } \\
\text { N }\end{array}$ & 46583 & $03 / 31 / 2009$ & $03 / 31 / 2011$ & 2644 \\
\hline $\begin{array}{l}5 \mathrm{UH} \text { LISN 9117-5-TS-50- } \\
\mathrm{N}\end{array}$ & 46584 & $10 / 02 / 2008$ & $10 / 02 / 2010$ & 2645 \\
\hline $\begin{array}{l}\text { LISN } 5 u H-9117-5-T S-50- \\
N\end{array}$ & 68844 & $11 / 07 / 2007$ & $11 / 07 / 2009$ & 2860 \\
\hline $\begin{array}{l}\text { LISN } 5 \mathrm{UH}-9117-5-\mathrm{TS}-50- \\
N\end{array}$ & 089466 & $07 / 07 / 2009$ & $07 / 07 / 2011$ & 03153 \\
\hline 10uF Cap 6512-106R & 963816 & $11 / 01 / 2007$ & $11 / 01 / 2009$ & P01656 \\
\hline 10uF Cap 6512-106R & 583 & $11 / 01 / 2007$ & $11 / 01 / 2009$ & 583 \\
\hline 10uF Cap 6512-106R & $\times 05$ & $11 / 07 / 2007$ & $11 / 07 / 2009$ & 142 \\
\hline 10uF Cap 6512-106R & 87115 & $11 / 01 / 2007$ & $11 / 01 / 2009$ & 1729 \\
\hline 50UH LISN & 17512 & $01 / 27 / 2009$ & $01 / 27 / 2010$ & 2527 \\
\hline $50 \mathrm{UH}$ LISN & 17510 & $01 / 27 / 2009$ & $01 / 27 / 2010$ & 2532 \\
\hline SA-E4446A & US44300408 & $03 / 09 / 2009$ & $03 / 09 / 2011$ & 2668 \\
\hline Cable-RG214/U & None & $03 / 06 / 2009$ & $03 / 06 / 2011$ & P05297 \\
\hline Andrew Cable- CNT-195 & none & $12 / 11 / 2008$ & $12 / 11 / 2010$ & P01184 \\
\hline $\begin{array}{l}\text { Bi-Con Antenna SAS- } \\
200 / 540\end{array}$ & 359 & $11 / 03 / 2008$ & $11 / 03 / 2010$ & 46 \\
\hline HP 8447D Preamp & 2944A03850 & $01 / 06 / 2009$ & $01 / 06 / 2011$ & 501 \\
\hline
\end{tabular}

Equipment Under Test $\left(^{*}=\right.$ EUT):

\begin{tabular}{|c|c|c|c|}
\hline Function & Manufacturer & Model\# & $S / N$ \\
\hline $\begin{array}{l}\text { "Power Line Communications } \\
\text { Devices With G3 AFE }\end{array}$ & Maxim Integrated Products & $\begin{array}{l}\text { MAX2991 EV Kit FCC High } \\
\text { Power Rev } 1\end{array}$ & None \\
\hline \multicolumn{4}{|l|}{ Support Devices: } \\
\hline Function & Manufacturer & Model\# & $S / N$ \\
\hline None & & & \\
\hline
\end{tabular}

None

Test Conditions / Notes:

The EUT under test is setup on $5 \mathrm{~cm}$ non conductive surface. The AC power and DC power leads are routed $10 \mathrm{~cm}$ in from the edge of the test table to the LISNs. The two sets of DC powers are routed to the four $5 \mathrm{UH}$ LISNs located at the edge of the test table, the power input of these LISNS is connected to two 10uF Feed-Thru capacitors mounted on the wall between the ante-room and test chamber On the other side in the ante-room these two capacitors are connected to a DC power supply's output The two AC powers over which the EUT communicates are connected to two 50 UH LISNs due to impedance issue and the EUT not communicating. The input of these two 50uH LISNs is connected to two 10uF Feed-Thru capacitors mounted on the wall between the test chamber and Ante-Room, the other side of the capacitors is plugged into $110 \mathrm{~V} / 6 \mathrm{OHz}$ power. EUT's are communicating over the AC power connected to the two $50 \mathrm{uH}$ LISNS

Transducer Legend: T1=Cable ANP05438

T3=CAB-ANP01184-121108 T2=CAB-ANP05297-030609

Measurement Data:
\begin{tabular}{|r|r|r|r|r|r|r|r|r|r|r|r|r|r|r|}
\hline$\#$ & Freq MHz & $\begin{array}{r}\text { Rdng } \\
\mathrm{dB} \mu \mathrm{V}\end{array}$ & $\begin{array}{r}\mathrm{T} 1 \\
\mathrm{~T} 5\end{array}$ & $\mathrm{~T} 2$ & $\mathrm{~T} 3$ & $\mathrm{~T} 4$ & Dist & $\begin{array}{r}\text { Corr } \\
\mathrm{dB} \mu \mathrm{V} / \mathrm{m}\end{array}$ & $\begin{array}{r}\text { Spec } \\
\mathrm{dB} \mu \mathrm{V} / \mathrm{m}\end{array}$ & & & Polar & Type & Margin \\
\hline 1 & 96.006 & 32.1 & $\begin{array}{r}+0.4 \\
+10.9\end{array}$ & +0.7 & +0.1 & -26.5 & +0.0 & 17.7 & 43.6 & & & Horiz & Peak & $\mathbf{- 2 5 . 9}$ \\
\hline 2 & 192.012 & 27.4 & $\begin{array}{r}+0.5 \\
+16.2\end{array}$ & +0.9 & +0.3 & -26.2 & +0.0 & 19.1 & 48.2 & & & Horiz & Peak & $\mathbf{- 2 9 . 1}$ \\
\hline
\end{tabular}




\begin{tabular}{|c|c|c|c|c|c|c|c|c|c|c|c|c|}
\hline & & & & & & & & & & \multicolumn{3}{|c|}{$\begin{array}{l}\text { CKC Laboratories, Inc. } \\
1120 \text { Fulton Place. } \\
\text { Fremont. CA 94539 } \\
(510) 249-1170\end{array}$} \\
\hline \# & Freq $\mathrm{MHz}$ & $\begin{array}{l}\text { Rdng } \\
\text { dBuV }\end{array}$ & $\begin{array}{l}\text { T1 } \\
\text { T5 }\end{array}$ & T2 & T3 & T4 & Dist & $\begin{array}{r}\text { Corr } \\
d B \mu V / m\end{array}$ & $\begin{array}{r}\text { Spec } \\
d B \mu V / m\end{array}$ & Polar & Type & Margin \\
\hline 3 & 211.211 & 28.2 & $\begin{array}{r}+0.6 \\
+15.1\end{array}$ & +0.9 & +0.4 & -26.0 & +0.0 & 19.2 & 48.8 & Horiz & Peak & -29.6 \\
\hline 4 & 57.608 & 29.5 & $\begin{array}{r}+0.4 \\
+11.1\end{array}$ & +0.5 & +0.2 & -26.6 & +0.0 & 15.1 & 44.9 & Horiz & Peak & -29.8 \\
\hline 5 & 76.807 & 26.1 & $\begin{array}{r}+0.4 \\
+10.8\end{array}$ & +0.6 & +0.1 & -26.6 & +0.0 & 11.4 & 42.2 & Horiz & Peak & -30.8 \\
\hline 6 & 134.404 & 25.0 & $\begin{array}{r}+0.5 \\
+14.2\end{array}$ & +0.8 & +0.2 & -26.4 & +0.0 & 14.3 & 45.8 & Horiz & Peak & -31.5 \\
\hline 7 & 153.614 & 26.1 & $\begin{array}{r}+0.4 \\
+13.7\end{array}$ & +0.8 & +0.2 & -26.3 & +0.0 & 14.9 & 46.7 & Horiz & Peak & -31.8 \\
\hline 8 & 104.404 & 25.5 & $\begin{array}{r}+0.4 \\
+11.7\end{array}$ & +0.7 & +0.2 & -26.5 & +0.0 & 12.0 & 44.2 & Horiz & Peak & -32.2 \\
\hline 9 & 102.002 & 25.2 & $\begin{array}{r}+0.4 \\
+11.4\end{array}$ & +0.7 & +0.2 & -26.5 & +0.0 & 11.4 & 44.0 & Horiz & Peak & -32.6 \\
\hline 10 & 38.398 & 29.9 & $\begin{array}{r}+0.4 \\
+12.1 \\
\end{array}$ & +0.5 & +0.2 & -26.7 & +0.0 & 16.4 & 49.3 & Horiz & Peak & -32.9 \\
\hline
\end{tabular}

C:LOCUME -1VIM 1. LECLLOCALS 1ITempITemporary Directory 10 for RE SEQ1-40.zipIRE SEQ9 30-242.4MHz-Horiz-FCC HPFage 2 of 3 
CKC Laboratories, Inc

1120 Fulton Place.

Fremont. CA 94539

(510) $249-1170$
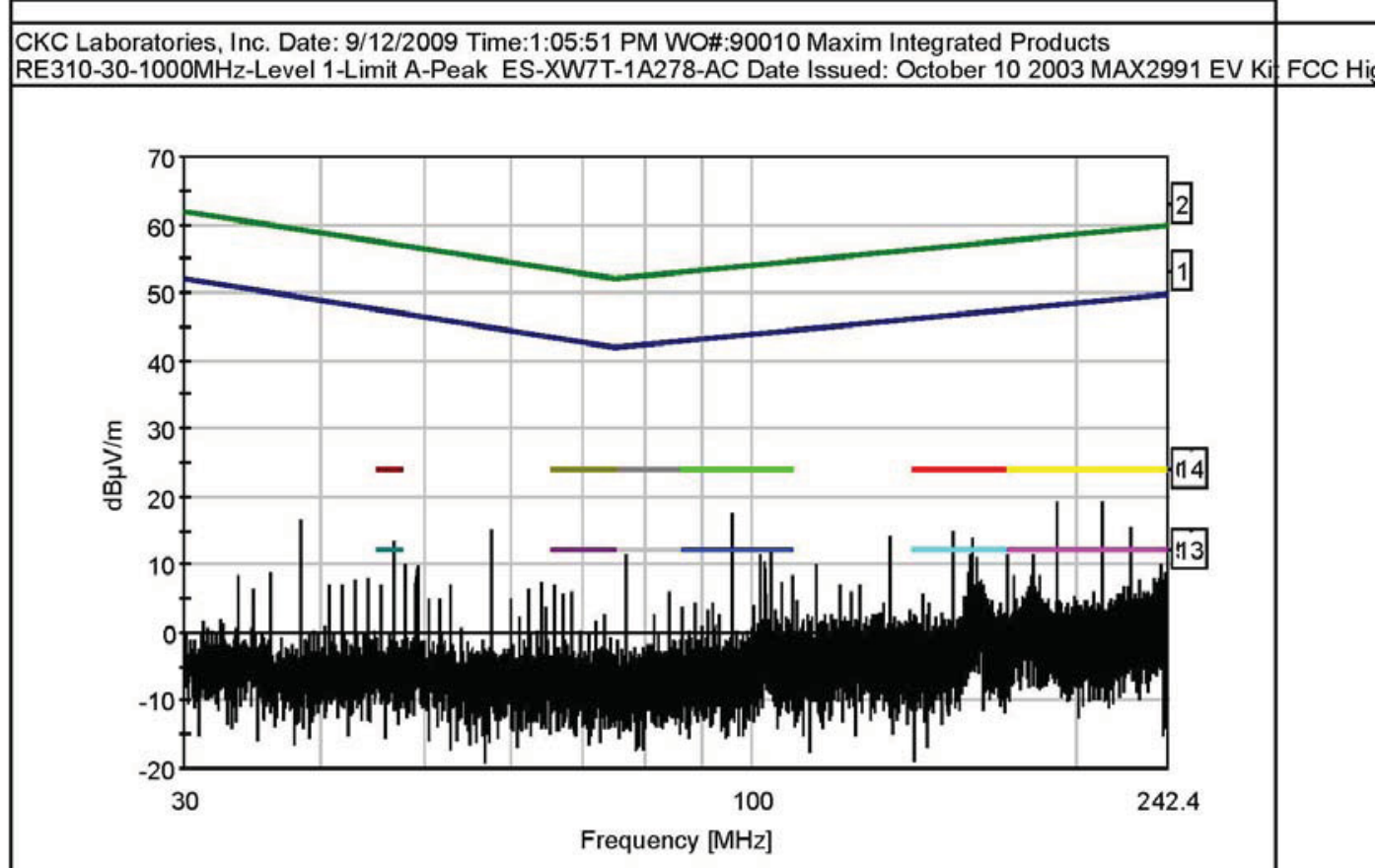


\section{Appendix B-8 \\ Electromagnetic Capability Test Report (Compliance Testing)}

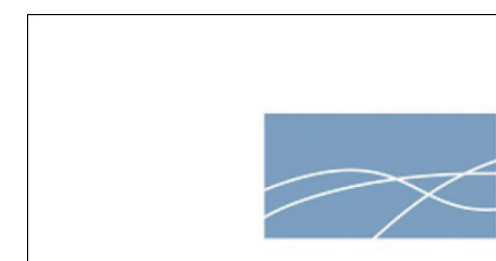

\section{Compliance Testing}

Previously Flom Test lab

RF, EMC \& Safety Testing Experts Since 1963

loll-free: ( 866$) 311-3268$

$\operatorname{fax}:(480) 926-3598$

Irtp//www. ComplarreTetirg. com

\section{Engineering Development EMC/EMI Test Report}

for

Models: Netgear XAV101, Yitran IT700, and Maxim 2990 EV Kit

to

FCC Part 15, B Unintentional Radiator Class B

and

Ford ES-XW7T-1A278-AC:2003 (ED- Engineering Development)

RE310- Radiated Emissions

CE420- Conducted Emissions

RI112- Bulk Current Injection (BCl)

RI114- Radiated RF Immunity

Date of Report: October 16, 2009

At the Request of:

eTec

$430 \mathrm{~S}$ 2nd Ave

Phoenix, Az 85003

Attention of:

Andre Masters

Ph: (602) 716-9576 Ext 23

E-mail: amasters@etecevs.com

Reviewed By:

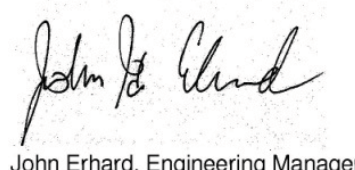

John Erhard, Engineering Manager

Compliance Testing

3356 N. San Marcos Place, Suite 107

Chandler, Arizona 85225-7176

(866) 311-3268 phone, (480) 926-3598 fax

p09a0005, d09a0009 Rev 6.0 
Compliance Testing

Test Report Revision History

\begin{tabular}{|l|l|l|l|}
\hline Revision & Date & Revised By & Reason for revision \\
\hline 1.0 & October 16,2009 & S Nickels & Original Document \\
\hline 2.0 & October 23,2009 & S. Nickels & Model \#'s added \\
\hline 3.0 & October 26, 2009 & S. Nickels & Added CE correction \\
\hline 4.0 & October 26, 2009 & S. Nickels & Added CE "Line" correction \\
\hline 5.0 & October 26,2009 & S. Nickels & Add Broadband over Power-line comment. \\
\hline 6.0 & October 26, 2009 & S. Nickels & Add Ford-AC CE Limit. Statement. \\
\hline
\end{tabular}

Compliance Testing

3356 N. San Marcos Place, Suite 107

Chandler, Arizona 85225-7176

(866) 311-3268 phone, (480) 926-3598 fax

p09a0005, d09a0009 Rev 6.0 


\section{Compliance Testing}

\section{Table Of Contents}

Rule

Description

Page

Test Report

Standard Test Conditions and Engineering Practices

Test Results Summary

Radiated Emissions

Radiated Emissions

Powerline Conducted Emissions

Radiated Immunity Bulk Current Injection

Radiated Immunity- ALSE Method

Test Equip Utilized

1
2
3
4
1
4
2
26
8

Compliance Testing

3356 N. San Marcos Place, Suite 107

Chandler, Arizona 85225-7176

(866) 311-3268 phone, (480) 926-3598 fax

p09a0005, d09a0009 Rev 6.0 
a)

b) Laboratory: (FCC: 31040/SIT)

(Canada: IC 2044A-1

c) Report Number:

d) Client:

e) Identification:

Description:

f) EUT Condition

g) Report Date:

$h, j, k)$ :

i) Sampling method:

I) Uncertainty:

m) Supervised by:

n) Results:

o) Reproduction:

\section{Test Report}

Compliance Testing

3356 N. San Marcos Place, Suite 107

Chandler, AZ 85225

d09a0009

eTec

Netgear XAV101, Yitran IT700, and Maxim 2990 EV Kit

PLC Power Line Com System

Not required unless specified in individual tests.

October 16, 2009

As indicated in individual tests.

No sampling procedure used.

In accordance with Compliance Testing internal quality manual

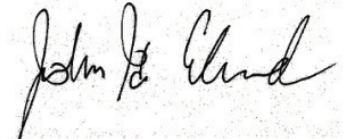

John Erhard, Engineering Manager

The results presented in this report relate only to the item tested.

This report must not be reproduced, except in full, without written permission from this laboratory.

Compliance Testing

3356 N. San Marcos Place, Suite 107

Chandler, Arizona 85225-7176

(866) 311-3268 phone, (480) 926-3598 fax 


\section{Test And Measurement Data}

All tests and measurement data shown were performed in accordance with FCC Rules and Regulations, Volume II; Part 2 and the following individual Parts: 15.107, 15.109; Unintentional Radiators. Ford-AC testing was within the guidelines of the ES-XW7T-1A278-AC (October 2003) specification and the testing requests of the client.

All Conducted Emissions testing was done to FCC Limits, due to the Ford-AC limits being significantly higher.

\section{Standard Test Conditions and Engineering Practices}

Except as noted herein, the following conditions and procedures were observed during the testing:

In accordance with ANSI C63.4-2003, and unless otherwise indicated in the specific measurement results, the ambient temperature of the actual EUT was maintained within the range of $10^{\circ}$ to $40^{\circ} \mathrm{C}\left(50^{\circ}\right.$ to $\left.104{ }^{\circ} \mathrm{F}\right)$ unless the particular equipment requirements specify testing over a different temperature range. Also, unless otherwise indicated, the humidity levels were in the range of $10 \%$ to $90 \%$ relative humidity.

Prior to testing, the EUT was tuned up in accordance with the manufacturer's alignment procedures. All external gain controls were maintained at the position of maximum and/or optimum gain throughout the testing.

Measurement results, unless otherwise noted, are worst-case measurements.

FCC OATS Reg. \#933597

IC O.A.T.S. Number: 2044 A-1

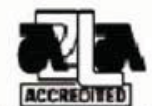

TESTIIIG CERT $\# 2152.01$

This device is subject to Broadband over Power Line testing as defined in FCC Part 15 Rules, subpart G.

This report does not reflect this testing because it was not evaluated at this time.

Compliance Testing

3356 N. San Marcos Place, Suite 107

Chandler, Arizona 85225-7176

(866) 311-3268 phone, (480) 926-3598 fax

Page 2 of 28

p09a0005, d09a0009 Rev 6.0 
Test Results Summary:

Netgear XAV101

\begin{tabular}{|c|c|c|c|}
\hline Specification & Test Name & Pass, Fail, N/A & Comments \\
\hline FCC 15.109 & Radiated Emissions & Fail & $\begin{array}{c}\text { Netgear XAV101 was over limit at 500MHz } \\
\text { (see data) }\end{array}$ \\
\hline Ford-AC & RE310 Radiated Emissions & Pass & Netgear XAV101 over limit 150kHz-30MHz \\
\hline FCC 15.107 & Power line Conducted Emissions & Fail & \\
\hline Ford-AC & CE420 Conducted Emissions & Pass & \\
\hline Ford-AC & Rl112 Bulk Current Injection (BCl) & Pass & \\
\hline Ford-AC & Rl114 Radiated Immunity (ALSE) & Pass & \\
\hline
\end{tabular}

Yitran $1 T 700$

\begin{tabular}{|c|c|c|c|}
\hline Specification & Test Name & Pass, Fail, N/A & Comments \\
\hline FCC 15.109 & Radiated Emissions & Pass & \\
\hline Ford-AC & RE310 Radiated Emissions & Pass & \\
\hline FCC 15.107 & Power line Conducted Emissions & Pass & \\
\hline Ford-AC* & CE420 Conducted Emissions & Pass & \\
\hline Ford-AC & RI112 Bulk Current Injection (BCI) & Pass & \\
\hline Ford-AC & Rl114 Radiated Immunity (ALSE) & Pass & \\
\hline
\end{tabular}

\section{Maxim 2990 EV Kit}

\begin{tabular}{|c|c|c|c|}
\hline Specification & Test Name & Pass, Fail, N/A & Comments \\
\hline FCC 15.109 & Radiated Emissions & Pass & \\
\hline Ford-AC & RE310 Radiated Emissions & Pass & \\
\hline FCC 15.107 & Power line Conducted Emissions & Pass & \\
\hline Ford-AC & CE420 Conducted Emissions & Pass & \\
\hline Ford-AC & Rl112 Bulk Current Injection (BCl) & Pass & \\
\hline Ford-AC & Rl114 Radiated Immunity (ALSE) & Pass & \\
\hline
\end{tabular}

* Ford-AC Conducted Emissions was only tested to EU1 and G1 limits due to the very high limit line as compared to the FCC Conducted Emissions testing. All 3 test samples passes the Ford-AC Conducted Emission requirement for ranges EU1 and G1.

Compliance Testing

3356 N. San Marcos Place, Suite 107

Chandler, Arizona 85225-7176

(866) 311-3268 phone, (480) 926-3598 fax

Page 3 of 28

p09a0005, d09a0009 Rev 6.0 
Name of Test:

Specification:

Test Equipment Utilized
Radiated Emissions

15.109

i00033, i00267
Engineer: Sam Nickels, NCT

Test Date: October 16, 2009

\section{Test Procedure}

The EUT was tested in an Open Area Test Site (OATS) set $3 \mathrm{~m}$ from the receiving antenna. An EMI Receiver was used to verify that the EUT met the requirements for Radiated Emissions. The EUT was tested by rotating it $360^{\circ}$ with the antennas in both the vertical and horizontal orientation and raised from 1 to 4 meters to ensure the TX signal levels were maximized. All emissions from $30 \mathrm{MHz}$ to $1 \mathrm{GHz}$ were examined.

\section{Test Setup}

\begin{tabular}{|l|l}
\hline EUT & Antenna \\
\end{tabular}

Compliance Testing

3356 N. San Marcos Place, Suite 107

Chandler, Arizona 85225-7176

(866) 311-3268 phone, (480) 926-3598 fax

Page 4 of 28

p09a0005, d09a0009 Rev 6.0 

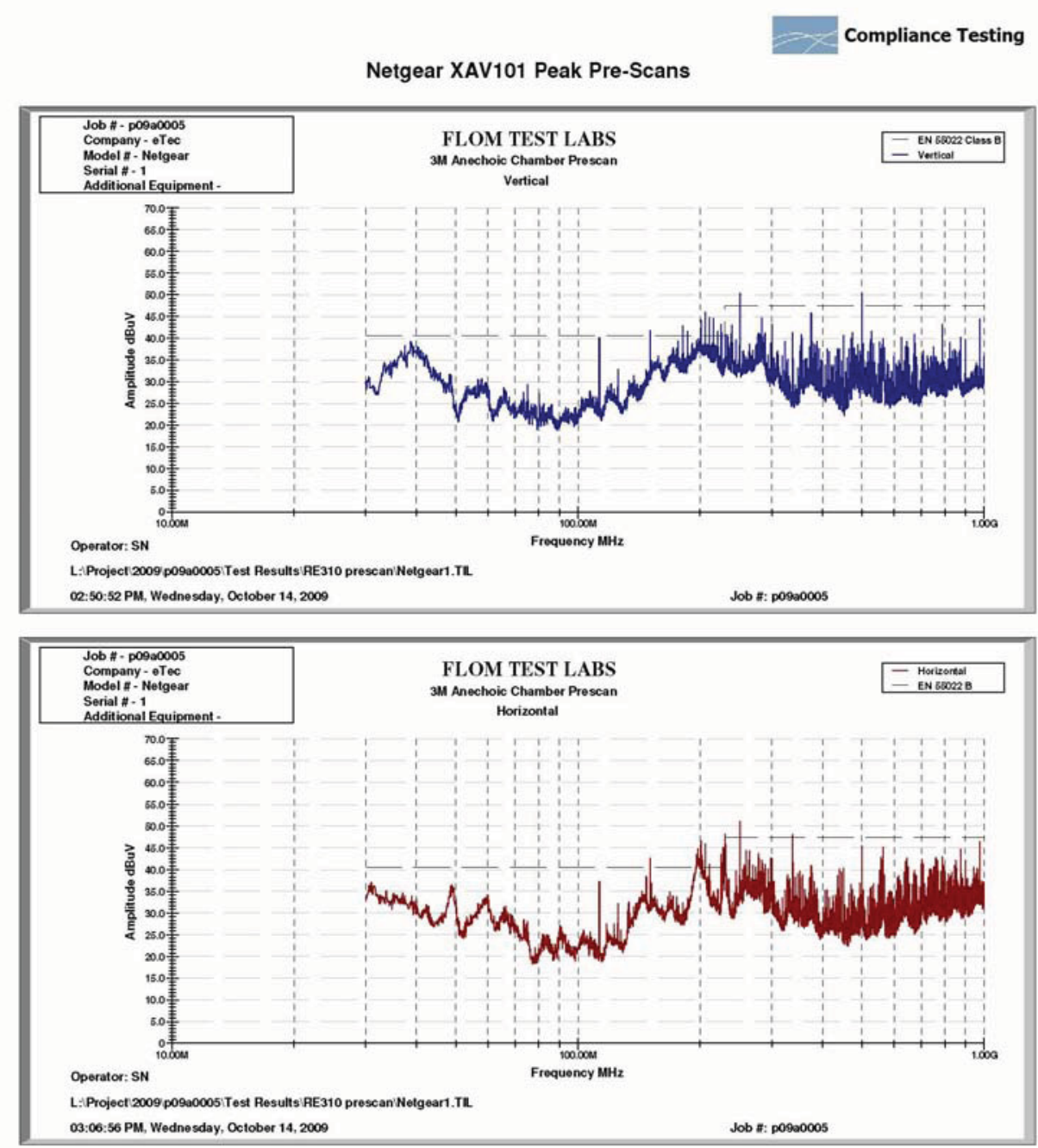

Quasi-peak Radiated Emissions-

\begin{tabular}{|c|c|c|c|c|c|}
\hline $\begin{array}{c}\text { Emission Freq } \\
(\mathrm{MHz})\end{array}$ & $\begin{array}{c}\text { Measured Value } \\
(\mathrm{dBuV} / \mathrm{m})\end{array}$ & $\begin{array}{c}\text { Correction Factor } \\
(\mathrm{dB})\end{array}$ & $\begin{array}{c}\text { Corrected Value } \\
(\mathrm{dBuV} / \mathrm{m})\end{array}$ & $\begin{array}{c}\text { Limit } \\
(\mathrm{dBuV} / \mathrm{m})\end{array}$ & $\begin{array}{c}\text { Margin } \\
\mathrm{dB}\end{array}$ \\
\hline $40.27(\mathrm{~V})$ & 15.7 & 13.6 & 29.2 & 40.0 & -10.8 \\
\hline $40.27(\mathrm{H})$ & 1.4 & 15.1 & 16.5 & 40.0 & -23.5 \\
\hline $112.51(\mathrm{~V})$ & 16.1 & 12.9 & 29.0 & 43.0 & -14.0 \\
\hline $375.00(\mathrm{~V})$ & 27.4 & 18.0 & 45.4 & 46.0 & -0.6 \\
\hline $375.00(\mathrm{H})$ & 27.1 & 18.0 & 45.1 & 46.0 & -0.9 \\
\hline $500.00(\mathrm{~V})$ & 27.9 & 20.6 & 48.5 & 46.0 & $+\mathbf{2 . 5}$ \\
\hline
\end{tabular}

Compliance Testing

3356 N. San Marcos Place, Suite 107

Chandler, Arizona 85225-7176

(866) 311-3268 phone, (480) 926-3598 fax

Page 5 of 28

p09a0005, d09a0009 Rev 6.0 
Netgear XAV101 Radiated Emissions Test Setup Photos:
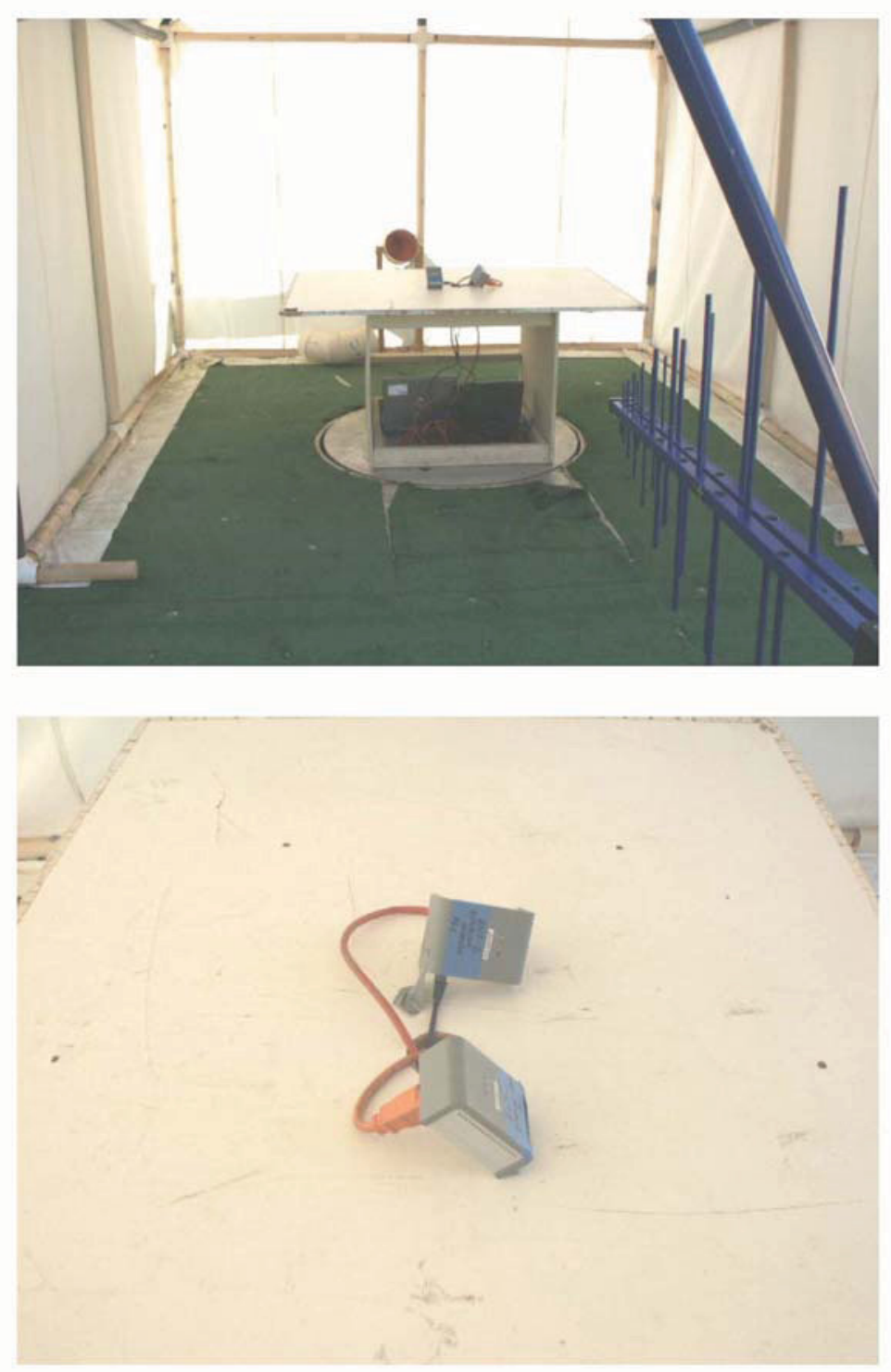

Compliance Testing

3356 N. San Marcos Place, Suite 107

Chandler, Arizona 85225-7176

(866) 311-3268 phone, (480) 926-3598 fax

Page 6 of 28 p09a0005, d09a0009 Rev 6.0 
Yitran IT700 Peak Pre-Scans
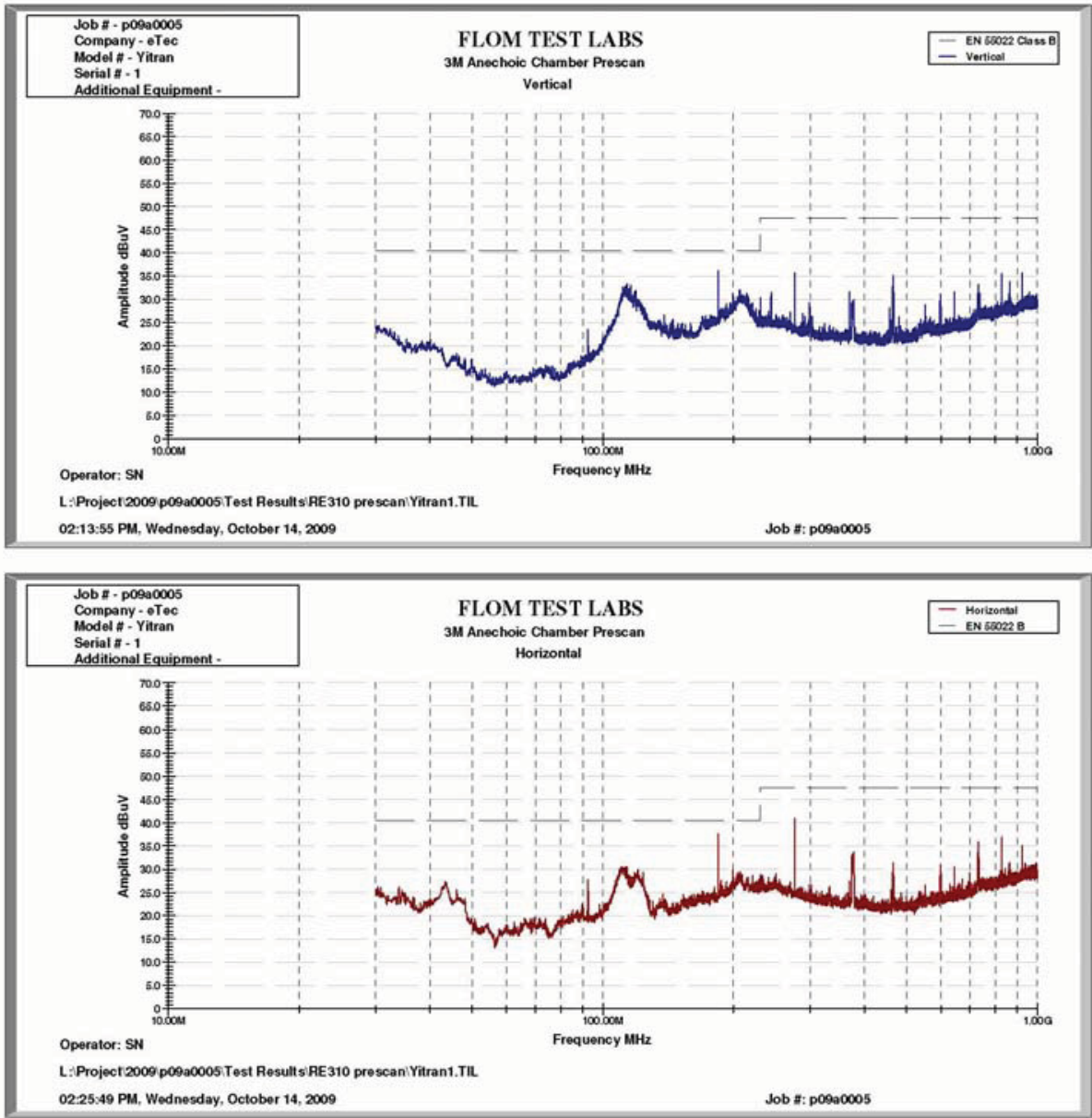

Quasi-peak Radiated Emissions

\begin{tabular}{|c|c|c|c|c|c|}
\hline $\begin{array}{c}\text { Emission Freq } \\
(\mathrm{MHz})\end{array}$ & $\begin{array}{c}\text { Measured Value } \\
(\mathrm{dBuV} / \mathrm{m})\end{array}$ & $\begin{array}{c}\text { Correction Factor } \\
(\mathrm{dB})\end{array}$ & $\begin{array}{c}\text { Corrected Value } \\
(\mathrm{dBuV} / \mathrm{m})\end{array}$ & $\begin{array}{c}\text { Limit } \\
(\mathrm{dBuV} / \mathrm{m})\end{array}$ & $\begin{array}{c}\text { Margin } \\
\mathrm{dB}\end{array}$ \\
\hline 38.57 & 18.9 & 14.5 & 33.3 & 40.0 & -6.7 \\
\hline 221.30 & 23.0 & 12.4 & 35.3 & 46.0 & -10.7 \\
\hline 368.65 & 22.1 & 17.6 & 39.7 & 46.0 & -6.3 \\
\hline 453.76 & 17.1 & 19.8 & 36.9 & 46.0 & -9.1 \\
\hline 664.47 & 8.5 & 23.5 & 32.0 & 46.0 & -14.0 \\
\hline 762.50 & 3.1 & 25.3 & 28.4 & 46.0 & -17.6 \\
\hline
\end{tabular}

Compliance Testing

3356 N. San Marcos Place, Suite 107

Chandler, Arizona 85225-7176

(866) 311-3268 phone, (480) 926-3598 fax

Page 7 of 28

p09a0005, d09a0009 Rev 6.0 
Yitran IT700 Radiated Emissions Test Setup Photos
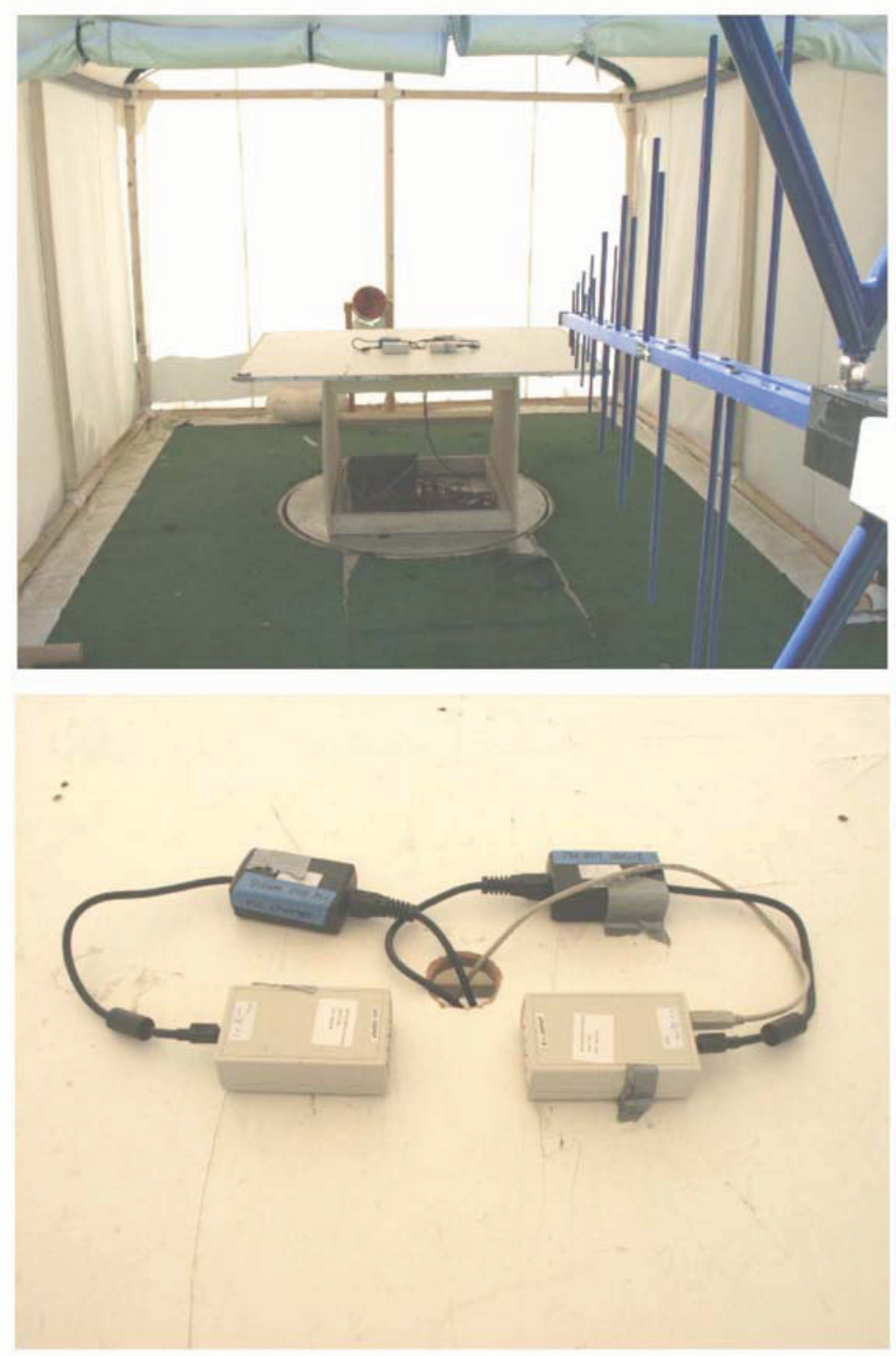

Compliance Testing

3356 N. San Marcos Place, Suite 107

Chandler, Arizona 85225-7176

(866) 311-3268 phone, (480) 926-3598 fax

Page 8 of 28 p09a0005, d09a0009 Rev 6.0 
Maxim 2990 EV Kit Peak Pre-Scans
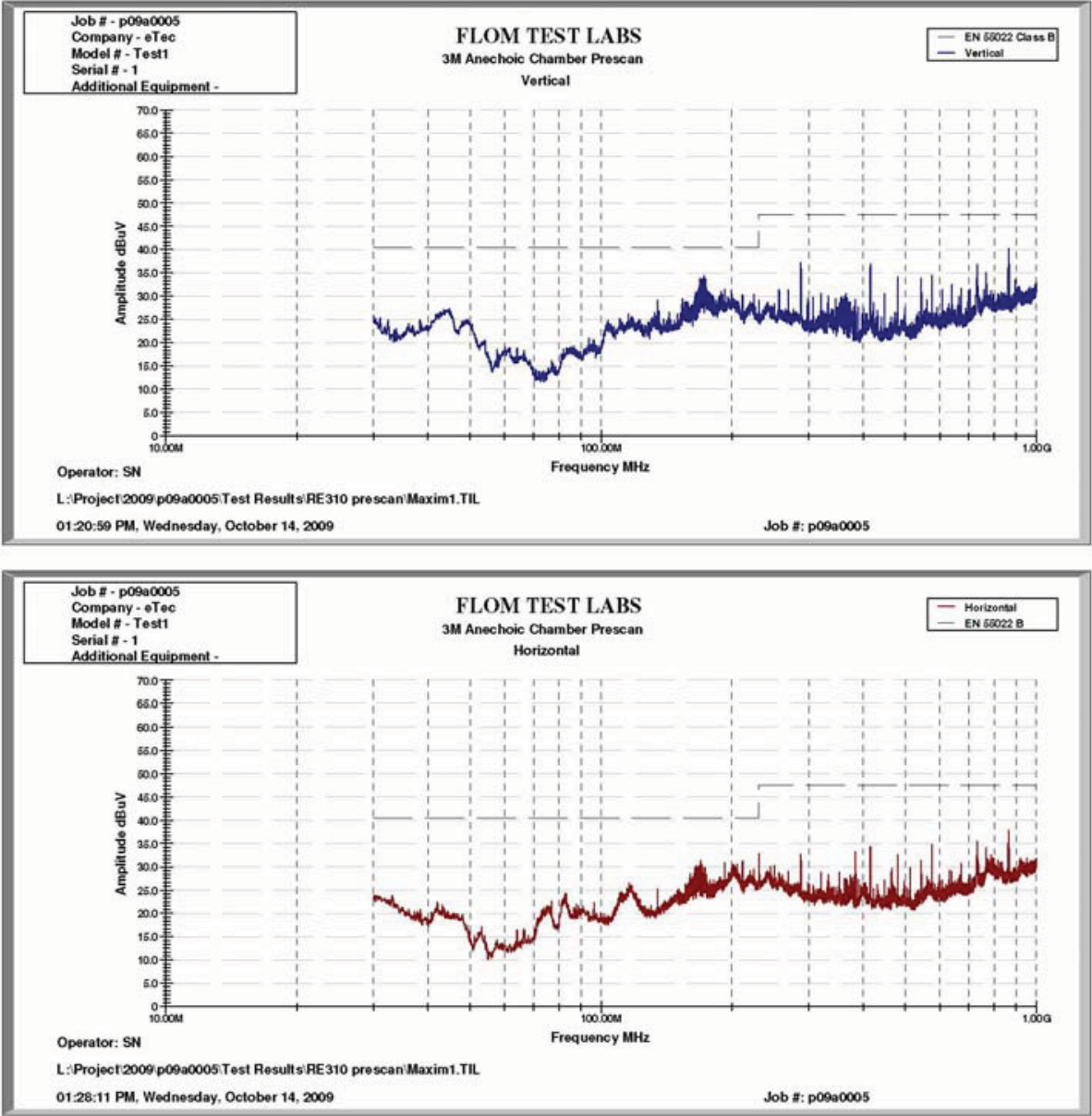

Quasi-peak Radiated Emissions-

\begin{tabular}{|c|c|c|c|c|c|}
\hline $\begin{array}{c}\text { Emission Freq } \\
(\mathrm{MHz})\end{array}$ & $\begin{array}{c}\text { Measured Value } \\
(\mathrm{dBuV} / \mathrm{m})\end{array}$ & $\begin{array}{c}\text { Correction Factor } \\
(\mathrm{dB})\end{array}$ & $\begin{array}{c}\text { Corrected Value } \\
(\mathrm{dBuV} / \mathrm{m})\end{array}$ & $\begin{array}{c}\text { Limit } \\
(\mathrm{dBuV} / \mathrm{m})\end{array}$ & $\begin{array}{c}\text { Margin } \\
\mathrm{dB}\end{array}$ \\
\hline 38.26 & 4.3 & 16.2 & 20.5 & 40.0 & -19.5 \\
\hline 61.18 & 19.9 & 6.7 & 26.6 & 40.0 & -13.4 \\
\hline 221.40 & 9.2 & 12.2 & 21.4 & 46.0 & -24.6 \\
\hline 328.60 & 18.6 & 16.3 & 34.9 & 46.0 & -11.1 \\
\hline 528.67 & 17.9 & 20.9 & 38.7 & 46.0 & -7.3 \\
\hline 896.60 & 8.0 & 27.0 & 35.0 & 46.0 & -11.0 \\
\hline
\end{tabular}

Compliance Testing

3356 N. San Marcos Place, Suite 107

Chandler, Arizona 85225-7176

(866) 311-3268 phone, (480) 926-3598 fax

Page 9 of 28

p09a0005, d09a0009 Rev 6.0 

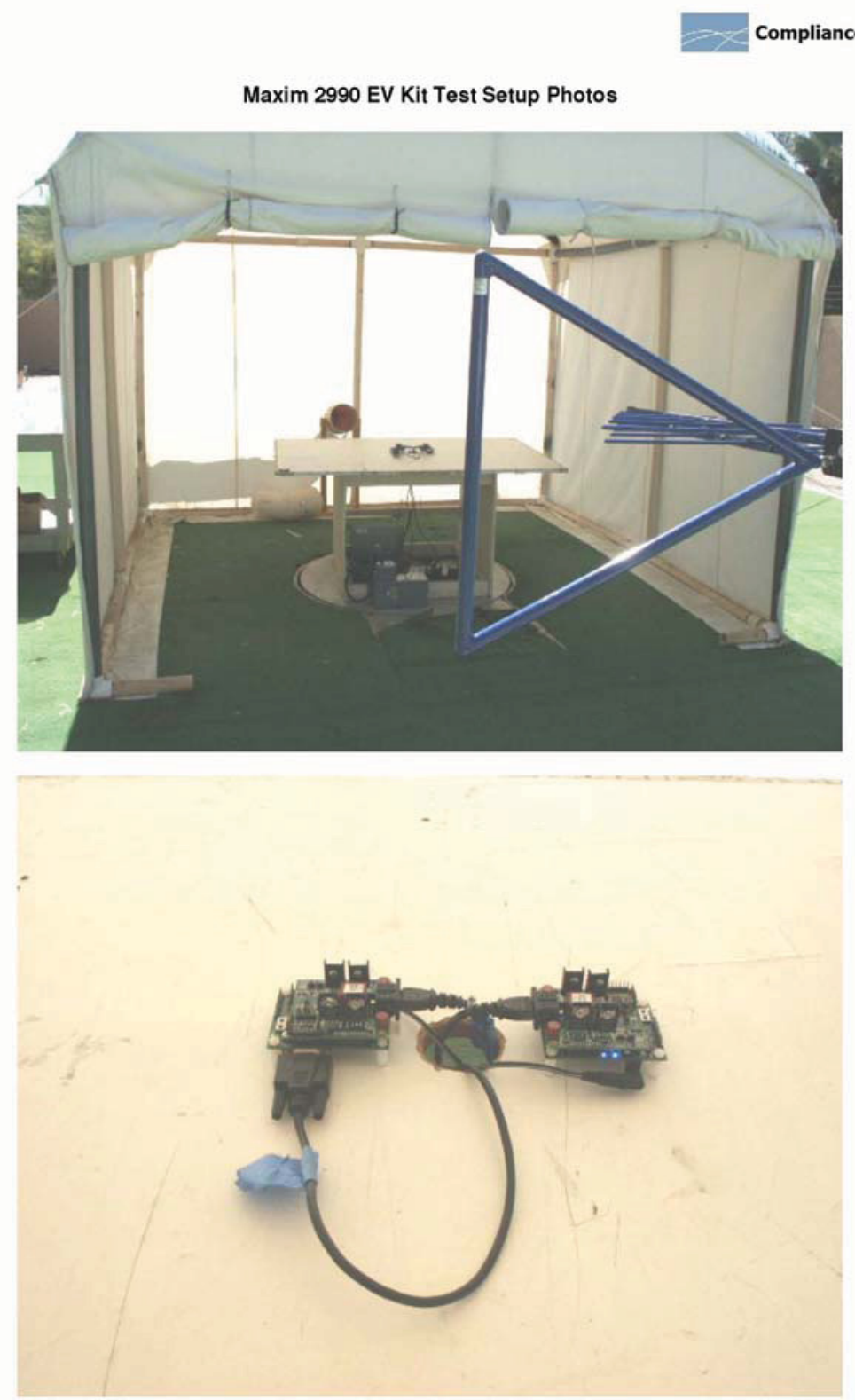

Compliance Testing

3356 N. San Marcos Place, Suite 107

Chandler, Arizona 85225-7176

(866) 311-3268 phone, (480) 926-3598 fax

Page 10 of 28 p09a0005, d09a0009 Rev 6.0 
Name of Test: Specification:

Test Equipment Utilized
Radiated Emissions
RE 310

i00033, i00267
Engineer: Sam Nickels, NCT

Test Date: October 16, 2009

Test Procedure

The EUT was tested in an Open Area Test Site (OATS) on a ground plane, set $1 \mathrm{~m}$ from the receiving antenna. An EMI Receiver was used to verify that the EUT met the requirements for Radiated Emissions. The EUT was tested by at $1 \mathrm{~m}$ height in both Vertical and Horizontal orientations. All emissions from $30 \mathrm{MHz}$ to $1 \mathrm{GHz}$ were examined.

Test Setup

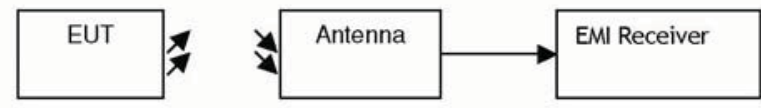

Netgear XAV101 Radiated Emissions

\begin{tabular}{|c|c|c|c|c|c|}
\hline $\begin{array}{c}\text { Emission Freq } \\
(\mathrm{MHz})\end{array}$ & $\begin{array}{c}\text { Measured Value } \\
(\mathrm{dBuV} / \mathrm{m})\end{array}$ & $\begin{array}{c}\text { Correction Factor } \\
(\mathrm{dB})\end{array}$ & $\begin{array}{c}\text { Corrected Value } \\
(\mathrm{dBuV} / \mathrm{m})\end{array}$ & $\begin{array}{c}\text { Limit } \\
(\mathrm{dBuV} / \mathrm{m})\end{array}$ & $\begin{array}{c}\text { Margin } \\
\mathrm{dB}\end{array}$ \\
\hline 40.17 & 18.7 & 13.6 & 32.3 & 48.8 & -16.5 \\
\hline 300.00 & 28.6 & 15.4 & 44.0 & 51.1 & -7.1 \\
\hline 337.50 & 26.4 & 17.0 & 43.4 & 51.9 & -8.5 \\
\hline 375.00 & 27.9 & 17.7 & 45.6 & 52.6 & -7.0 \\
\hline 500.025 & 26.9 & 20.6 & 47.5 & 53 & -5.5 \\
\hline 562.43 & 11.6 & 22.3 & 33.8 & 53 & -19.2 \\
\hline
\end{tabular}

Yitran IT700 Radiated Emissions

\begin{tabular}{|c|c|c|c|c|c|}
\hline $\begin{array}{c}\text { Emission Freq } \\
(\mathrm{MHz})\end{array}$ & $\begin{array}{c}\text { Measured Value } \\
(\mathrm{dBuV} / \mathrm{m})\end{array}$ & $\begin{array}{c}\text { Correction Factor } \\
(\mathrm{dB})\end{array}$ & $\begin{array}{c}\text { Corrected Value } \\
(\mathrm{dBuV} / \mathrm{m})\end{array}$ & $\begin{array}{c}\text { Limit A } \\
(\mathrm{dBuV} / \mathrm{m})\end{array}$ & $\begin{array}{c}\text { Margin } \\
\mathrm{dB}\end{array}$ \\
\hline 35.24 & 16.0 & 17.9 & 33.9 & 50.2 & -16.3 \\
\hline 276.50 & 22.0 & 15.3 & 37.3 & 50.6 & -13.3 \\
\hline 354.00 & 21.2 & 17.6 & 38.9 & 52.2 & -13.3 \\
\hline 375.00 & 19.8 & 18.0 & 37.8 & 52.6 & -14.8 \\
\hline 842.60 & 13.1 & 26.5 & 39.6 & 53 & -13.4 \\
\hline 925.68 & 6.8 & 27.9 & 34.7 & 53 & -18.3 \\
\hline
\end{tabular}

Maxim 2990 EV Kit Radiated Emissions

\begin{tabular}{|c|c|c|c|c|c|}
\hline $\begin{array}{c}\text { Emission Freq } \\
(\mathrm{MHz})\end{array}$ & $\begin{array}{c}\text { Measured Value } \\
(\mathrm{dBuV} / \mathrm{m})\end{array}$ & $\begin{array}{c}\text { Correction Factor } \\
(\mathrm{dB})\end{array}$ & $\begin{array}{c}\text { Corrected Value } \\
(\mathrm{dBuV} / \mathrm{m})\end{array}$ & $\begin{array}{c}\text { Limit A } \\
(\mathrm{dBuV} / \mathrm{m})\end{array}$ & $\begin{array}{c}\text { Margin } \\
\mathrm{dB}\end{array}$ \\
\hline 38.26 & 14.6 & 14.6 & 29.2 & 49.3 & -20.1 \\
\hline 64.18 & 21.8 & 6.7 & 28.6 & 43.7 & -15.1 \\
\hline 328.60 & 23.4 & 16.3 & 39.7 & 51.7 & -12.0 \\
\hline 528.67 & 19.0 & 20.9 & 39.8 & 53 & -13.2 \\
\hline 869.60 & 5.3 & 27.0 & 32.3 & 53 & -20.7 \\
\hline 968.00 & 9.3 & 28.6 & 37.9 & 53 & -15.1 \\
\hline
\end{tabular}

Compliance Testing

3356 N. San Marcos Place, Suite 107

Chandler, Arizona 85225-7176

(866) 311-3268 phone, (480) 926-3598 fax

Page 11 of 28

p09a0005, d09a0009 Rev 6.0 


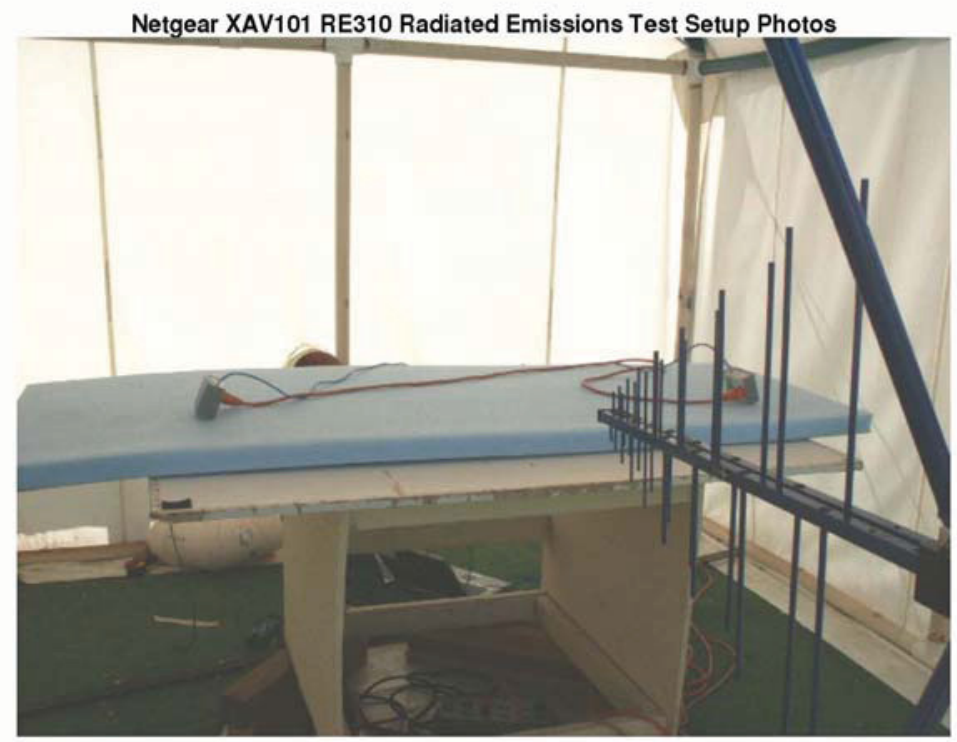

Yitran IT700 RE310 Radiated Emissions Photos Test Setup Continued

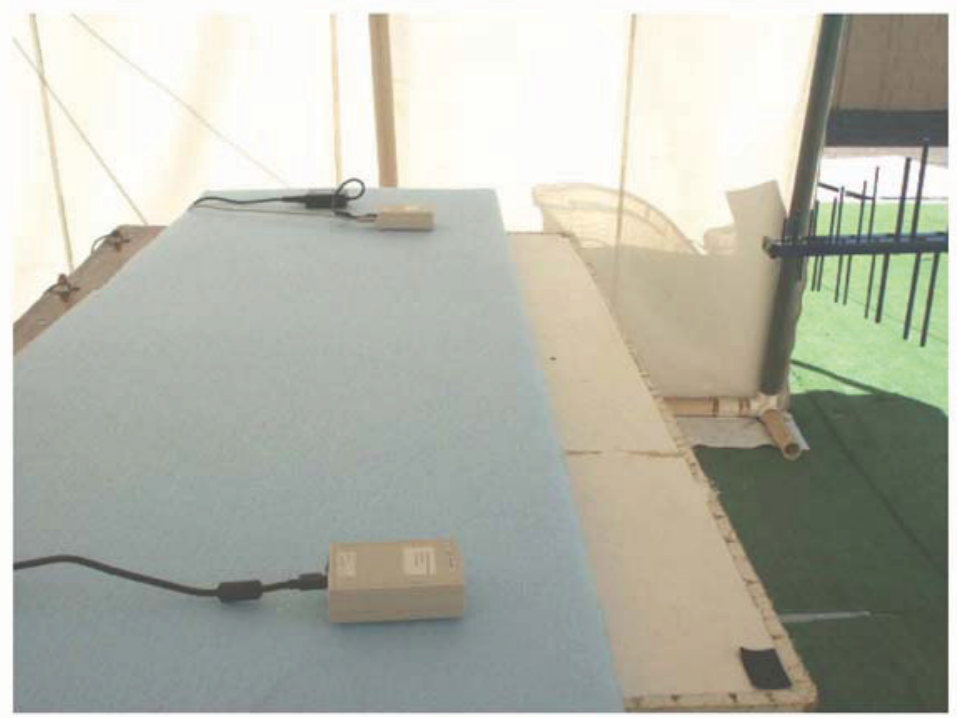

Compliance Testing

3356 N. San Marcos Place, Suite 107

Chandler, Arizona 85225-7176

(866) 311-3268 phone, (480) 926-3598 fax

Page 12 of 28 p09a0005, d09a0009 Rev 6.0 
Maxim 2990 EV Kit RE310 Radiated Emissions Test Setup Photos

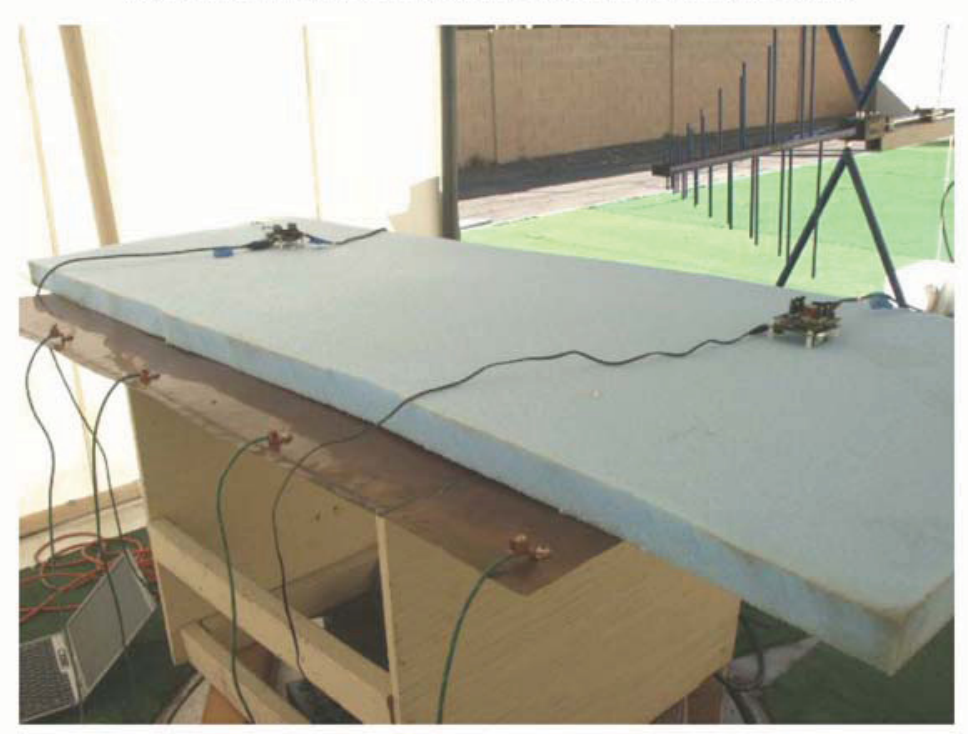

Compliance Testing

3356 N. San Marcos Place, Suite 107

Chandler, Arizona 85225-7176

(866) 311-3268 phone, (480) 926-3598 fax

Page 13 of 28 p09a0005, d09a0009 Rev 6.0 
Name of Test: FCC Specification: Ford-AC:

Test Equipment Utilized
FCC Power line Conducted Emissions, Ford-AC Conducted Emissions

15.107

CE420; EU1 \& G1 ranges passed

i00033, i00270
Engineer: Sam Nickels, NCT

Test Date: October 19, 2009

\section{Test Procedure}

The EUT power cable connected to a LISN and the monitored output of the LISN was connected directly to an EMI Receiver. The conducted emissions from $150 \mathrm{kHz}$ to $30 \mathrm{MHz}$ were monitored and compared to the specification limits.

Test Setup

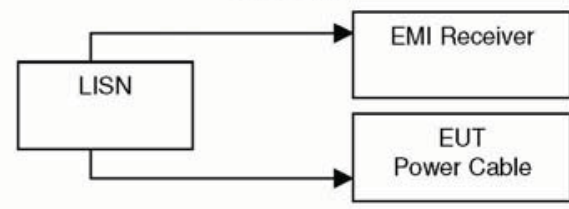

Netgear XAV101 Conducted Emissions Line 1 Neutral Peak Graph

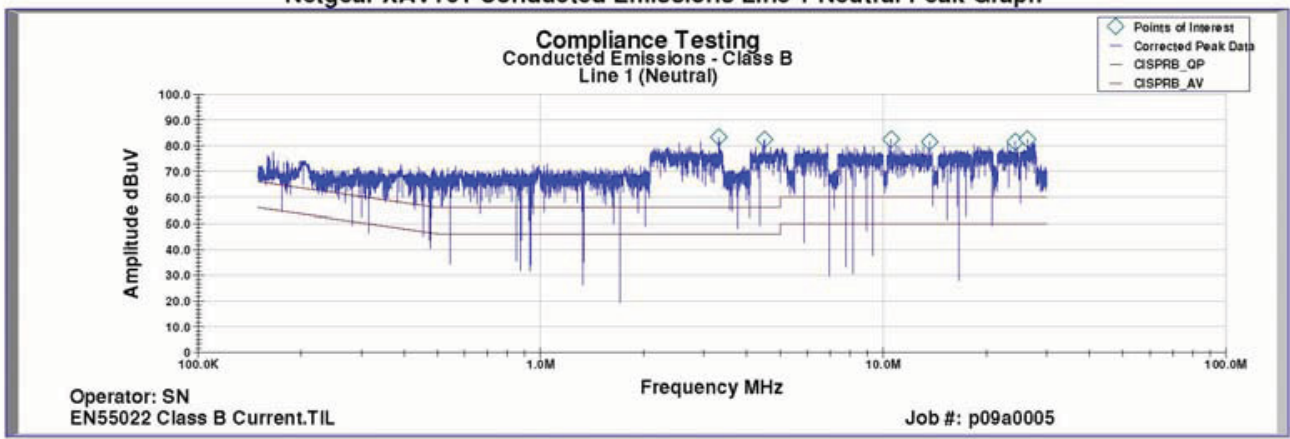

Netgear XAV101 Conducted Emissions Line 2 Neutral Peak Graph

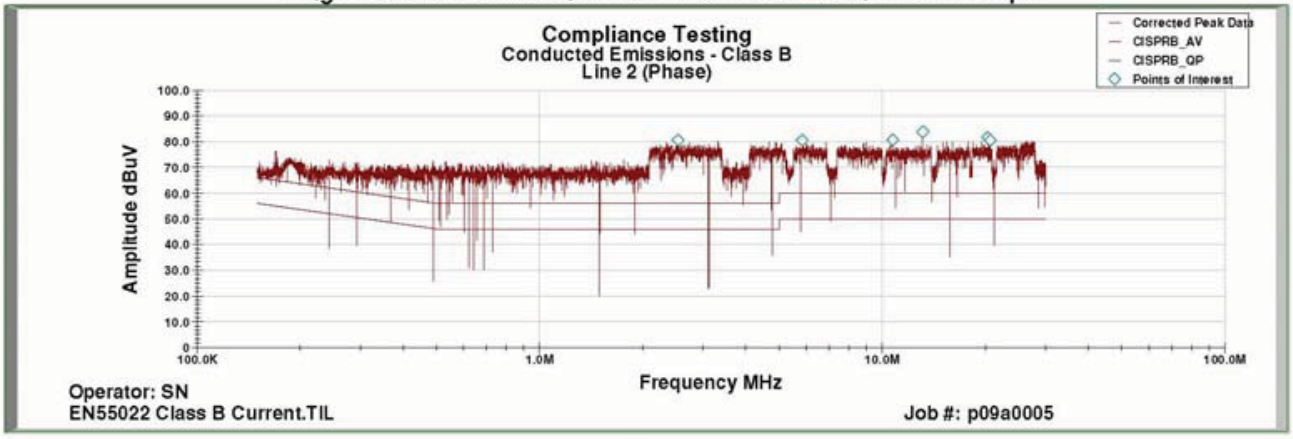

Compliance Testing

3356 N. San Marcos Place, Suite 107

Chandler, Arizona 85225-7176

(866) 311-3268 phone, (480) 926-3598 fax

Page 14 of 28

p09a0005, d09a0009 Rev 6.0 
Netgear XAV101 Line 1 Neutral AVG Detector

\begin{tabular}{|c|c|c|c|c|c|c|c|}
\hline Frequency $(\mathrm{MHz})$ & $\begin{array}{c}\text { Uncorrected } \\
\text { Data } \\
(\mathrm{dBuV})\end{array}$ & $\begin{array}{c}\text { LISN Corr } \\
\text { Fact (dB) }\end{array}$ & $\begin{array}{c}\text { Cable } \\
\text { Loss }(\mathrm{dB})\end{array}$ & $\begin{array}{c}\text { Attenuator } \\
(\mathrm{dB})\end{array}$ & $\begin{array}{c}\text { Final L1 } \\
(\mathrm{dBuV})\end{array}$ & $\begin{array}{c}\text { Limit } \\
(\mathrm{dBuV})\end{array}$ & $\begin{array}{c}\text { AVG } \\
\text { Margin } \\
(\mathrm{dB})\end{array}$ \\
\hline $26.36 \mathrm{MHz}$ & 62.72 & 0.20 & 1.03 & 10.00 & 73.95 & 50.00 & 23.95 \\
\hline $24.21 \mathrm{MHz}$ & 61.64 & 0.20 & 0.99 & 10.00 & 72.83 & 50.00 & 22.83 \\
\hline $13.67 \mathrm{MHz}$ & 60.49 & 0.00 & 0.70 & 10.00 & 71.19 & 50.00 & 21.19 \\
\hline $10.55 \mathrm{MHz}$ & 60.97 & 0.00 & 0.58 & 10.00 & 71.55 & 50.00 & 21.55 \\
\hline $4.49 \mathrm{MHz}$ & 62.05 & 0.00 & 0.41 & 10.00 & 72.45 & 46.00 & 26.45 \\
\hline $3.32 \mathrm{MHz}$ & 61.28 & 0.00 & 0.33 & 10.00 & 71.62 & 46.00 & 25.62 \\
\hline
\end{tabular}

\begin{tabular}{|c|c|c|c|c|c|c|c|}
\hline \multicolumn{1}{|c|}{ Netgear XAV101 Line 2 Phase AVG Detector } \\
\hline Frequency (MHz) & $\begin{array}{c}\text { Uncorrected } \\
\text { Data } \\
(\mathrm{dBuV})\end{array}$ & $\begin{array}{c}\text { LISN Corr } \\
\text { Fact (dB) }\end{array}$ & $\begin{array}{c}\text { Cable } \\
\text { Loss (dB) }\end{array}$ & $\begin{array}{c}\text { Attenuator } \\
(\mathrm{dB})\end{array}$ & $\begin{array}{c}\text { Final L1 } \\
(\mathrm{dBuV})\end{array}$ & $\begin{array}{c}\text { Limit } \\
(\mathrm{dBuV})\end{array}$ & $\begin{array}{c}\text { AVG } \\
\text { Margin } \\
(\mathrm{dB})\end{array}$ \\
\hline $20.70 \mathrm{MHz}$ & 63.03 & 0.10 & 0.92 & 10.00 & 74.05 & 50.00 & 24.05 \\
\hline $20.31 \mathrm{MHz}$ & 63.55 & 0.10 & 0.91 & 10.00 & 74.56 & 50.00 & 24.56 \\
\hline $13.08 \mathrm{MHz}$ & 62.91 & 0.00 & 0.67 & 10.00 & 73.59 & 50.00 & 23.59 \\
\hline $10.74 \mathrm{MHz}$ & 63.20 & 0.00 & 0.59 & 10.00 & 73.79 & 50.00 & 23.79 \\
\hline $5.85 \mathrm{MHz}$ & 62.81 & 0.00 & 0.47 & 10.00 & 73.28 & 50.00 & 23.28 \\
\hline $2.53 \mathrm{MHz}$ & 62.21 & 0.00 & 0.29 & 10.00 & 72.50 & 46.00 & 26.50 \\
\hline
\end{tabular}

\begin{tabular}{|c|c|c|c|c|c|c|c|}
\hline \multicolumn{1}{|c|}{ Netgear XAV101 Line 1 Neutral QP Detector } \\
\hline Frequency (MHz) & $\begin{array}{c}\text { Uncorrected } \\
\text { Data } \\
(\mathrm{dBuV})\end{array}$ & $\begin{array}{c}\text { LISN Corr } \\
\text { Fact (dB) }\end{array}$ & $\begin{array}{c}\text { Cable } \\
\text { Loss (dB) }\end{array}$ & $\begin{array}{c}\text { Attenuator } \\
(\mathrm{dB})\end{array}$ & $\begin{array}{c}\text { Final } \\
\text { L1 } \\
(\mathrm{dBuV})\end{array}$ & $\begin{array}{c}\text { Limit } \\
(\mathrm{dBuV})\end{array}$ & $\begin{array}{c}\text { QP } \\
\text { Margin } \\
(\mathrm{dB})\end{array}$ \\
\hline $26.36 \mathrm{MHz}$ & 75.11 & 0.20 & 1.03 & 10.00 & 86.34 & 60.00 & 26.34 \\
\hline $24.21 \mathrm{MHz}$ & 73.77 & 0.20 & 0.99 & 10.00 & 84.96 & 60.00 & 24.96 \\
\hline $13.67 \mathrm{MHz}$ & 73.23 & 0.00 & 0.70 & 10.00 & 83.93 & 60.00 & 23.93 \\
\hline $10.55 \mathrm{MHz}$ & 74.19 & 0.00 & 0.58 & 10.00 & 84.77 & 60.00 & 24.77 \\
\hline $4.49 \mathrm{MHz}$ & 73.97 & 0.00 & 0.41 & 10.00 & 84.38 & 56.00 & 28.38 \\
\hline $3.32 \mathrm{MHz}$ & 73.38 & 0.00 & 0.33 & 10.00 & 83.71 & 56.00 & 27.71 \\
\hline
\end{tabular}

Netgear XAV101 Line 2 Phase QP Detector

\begin{tabular}{|c|c|c|c|c|c|c|c|}
\hline Frequency $(\mathrm{MHz})$ & $\begin{array}{c}\text { Uncorrected } \\
\text { Data } \\
(\mathrm{dBuV})\end{array}$ & $\begin{array}{c}\text { LISN Corr } \\
\text { Fact }(\mathrm{dB})\end{array}$ & $\begin{array}{c}\text { Cable } \\
\text { Loss }(\mathrm{dB})\end{array}$ & $\begin{array}{c}\text { Attenuator } \\
(\mathrm{dB})\end{array}$ & $\begin{array}{c}\text { Final } \\
\text { L1 } \\
(\mathrm{dBuV})\end{array}$ & $\begin{array}{c}\text { Limit } \\
(\mathrm{dBuV})\end{array}$ & $\begin{array}{c}\text { QP } \\
\text { Margin } \\
(\mathrm{dB})\end{array}$ \\
\hline $20.70 \mathrm{MHz}$ & 73.75 & 0.10 & 0.92 & 10.00 & 84.77 & 60.00 & 24.77 \\
\hline $20.31 \mathrm{MHz}$ & 75.48 & 0.10 & 0.91 & 10.00 & 86.49 & 60.00 & 26.49 \\
\hline $13.08 \mathrm{MHz}$ & 74.18 & 0.00 & 0.67 & 10.00 & 84.85 & 60.00 & 24.85 \\
\hline $10.74 \mathrm{MHz}$ & 74.28 & 0.00 & 0.59 & 10.00 & 84.87 & 60.00 & 24.87 \\
\hline $5.85 \mathrm{MHz}$ & 73.59 & 0.00 & 0.47 & 10.00 & 84.06 & 60.00 & 24.06 \\
\hline $2.53 \mathrm{MHz}$ & 72.68 & 0.00 & 0.29 & 10.00 & 82.97 & 56.00 & 26.97 \\
\hline
\end{tabular}

Compliance Testing

3356 N. San Marcos Place, Suite 107

Chandler, Arizona 85225-7176

(866) 311-3268 phone, (480) 926-3598 fax

Page 15 of 28 p09a0005, d09a0009 Rev 6.0 
Netgear XAV101 Conducted Emissions Test Setup Photos:
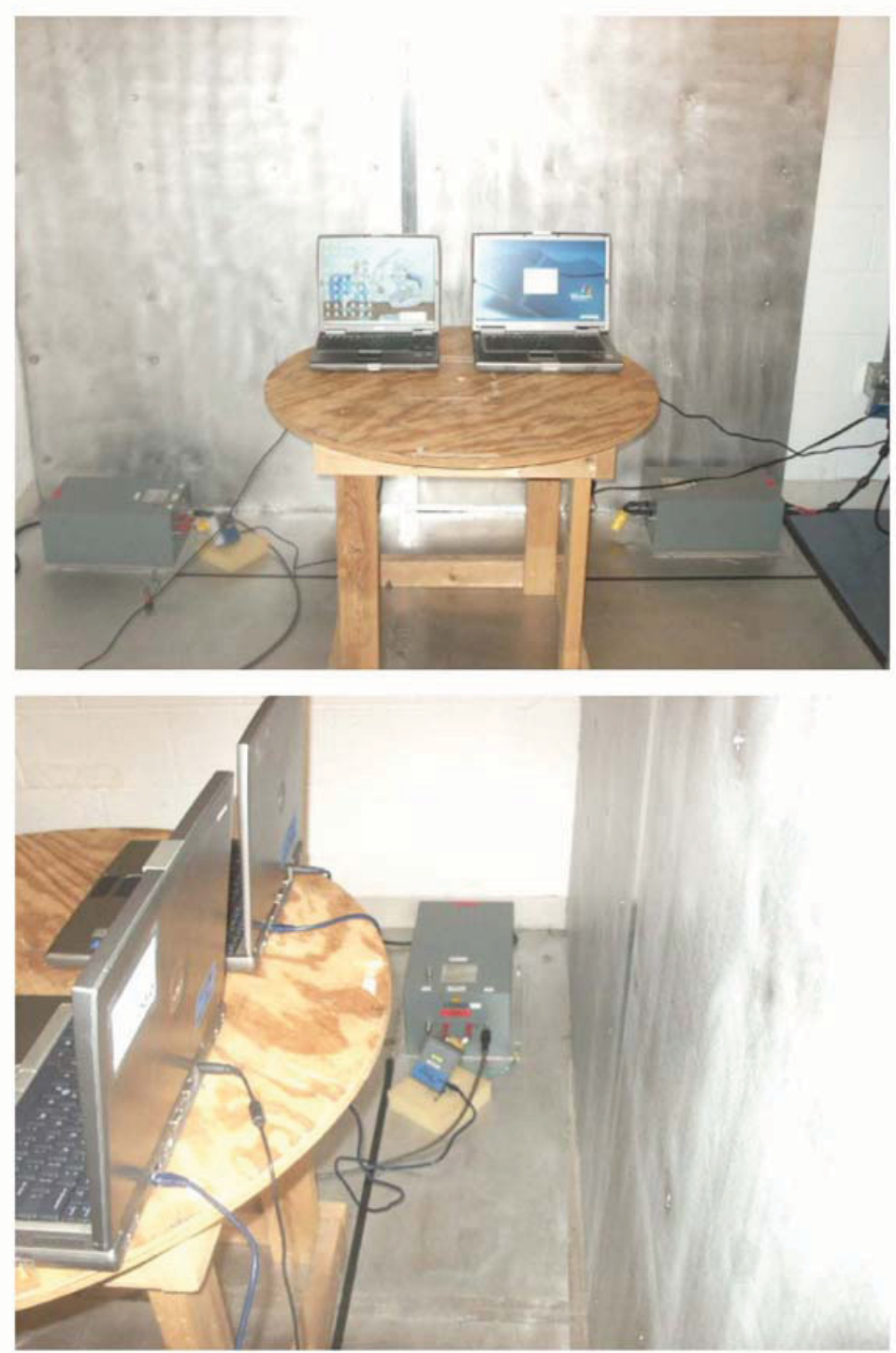

Compliance Testing

3356 N. San Marcos Place, Suite 107

Chandler, Arizona 85225-7176

(866) 311-3268 phone, (480) 926-3598 fax

Page 16 of 28 p09a0005, d09a0009 Rev 6.0 
Compliance Testing

Yitran IT700 Conducted Emissions Line 1 Neutral Peak Graph

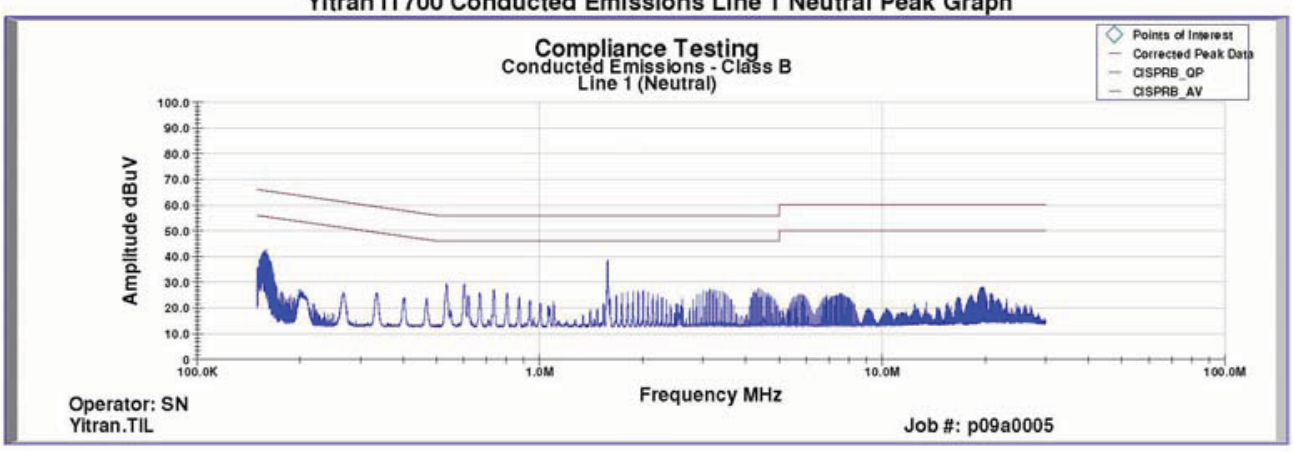

Yitran IT700 Conducted Emissions Line 2 Neutral Peak Graph

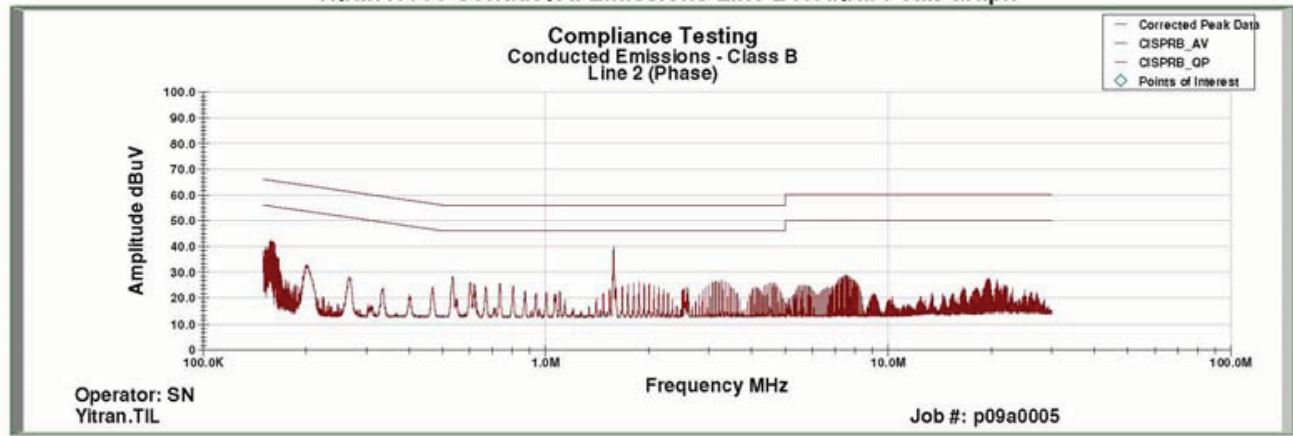

All peak emissions were below the quasi-peak and average limits.

Compliance Testing

3356 N. San Marcos Place, Suite 107

Chandler, Arizona 85225-7176

(866) 311-3268 phone, (480) 926-3598 fax

Page 17 of 28

p09a0005, d09a0009 Rev 6.0 


\section{Yitran IT700 Conducted Emissions Photos Continued:}
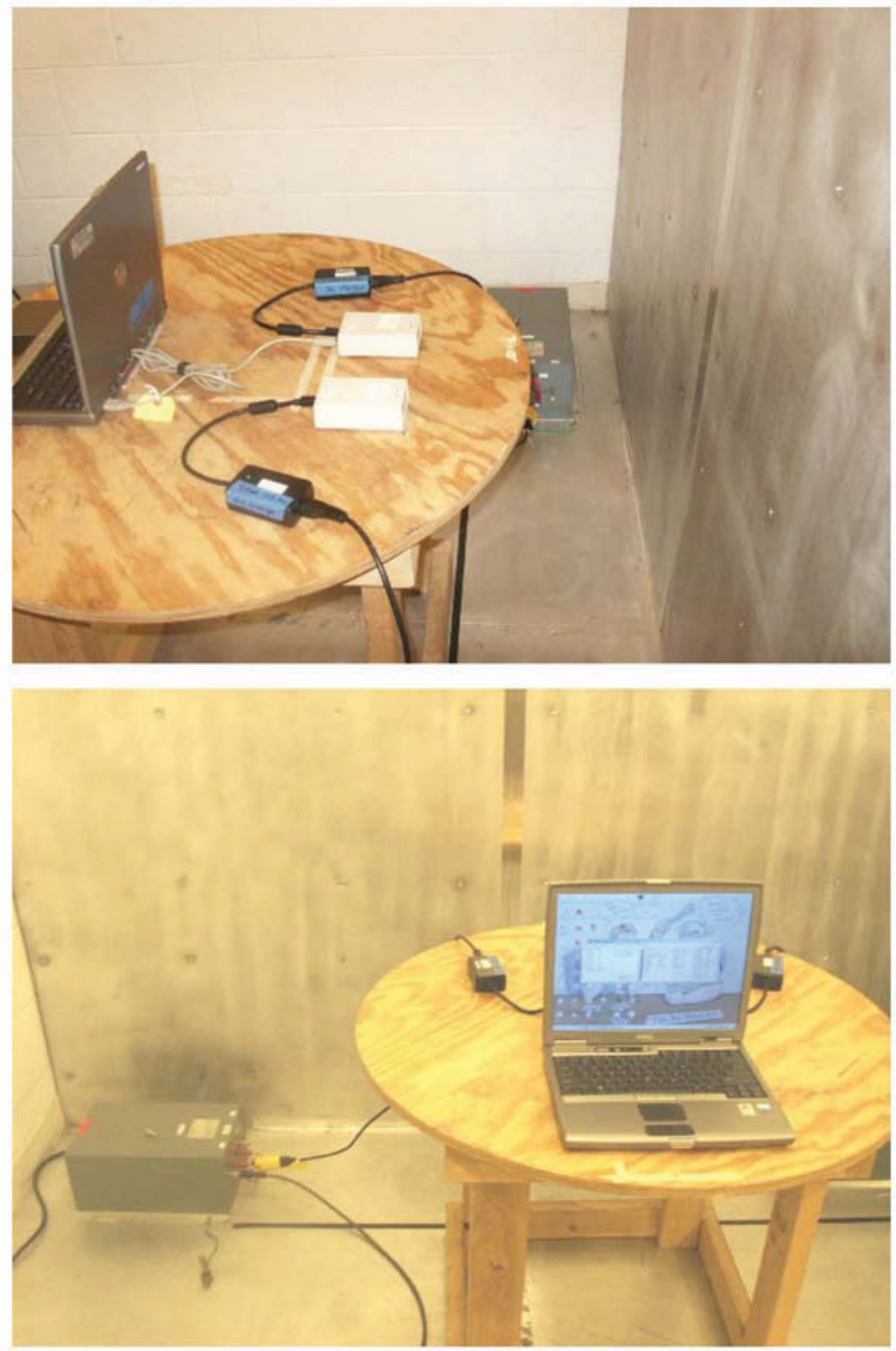

Compliance Testing

3356 N. San Marcos Place, Suite 107

Chandler, Arizona 85225-7176

(866) 311-3268 phone, (480) 926-3598 fax

Page 18 of 28

p09a0005, d09a0009 Rev 6.0 
Compliance Testing

Maxim 2990 EV Kit Conducted Emissions Line 1 Neutral Peak Graph

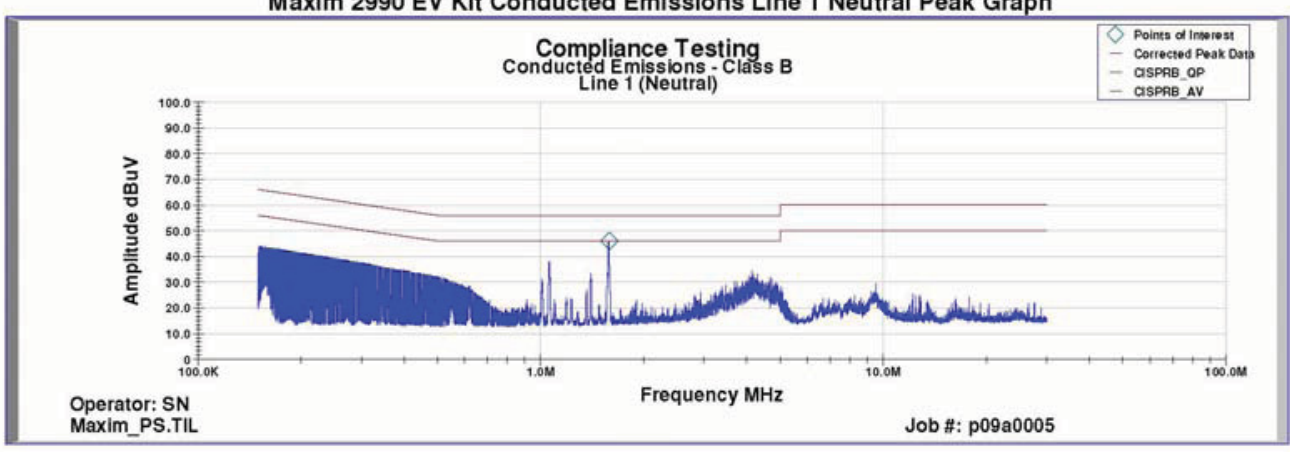

Maxim 2990 EV Kit Conducted Emissions Line 2 Neutral Peak Graph

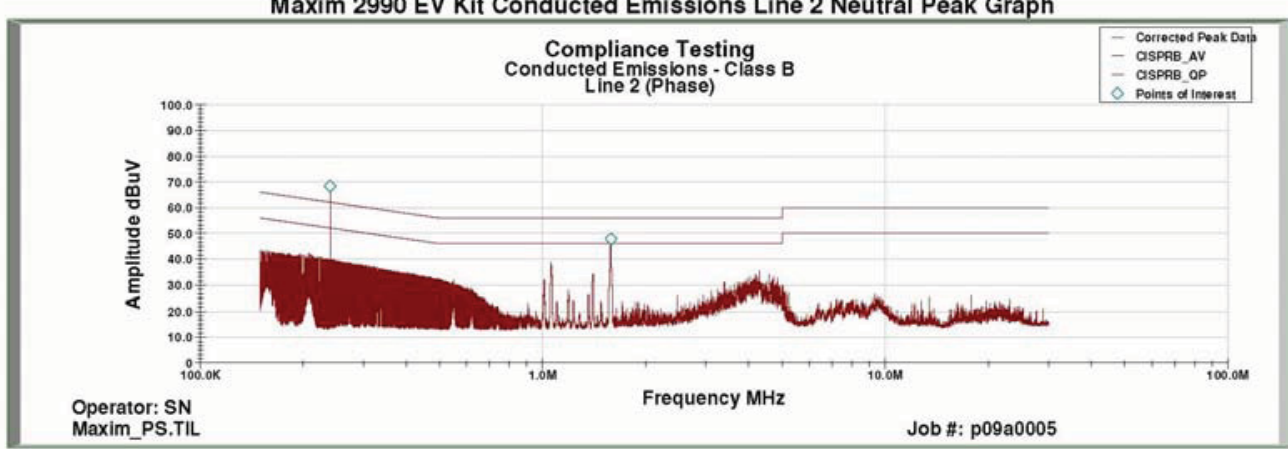

Compliance Testing

3356 N. San Marcos Place, Suite 107

Chandler, Arizona 85225-7176

(866) 311-3268 phone, (480) 926-3598 fax

Page 19 of 28

p09a0005, d09a0009 Rev 6.0 
Maxim 2990 EV Kit Line 1 Neutral AVG Detector

\begin{tabular}{|c|c|c|c|c|c|c|c|}
\hline Frequency $(\mathrm{MHz})$ & $\begin{array}{c}\text { Uncorrected } \\
\text { Data } \\
(\mathrm{dBuV})\end{array}$ & $\begin{array}{c}\text { LISN Corr } \\
\text { Fact }(\mathrm{dB})\end{array}$ & $\begin{array}{c}\text { Cable } \\
\text { Loss } \\
(\mathrm{dB})\end{array}$ & $\begin{array}{c}\text { Attenuator } \\
(\mathrm{dB})\end{array}$ & $\begin{array}{c}\text { Final } \\
\text { L1 } \\
(\mathrm{dBuV})\end{array}$ & $\begin{array}{c}\text { Limit } \\
(\mathrm{dBuV})\end{array}$ & $\begin{array}{c}\text { AVG } \\
\text { Margin } \\
(\mathrm{dB})\end{array}$ \\
\hline $1.58 \mathrm{MHz}$ & 32.01 & 0.00 & 0.23 & 10.00 & 42.24 & 46.00 & -3.76 \\
\hline
\end{tabular}

Maxim 2990 EV Kit Line 2 Phase AVG Detector

\begin{tabular}{|c|c|c|c|c|c|c|c|}
\hline Frequency $(\mathrm{MHz})$ & $\begin{array}{c}\text { Uncorrected } \\
\text { Data } \\
(\mathrm{dBuV})\end{array}$ & $\begin{array}{c}\text { LISN Corr } \\
\text { Fact }(\mathrm{dB})\end{array}$ & $\begin{array}{c}\text { Cable } \\
\text { Loss } \\
(\mathrm{dB})\end{array}$ & $\begin{array}{c}\text { Attenuator } \\
(\mathrm{dB})\end{array}$ & $\begin{array}{c}\text { Final } \\
\text { L1 } \\
(\mathrm{dBuV})\end{array}$ & $\begin{array}{c}\text { Limit } \\
(\mathrm{dBuV})\end{array}$ & $\begin{array}{c}\text { AVG } \\
\text { Margin } \\
(\mathrm{dB})\end{array}$ \\
\hline $1.58 \mathrm{MHz}$ & 33.98 & 0.00 & 0.23 & 10.00 & 44.21 & 46.00 & -1.79 \\
\hline $227.34 \mathrm{KHz}$ & -3.31 & 0.19 & 0.07 & 10.00 & 6.94 & 53.79 & -46.85 \\
\hline
\end{tabular}

Maxim 2990 EV Kit TX Line 1 Neutral QP Detector

\begin{tabular}{|c|c|c|c|c|c|c|c|}
\hline Frequency $(\mathrm{MHz})$ & $\begin{array}{c}\text { Uncorrected } \\
\text { Data } \\
(\mathrm{dBuV})\end{array}$ & $\begin{array}{c}\text { LISN Corr } \\
\text { Fact }(\mathrm{dB})\end{array}$ & $\begin{array}{c}\text { Cable } \\
\text { Loss } \\
(\mathrm{dB})\end{array}$ & $\begin{array}{c}\text { Attenuator } \\
(\mathrm{dB})\end{array}$ & $\begin{array}{c}\text { Final } \\
\text { L1 } \\
(\mathrm{dBuV})\end{array}$ & $\begin{array}{c}\text { Limit } \\
(\mathrm{dBuV})\end{array}$ & $\begin{array}{c}\text { QP } \\
\text { Margin } \\
(\mathrm{dB})\end{array}$ \\
\hline $1.58 \mathrm{MHz}$ & 35.15 & 0.00 & 0.23 & 10.00 & 45.38 & 56.00 & -10.62 \\
\hline
\end{tabular}

Maxim 2990 EV Kit Line 2 Phase QP Detector

\begin{tabular}{|c|c|c|c|c|c|c|c|}
\hline Frequency $(\mathrm{MHz})$ & $\begin{array}{c}\text { Uncorrected } \\
\text { Data } \\
(\mathrm{dBuV})\end{array}$ & $\begin{array}{c}\text { LISN Corr } \\
\text { Fact }(\mathrm{dB})\end{array}$ & $\begin{array}{c}\text { Cable } \\
\text { Loss } \\
(\mathrm{dB})\end{array}$ & $\begin{array}{c}\text { Attenuator } \\
(\mathrm{dB})\end{array}$ & $\begin{array}{c}\text { Final } \\
\text { L1 } \\
(\mathrm{dBuV})\end{array}$ & $\begin{array}{c}\text { Limit } \\
(\mathrm{dBuV})\end{array}$ & $\begin{array}{c}\text { QP } \\
\text { Margin } \\
(\mathrm{dB})\end{array}$ \\
\hline $1.5807 \mathrm{MHz}$ & 37.47 & 0.00 & 0.23 & 10.00 & 47.70 & 56.00 & -8.30 \\
\hline $227.34 \mathrm{KHz}$ & 24.31 & 0.19 & 0.07 & 10.00 & 34.56 & 63.79 & -29.23 \\
\hline
\end{tabular}

All other emissions were greater that $30 \mathrm{~dB}$ below the quasi peak or average limit.

Compliance Testing

3356 N. San Marcos Place, Suite 107

Chandler, Arizona 85225-7176

Page 20 of 28

(866) 311-3268 phone, (480) 926-3598 fax

p09a0005, d09a0009 Rev 6.0 
Maxim 2990 EV Kit Conducted Emissions Photos Continued:
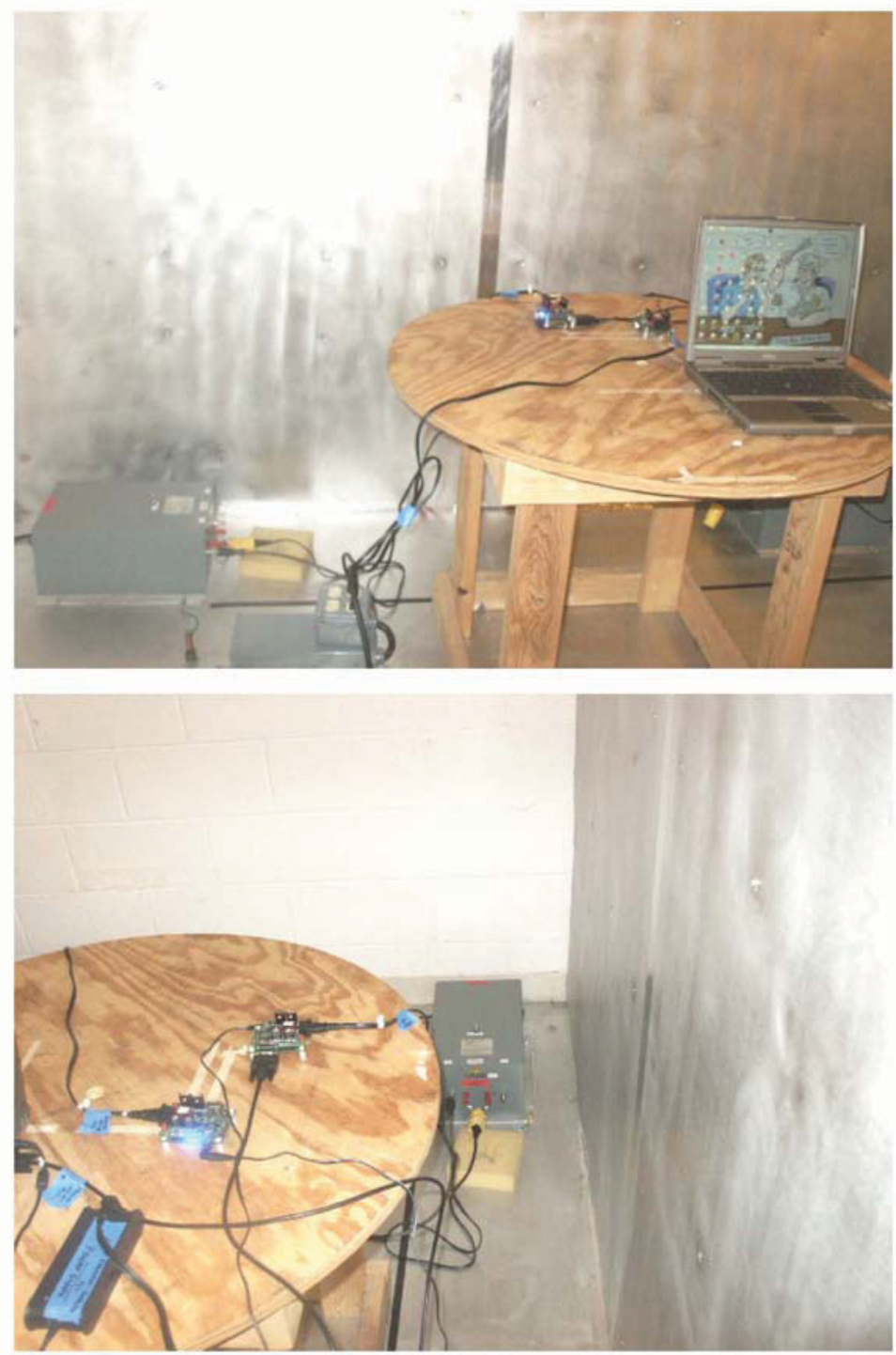

Compliance Testing

3356 N. San Marcos Place, Suite 107

Chandler, Arizona 85225-7176

(866) 311-3268 phone, (480) 926-3598 fax

Page 21 of 28 p09a0005, d09a0009 Rev 6.0 
Name of Test:

Specification:

Test Equipment Utilized
Radiated Immunity- Bulk Current Injection (BCI)

RI112

Engineer: Sam Nickels, NCT

100031, i00262, i00263, i00275, Test Date: October 20, 2009

i00358, i00315, i00041

\section{Test Procedure}

The EUT was tested using a signal generator and amplifier to inject an RF signal into the $\mathrm{BCl}$ Injection probe-this was placed at strategic locations on the EUT wiring/power lines. This was tested to 'Level 1' as required by the customer in accordance with Ford- $\mathrm{AC} \mathrm{BCl}$ (engineering development testing). The $\mathrm{BCl}$ probe was placed on the USB or Serial connector to the laptop, the power lines, and both ends of the power supply/transformers.

Results:

- The only anomalous behavior observed was $90 \mathrm{MHz}-100 \mathrm{MHz}$ on the Yitran IT700 test units only. There was a "hardware reset" indicated in the software, but communications did not stop. The error went away after passing $100 \mathrm{MHz}$ (CW \& AM). According to the Ford-AC Specification, this was Function Performance Status // behavior (being that the anomaly/test unit recovered without intervention after the RF was removed)

- The Netgear XAV101 and Maxim 2990 EV Kit exhibited no anomalous behavior (Function Performance Status I).

Compliance Testing

3356 N. San Marcos Place, Suite 107

Chandler, Arizona 85225-7176

(866) 311-3268 phone, (480) 926-3598 fax

Page 22 of 28

p09a0005, d09a0009 Rev 6.0 
Netgear XAV101 Rl112 Radiated Immunity Test Setup Photos
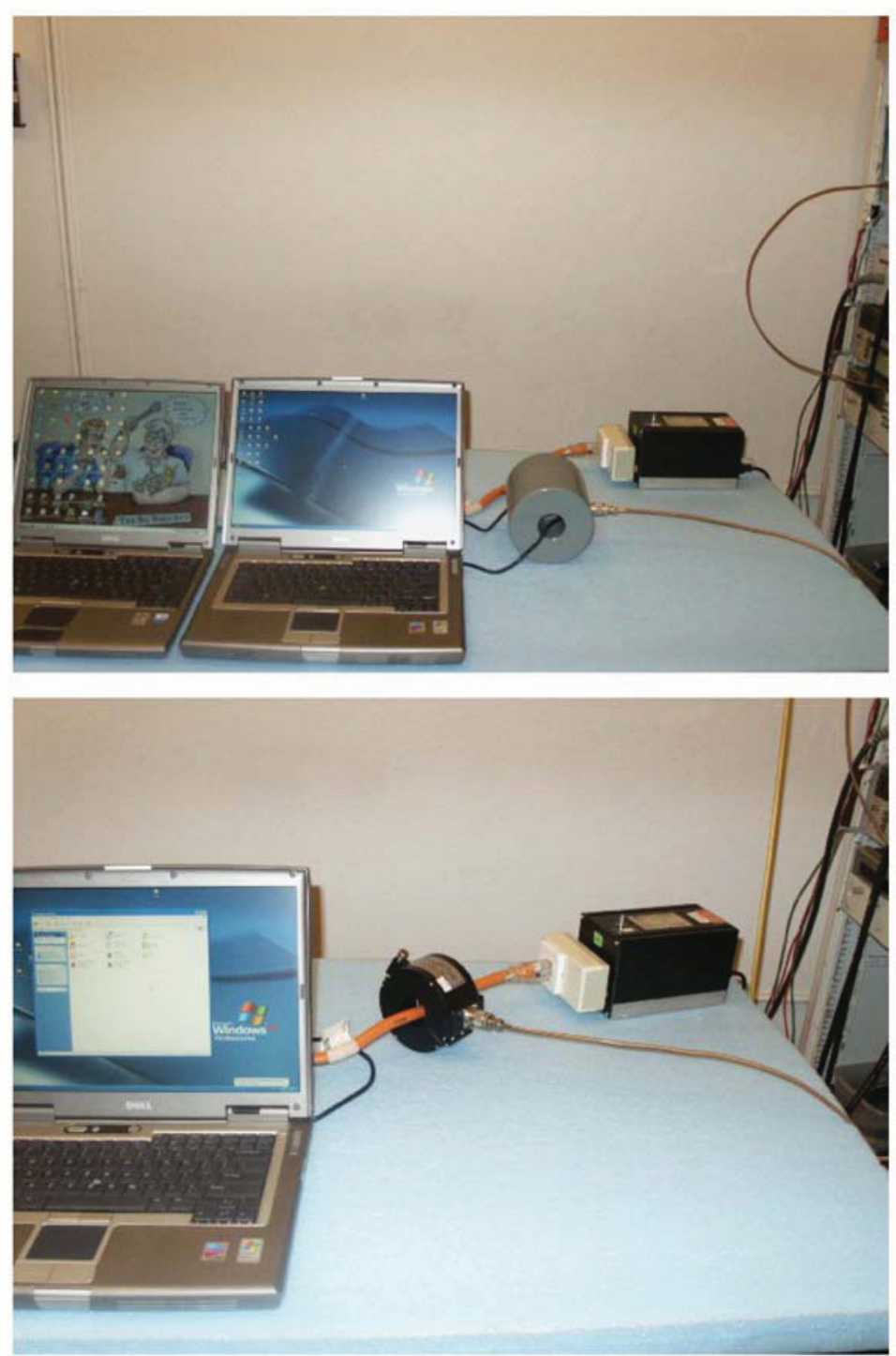

Compliance Testing

3356 N. San Marcos Place, Suite 107

Chandler, Arizona 85225-7176

(866) 311-3268 phone, (480) 926-3598 fax

Page 23 of 28 p09a0005, d09a0009 Rev 6.0 

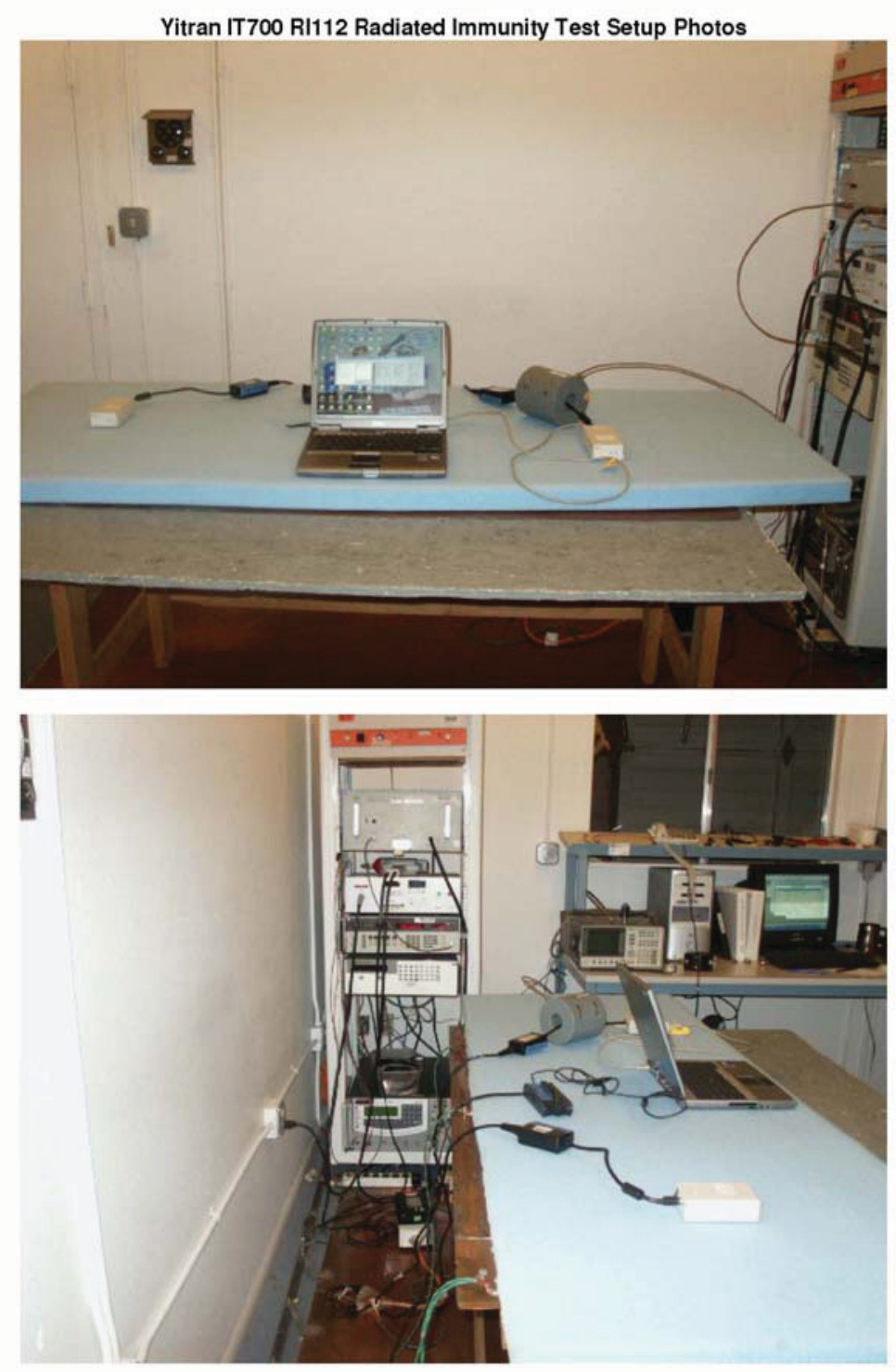

Compliance Testing

3356 N. San Marcos Place, Suite 107

Chandler, Arizona 85225-7176

(866) 311-3268 phone, (480) 926-3598 fax

Page 24 of 28 p09a0005, d09a0009 Rev 6.0 
Maxim 2990 EV Kit RI112 Radiated Immunity Test Setup Photos Continued:
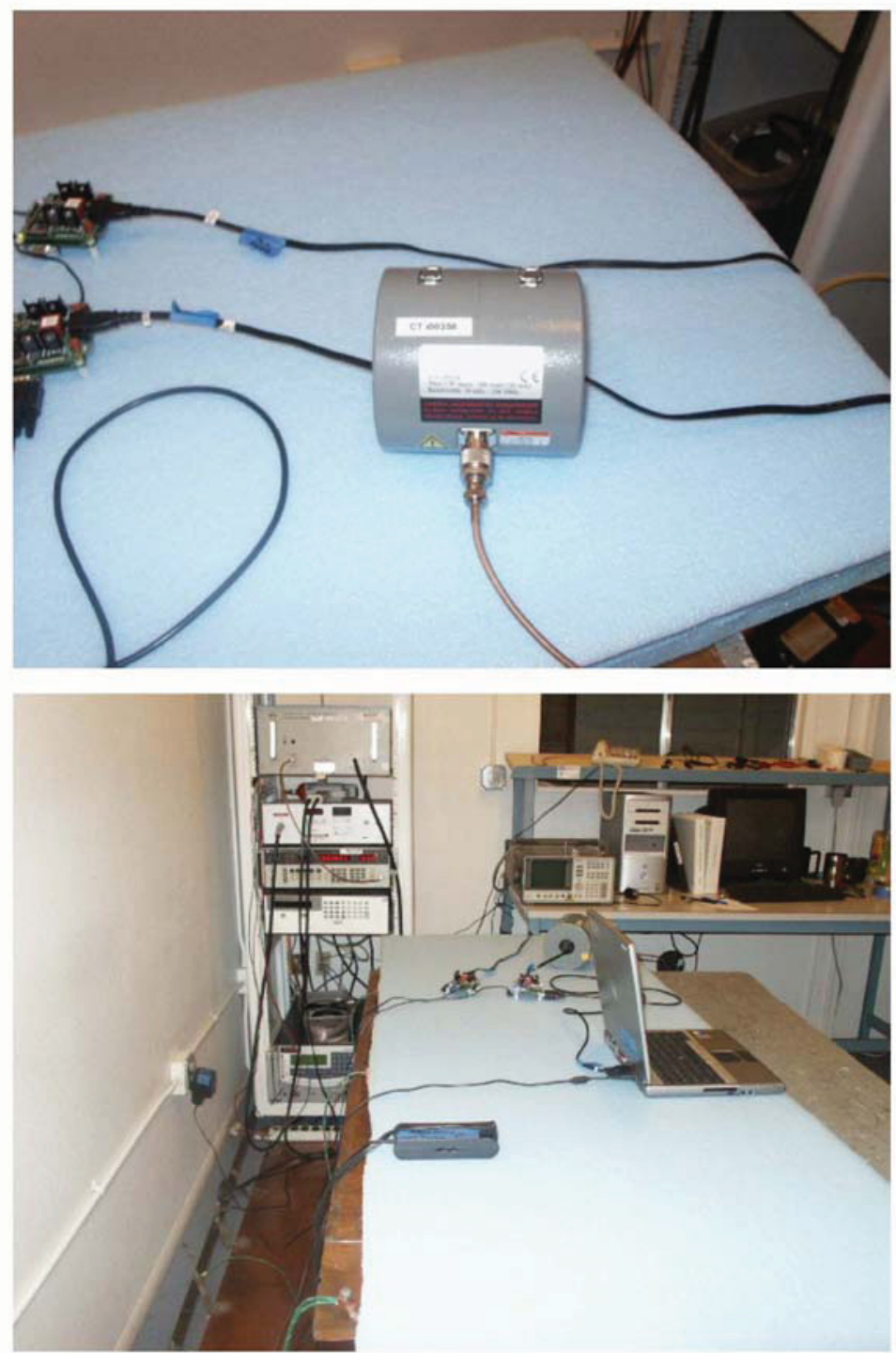

Compliance Testing

3356 N. San Marcos Place, Suite 107

Chandler, Arizona 85225-7176

(866) 311-3268 phone, (480) 926-3598 fax

Page 25 of 28 p09a0005, d09a0009 Rev 6.0 
Name of Test:

Specification:

Test Equipment Utilized
Radiated Immunity- ALSE Method
Rl114

100266, i00280, i00281, i00310
Engineer: Sam Nickels, NCT Test Date: October 13, 2009

\section{Test Procedure}

The EUT was tested using a signal generator and RF amplifier to inject an RF signal into an antenna located in an anechoic chamber. This was tested to 'Level 1' as required by the customer in accordance with the Ford-AC ALSE (Absorber Lined Shielded Enclosure) method.

\section{Results:}

- None of the test samples exhibited anomalous behavior (Function Performance Status I).

Netgear XAV101 RI114 Radiated Immunity (ALSE) Setup Photos:

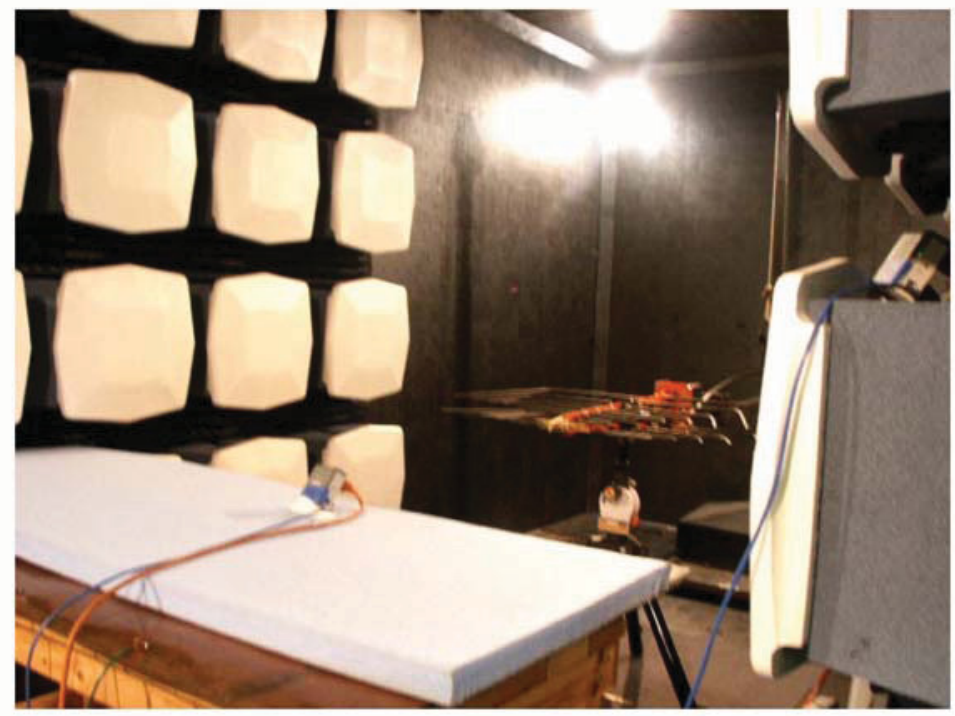

Compliance Testing

3356 N. San Marcos Place, Suite 107

Chandler, Arizona 85225-7176

(866) 311-3268 phone, (480) 926-3598 fax 
Yitran IT700 RI114 Radiated Immunity (ALSE) Setup Photos Continued:
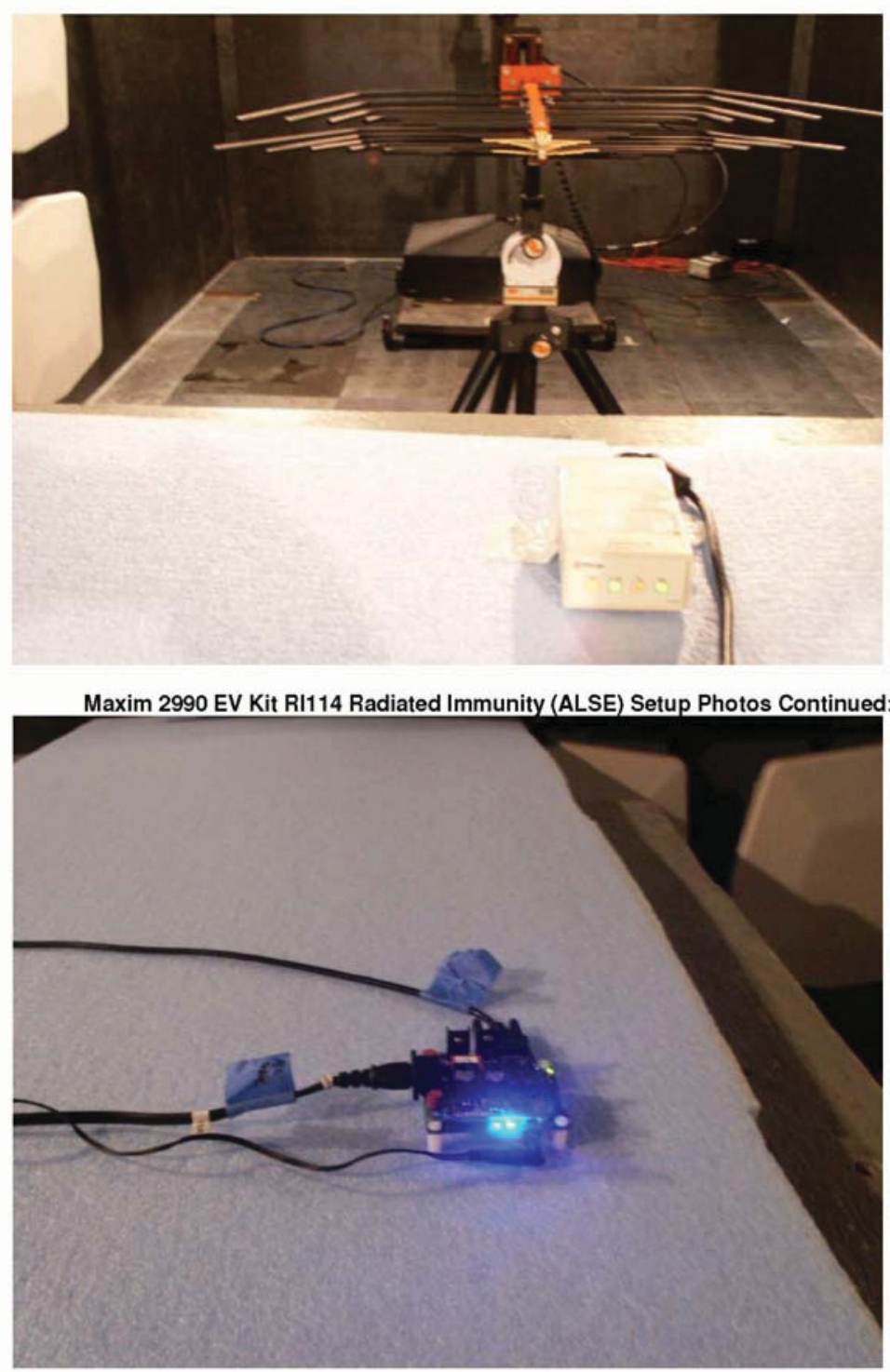

Compliance Testing

3356 N. San Marcos Place, Suite 107

Chandler, Arizona 85225-7176

(866) 311-3268 phone, (480) 926-3598 fax

Page 27 of 28 p09a0005, d09a0009 Rev 6.0 
Test Equipment Utilized

\begin{tabular}{|l|l|l|l|l|l|}
\hline $\begin{array}{c}\text { Asset } \\
\#\end{array}$ & \multicolumn{1}{|c|}{ Manufacturer } & Model & \multicolumn{1}{c|}{$\begin{array}{c}\text { Serial } \\
\text { Number }\end{array}$} & Cal Cycle & Calibration Due \\
\hline i00031 & HP & $8656 \mathrm{~A}$ & $2402 \mathrm{~A} 06180$ & When used & Verified \\
\hline i00033 & HP & $8546 \mathrm{~A}$ & $3325 \mathrm{~A} 00122$ & $12 \mathrm{mo}$. & $10 / 14 / 2009^{*}$ \\
\hline 100041 & Amplifier Research & $50 \mathrm{~W} 1000 \mathrm{~A}$ & 17772 & When use & Verified \\
\hline i00262 & 200 W 3dB Attenuator & $50 \mathrm{FH}-003-200$ & 160851 & When used & Verified \\
\hline i00263 & 200 W 3dB Attenuator & $50 \mathrm{FH}-003-200$ & 146304 & When used & Verified \\
\hline i00266 & Rohde\&Schwarz & SMT03 & $82611 / 005$ & When used & Verified \\
\hline i00267 & Schaffner & CBL611C & 2910 & 24 mo. & $11 / 6 / 2009$ \\
\hline i00270 & FCC & FCC-LISN-50-50-2-01 & 2050 & 24 mo. & $9 / 17 / 2010$ \\
\hline i00275 & EIN & 440LA & 231 & When used & Verified \\
\hline i00280 & Amplifier Research & AT5080 & 312715 & When used & Verified \\
\hline i00281 & Amplifier Research & 60 S1G3 & 300262 & When used & Verified \\
\hline i00310 & EMPower & 2024 BBS1C4ALP & 1009 D/C0609 & When used & Verified \\
\hline 100315 & Solar Electric Co. & Type 9142-1N & 063802 & 12 mo. & $9 / 17 / 2010$ \\
\hline i00358 & Fischer Custom Comm. & F-120-9A & 09104 & 12 mo. & $1 / 23 / 2010$ \\
\hline
\end{tabular}

In addition to the above listed equipment standard RF connectors and cables were utilized in the testing of the described equipment. Prior to testing these components were tested to verify proper operation.

* This item was granted a 30-day calibration extension per our calibration program under ISO17025.

END OF TEST REPORT

Compliance Testing

3356 N. San Marcos Place, Suite 107

Chandler, Arizona 85225-7176

(866) 311-3268 phone, (480) 926-3598 fax

Page 28 of 28

p09a0005, d09a0009 Rev 6.0 


\title{
Appendix B-9 \\ Industrial Application Test Results (Maxim 2990/2991)
}

\section{eTec Test Results}

\author{
Kedar Ponkshe \\ 08/31/2009-09/01/2009
}

\section{Car with onboard Charger}

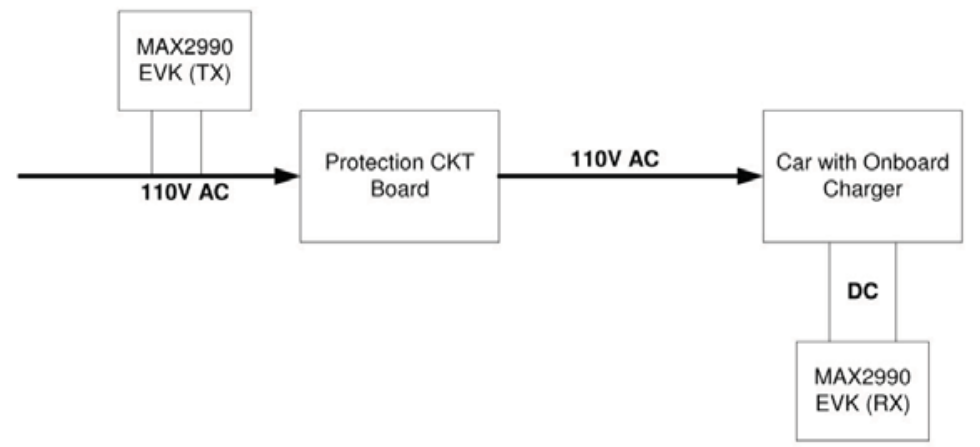

Test Setup 


\section{Car with onboard Charger Signal Capture}

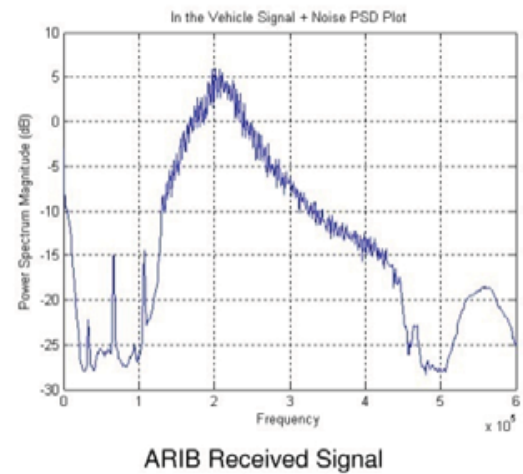

- The communication frequency band is $130 \mathrm{KHz}-450 \mathrm{KHz}$

- There is about $15 \mathrm{~dB}$ more attenuation on high frequencies due to the Protection CKT.

- The frequency band of $250 \mathrm{~K}-450 \mathrm{~K}$ might give better performance.

- Data rate $=21 \mathrm{Kbps}$ in ARIB ROBO mode and $\sim 85 \mathrm{Kbps}$ in Normal Mode

- Cenelec $=4.5 \mathrm{Kbps}$ in ROBO mode and $19 \mathrm{Kbps}$ in Normal mode.

Maxim Confidential

\section{A Charger}

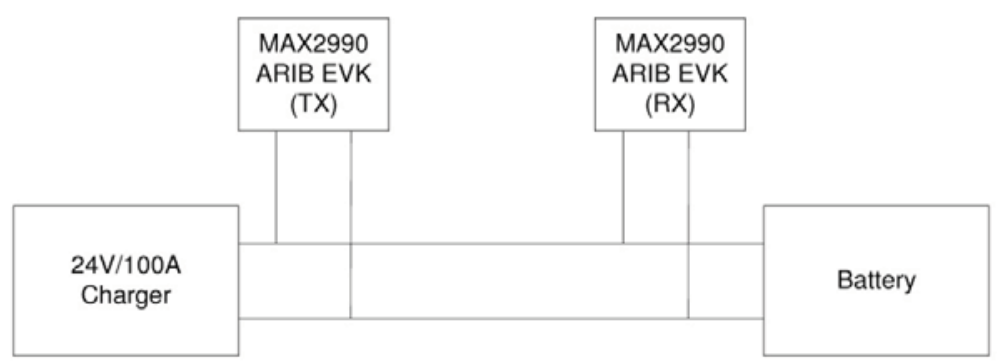

Test Setup 


\section{A Charger Signal Capture}

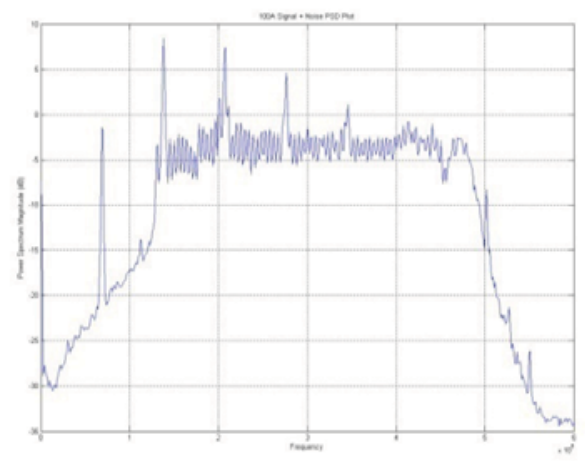

Received Signal

- Switching frequency harmonics @ $140 \mathrm{KHz}, 210 \mathrm{KHz}, 280 \mathrm{KHz} \ldots$

- It will be better to use high frequency band between $250 \mathrm{KHz}-450 \mathrm{KHz}$ to get better performance.

- ARIB Data rate $=21 \mathrm{Kbps}$ in ROBO mode and $85 \mathrm{Kbps}$ in Normal Mode

Maxim Confidential

\section{A Charger}

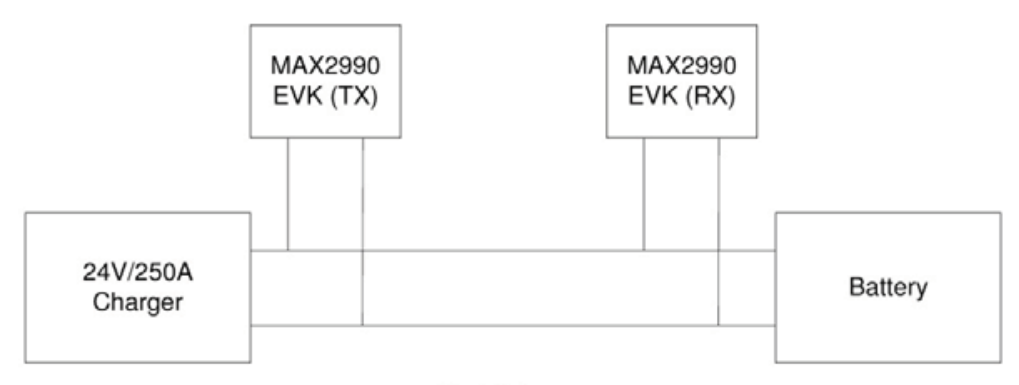

Test Setup 


\section{A Charger Signal Capture}

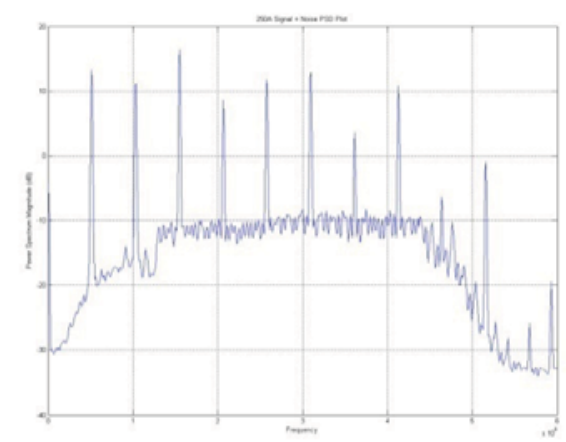

Received Signal

- $69 \mathrm{KHz}$ Switching frequency harmonics in the communication band.

- The harmonics are $20 \mathrm{~dB}$ stronger than the OFDM signal.

- Cenelec or ARIB not functional in ROBO mode

- $300 \mathrm{KHz}-450 \mathrm{KHz}$ special frequency band to reduce the no. of harmonics in communication band.

- Data rate $=11 \mathrm{Kbps}$ in $\mathrm{ROBO}$ mode with $5 \%$ retransmission.

\section{Conclusion/Comments}

- ARIB band gives the best performance for tests with onboard car charger and 100A DC charger. The data rate $\sim 85 \mathrm{Kbps}$ in Normal mode and $\sim 21 \mathrm{Kbps}$ in ROBO mode. However from the signal captures it might be better to use a frequency band between $250 \mathrm{KHz}-450 \mathrm{KHz}$.

- 250A DC charger has harmonics $\sim 20 \mathrm{~dB}$ stronger than OFDM signal.

- The communication on 250A DC charger test is possible when the frequency band is modified to $300 \mathrm{KHz}-450 \mathrm{KHz}$. The data rate is $11 \mathrm{Kbps}$ in ROBO mode.

- The PLC communication data rate is dependent on the amount of switching harmonics created by the charger unit as seen in the 250A DC charger test. But the ROBO mode on MAX2990 was able recover the data in this noisy condition.

- The start and end frequencies for communication frequency band are programmable in MAX2990. So for different powerline conditions, the same chip can be programmed to communicate in user defined communication frequency band. 
Appendix C

Cost Tables 


\section{Appendix C-1 \\ Development Costs}

\section{DEVELOPMENT COSTS FOR INTEGRATION}

\section{Chipset Evaluation Chip manufacturer}

The cost of implementing an engineering model using a select chip set under high part counts using the range of $10 \mathrm{~K}$ piece orders assume chip

manufacturer support for the following items

1: Boot Sector Code, free emulator, and program kit

2: Power Supply Design Support

3: Free Support for configuration of the Chip Set

\section{Chipset Evaluation Design and Development Costs}

Note:

Assume the Design Engineer is familiar with PLC technology and

with the writing software for the chipset.

1: Identification of Signal and Power features of the chipset

$\$ 1,000.00$

2: Determine Power and Signal support for the chipset based on what is available from existing eTec chargers

3: Assume a close match exists between items 1 \& 2

Design an engineering model in support of the chipset.

The engineering model is a high level signal and power flow graph.

4: Develop Source Code using the development tools Offered by the manufacturer.

\{THIS ASSUMES THE CHIP MANUFACTURER

PROVIDES ALL THE NEEDED LIBRARIES\}

5: Design the Power Supply section

$\$ 2000,00$

6: Layout and design the PCB board

$\$ 2,500.00$

7: Test \& Debug the system

$\$ 4,000.00$

8: FCC Testing

$\$ 30,000$

Total Cost Estimate for Development

$\$ 46,500.00$ 


\section{Appendix C-2 \\ Part Costs}

\begin{tabular}{|c|c|c|c|c|}
\hline \multicolumn{5}{|c|}{ BILL OF MATERIAL ESTIMATE F } \\
\hline \multicolumn{5}{|c|}{ Per unit cost at 10000 order level } \\
\hline QNTY & Item Description & Digikey Part Number & Part Cost & Total Cost \\
\hline 1 & MAXIM 2990 & IN PRODUCTION & 8.50 & 8.50 \\
\hline 2 & MAXIM 2991 & IN DEVELOPMENT & 4.5 & 9 \\
\hline 1 & $\operatorname{MAX} 1589$ & N/A & & 0 \\
\hline 1 & $\operatorname{MAX} 3232$ & N/A & & 0 \\
\hline 1 & ZENER DIODE SMT & S3M-FDITR-ND & 0.116 & 0.116 \\
\hline 1 & CRYSTAL $8.0 \mathrm{MHZ}$ & 887-1077-2-ND & 0.225 & 0.225 \\
\hline 1 & CRYSTAL 960 THOUGH HOLE & ECS-761.21-S-130T-ND & 0.54 & 0.54 \\
\hline 2 & POLARIZED CAPS & 08052C102J4T2A-PD & 0.056 & 0.112 \\
\hline 17 & SMT CAPS 805 & 08052C102J4T2A-ND & 0.056 & 0.952 \\
\hline 18 & SMT CAPS 402 & 399-1023-2-ND & 0.067 & 1.206 \\
\hline 14 & SMT RESITORS 402 & RHM2.OKJTR-ND & 0.00373 & 0.05222 \\
\hline 16 & SMT RESITORS 805 & RHM.004AJTR-ND & 0.419 & 6.704 \\
\hline 1 & POWER TRANSORMER & 14A-20-512-ND & 12.5876 & 12.5876 \\
\hline 6 & JUNCTION FIELD EFFECT TRANSISTOR & 568-1965-2-ND & 0.21663 & 1.29978 \\
\hline 4 & ZENER DIODES 805 & MAZ8360GMLTR-ND & 0.08072 & 0.32288 \\
\hline 4 & TVS DIODES & 3.0SMCJ2OADITR-ND & 0.3528 & 1.4112 \\
\hline 90 & TOTAL NUMBER OF PARTS & TOTAL COST OF MATERIAL & & 43.03 \\
\hline $\begin{array}{c}\text { NOTE: } \\
1\end{array}$ & \multicolumn{4}{|c|}{$\begin{array}{l}\text { PRICES COVERING PASSIVE COMPONENTS SUCH AS CAPACITORS, RESISTORS, } \\
\text { INDUCTORS, WERE SELECTED FROM THE MOST COMMONLY ORDERED. }\end{array}$} \\
\hline 2 & \multicolumn{4}{|c|}{$\begin{array}{l}\text { PRICES INVOLVING ALL OTHER MATERIALS ARE BASED ON WHAT IS AVAILABLE } \\
\text { AS IT AFFECTS THE DESIGN }\end{array}$} \\
\hline 3 & \multicolumn{4}{|c|}{$\begin{array}{l}\text { PART ATTRIBUTES AS THEY APPLY TO PASSIVE COMPOENETS ASSUME 5\% ACCURACY } \\
\text { WITH 1/4 WATT POWER DISSIPATION AT 200 PPM/DEG.C (RESISTOR FAMILY) } \\
\end{array}$} \\
\hline 4 & \multicolumn{4}{|c|}{$\begin{array}{l}\text { THRESHOLD QUANTITIES ASSUME A BUYER/BROKER NEGOTIATION TOWARD A BREAK } \\
\text { AT THE NEXT PRICE LEVEL AS ACCOUNTED FOR IN THE RESULTS ABOVE. }\end{array}$} \\
\hline
\end{tabular}




\section{Appendix C-3 \\ Support Costs}

\section{SUPPORT COSTS}

\section{Chipset Evaluation Chip manufacturer REV Support}

The degree of success in chip manufacturer support will depend on

the sales volumne of the product and the direction of charger technology.

\section{Built Product Support}

\section{Note:}

Assume the Design Engineer is familiar with PLC technology and with the writing software for the chipset.

1: Startup Manufacturing Cost (PCB Board)

2: Box Build Assembly (Includes Tooling and Sourcing)

$\$ 3,000.00$

3: Field Installation and Support

$\$ 2,000.00$

4: Field Integration with the Utility Smart Meter

$\$ 2,000.00$

5: Post Instalation Support

$\$ 2,000.00$

Note Costs associated with construction permits, outside structures, bracing, and additional engineering are not included This is due to the focus of this study being placed on the physical integration og the charger communication. 